No. 04-9

\title{
Interstate Fiscal Disparity in State Fiscal Year 1999
}

\author{
Robert Tannenwald and Nicholas Turner
}

\begin{abstract}
:
This paper compares states in terms of their relative fiscal capacity, fiscal need, fiscal comfort, and tax effort in state fiscal year 1999 (FY1999). It is the most recent in a series initiated by the U.S. Advisory Commission on Intergovernmental Relations (ACIR) in 1962. As in previous studies, the authors use the representative tax system and representative expenditure system methodologies in their analysis. Compared with FY1997, the authors find less interstate disparity in fiscal capacity, fiscal need, and fiscal comfort. However, such disparity, though diminished, remains substantial. The New England and Mid-Atlantic regions remain the most "fiscally comfortable," while the East South Central and West South Central regions are still the most "fiscally stressed."
\end{abstract}

\section{JEL Classifications: H71, H72}

keywords: state and local government taxation; state and local government expenditure

errata: A number of errors that appeared in the original version of this paper have been corrected in this version. An itemized note detailing these changes appear on page 17.

Robert Tannenwald is an Assistant Vice President and Economist, and Nicholas Turner is a Policy Analyst at the Federal Reserve Bank of Boston.

Their e-mail addresses are: robert.tannenwald@bos.frb.org and nick.turner@bos.frb.org, respectively.

This paper, which may be revised, is available on the web site of the Federal Reserve Bank of Boston at http://www.bos.frb.org/economic/ppdp/index.htm.

The views expressed in this paper do not necessarily reflect those of the Federal Reserve Bank of Boston or the Federal Reserve System.

The authors wish to thank Lin Gong, Amanda Lydon, and Matthew Nagowski for their able research assistance and Borja Larrain for helpful suggestions on an earlier draft. Any errors, of course, are the authors' responsibility.

This version: April 2006 


\section{Introduction}

In state fiscal year 1999 (FY1999), the nation's state and local governments collectively enjoyed a surplus equal to 2.5 percent of their spending ${ }^{2}$. However, while fiscally healthy in the aggregate, states varied considerably in their fiscal condition. More importantly, the size of the nationwide state and local fiscal surplus provides no insight into differences across states in their inherent capacity to raise revenue, regardless of short-term economic fluctuations, nor into their exposure to long-run spending pressures that are difficult to control.

This paper attempts to compare states in terms of these two fiscal characteristics using data for FY1999. It is the fourth in a series of reports by Tannenwald, the last of which used data for FY1997. This series, in turn, succeeds a series of similar reports undertaken by the U.S. Advisory Commission on Intergovernmental Relations (ACIR) over a 30-year span from FY1962 to FY1991. Both sets of reports employ the representative tax system (RTS) and representative expenditure system (RES) approaches to measure these interstate differences.

The authors assume that the reader already possesses some familiarity with these approaches. Nevertheless, the basic concepts and strategies underlying them are briefly reviewed below, and detailed explanations are provided in the methodological appendix. Further discussions of key methodological issues can be found in Tannenwald (2001, 1999, and 1997) and ACIR (1995).

\section{Basic Concepts and Strategies}

Spending pressures and the capacity to raise revenues vary considerably from state to state. States facing circumstances that compel them to spend more per capita on a particular public service or to provide a wider array of services than the average state

\footnotetext{
${ }^{1}$ The state fiscal year runs from July through June, except in Alabama, Michigan, and the District of Columbia, where it ends in September; and in New York and Texas, where the fiscal year ends in March and August, respectively.

2 U.S. Census data on state and local government finances indicate that in FY 1999 state and local government “General Revenues" exceeded “Direct General Expenditures" by 2.5 percent.
} 
have high fiscal need. For example, other things being equal, a state with a large percentage of its population between the ages of 5 and 18 has a high need for spending on primary and secondary education. On the revenue side, a state with large economic flows and ample stocks of wealth traditionally taxed by state and local governments enjoys a high fiscal capacity. For example, other things being equal, a state rich in natural resources has a high potential to raise severance tax revenues. A state's fiscal comfort is its fiscal capacity relative to its fiscal need.

Differences across states in fiscal comfort reveal the degree of fiscal disparity within the nation. This measure is especially interesting to national policymakers, since a widely embraced goal of many nations possessing a federalist form of government is to narrow interstate or inter-provincial fiscal disparity. Concern about fiscal disparity rests primarily on two interrelated normative considerations. First, access to some minimum level of state and local public services is desirable (referred to in public finance terminology as a "merit good" argument). ${ }^{3}$ Second, as long as fiscal disparity exists, citizens in fiscally comfortable jurisdictions must bear a lower tax burden to obtain this minimum than citizens in fiscally stressed jurisdictions. Many analysts consider this situation to be inherently unfair. Moreover, it exacerbates fiscal disparity over the long run by trapping fiscally stressed states in a vicious circle. The more intensively they tax, the more they drive away capital and labor, the more fiscally stressed they become, the more they must raise tax burdens, and so on.

Tax effort, defined as revenues collected relative to capacity, offers insights into the degree to which a state utilizes its revenue-raising potential. Some policymakers view a state's overall tax effort relative to those of other states as an indicator of whether the burden of the tax system is "too high" - either because it is uncompetitive or because high tax effort implies that the state's state and local public sector is "too large." One should be cautious in drawing such conclusions, however, because a state might have to impose a high tax effort in order to raise a given level of public services if it is fiscally stressed. Moreover, simple indicators such as tax effort are not necessarily valid 
indicators of competitiveness (Tannenwald 1996, 2004). Conversely, other policymakers see a relatively low tax effort as an indication that a state's tax system is competitive and/or that it is not providing needed public services. Policymakers in states with low tax effort should be equally cautious in drawing such conclusions. If a state imposes a high tax effort on certain tax bases but not on others, policymakers sometimes conclude that the state's tax mix needs to be altered in ways that narrow differences in effort across taxes. Nevertheless, a state may have valid reasons for taxing some bases more intensively than others, for example, on grounds of equity, exportability, or administrative simplicity.

RTS assesses the relative ability of a state to raise revenue from a particular tax by levying a "standard" tax rate on a "standard" tax base. This tax rate is computed in the following manner: First, it is assumed that the nation as a whole imposes the tax on a uniformly defined base. This base equals the nationwide value of all economic stocks or flows that would be taxed if the base were defined comprehensively. If defined in this manner, the tax base would be devoid (to the extent feasible) of exemptions, exclusions, deductions, and other tax preferences that favor certain forms of economic activity over others or that provide tax relief to taxpayers in certain circumstances. For example, the comprehensively defined general sales tax base includes most services, even though most state and local governments levying a sales tax choose to exclude many services from their tax base. This broadly defined potential tax base is then divided into actual revenues collected from the tax in question from all state and local governments nationwide. The resulting ratio is the tax's standard rate. In effect, this tax rate is the nationwide tax effort for the tax. Table 1 presents the nationwide comprehensively defined base and standard tax rate in FY1999 for each of the commonly imposed state and local taxes.

The value of the standard base of each of these taxes is apportioned among the states and the District of Columbia. The state-specific tax base is then multiplied by the applicable standard tax rate and divided by the state's population to arrive at the state's

\footnotetext{
${ }^{3}$ See Musgrave (1984, pp. 78-79) for further discussion of the merit goods concept.
} 
per capita capacity to raise revenues from the tax. The per capita capacities of all the taxes are summed for each state to arrive at the state's total tax capacity. The states' tax capacities are then indexed to their nationwide counterpart to arrive at each state's RTS index value.

Analogous to the RTS approach to estimating fiscal capacity, the RES approach estimates the amount that state and local governments must spend to provide a standard level of service for each representative bundle of state and local spending. Six functions are influenced heavily by factors other than population: elementary and secondary education, higher education, public welfare, health and hospitals, highways, and police and corrections. In FY1999, these six functions accounted for about 70 percent of all state and local governmental expenditures. The need for spending on other functions, such as general administration, environmental protection, and housing, is assumed to be proportional to population.

For each of these six functions, the analyst implementing the RTS approach estimates a "workload" factor, that is, a determinant or a weighted index of determinants of the cost of providing a given level of service-other than the prices of inputs. For example, one determinant of the workload factor for highway expenditures is the number of vehicle-miles traveled, an indicator of maintenance and repair costs attributable to wear and tear from vehicular traffic. The other determinant, lane miles of streets and roads, reflects the sheer amount of pavement, signage, traffic lights, street lamps, and other road accessories that must be maintained and repaired. As an illustration of how to compute a state's workload-factor for highway expenditures, consider the case of Massachusetts. In FY1999, the Commonwealth accounted for 0.91 percent of the nation's lane miles of roadway and 1.93 percent of vehicle-miles traveled. The number of vehicle-miles traveled is weighted 4.71 (or .825/.175) times as heavily as the total number of lane miles in the workload factor for highways. ${ }^{4}$ Consequently,

\footnotetext{
${ }^{4}$ This weighting reflects analyses by engineers indicating that the intensity of roadway usage, and not the total length of roadway, is the primary determinant of the need for road and bridge maintenance and repair.
} 
Massachusetts' workload factor for highways was $0.175 \times 0.91+0.825 \times 1.93$, or 1.75 percent.

The nationwide spending by state and local governments on each function is then multiplied by the state's workload measure for that function to determine how much the state would have spent if it had provided a standard level of services, that is, if it had spent an average amount per "workload measure unit." For example, in FY1999 the nation's state and local governments spent $\$ 93.02$ billion on highways. With a workload measure of 1.75 percent, Massachusetts' spending on highways would have been approximately $\$ 1.63$ billion (.0175 X $\$ 93.02$ billion), or about $\$ 271$ per capita. Nationwide, per capita state and local spending on highways was \$341. Thus, Massachusetts' highway workload measure was lower than its share of the nation's population.

The next step in estimating a state's fiscal need index is to adjust its estimated per capita "standard" spending on each function for its relative cost of inputs. The complicated methodology for this adjustment is explained in Rafuse (1990a) and in this study's methodological appendix. In FY1999, Massachusetts' input costs for highway services were 2.1 percent higher than the national average. Consequently, its unadjusted per capita spending on highways was raised to $1.021 X \$ 271$, or $\$ 277,81.2$ percent of the national average.

For each state, the per capita standard spending levels on each function are totaled to obtain the state's per capita spending on the standard expenditure package. These totals are indexed to actual national per capita spending by state and local governments to arrive at the index value of fiscal need for each state. ${ }^{5}$

\footnotetext{
${ }^{5}$ The RES expenditure index is a crude measure of fiscal need. It fails to take into account many relevant factors. Severity of winter is a case in point. Other things being equal, a state with relatively severe winters will have a higher need for expenditures on roads and bridges because the cold, snow, and ice cause frost heaves and other structural damage. Winter severity also has
} 


\section{Estimated RTS Indexes for FY1999}

Table 2 presents indexes of fiscal capacity for the 50 states and the District of Columbia for several years from FY1987 through FY1999. In FY1999, as in the earlier years, states with relatively high capacities tend to be those with high average household incomes, high average property values, large tourist industries (for example, Hawaii and Nevada), or large stocks of energy resources. In New England, Connecticut, Massachusetts, and New Hampshire all ranked in the top quartile, and generally have done so since FY1987, thanks to high per capita incomes. High property values in all three states have also boosted their tax capacity in recent years. By contrast, Maine, Rhode Island, and Vermont, with lower average incomes and average property values, have tended to possess average or low fiscal capacities.

Chart 1 compares the fiscal capacities of the nine U.S. Census regions in FY1999 and FY1997. In both years, the New England, Mid-Atlantic, Pacific, and Mountain regions had above-average fiscal capacity, while the South Atlantic, East North Central, West North Central, East South Central, and West South Central regions had belowaverage capacity. As in interstate comparisons, the regions with high capacities tend to have high average incomes, and vice versa.

As Tannenwald has cautioned before (1999, 2001), drawing conclusions from inter-temporal comparisons of fiscal capacities is fraught with danger, primarily because the author has modified his methodology from year to year. However, comparisons between FY1999 and FY1997 are somewhat less problematic because the methodologies employed in both years are almost identical. The dispersion among the states and the District of Columbia narrowed between the two years, indicated by a decline in the interstate standard deviation from 19.3 to 13.3. Changes in measures of skewness and kurtosis indicate that in FY1999 the distribution of fiscal capacities was also more symmetrical and closer to normal than in FY1997. These changes in variation were attributable in part to sharp declines in the RTS index of certain energy-producing states

an impact on the need for welfare spending. A homeless person in Maine is in danger of freezing to death in the winter. Not so in Florida. 
that generally register high RTS values, such as Alaska, Wyoming, and Colorado. These declines, in turn, resulted from falling energy prices. A sharp decline in Hawaii's fiscal capacity index, attributable to deterioration in the state's tourist industry, also contributed to the narrowing in dispersion.

However, the decline in California's RTS index value from 116 to 111, which also helped to narrow dispersion, may reflect measurement error rather than an actual fall in the state's relative tax capacity. The decline in California's RTS index is largely attributable to a reduction in its property tax capacity, whose RTS index fell between FY1997 and FY1999 from 145 to 135 . Because of Proposition 13 and a lack of available data on the aggregate fair market value of the state's entire stock of real estate, California's property tax capacity is more difficult to estimate than that of any other state.

Proposition 13 limits the annual increase in the assessed value of any taxable property to one percent a year, unless ownership of the property changes hands, in which case the property is reassessed at its purchase price. Since only a fraction of all property in California is sold in any given year, the aggregate assessed value of taxable property in California is far below its fair market value. While California's Department of Revenue estimates total assessed value each year, no governmental agency in California estimates fair market value. Consequently, in order to estimate California's standard property tax base, the authors employed the following crude method for both FY1997 and FY1999: First, they assumed that the statewide ratio of assessed-to-fairmarket value was the same as that estimated for Los Angeles County in FY1996 by Sheffrin and Sexton (1998). Second, they divided the aggregate property tax base into several components and estimated the value of each component from data for the latest year available. Third, they extrapolated these estimates to FY1997 and FY1999 using a variety of data sources on the value of new construction and changes in real estate prices. (Details are provided in the methodological appendix.) Fourth, they summed the extrapolated values across all property components to arrive at the estimated value of the state's potential property tax base. Finally, they compared this "extrapolated" 
estimate with the one based on the ratio estimated by Sheffrin and Sexton and chose the larger of the two, because historically the extrapolation technique seemed to be producing estimates that were too low. In FY1997, the estimate based on the Sheffrin and Sexton ratio was larger, while in FY1999 the "extrapolated" estimate was larger. Consequently, the FY1999 estimate of California's property tax capacity was derived from a different methodology than the FY1997 estimate. Had the FY1997 estimate been based on the extrapolation technique, it would have been lower, California's total tax capacity index for FY1997 would have been lower, and this index would not have fallen as much as it did between the two years.

For FY1999, both methodologies are especially problematic. The extrapolation technique requires the analyst to project residential property values out from the previous decennial Census of Housing. For FY1999 estimates, it was therefore necessary to extrapolate data out from the 1990 Census. As for the alternative technique, it is not at all clear that Sheffrin and Sexton's estimated ratios of assessed-to-fair-market value for FY1996 were as accurate three years later, in FY1999, as they were one year later, in FY1997. Furthermore, the average ratio of assessed-to-fair-market value in Los Angeles County may not be representative of the comparable ratio in the rest of the state.

Because of the difficulty of estimating California's property tax capacity and, therefore, total tax capacity, one should be especially cautious in interpreting the decline in the average RTS index of the Pacific Region between FY1997 and FY1999, as shown in Chart 1. True, some of the decline reflects declining capacity in Hawaii and Alaska. However, in FY1999, 76.5 percent of the region's state and local tax revenues were collected by California. Consequently, the region's estimated decline is heavily influenced by the RTS estimates for that state.

While fiscal capacity in New England remained constant from 1997 to 1999, the RTS rankings of several states in the region changed. Maine's rank fell from $27^{\text {th }}$ to $36^{\text {th }}$, although its index declined by only three points. This drop reflects declines across most tax categories except the sales tax, individual income tax, and property tax. Slow growth in the capacity of these taxes - a one-point increase in the index of both sales tax 
and individual income tax capacity and just over a four-point increase in the property tax capacity index - could not stop the overall tax capacity index from eroding slightly. New Hampshire's state rank climbed five places, the largest gain among the New England States, largely because its index of corporate income tax capacity increased by 10 points. The state's property tax capacity index also rose slightly, up one point, driving the overall tax capacity index up four points. Massachusetts' rank increased three places, from $11^{\text {th }}$ to $8^{\text {th }}$. A three-point increase in the general sales tax capacity index and a one- point increase in the personal income tax capacity index helped the overall capacity index climb by two points. An eight-point drop in the property tax capacity index combined with a one-point drop in the sales tax capacity index caused Vermont's ranking to slip three places. However, the state's overall tax capacity index was off by only two points.

\section{Results of RES Index for FY1999}

Results for state fiscal need indexes and rankings appear in Table 3 and Chart 2. As in past years, the dispersion in fiscal need was considerably narrower than the dispersion in fiscal capacity. ${ }^{6}$ With the exception of Connecticut, the New England states displayed their characteristically low fiscal need, all ranking in the bottom quartile. Connecticut's RES has been by far the highest in the region since FY1991, mostly because of its high unit labor costs (in FY1999, second highest in the nation behind New Jersey and 17 percent above the national average). States with high fiscal need in all regions tend to have high poverty rates, high concentrations of population in the 5-17 age bracket, high crime rates, or some combination of the three. They tend to be concentrated in the South and Southwest (for example, in New Mexico, Texas, Louisiana, Mississippi, Georgia, Arizona, and Alabama). California and the District of Columbia also suffer from chronically high fiscal need.

${ }^{6}$ The standard deviation of the RES index was 5.5 compared with a standard deviation of 13.3 for the RTS index. 
Between FY1997 and FY1999, the dispersion across states in fiscal need narrowed, as did the dispersion in fiscal capacity. Two demographic factors are primarily responsible for changes in a state's fiscal need index between these two years. One is a change in the state's relative poverty rate, an important determinant of need for public welfare and health and hospital expenditures. The other is a change in the relative importance of school-age children in the state's population mix, the major determinant of need for spending on primary and secondary education.

Of the five states with the largest increases in their fiscal need index, twoOregon and Nevada-experienced significant increases in both of these factors. The increases in fiscal need of the other three states-New Hampshire, North Carolina, and North Dakota - were driven mostly by changes in relative poverty levels. Similarly, in the states with relatively large declines in their fiscal need index-such as Connecticut, Florida, Kentucky, South Dakota, and Tennessee-the primary drivers were declines in poverty levels and/or rises in the relative importance of 5-to-17-year-olds in the population mix.

\section{The Correlation between Fiscal Capacity and Fiscal Need}

Tannenwald (1998) asserts that this correlation has repercussions for the "devolution" debate. In the context of intergovernmental relations, devolution means the "devolving" of fiscal responsibilities from higher to lower levels of government, especially from the federal to the state or provincial level. Opponents of devolution in the United States are concerned that states with high fiscal need and low fiscal capacity would be unable to provide their residents with an acceptable level of state and local public services. Opponents would be less concerned if states with the highest fiscal need also had the highest fiscal capacity. The upper right quadrant of Figure 1 shows that only seven states have higher-than-average need and capacity. In addition, only 13 states are in the upper left quadrant - the "best off" region - where states have both low fiscal need and high fiscal capacity. The remaining states reside in the lower quadrants, 
or on the border of these two regions. Several southern and western states reside in the "worst off" region, facing high fiscal need while having low fiscal capacity.

Nationally, the correlation coefficient between fiscal need and fiscal capacity was statistically insignificant in FY1999 (-0.023) when all 50 states and D.C. are included as observations. When D.C. , an outlier, is dropped, the coefficient, though more negative $(-0.203)$, remains statistically significant.

\section{Results of Fiscal Comfort in FY1999}

A measure of fiscal comfort for each state was constructed by dividing the state tax capacity index by its index of fiscal need. Results for the Census regions appear in Chart 3 and for the individual states in Table 4. As in FY1997, New England was the most fiscally comfortable region despite a three-percentage-point decline in its fiscal comfort index. Connecticut, Massachusetts, and New Hampshire were among the top six states in fiscal comfort. Within the region, only Rhode Island (34th) was in the second half of the rankings.

The fiscal comfort of the Census regions converged between the two years. The Mid-Atlantic, South Atlantic, and East South Central regions all became more "fiscally comfortable," while the East North Central, West North Central, Mountain, and Pacific regions all became more "fiscally stressed." Disparity across the states in fiscal comfort indexes also diminished, although not always for the same reasons. Since fiscal comfort is a composite of two indexes, movement in one or the other, or both, can drive the changes. Alaska's fiscal comfort rank dropped from $7^{\text {th }}$ to $26^{\text {th }}$, in large part because of its declining severance tax and overall fiscal capacity. By contrast, Florida's decline in fiscal need raised its fiscal comfort rank from $23^{\text {rd }}$ to $9^{\text {th }}$.

While it is tempting to reach back to earlier years on Table 4 and compare fiscal comfort indexes, it is important to keep in mind that methodologies have not been

constant across years. Even between 1997 and 1999, when the methodologies were very similar, subtle changes in data sources could be driving the observed results. Therefore, 
it is important to interpret results across years, especially prior to 1997, with great caution.

\section{Results of Tax Effort in FY1999}

In FY1999, the Mid-Atlantic states had the highest average tax effort-126. New England's average index of 108 and the East North Central's 102 also exceed 100. The remaining Census regions all register lower-than-average tax effort indexes, ranging from the West North Central's 99 to the East South Central's 87. (See Chart 4.) As in FY1997, the District of Columbia and New York had the highest tax efforts by far. Within New England, three states (Connecticut, Rhode Island, and Maine) ranked among the top five, while Massachusetts and Vermont ranked among the top 15. New Hampshire, by contrast, had the lowest tax effort of any state in the nation except Nevada.

States registering the largest absolute changes in their tax effort index between FY1997 and FY1999 (for example, California, Colorado, Iowa, Louisiana, Nebraska, Washington, West Virginia, Minnesota, and Wisconsin) tended to be those with the largest absolute changes in their index of property tax effort. (See Table 5.) ${ }^{7}$ Changes in the index of property tax effort were, in turn, negatively correlated with changes in the index of property tax capacity. ${ }^{8}$ The precipitous decline in Alaska's tax effort index (from 110 to 87 ) was surprising, given the state's steep decline in severance tax capacity. However, severance tax collections fell nearly three times more than severance tax capacity in the state. A further analysis of the state's severance tax rate structure might reveal why severance tax revenues are so elastic with respect to the tax's base.

Within New England, Maine registered the largest percentage-point increase in its tax effort index, from 112 to 118. Rising effort in taxing personal income, corporate

\footnotetext{
7 The correlation coefficient between total tax effort index and property tax effort index was 0.51 , significant at the 0.01 level. Keep in mind, however, potential data problems exist in estimating California's relative property tax capacity and, therefore, relative property tax effort.

8 The correlation coefficient between the property tax effort index and the property tax capacity index was -0.24 , significant at the 0.10 level.
} 
income, and, to a lesser extent, retail sales fueled the overall tax effort increase. In contrast, Maine's property tax effort actually declined. Moreover, it did so despite a sharp drop in the state's property tax capacity. Perhaps these anomalous trends reflect Mainers' growing concern about the ratio of property tax collections in their state to their personal income-the second highest property tax-to-income ratio in the nation in FY1999. Massachusetts lowered its tax effort index by three points primarily because it cut income taxes substantially. These cuts outweighed an increase in property tax effort, in turn due in part to a decline of four points in the index of property tax capacity.

\section{State Preferences for Public Services}

Tannenwald (1998) notes that variations in preferences for public services may be inferred from the correlation between state tax effort and fiscal comfort. If all states desired a uniform level of services, then fiscally uncomfortable states would have to tax their bases relatively intensely or exhibit a high tax effort to provide the desired level of services. In contrast, a state with high fiscal comfort could have a relatively low tax effort and still provide the desired level of services. In other words, the absence of a negative correlation between tax effort and fiscal comfort suggests that states with low levels of public expenditures tend to spend less because they want to, not because they are constrained by a lack of revenue.

Figure 2 shows the relationship between tax effort and fiscal comfort indexes for the 50 states and the District of Columbia. Only five states reside in the region of high tax effort and low fiscal comfort (upper left hand quadrant) while many states reside in the low tax effort, low fiscal comfort region (lower left) or the high tax effort, high fiscal comfort region (upper right). This distribution is exactly the opposite of what one would expect to find if preferences for public goods were homogenous across states. However, there are 14 states that reside in the high fiscal comfort, low tax effort region (lower right). The correlation between fiscal comfort and tax effort for all 50 states and D.C. was weakly positive at 0.047. If D.C. is removed, the correlation is even weaker at 0.0081. Neither correlation achieves statistical significance, suggesting that there is no 
observed relationship between fiscal comfort and tax effort. This fact seems to bolster the argument that states have unique preferences for public services and that these preferences are not necessarily constrained by state tax capacity.

\section{Conclusion}

The authors' conclusions from FY1999 data are the same as those drawn from FY1997 data. Fiscal disparity, although diminished, is still substantial. The New England and Mid-Atlantic states remain among the most "fiscally comfortable" while those in the East South Central and West South Central are the most fiscally stressed. Differences in tax effort also persist. The lack of a correlation between tax effort and fiscal comfort implies that states also differ significantly in their preference for public service levels. Other things being equal, fiscally comfortable states have a stronger preference than fiscally stressed states for public services. If they did not, tax effort and fiscal comfort would be negatively correlated. Consequently, fiscally equalizing aid could induce fiscally stressed states to provide an inefficiently high level of public services, and vice versa.

Nevertheless, the persistence of fiscal disparity suggests that perhaps Congress should increase the degree of fiscally equalizing intergovernmental aid..$^{9}$ The argument in favor of such a policy grounded on equity and the merit good concept still holds. Other things being equal, households in similar economic circumstances should bear similar tax burdens in order to obtain a socially desirable minimum level of state and local public services. Moreover, modifying formulas that allocate federal aid more in favor of fiscally stressed states could ameliorate some of the disadvantages they sometimes face in economic competition for workers and employers.

\footnotetext{
${ }_{9}^{9}$ Several existing federal aid programs, such as Medicaid and the State Children's Health Insurance Program, are fiscally equalizing. The measures of state fiscal capacity used in their allocation formulas are based on state per capita personal income.
} 


\section{References}

Musgrave, Richard. and Peggy Musgrave. 1984. Public Finance in Theory and Practice. New York, NY: McGraw Hill.

Rafuse, Robert. 1990a. "Representative Expenditures: Addressing the Neglected Dimension of Fiscal Capacity." Washington, DC: U.S. Advisory Commission on Intergovernmental Relations.

- 1990b. "A Walk on the Expenditure Side: 'Needs' and Fiscal Capacity." Intergovernmental Perspective, U.S. Advisory Commission on Intergovernmental Relations magazine. Fall: 25-30.

Sheffrin, Steven M. and Terri Sexton. 1998. Proposition 13 in Recession and Recovery. San Francisco: Public Policy Institute of California.

Surrey, Stanley S. 1973. Pathways to Tax Reform: The Concept of Tax Expenditures. Cambridge, MA: Harvard University Press.

Tannenwald, Robert. 1996. "State Business Tax Climate: How Should It Be Measured and How Important Is It?" New England Economic Review January/February.

. 1998. "Come the Devolution, Will States Be Able to Respond?" New

England Economic Review May/June: 58-83.

. 1999. "Fiscal Disparity Among the States Revisited." New England Economic Review July/August: 3-25.

. 2002. "Interstate Fiscal Disparity in 1997." New England Economic Review Third Quarter: 17-33.

. 2004. “Massachusetts Business Taxes: Unfair? Inadequate? Uncompetitive?" Federal Reserve Bank of Boston, Public Policy Discussion Papers, paper no. 04-4.

U.S. Advisory Commission on Intergovernmental Relations. 1993. RTS State Revenue Capacity and Effort-1991. Washington, DC: U.S. Government Printing Office. 


\section{Errata-April 2006}

Multiple errors have been found in the December 2004 version of this paper; they have been corrected as of April 2006.

Due to a rounding error in applying the representative rate to each state's property tax base, the original property tax calculations (as presented in the Appendix Table on page 69) were off by 2.5 percent. Because the indices used in this paper are rounded to the nearest whole number, this error was not large enough to affect every state's property tax effort index -45 states experienced an upward revision on their property tax effort index of one point.

This error also had repercussions for the overall indices of tax capacity, fiscal capacity, and tax effort as originally reported. 12 states experienced a change in one or more of these measures as a result of our error-all of one point. In turn, four Census regions also encountered a change in either their tax capacity or tax effort indices, or both.

All of the figures, charts, and tables have been updated to reflect these changes, and all references to erroneous statistics in the text have been corrected.

Finally, two typos were discovered on Table 1 of the paper. The standard revenue bases were incorrectly reported for both the 'general sales and gross receipts' category as well as the 'other taxes' category.

In all instances, these errors were not nearly large enough to impact any of this paper's findings or discussion. Even so, the authors regret their errors and assume full responsibility for them. 
Figure 1: Correlations between Fiscal Capacity and Fiscal Need

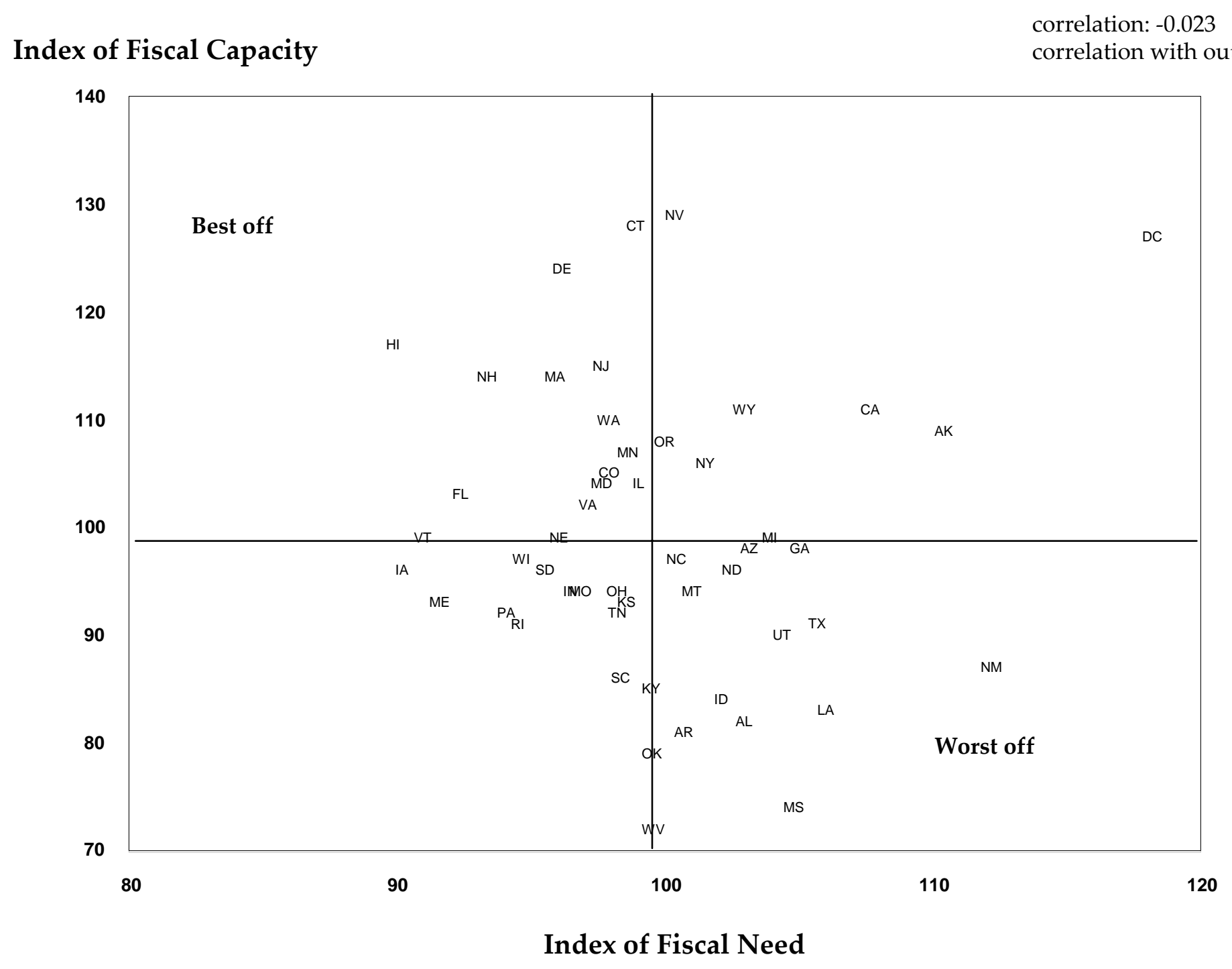


Figure 2: Correlations between Tax Effort and Fiscal Comfort

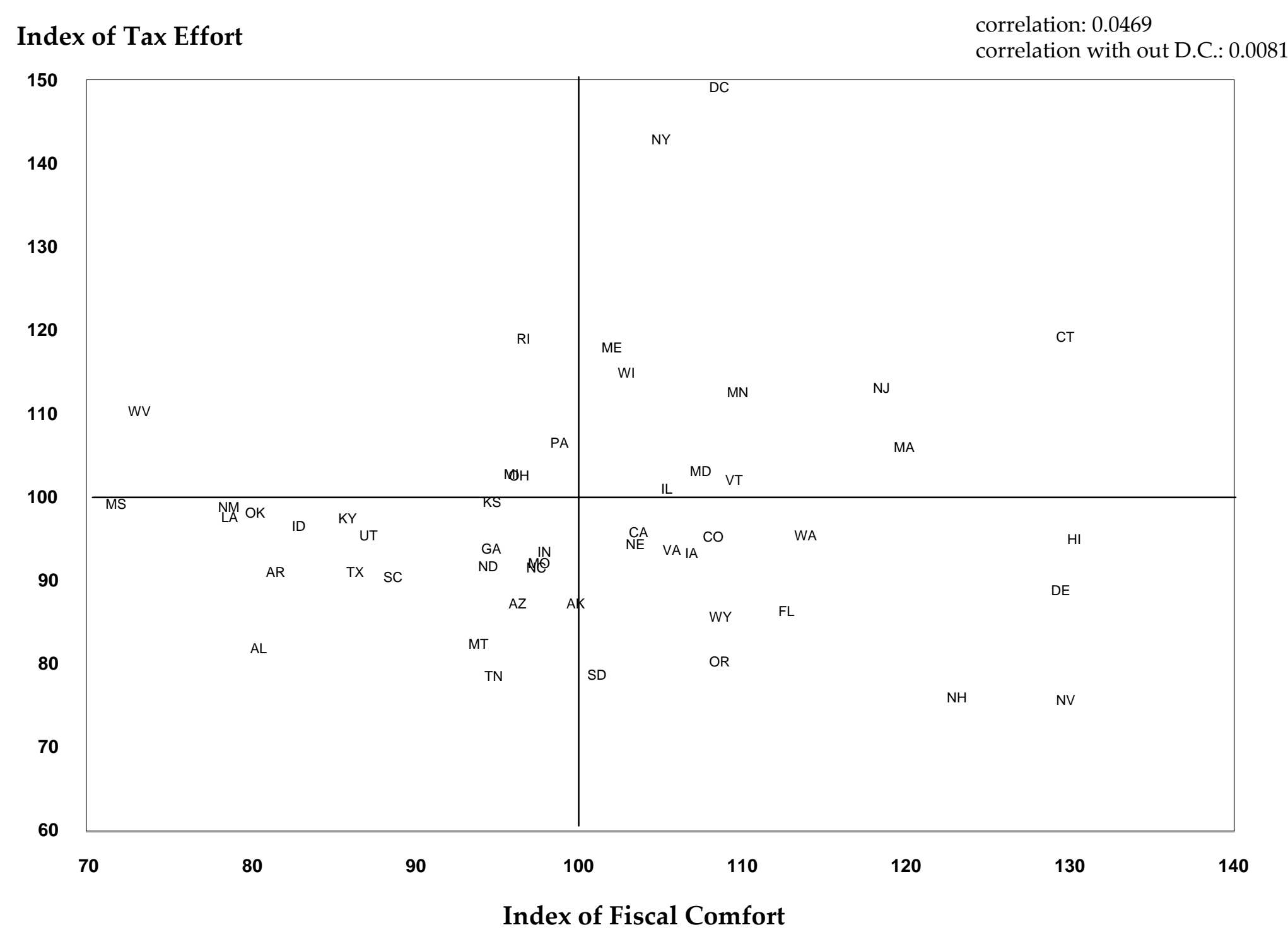


Chart 1

Index of Fiscal Capacity by Region

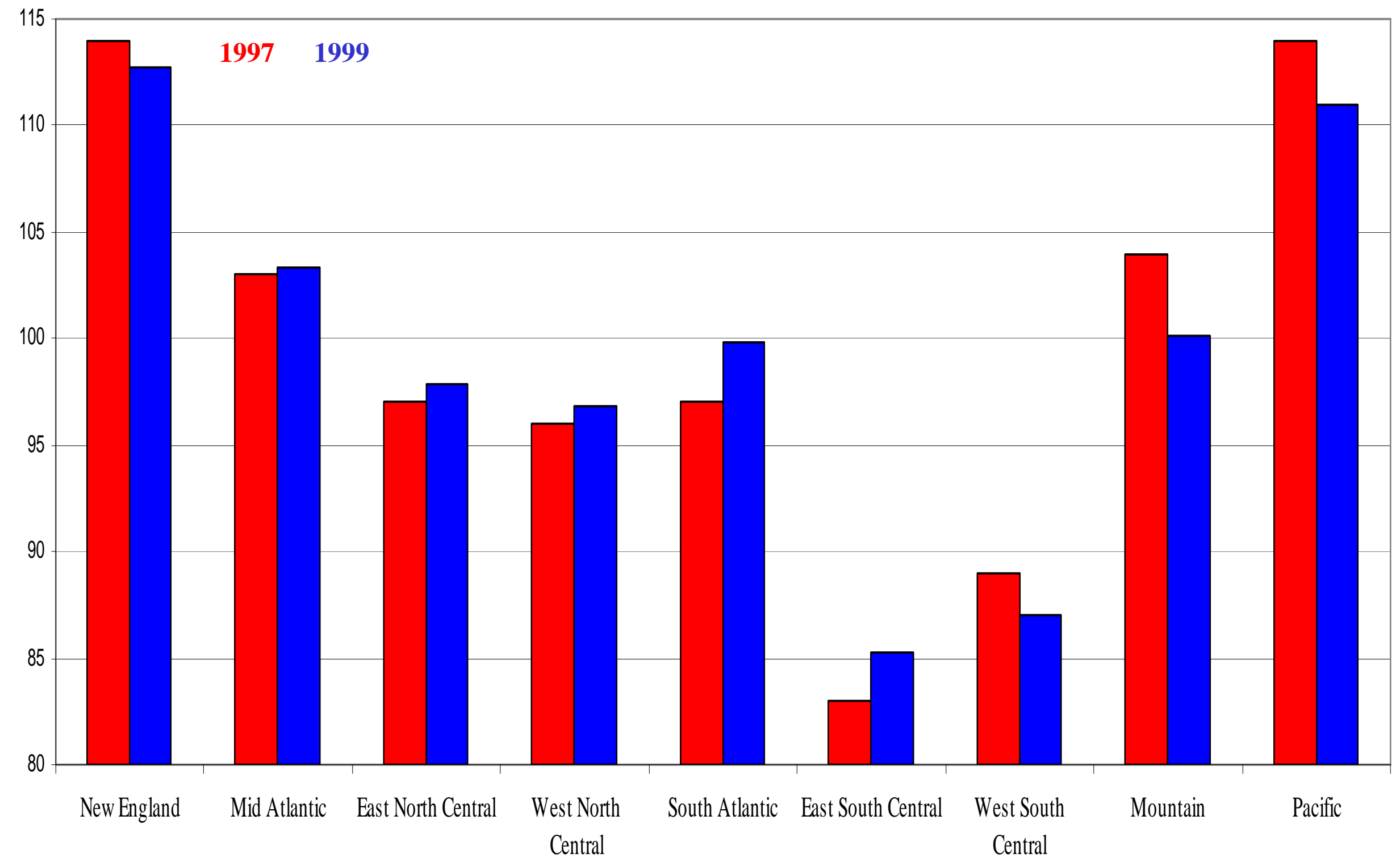


Chart 2

Index of Fiscal Need By Region

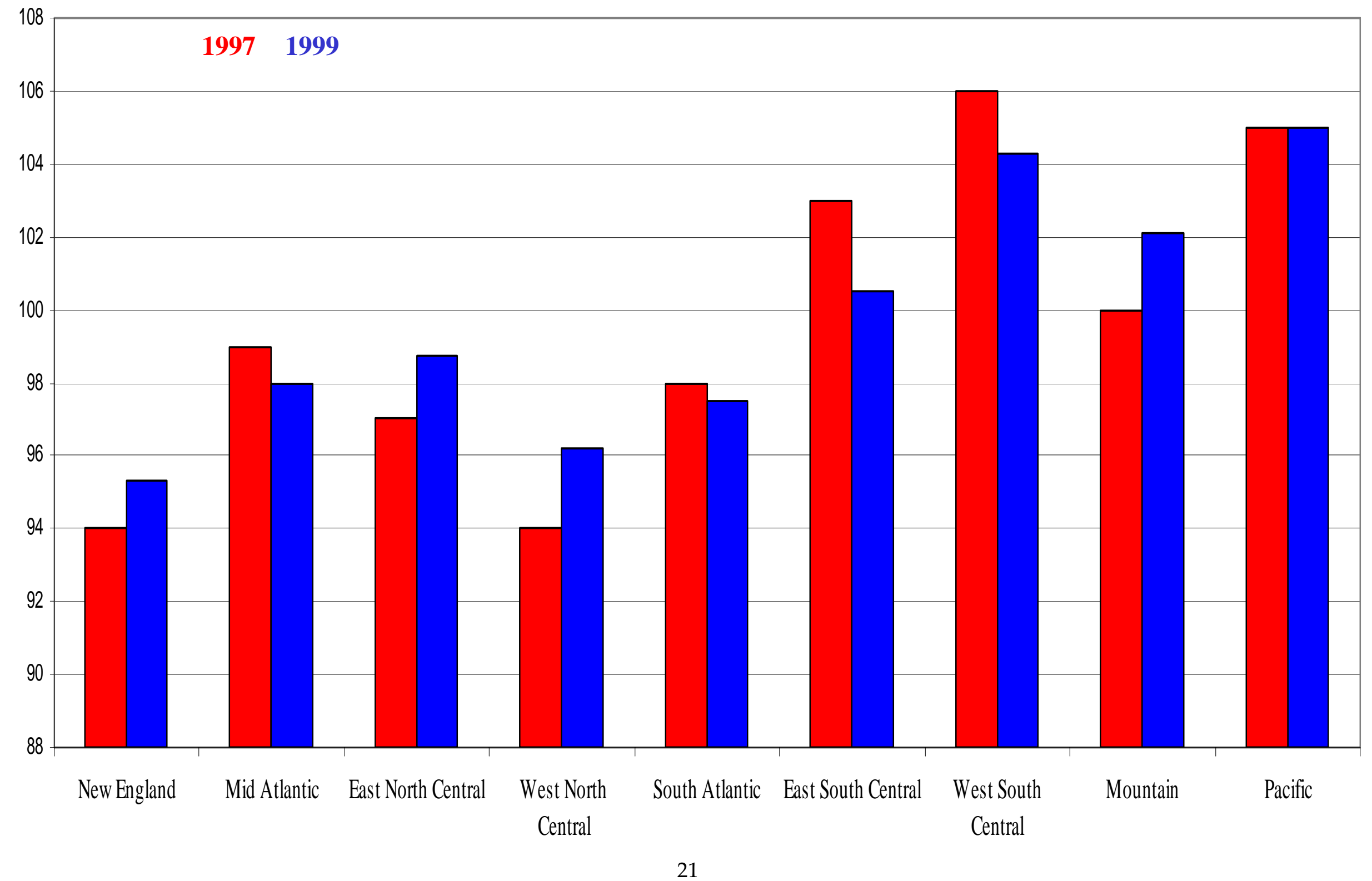


Chart 3

Index of Fiscal Comfort by Region

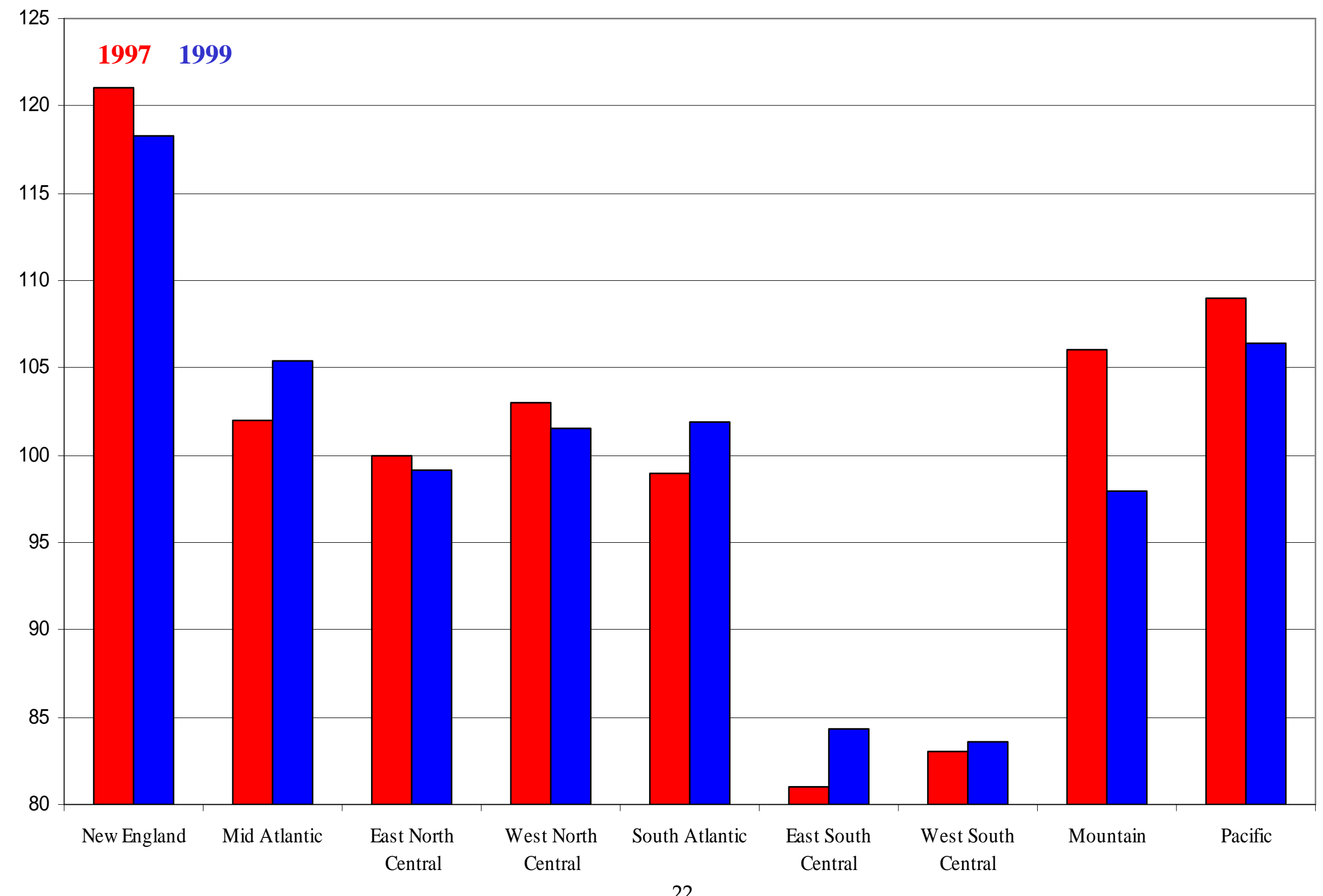




\section{Chart 4}

Index of Tax Effort by Region

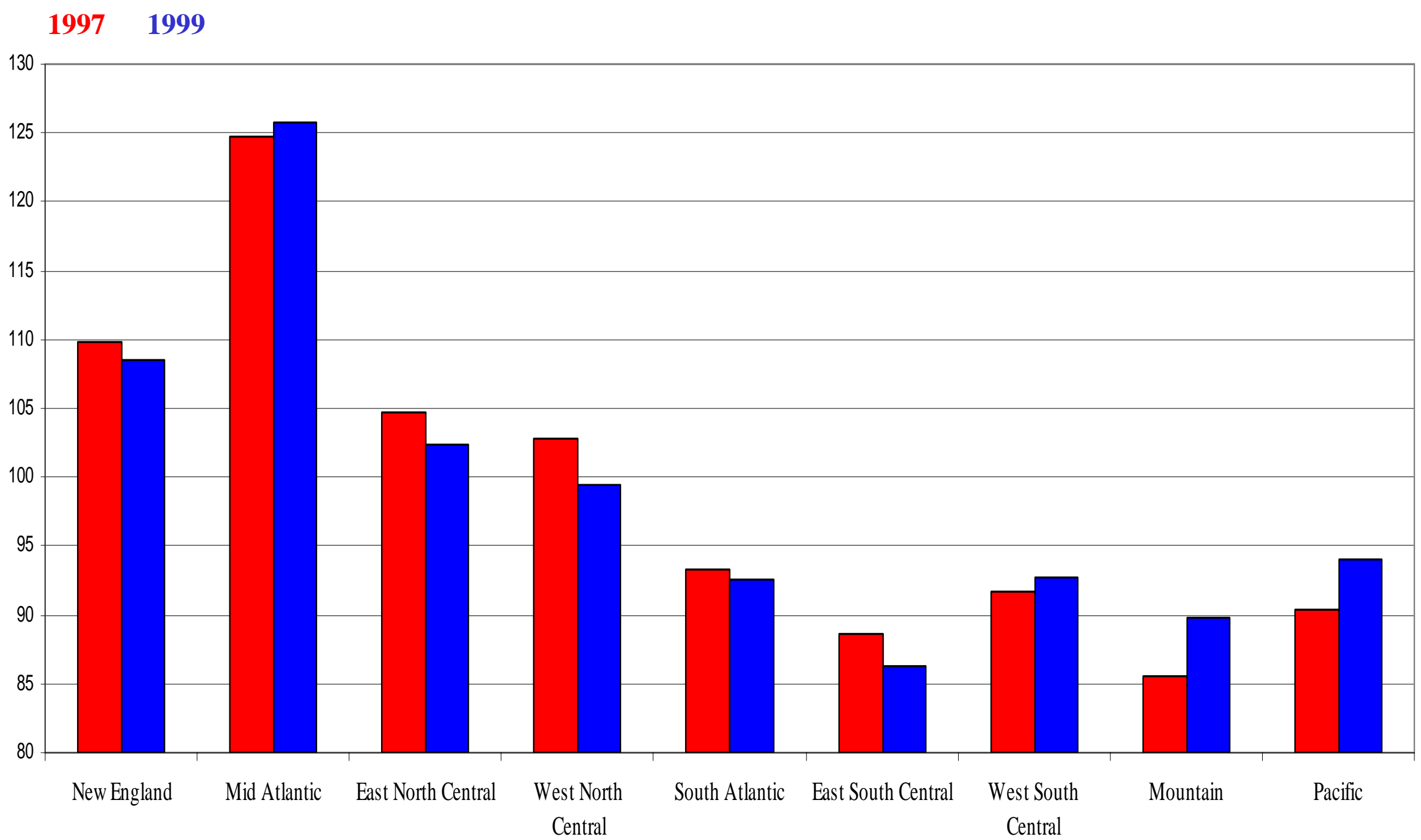


Table 1: Design of Representative Tax System for Fiscal Year 1999

State-Local Tax Collections

Standard Revenue Bases and Tax Rates

\begin{tabular}{|c|c|c|c|c|}
\hline Revenue Source & $\begin{array}{c}\text { Amount } \\
\text { (\$ billions) }\end{array}$ & $\begin{array}{c}\text { Percent of } \\
\text { Total }\end{array}$ & $\begin{array}{l}\text { Base* }^{*} \\
\text { (billions) }\end{array}$ & Rate \\
\hline General Sales and Gross Reciepts & 200.63 & 24.48 & $2,981.49$ & 6.73 cents per dollar \\
\hline Selective Sales & 77.30 & 9.43 & $1,546.83$ & \\
\hline Motor Fuel & 30.09 & 3.67 & 158.89 & 18.94 cents per gallon \\
\hline Alcoholic Beverages & 4.18 & 0.51 & 0.477 & $\$ 8.76 /$ gallon \\
\hline Distilled Spirits & 1.89 & 0.23 & 0.139 & \$13.65/gallon \\
\hline Beer & 1.85 & 0.23 & 0.271 & $\$ 6.81 /$ gallon \\
\hline Wine & 0.442 & 0.05 & 0.068 & \$6.54/gallon \\
\hline Tobacco & 8.37 & 1.02 & 21.96 & 38.13 cents per pack \\
\hline Insurance & 9.62 & 1.17 & 739.55 & 1.30 cents per dollar \\
\hline Public Utilities & 17.17 & 2.09 & 518.83 & 3.31 cents per dollar \\
\hline Pari-Mutuel & 0.38 & 0.05 & 18.16 & 2.10 cents per dollar \\
\hline Amusements & 3.29 & 0.40 & 88.96 & 3.70 cents per dollar \\
\hline License Taxes & 24.16 & 2.95 & & \\
\hline Motor Vehicles & 15.34 & 1.87 & 0.214 & \$71.65/license \\
\hline Vehicle Operator & 1.29 & 0.16 & 0.186 & \$6.94/license \\
\hline Corporation & 6.36 & 0.78 & 0.005 & \$1177.68/license \\
\hline Fishing and Hunting & 1.17 & 0.14 & 0.071 & \$16.45/license \\
\hline Personal Income Tax & 189.31 & 23.10 & $5,311.02$ & 3.35 cents per dollar \\
\hline Corporate Income Tax & 33.92 & 4.14 & 630.36 & 5.38 cents per dollar \\
\hline Property Taxes & 239.67 & 29.24 & $20,188.36$ & 1.19 cents per dollar \\
\hline Estate and Gift Tax & 7.52 & 0.92 & 28.39 & 26.49 cents per dollar \\
\hline Severance Tax & 3.14 & 0.38 & 127.85 & 2.45 cents per dollar \\
\hline Other Taxes & 43.96 & 5.36 & $7,599.01$ & 0.58 cents per dollar \\
\hline
\end{tabular}

$\begin{array}{lll}\text { RTS TOTAL } & 819.61 & 100.00\end{array}$

*Units shown in denominator of rate column

Numbers may not be exact due to rounding. 


\section{Table 2: Index of Fiscal Capacity}

National Average $=100$

\begin{tabular}{|c|c|c|c|c|c|c|c|c|c|c|c|c|}
\hline \multirow{2}{*}{$\underline{\text { New England States }}$} & \multicolumn{2}{|c|}{1999} & \multicolumn{2}{|c|}{1997} & \multicolumn{2}{|c|}{1996} & \multicolumn{2}{|c|}{1994} & \multicolumn{2}{|c|}{1991} & \multicolumn{2}{|c|}{1987} \\
\hline & $\begin{array}{c}\text { Index } \\
\text { (1) }\end{array}$ & $\begin{array}{c}\text { Rank } \\
\text { (2) }\end{array}$ & $\begin{array}{c}\text { Index } \\
\text { (3) }\end{array}$ & $\begin{array}{c}\text { Rank } \\
(4)\end{array}$ & $\begin{array}{c}\text { Index } \\
(5)\end{array}$ & $\begin{array}{c}\text { Rank } \\
(6)\end{array}$ & $\begin{array}{c}\text { Index } \\
(7)\end{array}$ & $\begin{array}{c}\text { Rank } \\
(8)\end{array}$ & $\begin{array}{c}\text { Index } \\
(9)\end{array}$ & $\begin{array}{c}\text { Rank } \\
(10)\end{array}$ & $\begin{array}{c}\text { Index } \\
\text { (11) }\end{array}$ & $\begin{array}{l}\text { Rank } \\
\text { (12) }\end{array}$ \\
\hline Connecticut & 127 & 2 & 129 & 3 & 129 & 2 & 132 & 3 & 130 & 4 & 139 & 2 \\
\hline Massachusetts & 114 & 8 & 112 & 11 & 116 & 9 & 114 & 9 & 117 & 9 & 127 & 4 \\
\hline New Hampshire & 114 & 7 & 110 & 12 & 118 & 8 & 107 & 12 & 110 & 11 & 123 & 6 \\
\hline Vermont & 99 & 22 & 101 & 19 & 99 & 21 & 95 & 29 & 105 & 15 & 103 & 17 \\
\hline Maine & 92 & 36 & 95 & 27 & 89 & 42 & 88 & 43 & 95 & 24 & 97 & 22 \\
\hline Rhode Island & 91 & 39 & 92 & 38 & 91 & 39 & 91 & 38 & 89 & 38 & 96 & 24 \\
\hline \multicolumn{13}{|l|}{ Other States } \\
\hline Nevada & 129 & 1 & 129 & 4 & 141 & 1 & 142 & 1 & 128 & 5 & 110 & 12 \\
\hline D.C. & 127 & 3 & 123 & 6 & 126 & 5 & 125 & 5 & 123 & 7 & 122 & 7 \\
\hline Delaware & 123 & 4 & 120 & 7 & 121 & 6 & 119 & 8 & 125 & 6 & 124 & 5 \\
\hline Hawaii & 116 & 5 & 130 & 2 & 120 & 7 & 125 & 5 & 146 & 2 & 113 & 10 \\
\hline New Jersey & 114 & 6 & 114 & 10 & 116 & 9 & 124 & 7 & 119 & 8 & 122 & 7 \\
\hline California & 111 & 9 & 116 & 8 & 103 & 17 & 105 & 14 & 115 & 10 & 117 & 9 \\
\hline Wyoming & 111 & 10 & 125 & 5 & 127 & 3 & 128 & 4 & 134 & 3 & 137 & 3 \\
\hline Washington & 110 & 11 & 101 & 18 & 104 & 16 & 102 & 18 & 108 & 13 & 99 & 20 \\
\hline Alaska & 109 & 12 & 133 & 1 & 127 & 3 & 135 & 2 & 178 & 1 & 169 & 1 \\
\hline Oregon & 108 & 13 & 103 & 15 & 103 & 17 & 99 & 21 & 100 & 21 & 92 & 29 \\
\hline Minnesota & 108 & 14 & 103 & 17 & 107 & 15 & 104 & 15 & 101 & 20 & 104 & 16 \\
\hline New York & 106 & 15 & 106 & 13 & 109 & 13 & 103 & 17 & 103 & 16 & 108 & 14 \\
\hline Colorado & 105 & 16 & 115 & 9 & 114 & 11 & 110 & 10 & 109 & 12 & 111 & 11 \\
\hline Illinois & 104 & 17 & 103 & 16 & 110 & 12 & 108 & 11 & 102 & 19 & 97 & 22 \\
\hline Maryland & 104 & 18 & 106 & 14 & 108 & 14 & 107 & 12 & 106 & 14 & 109 & 13 \\
\hline Florida & 103 & 19 & 98 & 22 & 100 & 20 & 100 & 20 & 103 & 16 & 105 & 15 \\
\hline Virginia & 102 & 20 & 101 & 20 & 101 & 19 & 104 & 15 & 103 & 16 & 102 & 18 \\
\hline Michigan & 99 & 21 & 96 & 26 & 98 & 24 & 101 & 19 & 94 & 26 & 95 & 25 \\
\hline Nebraska & 98 & 23 & 98 & 24 & 99 & 21 & 96 & 23 & 95 & 24 & 91 & 31 \\
\hline Arizona & 98 & 24 & 100 & 21 & 94 & 35 & 93 & 34 & 94 & 26 & 100 & 19 \\
\hline Georgia & 98 & 25 & 98 & 23 & 96 & 30 & 95 & 29 & 91 & 32 & 94 & 26 \\
\hline North Carolina & 97 & 26 & 93 & 35 & 92 & 36 & 92 & 36 & 93 & 28 & 90 & 34 \\
\hline Wisconsin & 96 & 27 & 93 & 34 & 97 & 25 & 96 & 23 & 90 & 36 & 88 & 36 \\
\hline North Dakota & 96 & 28 & 96 & 25 & 97 & 25 & 94 & 33 & 91 & 32 & 90 & 34 \\
\hline lowa & 96 & 29 & 94 & 29 & 97 & 25 & 93 & 34 & 93 & 28 & 84 & 41 \\
\hline South Dakota & 96 & 30 & 94 & 30 & 95 & 33 & 91 & 38 & 86 & 42 & 78 & 46 \\
\hline Indiana & 94 & 31 & 95 & 28 & 97 & 25 & 96 & 23 & 90 & 36 & 87 & 37 \\
\hline Montana & 94 & 32 & 92 & 36 & 99 & 21 & 96 & 23 & 91 & 32 & 87 & 37 \\
\hline Ohio & 94 & 33 & 94 & 31 & 96 & 30 & 97 & 22 & 93 & 28 & 91 & 31 \\
\hline Missouri & 93 & 34 & 93 & 33 & 97 & 25 & 95 & 29 & 91 & 32 & 91 & 32 \\
\hline Kansas & 92 & 35 & 94 & 32 & 96 & 30 & 96 & 23 & 93 & 28 & 93 & 27 \\
\hline Tennessee & 92 & 37 & 90 & 42 & 92 & 36 & 90 & 40 & 82 & 45 & 84 & 41 \\
\hline Pennsylvania & 92 & 38 & 92 & 39 & 95 & 33 & 96 & 23 & 96 & 23 & 92 & 29 \\
\hline Texas & 90 & 40 & 91 & 40 & 91 & 39 & 95 & 29 & 97 & 22 & 99 & 20 \\
\hline Utah & 90 & 41 & 92 & 37 & 92 & 36 & 85 & 45 & 82 & 45 & 79 & 44 \\
\hline New Mexico & 87 & 42 & 90 & 41 & 85 & 44 & 90 & 40 & 87 & 40 & 87 & 37 \\
\hline South Carolina & 86 & 43 & 84 & 46 & 85 & 44 & 85 & 45 & 83 & 43 & 80 & 43 \\
\hline Kentucky & 85 & 44 & 86 & 45 & 84 & 46 & 85 & 45 & 83 & 43 & 79 & 44 \\
\hline Idaho & 84 & 45 & 87 & 44 & 90 & 41 & 90 & 40 & 82 & 45 & 77 & 47 \\
\hline Louisiana & 83 & 46 & 89 & 43 & 88 & 43 & 92 & 36 & 89 & 38 & 86 & 40 \\
\hline Alabama & 82 & 47 & 81 & 48 & 83 & 48 & 83 & 48 & 81 & 48 & 75 & 49 \\
\hline Arkansas & 81 & 48 & 80 & 49 & 81 & 49 & 81 & 49 & 78 & 49 & 75 & 49 \\
\hline Oklahoma & 79 & 49 & 83 & 47 & 84 & 46 & 86 & 44 & 87 & 40 & 93 & 27 \\
\hline Mississippi & 74 & 50 & 71 & 51 & 72 & 51 & 70 & 51 & 68 & 51 & 65 & 51 \\
\hline West Virginia & 72 & 51 & 77 & 50 & 78 & 50 & 81 & 49 & 77 & 50 & 77 & 47 \\
\hline
\end{tabular}

Note: Sources, methodology, and detailed statistics are presented in a Methodological Appendix. 


\section{Table 3: Index of Fiscal Need}

National Average $=100$

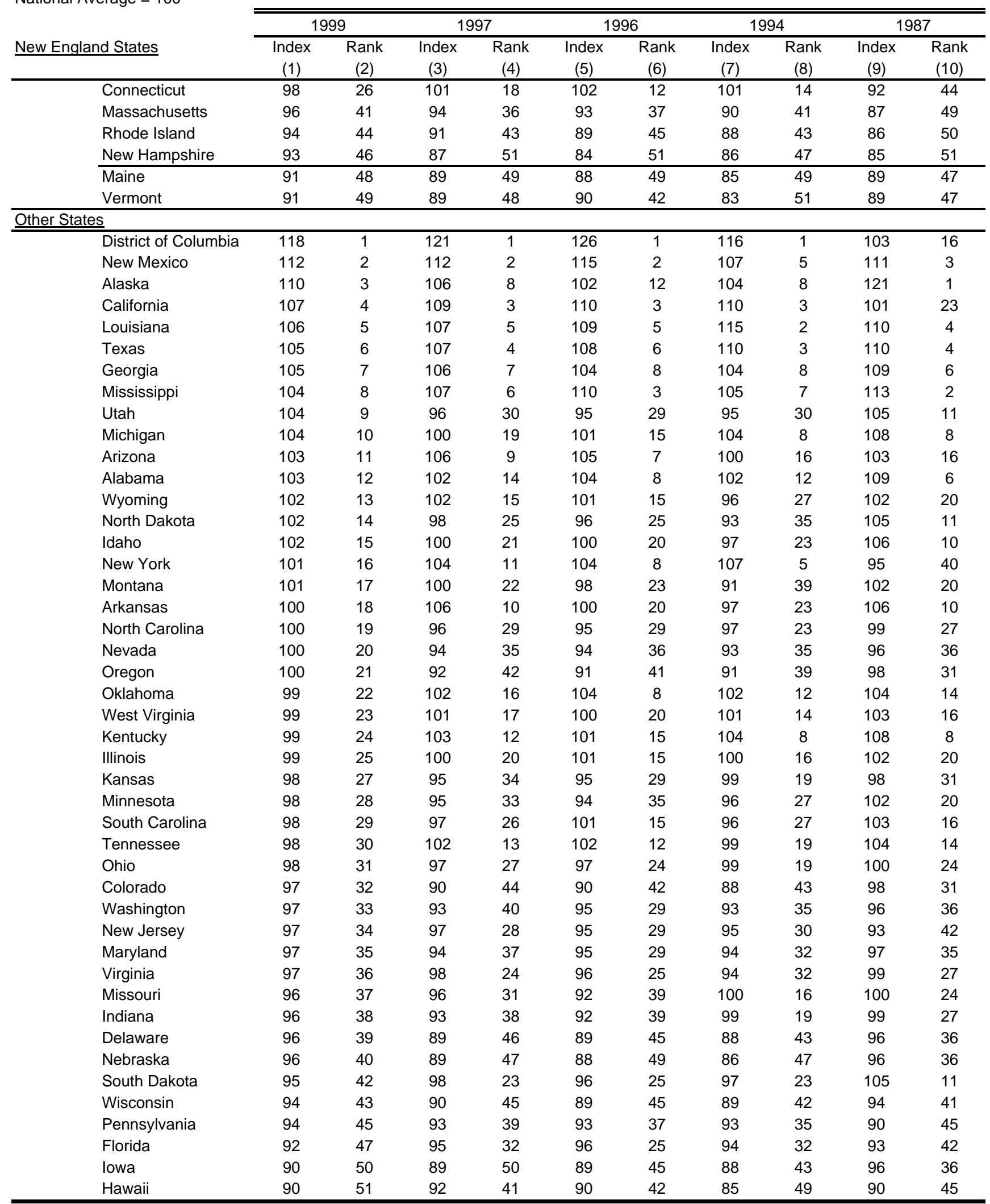

Note: Sources, methodology, and detailed statistics are presented in a Methodological Appendix. 


\section{Table 4: Index of Fiscal Comfort}

National Average $=100$

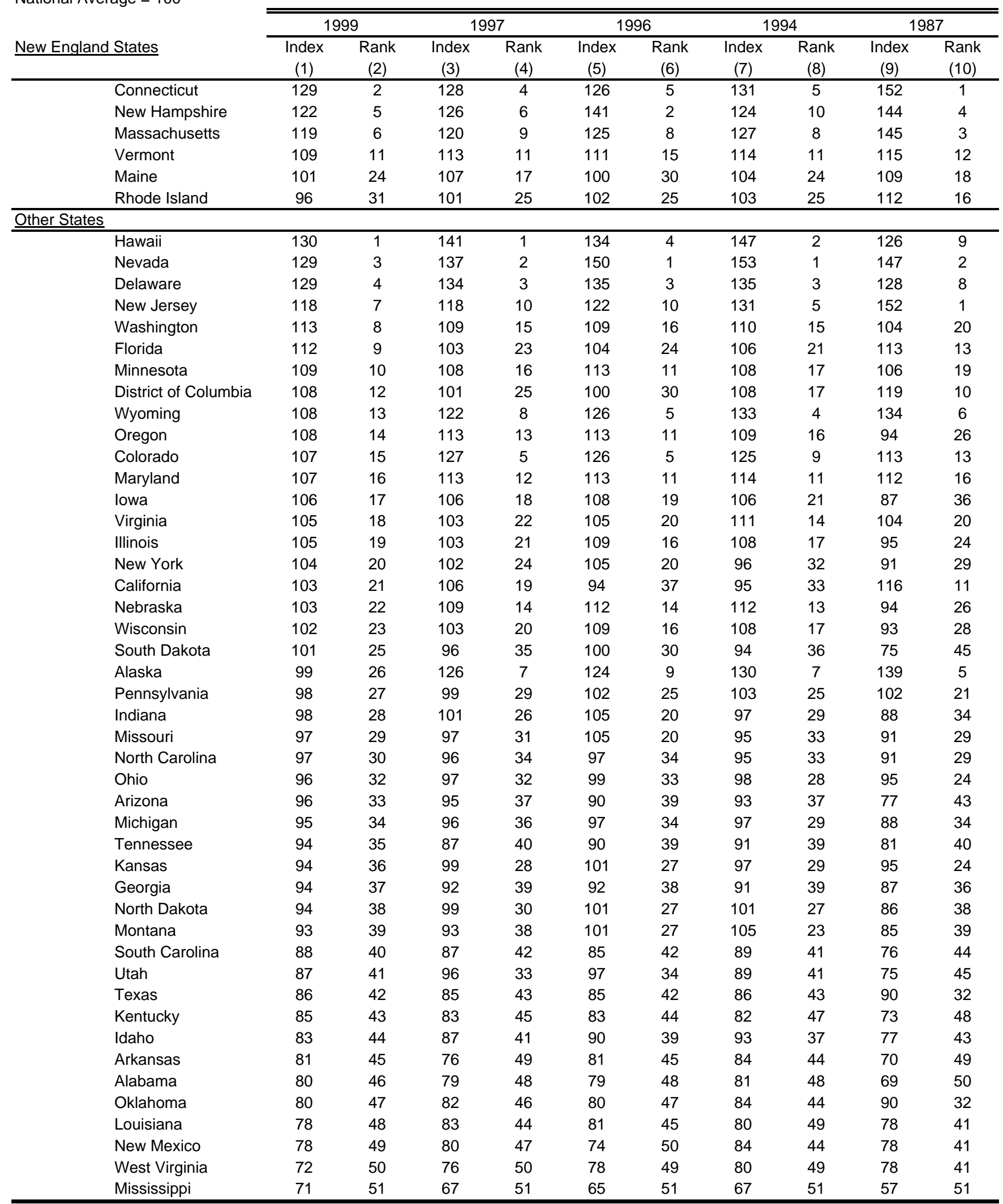

Note: Sources, methodology, and detailed statistics are presented in a Methodological Appendix. 
Table 5: Index of Tax Effort Index of Tax Effort

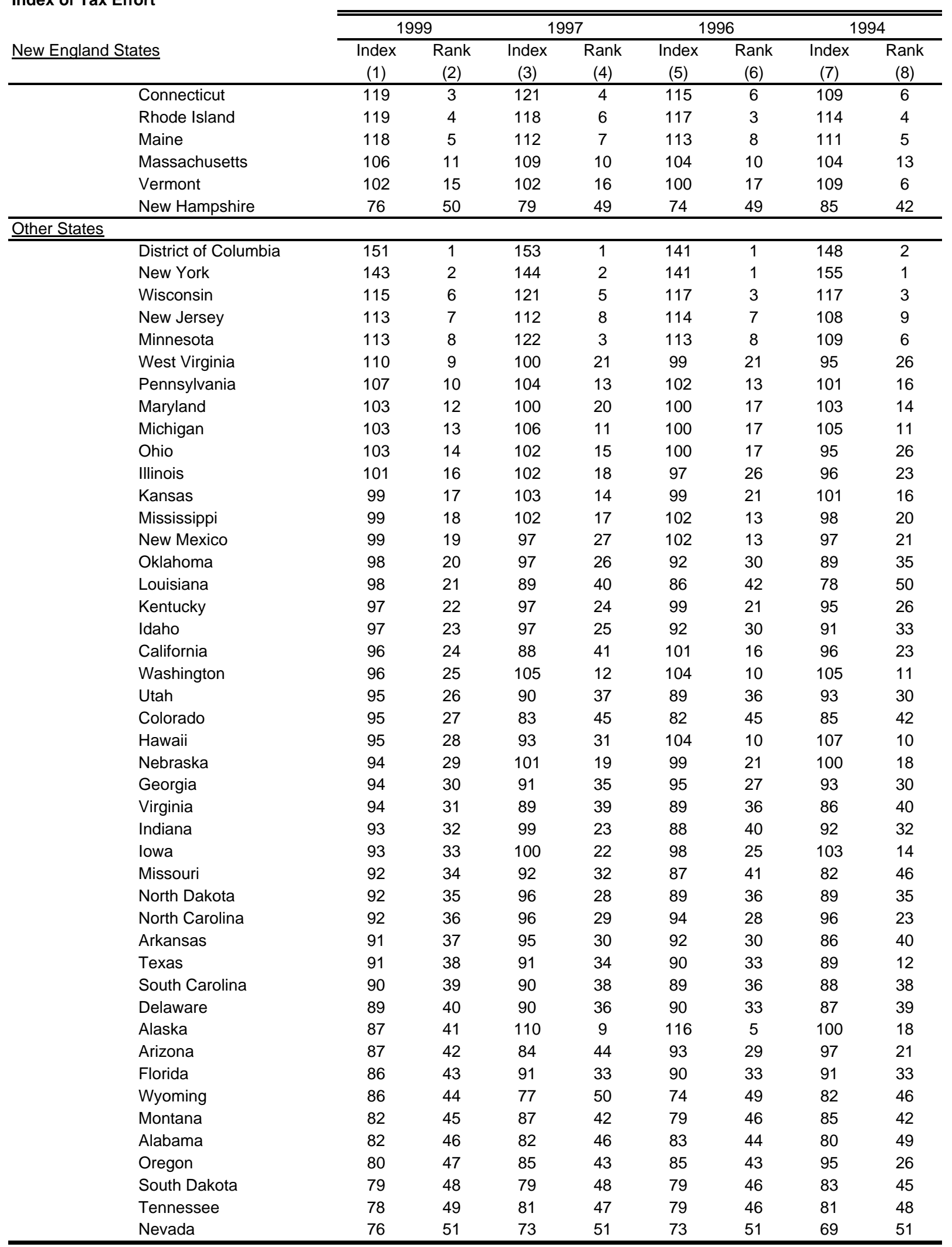

Note: Sources, methodology, and detailed statistics are presented in a Methodological Appendix. 


\section{Data Sources and Methodology fOr RePresentative TaX SYSTEM (RTS), 1999.}

This methodological appendix lays out the details of the RTS system, including all adjustments and data sources used to obtain the 1999 results. For earlier methodologies consult State Revenue Capacity and Effort, Advisory Commission on Intergovernmental Relations. (M-187. September 1993).

The RTS system establishes state tax capacity, or tax potential, by levying a standard rate on a uniform tax base for each tax in every state. The standard rate is calculated as the national total of tax collections divided by the total tax base. For instance in the 1999 data total state and local tax collections for the personal income tax were roughly \$189 billion while the standard base (defined as personal income with some adjustments, see section XIII) was roughly $\$ 5.65$ trillion. Therefore the standard rate was calculated to be 3.35 cents per dollar. Applying this rate to a given state's base, for instance Massachusetts' $\$ 170$ billion base, and dividing by state population yields a per capita capacity of about $\$ 919$. Repeating this exercise for every tax in every state and indexing each state's result to the national average creates an index of fiscal capacity (set equal to 100 for the national average). This measure reveals the ability of each state to raise tax revenue relative to the national average.

Comparing actual tax collections versus tax capacity, again indexed to the national average set to 100, creates another statistic called tax effort. This measure reveals how intensively each state taxes - both within each tax category and in total taxes.

The remainder of the appendix highlights the data sources used in calculating the standard bases and rates. Each category includes a brief description of the methodology used in constructing the tax base and notes any changes made to the data.

Data used for state and local tax collections are taken from the U.S. Census:

1999 State and Local Finance Data (including taxes and charges)

Source: U.S. Bureau of the Census, Annual Survey of Government Finances

Data for state population are taken from the U.S. Census:

1999 Population Data (based on mid-year population estimates)

Source: U.S. Bureau of the Census, Current Population Survey, "ST-99-3 State

Population Estimates: Annual Time Series, July 1, 1990 to July 1, 1999."

\section{Individual Tax Bases}

I. General Sales and Gross Receipts

Retail Sales and Selected Service Receipts by State: U.S. Bureau of the Census, Economic Census 1997. 
Gross State Product by State: U.S. Bureau of Economic Analysis, Regional Economic Accounts.

Methodology: The tax base is defined as the sum of retail trade, accommodations, food service, personal services, motion pictures, and arts and entertainment minus gas, alcohol, gambling, and non-store retail sales. For the 1997 results, data were taken from the Census of Governments. Unfortunately, since this Census publication is done every five years, much of the needed data were unavailable for 1999. As a "least-worst" method of scaling up the tax-base, each state's 1997 value was increased by its growth in gross state product (GSP). As a check on validity, and to explore other options, the 1997 bases were also scaled up by the relative growth in personal income from 1997 to 1999. This had little to no appreciable effect on the results; so GSP was chosen as the scaling factor, because it has a stronger theoretical rationale for being tied to the general sales and gross receipts tax base.

\section{Selective Sales: Motor Fuels}

Motor Fuel Volume Taxed by States: U.S. Department of Transportation, Federal Highway Administration, Highway Statistics 1999 “Table MF2: Motor Fuel Taxed 1999."

Methodology: The tax base is defined as the volume of taxed gallons of fuel for each state.

\section{Selective Sales: Public Utilities}

1999 Gas Utility Gross Revenue by State: American Gas Association, Gas Facts 2000, “Table 7-2: Gas Utility Industry Revenues, by State."

1999 Electric Utility Gross Revenue by State: Edison Electric Institute, Statistical Yearbook of the Electric Utility Industry, "Table 59: Total Electric Utility Industry, by State and Class of Service."

1999 Telephone Industry Gross Revenue by State: Federal Communication Commission, Industry Analysis Division, State-by-State Telephone Revenue and Universal Service Data, “Table 9: Industry Telephone Revenue: 1999."

Methodology: The tax base is defined as the sum of revenues of all gas, electric, and telephone companies. For the telephone industry, which includes terrestrial and wireless telephony, the state revenues are the sum of interstate and intrastate revenues. 


\section{Selective Sales: Insurance}

Property/Casualty Premiums Written by State: Insurance Information Institute, The Fact Book 2001, "Direct Premiums Written by State."

Life Insurance Premium Receipts by State: American Council on Life Insurance, Life Insurance Fact Book, 1999 E2000. “Table 9.6: Premium Receipts of U.S. Life Insurance Companies by State."

Methodology: The tax base is defined as the direct written premiums (or premium receipts) for life and property and casualty insurance.

\section{Selective Sales: Tobacco Products}

1999 State Tax-Paid Cigarette Sales: U.S. Department of Agriculture, Economic Research Service.

“Monthly State Cigarette Fiscal Tax Report - June 2000."

Methodology: The tax base is defined as the number of packages of cigarettes sold.

\section{Selective Sales: Alcoholic Beverages}

Gallons of Wine, Beer and Spirits Consumed by State: National Institute on Alcohol Abuse and Alcoholism, Surveillance Report 55E59: Apparent per Capita Alcohol Consumption: National, State and Regional Trends, 1977-1999. "Table 2: Apparent Alcohol Consumption for State, Census Regions, and the United State, 1999."

Combined State and Local Collections by Beverage Type, 1999: Distilled Spirits Council of the United States (DISCUS).

Methodology: The tax base is the combined consumption in gallons of beer, wine and distilled spirits. Census tax data has only aggregate alcohol beverage tax collections by state. A breakdown of tax collections by beverage type was obtained from DISCUS and these data were used in the calculation of the RTS rates.

\section{Selective Sales: Amusements}

1997 Retail Sales and Selected Service Receipts by State: U.S. Bureau of the Census, Economic Census 1997.

Employment Compensation in Motion Pictures and Amusements: U.S. Bureau of Economic Analysis, 2002. 
Methodology: The tax base is calculated as follows:

Tax Base $=$ Arts, entertainment and recreation + Motion Pictures and Exhibition Promoters of Performing Arts, Sports and Similar Events - Agents/Managers for Artists, Athletes and Other Public Figures - Independent Artists, Writers and Performers - Coin Operated Amusement Devices (Except Slots).

Since 1997 data were obtained from the Economic Census (done only every five years), the data were adjusted by income growth in amusement employment categories.

The individual adjustment rates appear in the appendix tables.

\section{Selective Sales: Pari-mutuels}

1999 Total Pari-mutuel Handle ARCI, Inc., provided by Christiansen Capital Advisors LLC.

Methodology: Tax base is defined as the sum of dog racing, horse racing and jailai handles within each state.

\section{Licenses: Motor Vehicle Registrations}

Counts of Registered All Motor Vehicles and Motorcycles (Private and Commercial Vehicles only) U.S. Department of Transportation, Federal Highway Administration, Highway Statistics 1999 "Table MV-2: State MotorVehicle Registrations."

Methodology: Tax base is defined as the sum motor vehicle registrations in the state.

\section{Licenses: Corporations}

Number of Corporate Income Tax Returns Internal Revenue Service, Internal Revenue Service Data Book, "Table 4: Number of Returns Filed, by State."

Methodology: The tax base is defined as total number of corporation licenses granted in the state. Separate number did not exist for Maryland and District of Columbia, so they were separated using the personal income ratio.

\section{Licenses: Motor Vehicle Operators}

Number of Licensed Drivers by State U.S. Department of Transportation, Federal Highway Administration, Highway Statistics 1999, “Table DL-22: Total Licensed Drivers, by Age." 
Methodology: Tax base is the number of licenses in each state.

\section{Licenses: Fishing and Hunting}

Number of Fishing and Hunting Licenses by State U.S. Department of the Interior, Fish and Wildlife Services, Division of Federal Aid, State and Fish Game Departments, "Tables: Number of Paid Fishing License Holders, License Sales, and Cost to Anglers - Fiscal Year 1999 and Number of Paid Hunting License Holders, License Sales and Cost to Hunters-Fiscal Year 1999."

Methodology: The tax base is defined as total number of licenses granted by each state.

\section{Individual Income Tax}

Adjusted Gross Income, Adjustments and Adjustments for Residency by State and Number of Dependents, Single Returns, Joint Returns and Head of Household Returns Internal Revenue Service, Statistics of Income, "Individual Income Tax Information by State."

Exemption Amounts for Singles Returns, Joint Returns, Head of Household Returns and Dependents Government of the District of Columbia, Tax Rates and Tax Burdens in the District of Columbia- A Nationwide Comparison, "Table 15: Individual Income Tax."

Methodology: The tax base is defined as adjusted gross income (AGI) using the adjustment amounts and the adjustments for residency net of exemptions.

Individual Income Tax Base $=$ AGI + Adjustments - Adjustments for Residence Exemptions

The values of exemptions were obtained in a multi-step process:

1. Found the exemption level for each type of exemption by state.

2. Calculated the weighted average of the exemption level for all of states for each type of exemption. The weight used was per capita individual income tax receipts.

3. Used this weighted average to calculate amount of exemptions by state. Multiplied the weighted average exemption value by the number of exemptions, by exemption type, by state. Added amount of all exemptions together for each state.

\section{Corporate Income Tax}




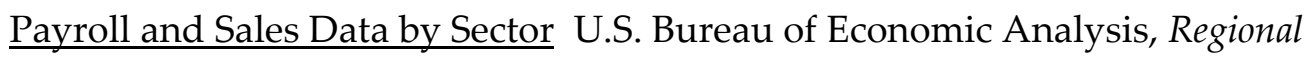
Accounts

Data, Table SA07

Corporate Profits U.S. Bureau of Economic Analysis, National Income and

Product Accounts, "Table 6.17c: Corporate Profits by Industry Group."

Methodology: The tax base is defined as corporate profits. However these data are not available on the state level, so the national data was allocated to the states. This involved a multi-step process:

1. 1997 Annual Receipts and Payroll data by state by industry were collected from the Economic Census. 1999 corporate profits by industry were collected on the national level from the BEA. 1999 wages and salary disbursements by state by industry were collected from the BEA.

2. Estimated the 1999 receipts by state by industry using the following formula: 99 receipts $=99$ payroll $*$ (97 receipts / 97 payroll)

3. Calculated the percent of each industry located in each state. For each industry, the state's percentage of the national aggregate receipts and payroll respectively were calculated.

4. Used the percentages calculated above to figure out how much of national corporate profits should be allocated to each state. For each industry, the receipts were weighted once and payroll twice. All industries for each state were then added together to end with an estimate of corporate profits by state.

\section{Property Tax}

1999 Market Value of Property by State Called revenue officials, went to State web sites, and read various studies to find the market value of property by state. All contacts are listed in the above spreadsheet 1997 Farm Real Estate Values and Property Taxes

U.S. Department of Agriculture, National Agricultural Statistics Service: 1997 Census of Agriculture, Vol. 1. 1999 Gas Utility Miles of Pipeline American Gas Association, Gas Facts: 1997 Data, “Table 5-2: Gas Utility Industry Miles of Pipeline and Main, by State, 1980-1999." 1999 Electric Utility Generating Capacity Edison Electric Institute: Statistical Yearbook of the Electric Utility Industry 1997, "Table 4: Installed Generating Capacity: Investor-Owned Electric Utilities by State."

1999 Total Access Telephone Lines

Federal Communications Commission, Common Carrier Bureau, 1997 Statistics of Communication Common Carriers: “ Table 2.5- Access Lines by Type of Customer For Reporting Local Exchange Carriers." 1999 Assets of Active Corporations by Industry 
Internal Revenue Service, Statistics of Income 1997, Table 1: 1999 Returns of Active Corporations."

1999 Corporate Depreciable Assets, Depletable Assets and Land

Internal Revenue Service, Statistics of Income 1997, "Table 6: Returns of Active

Corporations."

1999 Personal Income by Industry

Bureau of Economic Analysis, "Table SA05: Personal Income by Major Source and Earnings by Industry."

1990 Owner-Occupied Housing Aggregate Values by State

U.S. Census Bureau Decennial Census, Summary Tape File 3 on CD-ROM,

"Table H-62: Aggregate Value by Mortgage Status."

1990 Aggregate Gross Rent by State

U.S. Census Bureau Decennial Census, Summary Tape File 3 on CD-ROM,

"Table H-44: Aggregate Gross Rent"

1990-1999 New Construction by State

Statistical Abstract of the United States (various years), Table entitled "Construction

Contracts - Value by State"

1990-1999 Conventional Mortgage Home Price Index

Freddie Mac Index, http://www.freddiemac.com/finance/cmhpi/release.htm

Value of Construction to Total Value

U.S. Bureau of Census, Characteristics of New Housing: 1993, 1995, 1996 and 1998.

Tables entitled "Average and Median Square Feet of Floor Area, by Category of

House, Location and Type of Financing", "Sales Price of Houses, by Location and Type of Financing", and "Price per Square Foot of Floor Area, by Location".

Methodology: The tax base is defined as the total property value in the state.

Total property value consists of farm property, residential property, corporate property and utility property including all exemptions. The goal was to construct a base that represented the total potential property tax base in the state. This involved aggregating the following classes of property:

1. Utility property: the sum of the gas, electric and telephone industries. Assets for each of these industries were allocated by state based on the state's share of pipeline, generating capacity and telephone lines, respectively.

2. Corporate property: consists of depreciable assets, depletable assets, and land (minus accumulated depreciation). Corporate property was allocated across states using methodology similar to that used for corporate income tax. Property was allocated across states using the percentage of personal income by industry for each state.

3. Residential property: in theory this class of property should include all residential property in the state-including expeditions. The estimate was obtained by:

i) Calculating the growth in the Freddie Mac index was calculated for each state from 1997-1999. 
ii) Using this growth rate to inflate 1997 Total Aggregate OwnerOccupied Housing Property Values and the 1997 Asset Value from Rental Property to 1999 levels.

iii) Residential Construction contracts were inflated to include land value for each year (1997-1999). This was done by dividing the Residential Construction Contracts by the ratio of construction to total property value. This ratio was calculated by first finding the average value of property by multiplying average square feet of floor area by the average price per square foot, by region. This product was then divided by the average sales price of one-family houses to arrive at the value of construction to total value.

iv) The Residential Construction contracts (inflated to include land value), were then inflated using the Freddie Mac Index, by year. The values for each year were then summed to arrive at a total value for residential property by state.

Estimates for total property value from this method were then compared with estimates of property value obtained from state agencies. Since the agencies vary widely on the data they report, some include the total value of all property, others of only taxable property, and some only the accessed value of taxable property, it was not clear that state estimates were always the preferred measure. However, many estimates were similar to the obtained results, though several were substantially different. Consequently, faced with two estimates, the larger value was chosen. In other words, when faced with the improbable result that the estimated value of potentially taxable property was less than the reported taxed property, the latter was taken as a superior measure.

Estimating the property tax base in California is more problematic and required an additional step. Because Proposition 13 limits the annual increase in the assessed value of any taxable property to one percent a year, unless ownership of the property changes hands, in which case the property is reassessed at its purchase price it is hard to obtain the "market value" of real estate (see text). The estimation of California's property tax base was made as follows. First, it was assumed that the statewide ratio of assessed to fair market value was the same as that estimated for Los Angeles County in FY1996 by Sheffrin and Sexton (1998). Next the "extrapolated" estimate (see methodology outlined above) was compared with the one based on the ratio estimated by Sheffrin and Sexton and the larger of the two was used, because historically the extrapolation technique seemed to be producing estimates that were too low. In FY1997, the estimate based on the Sheffrin and Sexton ratio was larger, while in FY1999 the "extrapolated" estimate was larger.

\section{Estate and Gift Tax}


Federal Estate and Gift Tax Collections Internal Revenue Service, 1999 Internal Revenue Service Data Book, "Table 8: Tax Collections, by State."

State Estate and Gift Tax Collections U.S. Census Bureau, State and Local Government Tax Collections.

Methodology: The tax base is defined as the federal estate and gift collections. As the federal taxes are applied uniformly across all states, this should give a valid measure of the magnitude of each state's base. The data for Maryland and District of Columbia collections separated using the personal income ratio.

\section{Severance Taxes}

Value of Oil Production Energy Information Administration, Petroleum Marketing Monthly January 1999, "Table 21: Domestic Crude Oil First Purchase Prices."

Energy Information Administration, Petroleum Supply Annual 1997, "Table 14: Production of Crude Oil by PAD District and State."

Value of Coal Production Energy Information Administration, Coal Industry Annual 1999 Data Tables, "Table 1: Coal Production by State." and "Table 80: Average Mine Price of Coal by State."

Value of Natural Gas Production Energy Information Administration, Historical Natural Gas Annual 1999, "Table 6: Wellhead Value and Marketed Production of Natural Gas by State, 1967-1999."

Value of Nonfuel Mineral Production Energy Information Administration, Minerals Yearbook 1999, “Table 5: Nonfuel Mineral Production in the United States, by State."

Methodology: Tax Base is the sum of the value of oil production, coal production, natural gas production and nonfuel mineral production.

\section{All Other Taxes}

Personal Income U.S. Bureau of Economic Analysis, Regional Accounts Data, “Table SA-05: Personal Income by State, 1999."

Methodology: Tax Base is Personal Income. Tax revenue for All Other Taxes consists of the following categories of tax revenue from Census of Governments (see I. State and Local Finance Data above for source information): Other Selective Sales (T-29), Amusement Licenses (T-21), Alcohol Licenses (T-20), Public Utility Licenses (T-27), Occupational/Business Licenses (T-28), Other Licenses (T-19), Documentary and Stock Transfers (T-51), and Not Elsewhere Classified (T-99). 


\section{Data Sources and Methodology for RePresentative EXPENDITURE SYSTEM (RES), 1999.}

This methodological appendix spells out the details of the RES system, including all data sources, workload measures and adjustments used to obtain the 1999 results. For earlier methodologies see Robert Rafuse's Representative Expenditures: Addressing the Neglected Dimension of Fiscal Capacity, Advisory Commission on Intergovernmental Relations, M174, December 1990.

The RES system provides normalized expenditure estimates for a given unit of public services provided. This involves several steps.

First, it is necessary to identify and define categories of expenditures that are principally influenced by factors other than state population. Six such categories were identified: elementary and secondary education, higher education, public welfare, health and hospitals, highways, and police and corrections. In 1999, these categories accounted for just under 71 percent of all direct general expenditures for state and local governments.

Next, it is necessary to define measurable "workload" factors--other than the price of inputs--that affect the cost of providing the service. For example, the workload measures for highway expenditures are the number of vehicle miles traveled, measured maintenance costs due to traffic, total lane miles, and measured maintenance costs due to time and exposure. When more than one workload measure is employed, a weighted average is used to calculate the final composite workload. In this example, based on engineering estimates, vehicle miles traveled is weighted 4.71 times more than total lane miles. In this way, workloads are constructed for each expenditure category.

The national total expenditure level is then multiplied by each workload factor in each tax to estimate what it would have cost the state to provide one workload-measure unit of services. This is done across all expenditure categories to create a total expenditure estimate. However, to fully determine the state's fiscal need index, it is necessary to adjust for input prices across states. In general, this is done by controlling for the prices of inputs used in the provision of public services; the particulars of this methodology are outlined below in the input-cost section.

For each state, per capita expenditures are summed to obtain total per capita expenditures. Indexing these to the national average (set to 100) gives each state an index of fiscal need - the primary index used in the RES system.

The remainder of this Appendix details the data sources and workloads used in the RES 1999 results. 


\section{State and Local Expenditure Data}

U.S. Bureau of the Census, Annual Survey of Government Finances.

\section{Input-Cost Index Data}

Earnings by Occupation and Education: 2000 Census. Available online: http://www.census.gov/hhes/income/earnings/earnings.html March 1999 State and Local Government Payroll Data by Function: Bureau of the Census, State and Local Government Employment and Payroll- 1999.

Methodology: The input-cost adjustment normalizes for cost differentials across states. The process used the 2000 Census (1999 data) to tabulate earnings by state by educational attainment for males aged 45-54 who are year-round, full-time workers. Next, state-by-state labor cost indexes were created controlling for education level by weighting each state's median earnings by education level by the national distribution of educational attainment. From this, an input-cost index for each state by function was constructed. This was done by annualizing the March payroll and then dividing this by total functional expenditures, so that (at the national level) each functional level had payroll costs as a percentage of the total costs. This payroll percentage was turned into a compensation percentage by dividing payroll percentages by 0.75 , on the assumption that payroll represented 75 percent of total compensation. Finally, for each function, the compensation percentage was multiplied by the labor cost index by state and added to the non-compensation percentage of costs multiplied by 100 .

For example,

Input Cost Index $=\left(\operatorname{comp} \%{ }^{*}\right.$ labindex $)+\left((1-\operatorname{comp} \%)^{*} 100\right)$,

where

comp $\%=$ compensation as percentage of total function cost,

and

labindex $=$ state's labor cost index.

\section{Calculating Representative Expenditures}

To calculate representative expenditures by function, multiply: (state's workload measure by function $)^{*}$ (total national direct general expenditure by function $)^{*}\left(\right.$ state's $^{\prime}$ input cost index by function). These numbers are then normalized so that total representative expenditures $=$ actual direct general expenditures. 


\section{Data for Estimating Representative Expenditures}

\section{Public Welfare}

Total 1999 Population: U.S. Census Bureau, Current Population Survey, "ST-99-3 State Population Estimates: Annual Time Series, July 1, 1990 to July 1, 1999." Average of 1998-1999 Poverty Rates: U.S. Census Bureau, Current Population Survey, "Historical Poverty Table 19"

Methodology:

Workload measure $=$ State's share of total population in poverty.

\section{Elementary and Secondary Education}

Elementary and Secondary Age Population: U.S. Census Bureau, State Population Estimates, "Table ST-99-12: Population Estimates for the U.S. and States by Single Year of Age."

Percentage of People Under 18 Living in Poverty: U.S. Census Bureau, Current Population Survey, March Supplement, "Table 25: Poverty Status by State, 1999" Fall 1999 Private School Enrollment by State: U.S. Department of Education, National Center for Education Statistics, Digest of Education Statistics, 2000. "Table 63: Private elementary and secondary schools, enrollment, teachers and high school graduates, by state, Fall 1999."

\section{Methodology:}

The overall ratios of elementary and secondary education population were used to divide total private school enrollment between elementary and secondary. The assumptions used by Rafuse--that cost per pupil for elementary students is 60 percent of that for secondary students and that children in poverty cost 25 percent more to educate--are used. Thus, the Education Cost Index $=[1+$ $\left(0.25^{*}\right.$ Under 18 Poverty Rate $){ }^{*}\left[\left(0.6^{*}\right.\right.$ Elementary public school enrollment $)+$ (Secondary public school enrollment)].

Workload measure $=$ State's share of education cost index.

\section{Higher Education}

1999 State Population Aged 14-17, 18-24, 25-34, and 35+: U.S. Census Bureau, State Population Estimates, "Table ST-99-12: Population Estimates for the U.S. and States by Single Year of Age.

Full-Time and Part-Time Students Enrolled in Institutions of Higher Education in Fall 1999, by Age Groups 14-17, 18-24, 25-34, 35+: U.S. Department of Education, National Center for Education Statistics, Digest of Education Statistics. "Table 175: Total fall enrollment in degree-granting institutions, by attendance status, sex, and age: 1970-2000."

Assumed three part-time students $=1$ full-time equivalent (FTE). 
Methodology: Determined estimated college population by weighting state's population of each group by national percentage of each group enrolled in institutions of higher education.

Workload measure $=$ State's share of estimated college population .

\section{Health and Hospitals}

Total 1999 Population U.S. Census Bureau, Current Population Survey, "ST-99-3 State Population Estimates: Annual Time Series, July 1, 1990 to July 1, 1999." Average Income of the Bottom (Fifth) and Next-to-Bottom (Fourth) Quintile of Families, by State: Economic Policy Institute/Center on Budget and Policy Priorities, Pulling Apart: A State-by-State Analysis of Income Trends, January 2000 Appendix Table 5.

1999 Number of Disabled Workers by State: Social Security Administration "Table 4: Number of OASDI beneficiaries with benefits in current-payment status, by type of benefit, by sex of beneficiaries aged 65 and older, and by State and County, December 1999."

Methodology: Instead of using the percentage of people under 150 percent of poverty, which was used in the earlier studies, the nation's income average among the fourth and fifth quintiles was taken as a percent of the states' average. This percentage was then multiplied by the state's share of population to yield the final measure of poverty for the lowest two quintiles by state.

For example,

State's share of low-income families $=[($ nation's avg $5+$ nation's avg4)/2] $/($ state's avg $5+$ states's avg 4$) / 2]^{*}$ (state's share of population), where

avg $5=$ the average of the bottom quintile, and $\operatorname{avg} 4=$ the average of the next-to-bottom quintile.

Workload measure = Equally weighted average of state's share of total population, state's share of work-disabled population, and state's share of lowincome families.

\section{Highways}

1999 Annual Vehicle-Miles of Travel: Federal Highway Administration, Highway Statistics 1999: "Table VM-2A: Annual Vehicle-Miles of Travel 1999." 1999 Lane Mileage: Federal Highway Administration, Highway Statistics 1999:

“Table HM-60: Estimated Lane Mileage by Functional System 1999." 
Methodology:

Workload measure $=(82.5 \%$ * State's share of Vehicle Miles $)+(17.5 \%$ * State's share of Lane Mileage).

Weightings based on GAO Report Cited in Rafuse study.

VI. Police and Corrections

Total 1999 Population U.S. Census Bureau, Current Population Survey, "ST-99-3

State Population Estimates: Annual Time Series, July 1, 1990 to July 1, 1999."

State Population Aged 18-24: U.S. Census Bureau, State Population Estimates, Table ST-99-12: Population Estimates for the U.S. and States by Single Year of Age.

1999 Number of Murders by State: Federal Bureau of Investigation, 1999 Crime in the United States "Table 5: Index of Crime: Region, Geographic Division, and State."

Methodology:

Workload measure $=$ Equally weighted average of state's share of total population, state's share of population aged 18-24, and state's share of murders.

VII. Other Categories of Expenditures: Environment and Housing, Interest on General Debt, Governmental Administration, and All Other Direct General Expenditures

Methodology: Workload Measure defined as state's share of total 1999 U.S. population 
Summary Data: Representative Tax System

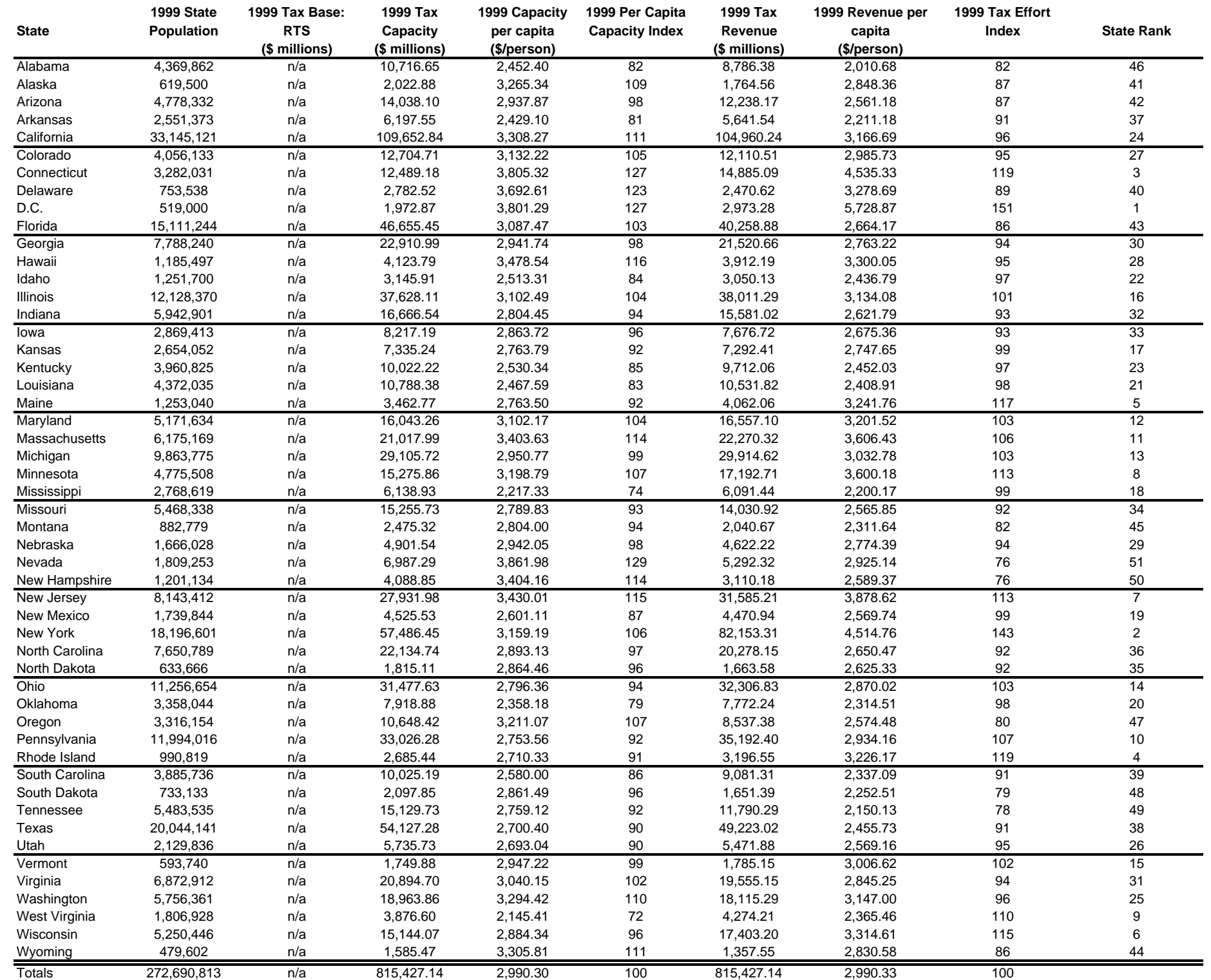

RTS rate see individual bases 
Summary Data: Capacity

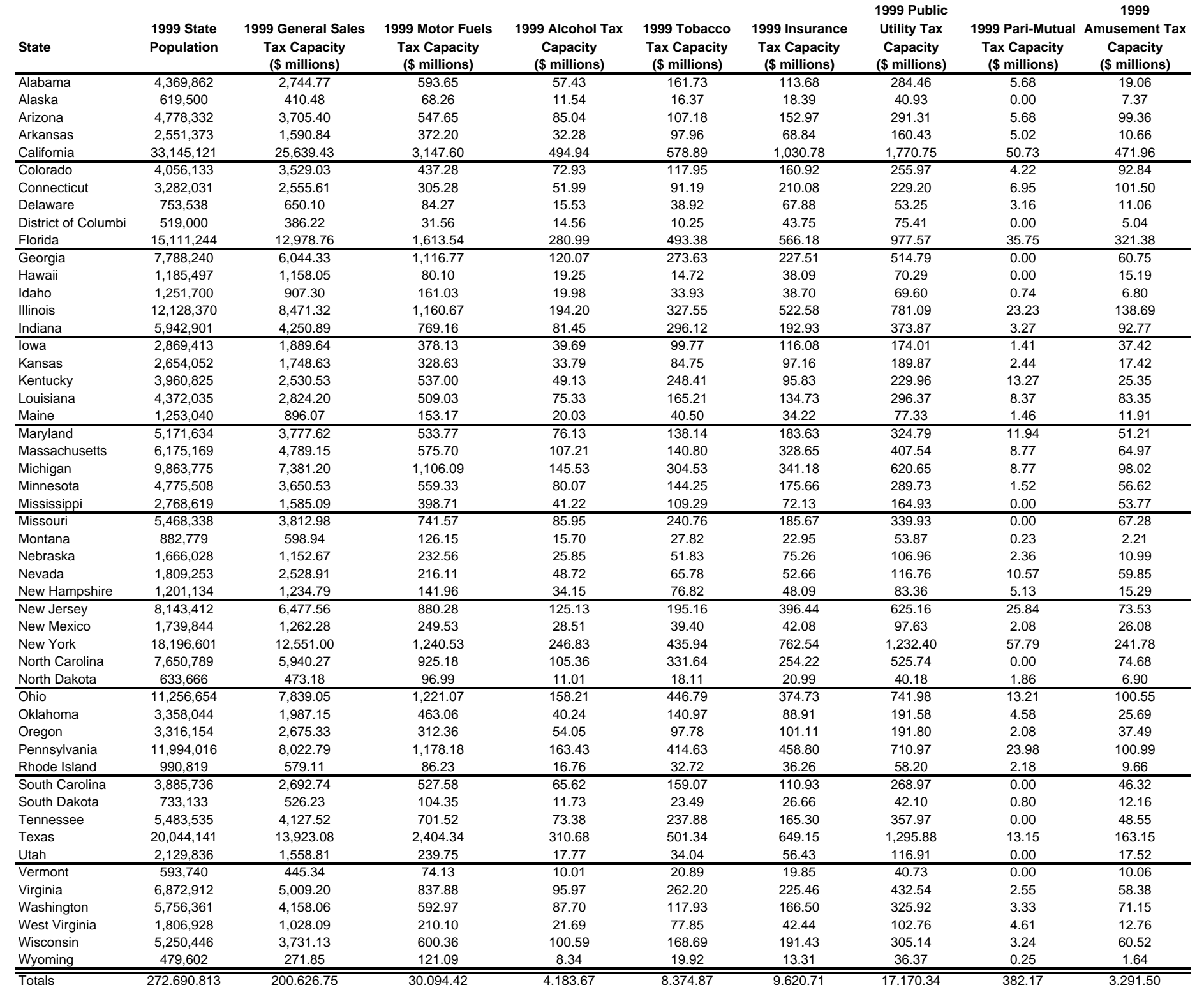


Summary Data: Capacity (Continued)

\begin{tabular}{|c|c|c|c|c|c|c|c|c|c|c|c|}
\hline State & $\begin{array}{l}1999 \text { State } \\
\text { Population }\end{array}$ & $\begin{array}{l}1999 \text { Motor Vehicle } \\
\text { LicenseTax } \\
\text { Capacity } \\
(\$ \text { millions }) \\
\end{array}$ & $\begin{array}{l}1999 \text { Motor Vehicl } \\
\text { Operator License } \\
\text { Tax Capacity } \\
\text { (\$ millions) }\end{array}$ & $\begin{array}{l}1999 \text { Hunting and } \\
\text { Fishing License } \\
\text { Tax Capacity } \\
\text { (\$ millions) }\end{array}$ & $\begin{array}{l}1999 \text { Corporate } \\
\text { License Tax } \\
\text { Capacity } \\
\text { ( } \$ \text { millions) } \\
\end{array}$ & $\begin{array}{l}1999 \text { Personal } \\
\text { Income Tax } \\
\text { Capacity } \\
\text { (\$ millions) } \\
\end{array}$ & $\begin{array}{l}1999 \text { Corporate } \\
\text { Income Tax } \\
\text { Capacity } \\
\text { ( } \$ \text { millions) } \\
\end{array}$ & $\begin{array}{l}1999 \text { Tax } \\
\text { Property } \\
\text { Capacity } \\
\text { (\$ millions) } \\
\end{array}$ & $\begin{array}{l}1999 \text { Estate and } \\
\text { Gift Tax } \\
\text { Capacity } \\
(\$ \text { millions }) \\
\end{array}$ & $\begin{array}{c}1999 \text { Severance } \\
\text { Tax Capacity } \\
\text { (\$ millions) }\end{array}$ & $\begin{array}{l}1999 \text { Other Tax } \\
\text { Capacity } \\
\text { ( } \$ \text { millions) } \\
\end{array}$ \\
\hline Alabama & $4,369,862$ & 279.88 & 23.86 & 12.94 & 71.27 & $2,280.71$ & 417.33 & $2,927.77$ & 74.62 & 78.27 & 569.55 \\
\hline Alaska & 619,500 & 40.26 & 3.18 & 14.04 & 11.90 & 461.09 & 57.69 & 611.32 & 3.95 & 145.61 & 100.48 \\
\hline Arizona & $4,778,332$ & 238.99 & 22.52 & 16.77 & 98.90 & $2,948.14$ & 501.52 & $4,357.62$ & 115.33 & 69.27 & 674.43 \\
\hline Arkansas & $2,551,373$ & 127.66 & 13.33 & 21.32 & 51.84 & $1,251.51$ & 247.29 & $1,753.48$ & 43.48 & 32.11 & 317.32 \\
\hline California & $33,145,121$ & $1,857.19$ & 143.32 & 63.76 & 516.26 & $\begin{array}{l}23,967.63 \\
23\end{array}$ & $3,834.80$ & $39,320.31$ & $1,014.54$ & 176.30 & $5,573.64$ \\
\hline Colorado & $4,056,133$ & 266.40 & 20.59 & 24.61 & 130.06 & $3,213.40$ & 574.39 & $2,934.57$ & 86.83 & 68.84 & 713.88 \\
\hline Connecticut & $3,282,031$ & 196.79 & 16.38 & 5.24 & 80.00 & $3,279.54$ & 566.54 & $3,893.30$ & 158.35 & 2.31 & 738.93 \\
\hline Delaware & 753,538 & 44.08 & 3.81 & 1.08 & 25.74 & 606.99 & 221.30 & 795.24 & 30.85 & 0.27 & 128.99 \\
\hline District of Columbi & 519,000 & 15.90 & 2.42 & 0.00 & 16.20 & 477.76 & 203.41 & 549.64 & 22.04 & 0.00 & 118.71 \\
\hline Florida & $15,111,244$ & 807.94 & 84.71 & 38.59 & 635.71 & $10,440.60$ & $1,482.07$ & $12,797.60$ & 650.30 & 48.69 & $2,401.70$ \\
\hline Georgia & $\frac{7,111,249}{7,788,240}$ & 495.78 & 37.41 & 27.68 & 196.15 & $5,102.38$ & $\frac{1,46.01}{1,032.70}$ & $\frac{1,1}{6,267.20}$ & 156.24 & 41.20 & $\frac{2,40.10}{1,196.40}$ \\
\hline Hawaii & $1,185,497$ & 51.35 & 5.20 & 0.26 & 30.20 & 730.80 & 107.38 & $1,585.31$ & 29.03 & 2.10 & 186.47 \\
\hline Idaho & $1,251,700$ & 81.38 & 6.02 & 23.59 & 28.08 & 651.11 & 106.24 & 825.74 & 13.27 & 10.53 & 161.89 \\
\hline Illinois & $12,128,370$ & 677.85 & 54.19 & 28.19 & 309.64 & $9,308.77$ & $1,816.49$ & $11,237.33$ & 398.79 & 48.92 & $2,128.62$ \\
\hline Indiana & $5,942,901$ & 391.13 & 27.16 & 21.67 & 120.25 & $3,705.73$ & 735.65 & $4,585.54$ & 105.12 & 35.30 & 878.54 \\
\hline lowa & $2,869,413$ & 224.41 & 13.47 & 21.31 & 62.45 & $1,701.63$ & 347.64 & $2,642.51$ & 38.19 & 12.35 & 417.07 \\
\hline Kansas & $2,654,052$ & 157.12 & 12.98 & 12.78 & 53.79 & $1,652.99$ & 309.27 & $2,121.82$ & 62.45 & 50.49 & 398.86 \\
\hline Kentucky & $3,960,825$ & 197.30 & 18.38 & 21.97 & 73.93 & $2,108.56$ & 413.15 & $2,782.28$ & 56.61 & 101.75 & 518.83 \\
\hline Louisiana & $4,372,035$ & 246.74 & 19.07 & 26.15 & 101.12 & $2,205.36$ & 413.38 & $2,693.15$ & 88.74 & 329.68 & 568.42 \\
\hline Maine & $1,253,040$ & 66.89 & 6.33 & 7.89 & 31.77 & 719.19 & 115.68 & $1,080.17$ & 23.81 & 2.39 & 173.98 \\
\hline Maryland & $\frac{1,250,040}{5,171,634}$ & 274.16 & 22.10 & $\frac{1.05}{14.61}$ & 132.13 & $4,155.35$ & $\begin{array}{l}11.06 \\
534.36\end{array}$ & $\frac{1,00.11}{4,680.44}$ & $\frac{2.01}{179.82}$ & $\frac{2.05}{10.50}$ & $\begin{array}{l}17.90 \\
942.57\end{array}$ \\
\hline Massachusetts & $6,175,169$ & 379.26 & 30.57 & 9.76 & 157.61 & $5,677.13$ & 971.94 & $5,903.85$ & 238.86 & 4.87 & $1,221.36$ \\
\hline Michigan & $9,863,775$ & 589.76 & 47.39 & 63.99 & 221.23 & $6,775.76$ & $1,268.12$ & $8,305.47$ & 209.13 & 54.86 & $1,564.03$ \\
\hline Minnesota & $4,775,508$ & 299.52 & 20.03 & 42.09 & 122.69 & $3,648.77$ & $\begin{array}{c}1,20.12 \\
695.92\end{array}$ & $4,534.98$ & 84.72 & 38.75 & 830.69 \\
\hline Mississippi & $2,768,619$ & 163.43 & 12.30 & 12.74 & 43.37 & $1,206.46$ & 221.70 & $1,678.33$ & 37.78 & 14.08 & 323.59 \\
\hline Missouri & $5,468,338$ & 316.13 & 26.49 & 43.02 & 113.01 & $3,464.15$ & 682.61 & $4,147.69$ & 137.54 & 32.94 & 818.02 \\
\hline Montana & 882,779 & 70.98 & 4.53 & 21.48 & 26.56 & 444.06 & 63.21 & 846.34 & 10.71 & 28.68 & 110.92 \\
\hline Nebraska & $1,666,028$ & 109.79 & 8.28 & 14.04 & 42.44 & $1,073.23$ & 199.55 & $1,501.90$ & 33.35 & 4.18 & 256.30 \\
\hline Nevada & $1,809,253$ & 85.29 & 8.90 & 6.22 & 54.25 & $1,448.97$ & 214.97 & $1,619.01$ & 65.06 & 74.42 & 310.82 \\
\hline New Hampshire & $1,201,134$ & 77.08 & 6.33 & 7.10 & 30.39 & 883.31 & 133.12 & $1,074.83$ & 26.39 & 1.52 & 209.19 \\
\hline New Jersey & $\frac{1,201,104}{8,143,412}$ & 422.45 & 38.54 & 9.10 & $\frac{20.55}{281.28}$ & $6,924.89$ & $1,198.66$ & $\frac{1,04.05}{8,360.31}$ & 248.55 & $\begin{array}{ll}1.02 \\
7.07\end{array}$ & $1,641.77$ \\
\hline New Mexico & $1,739,844$ & 113.24 & 8.41 & 8.50 & 30.34 & 870.12 & 116.79 & $1,252.42$ & 31.64 & 130.75 & 215.73 \\
\hline New York & $18,196,601$ & 754.54 & 73.45 & 44.78 & 586.98 & $15,201.14$ & $3,187.24$ & $16,562.02$ & 791.87 & 25.18 & $3,490.44$ \\
\hline North Carolina & $7,650,789$ & 412.43 & 38.23 & 19.82 & 159.20 & $4,821.02$ & $1,000.37$ & $6,197.83$ & 167.81 & 18.29 & $\begin{array}{l}3,40.44 \\
1,142.65\end{array}$ \\
\hline North Dakota & 633,666 & 49.46 & 3.17 & 10.06 & 13.31 & 347.87 & 60.07 & 549.02 & 5.87 & 21.56 & $\begin{array}{l}85.50 \\
8.12\end{array}$ \\
\hline Ohio & $11,256,654$ & 733.22 & 55.44 & 39.04 & 217.91 & $7,346.54$ & $1,431.94$ & $8,588.12$ & 389.38 & 52.03 & $1,728.45$ \\
\hline Oklahoma & $3,358,044$ & 208.35 & 16.01 & 14.05 & 72.83 & $1,662.14$ & 278.98 & $2,100.61$ & 68.65 & 114.86 & $\begin{array}{c}1,120.45 \\
440.21\end{array}$ \\
\hline Oregon & $3,316,154$ & 214.68 & 16.92 & 40.28 & 79.28 & $2,188.71$ & 361.42 & $3,704.16$ & 58.90 & 7.67 & 504.42 \\
\hline Pennsylvania & $11,994,016$ & 650.17 & 58.55 & 71.10 & 220.32 & $8,057.38$ & $1,501.91$ & $9,040.55$ & 326.43 & 79.14 & $1,946.95$ \\
\hline Rhode Island & 990,819 & 53.09 & 4.75 & 1.50 & 29.55 & 644.17 & 110.98 & 824.28 & 32.20 & 0.58 & 163.24 \\
\hline South Carolina & $\frac{190,010}{3,885,736}$ & 211.86 & 19.04 & 14.32 & 81.96 & $2,128.64$ & $\frac{11.96}{400.52}$ & $2,697.53$ & $\frac{12.46}{72.16}$ & 13.78 & $\frac{10.24}{514.14}$ \\
\hline South Dakota & 733,133 & 55.97 & 3.74 & 12.68 & 16.10 & 416.91 & 90.29 & 635.99 & 7.92 & 6.78 & 103.96 \\
\hline Tennessee & $5,483,535$ & 316.44 & 28.60 & 34.01 & 75.29 & $3,290.76$ & 632.41 & $4,131.12$ & 92.40 & 20.29 & 796.29 \\
\hline Texas & $20,044,141$ & 953.72 & 92.53 & 54.92 & 375.96 & $12,483.98$ & $2,509.27$ & $14,414.96$ & 374.81 & 564.87 & $3,041.50$ \\
\hline Utah & $2,129,836$ & 111.29 & 9.82 & 20.27 & 49.02 & $1,223.32$ & 233.07 & $1,681.00$ & 27.85 & 62.06 & 276.80 \\
\hline Vermont & 593,740 & 36.73 & 3.44 & 5.62 & 18.87 & 375.49 & 57.01 & 517.29 & 25.02 & 1.73 & 87.68 \\
\hline Virginia & $6,872,912$ & 416.71 & 32.59 & 27.03 & 154.67 & $5,154.87$ & 869.47 & $5,924.64$ & 202.00 & 38.06 & $1,150.47$ \\
\hline Washington & $5,756,361$ & 350.00 & 28.46 & 29.93 & 126.50 & $4,494.62$ & 677.33 & $6,642.06$ & 98.18 & 17.42 & $\begin{array}{c}975.78 \\
975\end{array}$ \\
\hline West Virginia & $1,806,928$ & 96.65 & 8.86 & 9.30 & 26.20 & 798.50 & 140.64 & 947.23 & 23.22 & 111.05 & 214.66 \\
\hline Wisconsin & $5,250,446$ & 311.84 & 25.81 & 68.26 & 108.67 & $3,513.69$ & 646.95 & $4,381.50$ & 104.21 & 8.23 & 813.82 \\
\hline Wyoming & 479,602 & 38.76 & 2.51 & 9.53 & 14.13 & 327.89 & 39.96 & 464.24 & 17.43 & 125.77 & 72.49 \\
\hline $\begin{array}{l}\text { Totals } \\
\end{array}$ & $\overline{\overline{272,690,813}}$ & $15,342.02$ & $1,290.11$ & $1,169.23$ & 6,362.83 & $\overline{189,308.85}$ & $\overline{33,922.37}$ & $\overline{239,671.60}$ & $\begin{array}{l}7,519.38 \\
\end{array}$ & $3,135.15$ & 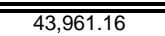 \\
\hline
\end{tabular}


Summary Data: Revenue

\begin{tabular}{|c|c|c|c|c|c|c|c|c|c|}
\hline State & $\begin{array}{l}1999 \text { State } \\
\text { Population }\end{array}$ & $\begin{array}{l}1999 \text { General } \\
\text { Sales Tax } \\
\text { Revenue } \\
\text { (\$ millions) }\end{array}$ & $\begin{array}{l}1999 \text { Motor } \\
\text { Fuels Tax } \\
\text { Revenue } \\
\text { (\$ millions) }\end{array}$ & $\begin{array}{l}1999 \text { Alcohol Tax } \\
\text { Revenue } \\
\text { (\$ millions) }\end{array}$ & $\begin{array}{c}1999 \text { Tobacco } \\
\text { Tax Revenue } \\
\text { (\$ millions) }\end{array}$ & $\begin{array}{c}1999 \text { Insurance } \\
\text { Tax Revenue } \\
\text { (\$ millions) }\end{array}$ & $\begin{array}{c}1999 \text { Public } \\
\text { Utilities Tax } \\
\text { Revenue } \\
\text { (\$ millions) }\end{array}$ & $\begin{array}{l}1999 \text { Pari-Mutuel } \\
\text { Tax Revenue } \\
\text { (\$ millions) }\end{array}$ & $\begin{array}{c}1999 \\
\text { Amusement Tax } \\
\text { Revenue } \\
\text { (\$ millions) }\end{array}$ \\
\hline Alabama & $4,369,862$ & $2,740.49$ & 538.31 & 169.64 & 83.01 & 188.43 & 484.45 & 3.82 & 0.09 \\
\hline Alaska & 619,500 & 126.24 & 38.01 & 12.16 & 51.79 & 28.39 & 3.69 & 0.00 & 2.19 \\
\hline Arizona & $4,778,332$ & $4,294.02$ & 584.75 & 48.15 & 162.84 & 151.88 & 149.22 & 2.95 & 0.72 \\
\hline Arkansas & $2,551,373$ & $2,019.26$ & 380.09 & 29.43 & 94.59 & 68.16 & 75.51 & 6.23 & 0.22 \\
\hline California & $33,145,121$ & $27,736.14$ & $3,034.05$ & 270.99 & 899.89 & $1,263.89$ & $1,995.14$ & 64.08 & 0.00 \\
\hline Colorado & $4,056,133$ & $3,381.21$ & 515.77 & 26.39 & 69.75 & 120.61 & 108.13 & 6.11 & 72.99 \\
\hline Connecticut & $3,282,031$ & $3,218.06$ & 544.67 & 40.76 & 130.54 & 177.63 & 167.70 & 11.03 & 291.03 \\
\hline Delaware & 753,538 & 0.00 & 103.12 & 10.53 & 24.46 & 51.13 & 25.96 & 0.21 & 0.00 \\
\hline District of Columbi & 519,000 & 592.72 & 30.97 & 4.52 & 17.11 & 25.67 & 180.35 & 0.00 & 0.00 \\
\hline $\begin{array}{l}\text { Florida } \\
\text { Fis }\end{array}$ & $15,111,244$ & $14,464.45$ & $2,093.59$ & 577.40 & 453.90 & 336.34 & $1,998.99$ & 50.78 & 0.00 \\
\hline Georgia & $7,788,240$ & $7,113.32$ & 566.40 & 257.79 & 92.50 & 230.46 & 161.86 & 0.00 & 0.00 \\
\hline Hawaii & $1,185,497$ & $1,447.28$ & 134.26 & 37.14 & 42.28 & 54.04 & 149.19 & 0.00 & 0.00 \\
\hline Idaho & $1,251,700$ & 701.66 & 212.49 & 17.06 & 28.49 & 52.32 & 12.61 & 0.00 & 0.00 \\
\hline Illinois & $12,128,370$ & $6,818.07$ & $1,514.05$ & 101.92 & 573.30 & 218.19 & $1,943.17$ & 37.05 & 375.33 \\
\hline Indiana & $5,942,901$ & $3,308.19$ & 663.94 & 35.26 & 89.89 & 157.14 & 6.14 & 3.67 & 405.93 \\
\hline lowa & $2,869,413$ & $1,771.92$ & 340.77 & 17.13 & 98.31 & 114.35 & 14.92 & 3.35 & 153.76 \\
\hline Kansas & $2,654,052$ & $2,153.93$ & 326.00 & 69.76 & 54.55 & 78.26 & 102.65 & 4.12 & 0.98 \\
\hline Kentucky & $3,960,825$ & $2,087.74$ & 444.70 & 21.81 & 17.67 & 212.76 & 147.58 & 26.76 & 0.24 \\
\hline Louisiana & $4,372,035$ & $4,083.59$ & 536.58 & 55.64 & 85.69 & 240.05 & 141.28 & 5.41 & 462.03 \\
\hline Maine & $1,253,040$ & 828.64 & 172.98 & 12.43 & 78.36 & 41.87 & 0.62 & 4.67 & 0.00 \\
\hline Maryland & $5,171,634$ & $2,299.61$ & 680.06 & 24.04 & 128.79 & 178.08 & 293.29 & 0.72 & 10.10 \\
\hline Massachusetts & $6,175,169$ & $3,269.83$ & 636.55 & 61.45 & 284.44 & 336.37 & 0.00 & 8.27 & 6.52 \\
\hline Michigan & $9,863,775$ & $7,230.37$ & $1,074.82$ & 134.15 & 615.45 & 199.46 & 50.92 & 13.29 & 5.99 \\
\hline Minnesota & $4,775,508$ & $3,434.98$ & 580.83 & 60.39 & 184.89 & 179.44 & 52.30 & 0.88 & 62.76 \\
\hline Mississippi & $2,768,619$ & $2,230.31$ & 403.44 & 43.18 & 56.98 & 111.67 & 31.19 & 0.00 & 187.34 \\
\hline Missouri & $5,468,338$ & $\begin{array}{l}3,925.72 \\
\end{array}$ & 665.50 & 24.80 & 130.60 & 216.58 & 331.53 & 0.00 & 164.66 \\
\hline Montana & 882,779 & 0.00 & 160.49 & 16.10 & 14.08 & 38.85 & 16.55 & 0.13 & 37.85 \\
\hline Nebraska & $1,666,028$ & $1,028.50$ & 264.94 & 16.79 & 47.08 & 34.50 & 44.45 & 0.70 & 6.32 \\
\hline Nevada & $1,809,253$ & $1,940.62$ & 300.05 & 17.25 & 64.00 & 116.92 & 73.74 & 0.00 & 633.80 \\
\hline New Hampshire & $1,201,134$ & 0.00 & 119.65 & 11.41 & 73.77 & 57.32 & 56.58 & 3.64 & 1.83 \\
\hline New Jersey & $8,143,412$ & $5,054.44$ & 483.23 & 86.20 & 417.55 & 292.64 & $1,096.47$ & 0.00 & 326.46 \\
\hline New Mexico & $1,739,844$ & $1,812.18$ & 247.19 & 36.56 & 24.75 & 81.39 & 40.90 & 0.70 & 2.89 \\
\hline New York & $18,196,601$ & $15,257.21$ & 505.23 & 195.67 & 696.19 & 716.58 & $2,093.27$ & 36.92 & 0.69 \\
\hline North Carolina & $7,650,789$ & $4,400.28$ & $1,145.97$ & 203.73 & 44.69 & 291.20 & 344.32 & 0.00 & 0.00 \\
\hline North Dakota & 633,666 & 380.25 & 104.89 & 5.33 & 23.03 & 20.98 & 32.15 & 0.00 & 11.15 \\
\hline Ohio & $11,256,654$ & $7,001.95$ & $1,377.97$ & $\begin{array}{l}93.84 \\
\end{array}$ & 298.10 & 356.86 & 696.46 & 16.53 & 0.00 \\
\hline Oklahoma & $3,358,044$ & $2,313.78$ & 384.44 & 61.83 & 77.76 & 143.58 & 91.29 & 4.37 & 8.43 \\
\hline Oregon & $3,316,154$ & 0.00 & 405.54 & 11.82 & 193.81 & 54.13 & 118.21 & 1.20 & 0.11 \\
\hline Pennsylvania & $11,994,016$ & $6,812.20$ & 746.78 & 168.65 & 330.42 & 432.18 & 762.44 & 30.18 & 0.69 \\
\hline Rhode Island & 990,819 & 561.19 & 119.40 & 9.41 & 61.47 & 31.64 & 66.59 & 5.81 & 0.00 \\
\hline South Carolina & $3,885,736$ & $2,423.29$ & 351.00 & 140.52 & 30.15 & 79.72 & 92.94 & 0.00 & 34.76 \\
\hline South Dakota & 733,133 & 563.52 & 107.52 & 10.69 & 20.42 & 35.05 & 2.87 & 1.76 & 0.02 \\
\hline Tennessee & $5,483,535$ & $5,543.11$ & 769.91 & 91.24 & 84.95 & 257.22 & 53.15 & 0.00 & 0.00 \\
\hline Texas & $20,044,141$ & $16,096.65$ & 2,592.32 & 480.47 & 624.07 & 751.17 & $1,174.81$ & 13.34 & 22.96 \\
\hline Utah & $2,129,836$ & $1,763.94$ & 312.71 & 24.23 & 50.46 & 77.66 & 68.78 & 0.00 & 0.00 \\
\hline Vermont & 593,740 & 205.63 & 56.62 & 13.87 & 13.57 & 20.40 & 9.17 & 0.00 & 0.00 \\
\hline Virginia & $6,872,912$ & $3,094.10$ & 794.80 & 113.41 & 50.34 & 244.91 & 526.60 & 0.00 & 0.11 \\
\hline Washington & $5,756,361$ & $8,325.98$ & 718.25 & 159.39 & 278.49 & 238.99 & 553.66 & 1.91 & 0.00 \\
\hline West Virginia & $1,806,928$ & 897.47 & 237.13 & 8.35 & 33.08 & 97.94 & 220.49 & 7.95 & 0.00 \\
\hline Wisconsin & $5,250,446$ & $3,440.14$ & 909.08 & 41.82 & 266.82 & 105.68 & 294.83 & 3.39 & 0.57 \\
\hline Wyoming & 479,602 & 432.63 & 62.62 & 1.16 & 5.79 & 10.04 & 11.26 & 0.29 & 0.00 \\
\hline
\end{tabular}


Summary Data: Revenue

\begin{tabular}{|c|c|c|c|c|c|c|c|c|c|c|c|}
\hline State & $\begin{array}{l}1999 \text { State } \\
\text { Population }\end{array}$ & $\begin{array}{l}1999 \text { Motor } \\
\text { Vehicle License } \\
\text { Tax Revenue } \\
\text { (\$ millions) }\end{array}$ & $\begin{array}{l}1999 \text { Motor } \\
\text { Vehicle Operator } \\
\text { Tax Revenue } \\
\text { (\$ millions) }\end{array}$ & $\begin{array}{l}1999 \text { Hunting and } \\
\text { Fishing License } \\
\text { Tax Revenue } \\
\text { (\$ millions) }\end{array}$ & $\begin{array}{l}1999 \text { Corporate } \\
\text { License Tax } \\
\text { Revenue } \\
\text { ( } \$ \text { millions })\end{array}$ & $\begin{array}{l}1999 \text { Personal } \\
\text { Income Tax } \\
\text { Revenue } \\
\text { (\$ millions) }\end{array}$ & $\begin{array}{l}1999 \text { Corporate } \\
\text { Income Tax } \\
\text { Revenue } \\
\text { (\$ millions) }\end{array}$ & $\begin{array}{c}1999 \text { Property } \\
\text { Tax Revenue } \\
\text { (\$ millions) }\end{array}$ & $\begin{array}{c}1999 \text { Estate and } \\
\text { Gift Tax } \\
\text { Revenue } \\
\text { (\$ millions) }\end{array}$ & $\begin{array}{c}1999 \text { Severance } \\
\text { Tax Revenue } \\
\text { (\$ millions) }\end{array}$ & $\begin{array}{c}1999 \text { Other Tax } \\
\text { Revenue } \\
\text { (\$ millions) } \\
\end{array}$ \\
\hline Alabama & $4,369,862$ & 204.72 & 16.62 & 13.48 & 122.62 & $2,044.78$ & 233.02 & $1,191.79$ & 62.78 & 50.38 & 637.94 \\
\hline Alaska & 619,500 & 43.95 & 0.00 & 21.64 & 1.42 & 0.00 & 211.81 & 727.81 & 1.73 & 426.02 & 67.71 \\
\hline Arizona & $4,778,332$ & 144.06 & 12.96 & 15.28 & 7.11 & $2,098.35$ & 545.39 & $3,584.16$ & 89.09 & 0.00 & 347.26 \\
\hline Arkansas & $2,551,373$ & 106.72 & 7.46 & 19.72 & 11.44 & $1,433.85$ & 212.21 & 966.75 & 32.57 & 10.24 & 167.11 \\
\hline California & $33,145,121$ & $1,662.75$ & 141.11 & 60.01 & 32.82 & $30,732.36$ & $5,459.20$ & $25,424.96$ & 877.90 & 33.74 & $5,271.22$ \\
\hline Colorado & $4,056,133$ & 197.42 & $\frac{171.1}{11.94}$ & 53.51 & 4.94 & $3,354.87$ & 301.04 & $3,413.61$ & 65.39 & 33.98 & 372.85 \\
\hline Connecticut & $3,282,031$ & 225.33 & 29.65 & 4.00 & 12.50 & $3,609.99$ & 474.51 & $5,174.84$ & 250.17 & 0.00 & 522.67 \\
\hline Delaware & 753,538 & 30.29 & 0.14 & 0.76 & 459.86 & 813.72 & 232.53 & 348.52 & 27.06 & 0.00 & 342.34 \\
\hline District of Columbi & 519,000 & 17.93 & 1.51 & 0.00 & 3.68 & 952.16 & 217.60 & 679.55 & 26.25 & 0.00 & 223.29 \\
\hline 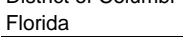 & $15,111,244$ & 910.36 & 113.04 & 26.81 & 126.46 & 0.00 & $1,266.96$ & $13,900.95$ & 649.52 & 67.10 & $3,222.24$ \\
\hline Georgia & $7,788,240$ & 217.90 & 40.31 & 18.88 & 34.59 & $5,696.76$ & 793.17 & $5,422.82$ & 111.19 & 0.00 & 762.72 \\
\hline Hawaii & $1,185,497$ & 106.35 & 0.33 & 0.18 & 2.61 & $1,068.97$ & 52.41 & 594.56 & 28.74 & 0.00 & 193.87 \\
\hline Idaho & $1,251,700$ & 107.81 & 5.67 & 23.54 & 1.16 & 847.02 & 96.15 & 815.66 & 11.13 & 4.55 & 112.84 \\
\hline Illinois & $12,128,370$ & 893.31 & 59.34 & 20.64 & 121.35 & $7,247.52$ & $2,103.93$ & $14,099.97$ & 346.98 & 0.00 & $1,537.16$ \\
\hline Indiana & $5,942,901$ & 141.54 & 0.00 & 14.15 & 4.83 & $4,214.57$ & 989.63 & $5,177.13$ & 148.71 & 0.51 & 219.79 \\
\hline lowa & $2,869,413$ & 325.23 & 10.62 & 18.03 & 32.87 & $1,754.70$ & 234.54 & $2,532.74$ & 72.84 & 0.00 & 180.66 \\
\hline Kansas & $2,654,052$ & 146.96 & 12.03 & 14.99 & 24.82 & $1,696.28$ & 253.84 & $2,115.02$ & 70.24 & 49.28 & 118.71 \\
\hline Kentucky & $3,960,825$ & 211.59 & 6.63 & 16.79 & 173.47 & $3,221.02$ & 312.07 & $1,666.33$ & 81.48 & 174.78 & 888.64 \\
\hline Louisiana & $4,372,035$ & 107.32 & 8.74 & 14.11 & 300.62 & $1,535.65$ & 286.32 & $1,620.13$ & 95.97 & 350.70 & 602.00 \\
\hline Maine & $1,253,040$ & 82.27 & 5.54 & 11.86 & 3.11 & $1,020.01$ & 147.49 & $1,546.86$ & 29.77 & 0.00 & 75.60 \\
\hline Maryland & $5,171,634$ & 177.56 & 21.22 & 9.91 & 11.38 & $6,563.47$ & 404.97 & $4,144.06$ & 126.17 & 0.00 & $1,483.68$ \\
\hline Massachusetts & $6,175,169$ & 234.12 & 59.31 & 6.92 & 21.69 & $8,036.59$ & $1,249.67$ & $7,300.56$ & 173.87 & 0.00 & 584.16 \\
\hline Michigan & $9,863,775$ & 771.70 & 43.57 & 47.67 & 11.99 & $7,475.91$ & $2,413.62$ & $8,810.59$ & 174.89 & 24.21 & 816.04 \\
\hline Minnesota & $4,775,508$ & 596.70 & 27.62 & 44.61 & 4.11 & $5,306.24$ & 779.18 & $4,458.85$ & 58.13 & 2.16 & $1,358.64$ \\
\hline Mississippi & $2,768,619$ & 109.47 & 21.40 & 12.71 & 71.70 & 983.05 & 229.50 & $1,389.92$ & 30.77 & 19.26 & 159.57 \\
\hline Missouri & $5,468,338$ & 264.22 & 21.49 & 29.46 & 102.11 & $3,855.77$ & 276.52 & $3,305.36$ & 118.67 & 0.03 & 597.92 \\
\hline Montana & 882,779 & 63.92 & 5.49 & 28.64 & 1.08 & 483.03 & 89.62 & 891.13 & 18.30 & 74.19 & 101.22 \\
\hline Nebraska & $1,666,028$ & 94.35 & 8.32 & 11.72 & 5.94 & $1,071.88$ & 135.03 & $1,567.01$ & 17.45 & 0.76 & 266.49 \\
\hline Nevada & $1,809,253$ & 111.19 & 11.77 & 6.18 & 19.46 & 0.00 & 0.00 & $1,261.14$ & 41.47 & 33.19 & 661.55 \\
\hline New Hampshire & $1,201,134$ & 61.38 & 8.27 & 6.87 & 4.14 & 63.13 & 255.82 & $2,014.40$ & 49.37 & 0.00 & 322.61 \\
\hline New Jersey & $8,143,412$ & 363.60 & 31.55 & 10.46 & 149.43 & $6,353.98$ & $1,333.96$ & $14,336.03$ & 423.02 & 0.00 & 826.20 \\
\hline New Mexico & $1,739,844$ & 132.61 & 7.40 & 14.88 & 2.29 & 809.57 & 163.96 & 587.85 & 21.91 & 269.94 & 213.99 \\
\hline New York & $18,196,601$ & 702.41 & 84.60 & 32.40 & 73.12 & $26,045.07$ & $5,827.19$ & $24,758.69$ & $1,071.46$ & 0.00 & $4,056.58$ \\
\hline North Carolina & $7,650,789$ & 425.46 & 81.80 & 21.04 & 227.00 & $6,586.15$ & 920.58 & $4,350.64$ & 182.85 & 1.95 & $1,050.48$ \\
\hline North Dakota & 633,666 & 39.83 & 3.31 & 6.62 & 0.00 & 181.98 & 93.60 & 497.22 & 7.42 & 100.11 & 155.72 \\
\hline Ohio & $11,256,654$ & 685.40 & 39.22 & 31.83 & 394.45 & $10,288.39$ & 751.57 & $9,334.35$ & 141.46 & 12.17 & 786.29 \\
\hline Oklahoma & $3,358,044$ & 579.53 & 6.84 & 15.02 & 38.53 & $2,070.45$ & 187.31 & $1,237.65$ & 88.80 & 210.21 & 252.43 \\
\hline Oregon & $3,316,154$ & 340.83 & 20.44 & 30.71 & 5.98 & $3,709.59$ & 324.39 & $2,558.19$ & 47.98 & 49.56 & 664.91 \\
\hline Pennsylvania & $11,994,016$ & 735.84 & 51.86 & 45.80 & $1,116.33$ & $8,846.07$ & $1,537.72$ & $9,659.06$ & 760.70 & 0.00 & $3,155.49$ \\
\hline Rhode Island & $\begin{array}{c}990,819 \\
\end{array}$ & 52.58 & 0.41 & 0.72 & 12.22 & 762.78 & 66.32 & $1,285.11$ & 46.85 & 0.00 & 114.04 \\
\hline South Carolina & $3,885,736$ & 113.04 & 15.03 & 12.32 & 37.16 & $2,298.23$ & 257.49 & $2,475.95$ & 57.19 & 0.00 & 662.51 \\
\hline South Dakota & 733,133 & 48.93 & 1.35 & 14.25 & 1.89 & 0.00 & 50.82 & 617.29 & 26.43 & 3.18 & 145.41 \\
\hline Tennessee & $5,483,535$ & 297.35 & 36.72 & 135.66 & 323.38 & 160.18 & 571.43 & $2,684.03$ & 89.13 & 0.80 & 692.04 \\
\hline Texas & $20,044,141$ & $1,136.96$ & 98.55 & 56.44 & $2,127.76$ & 0.00 & 0.00 & $18,804.96$ & 256.28 & 705.25 & $4,281.04$ \\
\hline Utah & $2,129,836$ & 78.15 & 5.79 & 19.37 & 1.52 & $1,461.30$ & 180.14 & $1,191.69$ & 8.24 & 14.51 & 213.39 \\
\hline Vermont & 593,740 & 33.65 & 3.75 & 6.26 & 1.27 & 383.45 & 49.70 & 765.69 & 23.36 & 0.00 & 198.76 \\
\hline Virginia & $6,872,912$ & 399.60 & 27.58 & 20.29 & 27.53 & $6,087.86$ & 414.39 & $5,757.55$ & 154.08 & 1.82 & $1,840.19$ \\
\hline Washington & $5,756,361$ & 393.19 & 27.65 & 25.81 & 13.17 & 0.00 & 0.00 & $5,763.41$ & 69.70 & 62.32 & $1,483.37$ \\
\hline West Virginia & $1,806,928$ & 81.68 & 4.47 & 15.36 & 7.90 & 919.95 & 263.12 & 811.77 & 27.33 & 153.36 & 486.90 \\
\hline Wisconsin & $5,250,446$ & 276.68 & 27.33 & 58.84 & 59.92 & $5,162.24$ & 670.96 & $5,524.61$ & 116.90 & 2.46 & 440.95 \\
\hline Wyoming & 479,602 & 56.33 & 2.38 & 22.09 & 6.10 & 0.00 & 0.00 & 522.70 & 9.73 & 192.44 & 21.98 \\
\hline Totals & $272,690,813$ & $\overline{15,342.02}$ & $\overline{1,290.11}$ & 11,169.23 & "6,362.83 & $=189,308.85$ & 33,922.37 & $239,671.60$ & $7,519.38$ & "3,135.15 & "43,961.16 \\
\hline
\end{tabular}




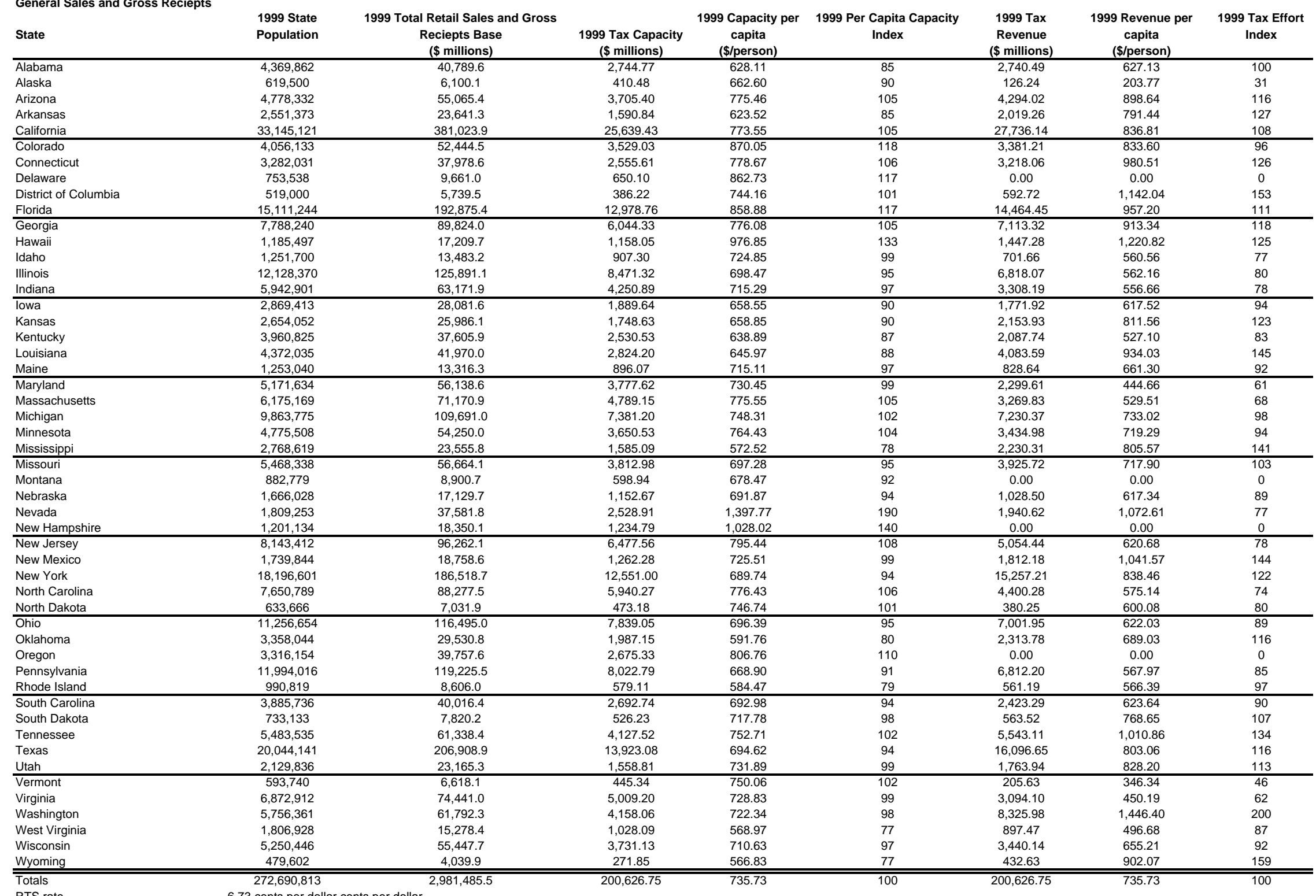


Selective Sales--Total

\begin{tabular}{|c|c|c|c|c|c|c|c|c|}
\hline State & $\begin{array}{l}1999 \text { State } \\
\text { Population }\end{array}$ & 1999 Tax Base & $\begin{array}{c}1999 \text { Tax Capacity } \\
\text { (\$ millions) }\end{array}$ & $\begin{array}{l}1999 \text { Capacity } \\
\text { per capita } \\
\text { (\$/person) }\end{array}$ & $\begin{array}{l}1999 \text { Per Capita } \\
\text { Capacity Index }\end{array}$ & $\begin{array}{l}1999 \text { Tax Revenue } \\
\text { (\$ millions) }\end{array}$ & $\begin{array}{l}1999 \text { Revenue } \\
\text { per capita } \\
\text { (\$/person) }\end{array}$ & $\begin{array}{l}1999 \text { Tax Effort } \\
\text { Index }\end{array}$ \\
\hline Alabama & $4,369,862$ & $n / a$ & $1,235.7$ & 282.77 & 105 & $1,447.84$ & 331.32 & 117 \\
\hline Alaska & 619,500 & $\mathrm{n} / \mathrm{a}$ & 162.9 & 262.91 & 98 & 137.16 & 221.40 & 84 \\
\hline Arizona & $4,778,332$ & $\mathrm{n} / \mathrm{a}$ & $1,289.2$ & 269.80 & 101 & $1,100.51$ & 230.31 & 85 \\
\hline Arkansas & $2,551,373$ & $\mathrm{n} / \mathrm{a}$ & 747.4 & 292.93 & 109 & 653.84 & 256.27 & 87 \\
\hline California & $33,145,121$ & $n / a$ & $7,545.7$ & 227.66 & 85 & $7,530.44$ & 227.20 & 100 \\
\hline Colorado & $4,056,133$ & $n / a$ & $1,142.1$ & 281.57 & 105 & 919.68 & 226.74 & 81 \\
\hline Connecticut & $3,282,031$ & $\mathrm{n} / \mathrm{a}$ & 996.2 & 303.53 & 113 & $1,366.74$ & 416.43 & 137 \\
\hline Delaware & 753,538 & $\mathrm{n} / \mathrm{a}$ & 274.1 & 363.70 & 136 & 216.03 & 286.68 & 79 \\
\hline D.C. & 519,000 & $\mathrm{n} / \mathrm{a}$ & 180.6 & 347.91 & 130 & 258.91 & 498.86 & 143 \\
\hline Florida & $15,111,244$ & $\mathrm{n} / \mathrm{a}$ & $4,288.8$ & 283.81 & 106 & $5,510.46$ & 364.66 & 128 \\
\hline Georgia & $7,788,240$ & $\mathrm{n} / \mathrm{a}$ & $2,313.5$ & 297.05 & 111 & $1,288.71$ & 165.47 & 56 \\
\hline Hawaii & $1,185,497$ & $\mathrm{n} / \mathrm{a}$ & 237.6 & 200.46 & 75 & 418.27 & 352.82 & 176 \\
\hline Idaho & $1,251,700$ & $\mathrm{n} / \mathrm{a}$ & 330.8 & 264.26 & 99 & 311.67 & 249.00 & 94 \\
\hline Illinois & $12,128,370$ & $\mathrm{n} / \mathrm{a}$ & $3,148.0$ & 259.56 & 97 & $4,718.33$ & 389.03 & 150 \\
\hline Indiana & $5,942,901$ & $\mathrm{n} / \mathrm{a}$ & $1,809.6$ & 304.49 & 114 & $1,357.28$ & 228.39 & 75 \\
\hline lowa & $2,869,413$ & $n / a$ & 846.5 & 295.01 & 110 & 737.36 & 256.97 & 87 \\
\hline Kansas & $2,654,052$ & $\mathrm{n} / \mathrm{a}$ & 754.1 & 284.12 & 106 & 636.84 & 239.95 & 84 \\
\hline Kentucky & $3,960,825$ & $\mathrm{n} / \mathrm{a}$ & $1,198.9$ & 302.70 & 113 & 918.85 & 231.98 & 77 \\
\hline Louisiana & $4,372,035$ & $\mathrm{n} / \mathrm{a}$ & $1,272.4$ & 291.03 & 109 & $1,523.56$ & 348.48 & 120 \\
\hline Maine & $1,253,040$ & $\mathrm{n} / \mathrm{a}$ & 338.6 & 270.23 & 101 & 331.14 & 264.27 & 98 \\
\hline Maryland & $5,171,634$ & $\mathrm{n} / \mathrm{a}$ & $1,319.6$ & 255.16 & 95 & $1,314.94$ & 254.26 & 100 \\
\hline Massachusetts & $6,175,169$ & $\mathrm{n} / \mathrm{a}$ & $1,633.6$ & 264.55 & 99 & $1,333.67$ & 215.97 & 82 \\
\hline Michigan & $9,863,775$ & $\mathrm{n} / \mathrm{a}$ & $2,624.8$ & 266.10 & 99 & $2,086.51$ & 211.53 & 79 \\
\hline Minnesota & $4,775,508$ & $\mathrm{n} / \mathrm{a}$ & $1,307.2$ & 273.73 & 102 & $1,119.50$ & 234.42 & 86 \\
\hline Mississippi & $2,768,619$ & $\mathrm{n} / \mathrm{a}$ & 840.1 & 303.42 & 113 & 830.40 & 299.93 & 99 \\
\hline Missouri & $5,468,338$ & $\mathrm{n} / \mathrm{a}$ & $1,661.2$ & 303.78 & 113 & $1,533.66$ & 280.46 & 92 \\
\hline Montana & 882,779 & $\mathrm{n} / \mathrm{a}$ & 248.9 & 281.98 & 105 & 284.07 & 321.79 & 114 \\
\hline Nebraska & $1,666,028$ & $\mathrm{n} / \mathrm{a}$ & 505.8 & 303.60 & 113 & 415.23 & 249.23 & 82 \\
\hline Nevada & $1,809,253$ & $\mathrm{n} / \mathrm{a}$ & 570.5 & 315.30 & 118 & $1,203.85$ & 665.38 & 211 \\
\hline New Hampshire & $1,201,134$ & $\mathrm{n} / \mathrm{a}$ & 404.8 & 337.02 & 126 & 324.35 & 270.03 & 80 \\
\hline New Jersey & $8,143,412$ & $\mathrm{n} / \mathrm{a}$ & $2,321.5$ & 285.08 & 106 & $2,692.33$ & 330.61 & 116 \\
\hline New Mexico & $1,739,844$ & $\mathrm{n} / \mathrm{a}$ & 485.3 & 278.94 & 104 & 433.38 & 249.09 & 89 \\
\hline New York & $18,196,601$ & $\mathrm{n} / \mathrm{a}$ & $4,217.8$ & 231.79 & 86 & $4,251.83$ & 233.66 & 101 \\
\hline North Carolina & $7,650,789$ & $\mathrm{n} / \mathrm{a}$ & $2,216.8$ & 289.75 & 108 & $2,023.35$ & 264.46 & 91 \\
\hline North Dakota & 633,666 & $\mathrm{n} / \mathrm{a}$ & 196.1 & 309.39 & 115 & 198.05 & 312.54 & 101 \\
\hline Ohio & $11,256,654$ & $\mathrm{n} / \mathrm{a}$ & $3,056.5$ & 271.53 & 101 & $2,839.09$ & 252.21 & 93 \\
\hline Oklahoma & $3,358,044$ & $\mathrm{n} / \mathrm{a}$ & 955.0 & 284.40 & 106 & 769.08 & 229.03 & 81 \\
\hline Oregon & $3,316,154$ & $\mathrm{n} / \mathrm{a}$ & 796.7 & 240.24 & 90 & 785.33 & 236.82 & 99 \\
\hline Pennsylvania & $11,994,016$ & $\mathrm{n} / \mathrm{a}$ & $3,051.0$ & 254.38 & 95 & $2,473.19$ & 206.20 & 81 \\
\hline Rhode Island & 990,819 & $\mathrm{n} / \mathrm{a}$ & 242.0 & 244.24 & 91 & 294.22 & 296.95 & 122 \\
\hline South Carolina & $3,885,736$ & $\mathrm{n} / \mathrm{a}$ & $1,178.5$ & 303.29 & 113 & 713.50 & 183.62 & 61 \\
\hline South Dakota & 733,133 & $\mathrm{n} / \mathrm{a}$ & 221.3 & 301.84 & 113 & 178.45 & 243.40 & 81 \\
\hline Tennessee & $5,483,535$ & $\mathrm{n} / \mathrm{a}$ & $1,584.6$ & 288.97 & 108 & $1,330.75$ & 242.68 & 84 \\
\hline Texas & $20,044,141$ & $\mathrm{n} / \mathrm{a}$ & $5,337.7$ & 266.30 & 99 & $5,661.84$ & 282.47 & 106 \\
\hline Utah & $2,129,836$ & $n / a$ & 482.4 & 226.51 & 84 & 532.22 & 249.89 & 110 \\
\hline Vermont & 593,740 & $n / a$ & 175.7 & 295.86 & 110 & 113.79 & 191.64 & 65 \\
\hline Virginia & $6,872,912$ & $\mathrm{n} / \mathrm{a}$ & $1,915.0$ & 278.63 & 104 & $1,732.97$ & 252.14 & 90 \\
\hline Washington & $5,756,361$ & $\mathrm{n} / \mathrm{a}$ & $1,365.5$ & 237.22 & 88 & $1,951.04$ & 338.94 & 143 \\
\hline West Virginia & $1,806,928$ & $\mathrm{n} / \mathrm{a}$ & 472.2 & 261.33 & 97 & 607.37 & 336.13 & 129 \\
\hline Wisconsin & $5,250,446$ & $\mathrm{n} / \mathrm{a}$ & $1,430.0$ & 272.35 & 102 & $1,622.46$ & 309.01 & 113 \\
\hline Wyoming & 479,602 & $\mathrm{n} / \mathrm{a}$ & 200.9 & 418.94 & 156 & 91.27 & 190.29 & 45 \\
\hline $\begin{array}{ll}\text { Totals } \\
\end{array}$ & $\overline{272,690,813}$ & $\overline{n / a}$ & $73,117.7$ & 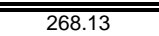 & 100 & 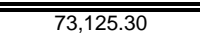 & 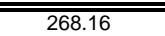 & 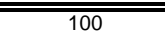 \\
\hline
\end{tabular}




\begin{tabular}{|c|c|c|c|c|c|c|c|c|}
\hline State & Population & $\begin{array}{l}1999 \text { Base: Motor Fuel } \\
\text { (millions of gallons) }\end{array}$ & $\begin{array}{l}1999 \text { Tax Capacity } \\
\text { (\$ millions) }\end{array}$ & $\begin{array}{c}1999 \text { Capacity per capita } \\
\text { (\$/person) }\end{array}$ & $\begin{array}{l}1999 \text { Per Capita } \\
\text { Capacity Index }\end{array}$ & $\begin{array}{l}1999 \text { Tax Revenue } \\
\text { (\$ millions) }\end{array}$ & $\begin{array}{l}1999 \text { Revenue per capita } \\
\text { (\$/person) }\end{array}$ & 1999 Tax Effort Index \\
\hline Alabama & $4,369,862$ & $3,134.3$ & 593.6 & 135.85 & 123 & 538.31 & 123.19 & 91 \\
\hline Alaska & 619,500 & 360.4 & 68.3 & 110.19 & 100 & 38.01 & 61.36 & 56 \\
\hline Arizona & $4,778,332$ & $2,891.5$ & 547.6 & 114.61 & 104 & 584.75 & 122.37 & 107 \\
\hline Arkansas & $2,551,373$ & $1,965.1$ & 372.2 & 145.88 & 132 & 380.09 & 148.97 & 102 \\
\hline California & $33,145,121$ & $16,618.6$ & $3,147.6$ & 94.96 & 86 & $3,034.05$ & 91.54 & 96 \\
\hline Colorado & $4,056,133$ & $2,308.7$ & 437.3 & 107.81 & 98 & 515.77 & 127.16 & 118 \\
\hline Connecticut & $3,282,031$ & $1,611.8$ & 305.3 & 93.02 & 84 & 544.67 & 165.95 & 178 \\
\hline Delaware & 753,538 & 444.9 & 84.3 & 111.84 & 101 & 103.12 & 136.85 & 122 \\
\hline D.C. & 519,000 & 166.6 & 31.6 & 60.80 & 55 & 30.97 & 59.66 & 98 \\
\hline Florida & $15,111,244$ & $8,519.1$ & $1,613.5$ & 106.78 & 97 & $2,093.59$ & 138.55 & 130 \\
\hline Georgia & $7,788,240$ & $5,896.3$ & $1,116.8$ & 143.39 & 130 & 566.40 & 72.73 & 51 \\
\hline Hawaii & $1,185,497$ & 422.9 & 80.1 & 67.57 & 61 & 134.26 & 113.25 & 168 \\
\hline Idaho & $1,251,700$ & 850.2 & 161.0 & 128.65 & 117 & 212.49 & 169.76 & 132 \\
\hline Illinois & $12,128,370$ & $6,128.1$ & $1,160.7$ & 95.70 & 87 & $1,514.05$ & 124.84 & 130 \\
\hline Indiana & $5,942,901$ & $4,061.0$ & 769.2 & 129.42 & 117 & 663.94 & 111.72 & 86 \\
\hline lowa & $2,869,413$ & $1,996.4$ & 378.1 & 131.78 & 119 & 340.77 & 118.76 & 90 \\
\hline Kansas & $2,654,052$ & $1,735.1$ & 328.6 & 123.82 & 112 & 326.00 & 122.83 & 99 \\
\hline Kentucky & $3,960,825$ & $2,835.2$ & 537.0 & 135.58 & 123 & 444.70 & 112.27 & 83 \\
\hline Louisiana & $4,372,035$ & $2,687.6$ & 509.0 & 116.43 & 105 & 536.58 & 122.73 & 105 \\
\hline Maine & $1,253,040$ & 808.7 & 153.2 & 122.24 & 111 & 172.98 & 138.04 & 113 \\
\hline Maryland & $5,171,634$ & $2,818.2$ & 533.8 & 103.21 & 94 & 680.06 & 131.50 & 127 \\
\hline Massachusetts & $6,175,169$ & $3,039.5$ & 575.7 & 93.23 & 84 & 636.55 & 103.08 & 111 \\
\hline Michigan & $9,863,775$ & $5,839.9$ & $1,106.1$ & 112.14 & 102 & $1,074.82$ & 108.97 & 97 \\
\hline Minnesota & $4,775,508$ & $2,953.1$ & 559.3 & 117.12 & 106 & 580.83 & 121.63 & 104 \\
\hline Mississippi & $2,768,619$ & $2,105.1$ & 398.7 & 144.01 & 130 & 403.44 & 145.72 & 101 \\
\hline Missouri & $5,468,338$ & $3,915.3$ & 741.6 & 135.61 & 123 & 665.50 & 121.70 & 90 \\
\hline Montana & 882,779 & 666.0 & 126.1 & 142.90 & 129 & 160.49 & 181.80 & 127 \\
\hline Nebraska & $1,666,028$ & $1,227.9$ & 232.6 & 139.59 & 126 & 264.94 & 159.03 & 114 \\
\hline Nevada & $\begin{array}{l}1,809,253 \\
1\end{array}$ & $1,141.0$ & 216.1 & 119.45 & 108 & 300.05 & 165.84 & 139 \\
\hline New Hampshire & $1,201,134$ & 749.5 & 142.0 & 118.19 & 107 & 119.65 & 99.62 & 84 \\
\hline New Jersey & $8,143,412$ & $4,647.7$ & 880.3 & 108.10 & 98 & 483.23 & 59.34 & 55 \\
\hline New Mexico & $1,739,844$ & $1,317.5$ & 249.5 & 143.42 & 130 & 247.19 & 142.07 & 99 \\
\hline New York & $18,196,601$ & $6,549.7$ & $1,240.5$ & 68.17 & 62 & 505.23 & 27.77 & 41 \\
\hline North Carolina & $7,650,789$ & $4,884.7$ & 925.2 & 120.93 & 110 & $1,145.97$ & 149.78 & 124 \\
\hline North Dakota & 633,666 & 512.1 & 97.0 & 153.06 & 139 & $\begin{array}{l}104.89 \\
109\end{array}$ & 165.53 & 108 \\
\hline Ohio & $11,256,654$ & $6,446.9$ & $1,221.1$ & 108.48 & 98 & $1,377.97$ & 122.41 & 113 \\
\hline Oklahoma & $3,358,044$ & $2,444.8$ & 463.1 & 137.90 & 125 & 384.44 & 114.48 & 83 \\
\hline Oregon & $3,316,154$ & $1,649.2$ & 312.4 & 94.19 & 85 & 405.54 & 122.29 & 130 \\
\hline Pennsylvania & $11,994,016$ & $6,220.5$ & $1,178.2$ & 98.23 & 89 & 746.78 & 62.26 & 63 \\
\hline Rhode Island & 990,819 & 455.3 & 86.2 & 87.02 & 79 & 119.40 & 120.51 & 138 \\
\hline South Carolina & $3,885,736$ & $2,785.5$ & 527.6 & 135.77 & 123 & 351.00 & 90.33 & 67 \\
\hline South Dakota & 733,133 & 550.9 & 104.4 & 142.33 & 129 & 107.52 & 146.66 & 103 \\
\hline Tennessee & $5,483,535$ & $3,703.9$ & 701.5 & 127.93 & 116 & 769.91 & 140.40 & 110 \\
\hline Texas & $20,044,141$ & $12,694.4$ & $2,404.3$ & 119.95 & 109 & $2,592.32$ & 129.33 & 108 \\
\hline Utah & $2,129,836$ & $1,265.8$ & 239.8 & 112.57 & 102 & 312.71 & 146.82 & 130 \\
\hline Vermont & 593,740 & 391.4 & 74.1 & 124.85 & 113 & 56.62 & 95.36 & 76 \\
\hline Virginia & $6,872,912$ & $4,423.8$ & 837.9 & 121.91 & 110 & 794.80 & 115.64 & 95 \\
\hline Washington & $5,756,361$ & $3,130.7$ & 593.0 & 103.01 & 93 & 718.25 & 124.77 & 121 \\
\hline West Virginia & $1,806,928$ & $1,109.3$ & 210.1 & 116.28 & 105 & 237.13 & 131.23 & 113 \\
\hline Wisconsin & $5,250,446$ & $3,169.8$ & 600.4 & 114.34 & 104 & 909.08 & 173.14 & 151 \\
\hline Wyoming & 479,602 & 639.3 & 121.1 & 252.49 & 229 & 62.62 & 130.56 & 52 \\
\hline $\begin{array}{l}\text { Totals } \\
\end{array}$ & $\overline{272,690,813}$ & $158,891.4$ & 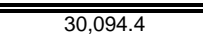 & 1110.36 & 100 & 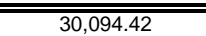 & 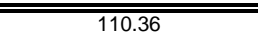 & 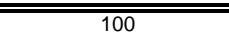 \\
\hline
\end{tabular}


Selective Sales--Alcoholic Beverages

\begin{tabular}{|c|c|c|c|c|c|c|c|c|}
\hline State & $\begin{array}{l}1999 \text { State } \\
\text { Population }\end{array}$ & $\begin{array}{c}1999 \text { Base:Total } \\
\text { Alcohol } \\
\text { (millions) }\end{array}$ & $\begin{array}{l}1999 \text { Tax Capacity } \\
\text { (\$ millions) }\end{array}$ & $\begin{array}{c}1999 \text { Capacity per } \\
\text { capita } \\
\text { (\$/person) }\end{array}$ & $\begin{array}{l}1999 \text { Per Capita } \\
\text { Capacity Index }\end{array}$ & $\begin{array}{l}1999 \text { Tax Revenue } \\
\text { (\$ millions) }\end{array}$ & $\begin{array}{c}1999 \text { Revenue per } \\
\text { capita } \\
\text { (\$/person) }\end{array}$ & $\begin{array}{l}1999 \text { Tax Effort } \\
\text { Index }\end{array}$ \\
\hline Alabama & $4,369,862$ & 6.6 & 57.4 & 13.14 & 86 & 169.6 & 38.82 & 295 \\
\hline Alaska & 619,500 & 1.3 & 11.5 & 18.63 & 121 & 12.2 & 19.62 & 105 \\
\hline Arizona & $4,778,332$ & 9.7 & 85.0 & 17.80 & 116 & 48.2 & 10.08 & 57 \\
\hline Arkansas & $2,551,373$ & 3.7 & 32.3 & 12.65 & 82 & 29.4 & 11.54 & 91 \\
\hline California & $33,145,121$ & 56.5 & 494.9 & 14.93 & 97 & 271.0 & 8.18 & 55 \\
\hline Colorado & $4,056,133$ & 8.3 & 72.9 & 17.98 & 117 & 26.4 & 6.51 & 36 \\
\hline Connecticut & $3,282,031$ & 5.9 & 52.0 & 15.84 & 103 & 40.8 & 12.42 & 78 \\
\hline Delaware & 753,538 & 1.8 & 15.5 & 20.61 & 134 & 10.5 & 13.98 & 68 \\
\hline D.C. & 519,000 & 1.7 & 14.6 & 28.06 & 183 & 4.5 & 8.71 & 31 \\
\hline Florida & $15,111,244$ & 32.1 & 281.0 & 18.59 & 121 & 577.4 & 38.21 & 205 \\
\hline Georgia & $7,788,240$ & 13.7 & 120.1 & 15.42 & 100 & 257.8 & 33.10 & 215 \\
\hline Hawaii & $1,185,497$ & 2.2 & 19.3 & 16.24 & 106 & 37.1 & 31.33 & 193 \\
\hline Idaho & $1,251,700$ & 2.3 & 20.0 & 15.96 & 104 & 17.1 & 13.63 & 85 \\
\hline Illinois & $12,128,370$ & 22.2 & 194.2 & 16.01 & 104 & 101.9 & 8.40 & 52 \\
\hline Indiana & $5,942,901$ & 9.3 & 81.4 & 13.71 & 89 & 35.3 & 5.93 & 43 \\
\hline lowa & $2,869,413$ & 4.5 & 39.7 & 13.83 & 90 & 17.1 & 5.97 & 43 \\
\hline Kansas & $2,654,052$ & 3.9 & 33.8 & 12.73 & 83 & 69.8 & 26.28 & 206 \\
\hline Kentucky & $3,960,825$ & 5.6 & 49.1 & 12.40 & 81 & 21.8 & 5.51 & 44 \\
\hline Louisiana & $4,372,035$ & 8.6 & 75.3 & 17.23 & 112 & 55.6 & 12.73 & 74 \\
\hline Maine & $1,253,040$ & 2.3 & 20.0 & 15.98 & 104 & 12.4 & 9.92 & 62 \\
\hline Maryland & $5,171,634$ & 8.7 & 76.1 & 14.72 & 96 & 24.0 & 4.65 & 32 \\
\hline Massachusetts & $6,175,169$ & 12.2 & 107.2 & 17.36 & 113 & 61.4 & 9.95 & 57 \\
\hline Michigan & $9,863,775$ & 16.6 & 145.5 & 14.75 & 96 & 134.1 & 13.60 & 92 \\
\hline Minnesota & $4,775,508$ & 9.1 & 80.1 & 16.77 & 109 & 60.4 & 12.65 & 75 \\
\hline Mississippi & $2,768,619$ & 4.7 & 41.2 & 14.89 & 97 & 43.2 & 15.59 & 105 \\
\hline Missouri & $5,468,338$ & 9.8 & 85.9 & 15.72 & 102 & 24.8 & 4.53 & 29 \\
\hline Montana & 882,779 & 1.8 & 15.7 & 17.78 & 116 & 16.1 & 18.24 & 103 \\
\hline Nebraska & $1,666,028$ & 3.0 & 25.9 & 15.52 & 101 & 16.8 & 10.08 & 65 \\
\hline Nevada & $1,809,253$ & 5.6 & 48.7 & 26.93 & 176 & 17.3 & 9.54 & 35 \\
\hline New Hampshire & $1,201,134$ & 3.9 & 34.2 & 28.43 & 185 & 11.4 & 9.50 & 33 \\
\hline New Jersey & $8,143,412$ & 14.3 & 125.1 & 15.37 & 100 & 86.2 & 10.59 & 69 \\
\hline New Mexico & $1,739,844$ & 3.3 & 28.5 & 16.39 & 107 & 36.6 & 21.01 & 128 \\
\hline New York & $18,196,601$ & 28.2 & 246.8 & 13.56 & 88 & 195.7 & 10.75 & 79 \\
\hline North Carolina & $7,650,789$ & 12.0 & 105.4 & 13.77 & 90 & 203.7 & 26.63 & 193 \\
\hline North Dakota & 633,666 & 1.3 & 11.0 & 17.37 & 113 & 5.3 & 8.42 & 48 \\
\hline Ohio & $11,256,654$ & 18.1 & 158.2 & 14.05 & 92 & 93.8 & 8.34 & 59 \\
\hline Oklahoma & $3,358,044$ & 4.6 & 40.2 & 11.98 & 78 & 61.8 & 18.41 & 154 \\
\hline Oregon & $3,316,154$ & 6.2 & 54.0 & 16.30 & 106 & 11.8 & 3.57 & 22 \\
\hline Pennsylvania & $11,994,016$ & 18.6 & 163.4 & 13.63 & 89 & 168.7 & 14.06 & 103 \\
\hline Rhode Island & 990,819 & 1.9 & 16.8 & 16.91 & 110 & 9.4 & 9.50 & 56 \\
\hline South Carolina & $3,885,736$ & 7.5 & 65.6 & 16.89 & 110 & 140.5 & 36.16 & 214 \\
\hline South Dakota & 733,133 & 1.3 & 11.7 & 16.01 & 104 & 10.7 & 14.59 & 91 \\
\hline Tennessee & $5,483,535$ & 8.4 & 73.4 & 13.38 & 87 & 91.2 & 16.64 & 124 \\
\hline Texas & $20,044,141$ & 35.4 & 310.7 & 15.50 & 101 & 480.5 & 23.97 & 155 \\
\hline Utah & $2,129,836$ & 2.0 & 17.8 & $\begin{array}{l}8.34 \\
\end{array}$ & 54 & 24.2 & 11.38 & 136 \\
\hline Vermont & 593,740 & 1.1 & 10.0 & 16.86 & 110 & 13.9 & 23.36 & 139 \\
\hline Virginia & $6,872,912$ & 11.0 & 96.0 & 13.96 & 91 & 113.4 & 16.50 & 118 \\
\hline Washington & $5,756,361$ & 10.0 & 87.7 & 15.24 & 99 & 159.4 & 27.69 & 182 \\
\hline West Virginia & $1,806,928$ & 2.5 & 21.7 & 12.00 & 78 & 8.4 & 4.62 & 39 \\
\hline Wisconsin & $5,250,446$ & 11.5 & 100.6 & 19.16 & 125 & 41.8 & 7.97 & 42 \\
\hline Wyoming & 479,602 & 1.0 & 8.3 & 17.39 & 113 & 1.2 & 2.42 & 14 \\
\hline Totals & $\overline{272,690,813}$ & $\overline{4777.4}$ & $4,183.7$ & 15.34 & 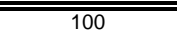 & $4,183.7$ & 15.34 & $\overline{100}$ \\
\hline
\end{tabular}


1999 State Population 1999 Base:Distilled Spirits Consumption 1999 Tax Capacity

Alabama

Alaska

Arizona
Arkansas

California

Colorado

Connecticut

D.C.

Florida

Georgia

Hawail

Idaho

Illinois

Indian

owa

Kentucky

Louisiana

Maine

Maryland
Massachusetts

Michigan

Minnesota
Mississippi

Missouri

Montana

Nebraska

New Hampshir

New Jersey

rex

North Carolina

North Dakota

Ohio

Oklahoma

Oregon

Pennsylvania

South Carolina

South Carolina
South Dakota

South Dakota

Texas

Utah

Vermont

Washington

West Virginia

Wisconsin

Wyoming (millions of gallons)

$4,369,862$
619,500

619,500
$4,778,332$

2,551,373

43,145,121

$3,282,031$

753,538

$5,111,244$

$7,788,240$

$1,185,497$

$1,251,700$

$12,128,370$

(2,869,413

4,372,035

$1,253,040$

$5,171,634$
$6,175,169$

$9,863,775$

$9,863,775$

$2,768,619$

$5,468,338$

882,779

$1,809,25$

$1,201,134$

$8,143,412$

$18,196,601$

$7,650,789$

$11,256,654$

$3,358,044$

$3,316,154$

$11,994,016$

$11,994,016$
990,819

$3,885,736$

$3,885,736$
733,133

$5,483,535$

$2,129,836$

593,740
$6,872,912$

$5,756,36$

$1,806,928$

$5,250,446$

$272,690,813$

$\$ 13.65 /$ gallo

0.46
2.69

2.69
1.11
16.01

16.01
2.64

2.02

0.62

10.22
4.39

4.39
0.54

0.51

6.35
2.89

2.89
1.11

1.15

1.73
2.44
0.73

0.73

3.07
3.95

5.40

1.34

2.86
0.48

0.80

0.80
1.91
1.67
4.69

4.69

0.82
8.43

3.24

0.40
4.32

4.32
1.20

1.69
4.58

0.56

2.29

0.41
2.24
7.79

2.24
7.79
0.62

0.62

2.65

2.93

3.66

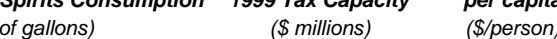

\begin{tabular}{cc}
0.33 & 4.5 \\
\hline 138.51 & $1,891.8$
\end{tabular}

6.94

Capacity Index

147.62
110.73

85.73

95.08
127.94
121.14

162.77

251.88
133.14

133.14

89.34

79.66

103.12

95.61
76.47

76.47
85.12

85.12
85.74

110.05

114.54

116.68

125.92
107.78

134.68

94.93

102.84
106.16

106.16
94.71

207.89

274.05

113.37
92.33

91.25

83.32
125.36

75.61

70.26
100.60

100.60
75.10

75.10
110.77

115.90
110.64

80.55

76.49
56.94

56.94
100.80

100.80
75.92

100.19

60.58

60.58
137.05
133.82

100.00

1999 Tax Revenu

1999 Revenue per

capita

1999 Tax Effort

64.16
6.09

(\$/person)

Index

259
96

$\begin{array}{lll}19.65 & 4.11 & 54 \\ 11.17 & 4.38 & 74\end{array}$

$\begin{array}{lll}127.52 & 3.85 & 58\end{array}$

$15.43-3.80$

$\begin{array}{lll}22.43 & 6.84 & 81\end{array}$

$\begin{array}{lll}5.43 & 7.21 & 64 \\ 2.38 & 4.58 & 26 \\ 2.38 & 14.22 & 153\end{array}$

$\begin{array}{lll}213.22 & 14.11 & 153\end{array}$

$\begin{array}{lrr}59.43 & 7.63 & 99 \\ 7.77 & 6.56 & 106 \\ 11.01 & 8.80 & 159\end{array}$

$\begin{array}{lll}71.01 & 8.50 & 159\end{array}$

52.88

17.51

0.00
24.30

13.94

14.36

1.49
11.16

36.81

86.34
41.17

11.39
14.07

14.07
10.90

5.74

9.05
0.04

51.80

12.24
126.73

112.12
2.48

2.48
34.79

29.24
0.00

0.00
103.38

4.96

59.29

4.54

47.34
168.35

168.35
10.93

10.93
8.98
53.29

53.29
113.37

0.00

30.41
0.78

8.80
4.36

4.36
2.95

0.00
9.16

3.52

3.29
1.19

1.19
2.16
5.96

5.96
8.75
8.62

8.62

2.57
12.35
3.45

12.35
3.45

3.45
0.03

0.03
6.36
7.03
6.96

7.03
6.96
14.65
3.92
3.09

3.09
8.71
0.00
8.62

0.00
8.62
5.01

0.00
5.01
15.26
6.19
8.63

6.19
8.63
8.40

$\begin{array}{ll}8.40 & 15 \\ 5.13 & 158\end{array}$

$\begin{array}{ll}5.40 & 158 \\ 5.13 & 130\end{array}$

$\begin{array}{ll}5.13 & 130 \\ 15.12 & 216 \\ 7.75 & 147\end{array}$

15.12
7.75
19.69

0.00
5.79
1.62

5.62
6.94

100 
Selective Sales--Alcoholic Beverages, Beer

\begin{tabular}{|c|c|c|c|c|c|c|c|c|}
\hline & $\begin{array}{l}1999 \text { State } \\
\text { Population }\end{array}$ & $\begin{array}{l}1999 \text { Base:Beer } \\
\text { Consumption } \\
\text { (millions of gallons) }\end{array}$ & $\begin{array}{l}1999 \text { Tax } \\
\text { Capacity } \\
\text { (\$ millions) }\end{array}$ & $\begin{array}{c}1999 \text { Capacity per capita } \\
\text { (\$/person) }\end{array}$ & 1999 Per Capita Capacity Index & $\begin{array}{l}1999 \text { Tax Revenue } \\
\text { (\$ millions) }\end{array}$ & $\begin{array}{c}1999 \text { Revenue per } \\
\text { capita } \\
\text { (\$/person) }\end{array}$ & 1999 Tax Effort Index \\
\hline Alabama & $4,369,862$ & 4.18 & 28.5 & 6.53 & 96.24 & 98.29 & 22.49 & 345 \\
\hline Alaska & 619,500 & 0.67 & 4.5 & 7.34 & 108.24 & 4.89 & 7.90 & 108 \\
\hline Arizona & $4,778,332$ & 5.72 & 39.0 & 8.16 & 120.28 & 20.53 & 4.30 & 53 \\
\hline Arkansas & $2,551,373$ & 2.32 & 15.8 & 6.20 & 91.46 & 15.77 & 6.18 & 100 \\
\hline California & $33,145,121$ & 28.54 & 194.6 & 5.87 & 86.57 & 124.42 & 3.75 & 64 \\
\hline Colorado & $4,056,133$ & 4.44 & 30.3 & 7.46 & 110.04 & 8.02 & 1.98 & 26 \\
\hline Connecticut & $3,282,031$ & 2.59 & 17.7 & 5.39 & 79.43 & 11.65 & 3.55 & 66 \\
\hline Delaware & 753,538 & 0.85 & 5.8 & 7.73 & 114.00 & 2.95 & 3.92 & 51 \\
\hline D.C. & 519,000 & 0.65 & 4.4 & 8.54 & 125.91 & 1.28 & 2.47 & 29 \\
\hline Florida & $15,111,244$ & 16.88 & 115.1 & 7.62 & 112.28 & 256.46 & 16.97 & 223 \\
\hline Georgia & $7,788,240$ & 7.69 & 52.5 & 6.74 & 99.32 & 174.94 & 22.46 & 333 \\
\hline Hawaii & $1,185,497$ & 1.29 & 8.8 & 7.40 & 109.06 & 25.01 & 21.10 & 285 \\
\hline Idaho & $1,251,700$ & 1.15 & 7.8 & 6.25 & 92.13 & 3.58 & 2.86 & 46 \\
\hline Illinois & $12,128,370$ & 12.55 & 85.6 & 7.06 & 104.04 & 38.94 & 3.21 & 45 \\
\hline Indiana & $5,942,901$ & 5.48 & 37.4 & 6.29 & 92.75 & 14.32 & 2.41 & 38 \\
\hline lowa & $2,869,413$ & 3.12 & 21.3 & 7.42 & 109.38 & 13.25 & 4.62 & 62 \\
\hline Kansas & $2,654,052$ & 2.37 & 16.2 & 6.09 & 89.79 & 39.83 & 15.01 & 246 \\
\hline Kentucky & $3,960,825$ & 3.45 & 23.6 & 5.95 & 87.68 & 6.17 & 1.56 & 26 \\
\hline Louisiana & $4,372,035$ & 5.34 & 36.4 & 8.32 & 122.74 & 39.82 & 9.11 & 109 \\
\hline Maine & $1,253,040$ & 1.19 & 8.1 & 6.46 & 95.32 & 9.08 & 7.25 & 112 \\
\hline Maryland & $5,171,634$ & 4.37 & 29.8 & 5.76 & 84.87 & 8.98 & 1.74 & 30 \\
\hline Massachusetts & $6,175,169$ & 5.72 & 39.0 & 6.32 & 93.12 & 13.48 & 2.18 & 35 \\
\hline Michigan & $9,863,775$ & 9.38 & 64.0 & 6.49 & 95.64 & 40.52 & 4.11 & 63 \\
\hline Minnesota & $4,775,508$ & 4.83 & 33.0 & 6.90 & 101.73 & 15.65 & 3.28 & 48 \\
\hline Mississippi & $2,768,619$ & 3.16 & 21.6 & 7.79 & 114.82 & 30.61 & 11.06 & 142 \\
\hline Missouri & $5,468,338$ & 5.94 & 40.5 & 7.40 & 109.12 & 7.93 & 1.45 & 20 \\
\hline Montana & 882,779 & 1.11 & 7.5 & 8.54 & 125.96 & 3.44 & 3.90 & 46 \\
\hline Nebraska & $1,666,028$ & 1.91 & 13.0 & 7.82 & 115.23 & 9.66 & 5.80 & 74 \\
\hline Nevada & $1,809,253$ & 2.73 & 18.6 & 10.30 & 151.81 & 5.27 & 2.92 & 28 \\
\hline New Hampshire & $1,201,134$ & 1.69 & 11.5 & 9.60 & 141.54 & 11.35 & 9.45 & 98 \\
\hline New Jersey & $8,143,412$ & 6.51 & 44.4 & 5.45 & 80.34 & 17.55 & 2.15 & 40 \\
\hline New Mexico & $1,739,844$ & 2.10 & 14.3 & 8.24 & 121.52 & 19.72 & 11.33 & 138 \\
\hline New York & $18,196,601$ & 14.18 & 96.7 & 5.31 & 78.32 & 60.66 & 3.33 & 63 \\
\hline North Carolina & $7,650,789$ & 7.30 & 49.8 & 6.50 & 95.89 & 82.38 & 10.77 & 166 \\
\hline North Dakota & 633,666 & 0.78 & 5.3 & 8.40 & 123.83 & 2.52 & 3.98 & 47 \\
\hline Ohio & $11,256,654$ & 11.96 & 81.5 & 7.24 & 106.80 & 52.31 & 4.65 & 64 \\
\hline Oklahoma & $3,358,044$ & 3.05 & 20.8 & 6.20 & 91.40 & 27.35 & 8.14 & 131 \\
\hline Oregon & $3,316,154$ & 3.30 & 22.5 & 6.78 & 99.89 & 6.17 & 1.86 & 27 \\
\hline Pennsylvania & $11,994,016$ & 12.08 & 82.4 & 6.87 & 101.27 & 25.48 & 2.12 & 31 \\
\hline Rhode Island & 990,819 & 0.96 & 6.6 & 6.63 & 97.81 & 2.55 & 2.57 & 39 \\
\hline South Carolina & $3,885,736$ & 4.52 & 30.8 & 7.94 & 117.04 & 74.80 & 19.25 & 243 \\
\hline South Dakota & 733,133 & 0.85 & 5.8 & 7.87 & 116.01 & 5.43 & 7.41 & 94 \\
\hline Tennessee & $5,483,535$ & 5.44 & 37.1 & 6.76 & 99.73 & 30.95 & 5.64 & 83 \\
\hline Texas & $20,044,141$ & 24.25 & 165.4 & 8.25 & 121.65 & 283.76 & 14.16 & 172 \\
\hline Utah & $2,129,836$ & 1.25 & 8.5 & 4.00 & 58.93 & 10.76 & 5.05 & 126 \\
\hline Vermont & 593,740 & 0.61 & 4.1 & 6.98 & 102.86 & 3.84 & 6.46 & 93 \\
\hline Virginia & $6,872,912$ & 6.61 & 45.0 & 6.55 & 96.63 & 39.98 & 5.82 & 89 \\
\hline Washington & $5,756,361$ & 5.15 & 35.1 & 6.10 & 89.89 & 30.64 & 5.32 & 87 \\
\hline West Virginia & $1,806,928$ & 1.77 & 12.1 & 6.69 & 98.65 & 7.21 & 3.99 & 60 \\
\hline Wisconsin & $5,250,446$ & 6.71 & 45.8 & 8.72 & 128.57 & 9.16 & 1.75 & 20 \\
\hline Wyoming & 479,602 & 0.55 & 3.8 & 7.83 & 115.50 & 0.23 & 0.48 & 6 \\
\hline Totals & 272690813.00 & 271.24 & $\begin{array}{l}1,849.6 \\
\end{array}$ & $\begin{array}{l}6.78 \\
\end{array}$ & 100.00 & $\begin{array}{l}1849.56 \\
\end{array}$ & 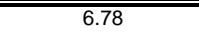 & 100 \\
\hline
\end{tabular}


1999 State Population 1999 Base:Wine Consumption 1999 Tax Capacity

Alabama

Alaska

Arizona
Arkansas

California

Colorado

Connecticut

Delaware

D.C.

Florida

Georgia

Hawail

Idaho

Illinois

Indian

lowa

Kentucky

Louisiana

Maine

Maryland
Massachusetts

Michigan

Minnesota

Mississippi

Missouri

Nebraska

Nevada

New Hampshire

New Jersey

New Mexico

North Carolina

North Dakota

Ohio

Oklahoma

Pregon

Pennsylvania

South Carolina

South Carolina

South Dakota

Texas

Utah

Vermont

Washington

West Virginia

Wisconsin

Totals

RTS rate

$$
\text { (millions of gallons) }
$$$$
0.56
$$

4,369,862

619,500
$4,778,332$

2,551,373

$33,145,121$
$4,056,133$

$3,282,031$

753,538

$5,111,244$

$7,788,240$

$1,185,497$

$1,251,700$

$12,128,370$

(2,869,413

2,654,052

$3,960,825$
$4,372,035$

$1,253,040$

$5,171,634$

$9,863,775$

$4,775,508$

$5,468,338$

882,779

$1,666,028$

$1,809,253$

$8,143,412$

$1,739,844$

$18,196,601$
$7,650,789$

$7,650,789$

$3,358,044$

$3,316,154$

$11,994,016$

$3,885,736$

$3,885,736$
733,133

$5,483,535$

20,044,141

$2,129,836$
593,740

$6,872,912$

$5,756,36$

$1,806,928$

$5,250,44$

479602.00

$\$ 6.54 /$ gallon per capita

1999 Per Capita

$$
51.40
$$

51.40
120.75

109.63

39.67

123.88

162.13

157.61

270.01

132.54

83.55
126.71

201.84

108.20

62.77

41.11

51.28
43.37
75.08
118.43

118.43

97.90

167.44

74.47

87.57
30.08

30.08
74.85

95.47

57.85

204.28

152.57

77.53

123.09
78.35

78.35
45.82
63.45

40.83

66.93

158.93

70.21

44.55

50.74

68.58

30.96

156.55
99.37

99.37
135.24

135.24
32.47

85.14

100.00
1999 Tax 1999 Revenue 1999 Tax Effort

Revenue per capita Index

$\begin{array}{ll} & \text { (\$/person) } \\ 7.19 & 197\end{array}$

$\begin{array}{ccc}7.19 & 1.65 & 197 \\ 1.17 & 1.89 & 97 \\ 7.97 & 1.67 & 94\end{array}$

$\begin{array}{ccc}7.97 & 1.67 & 94 \\ 2.49 & 0.98 & 152\end{array}$

$\begin{array}{lll}2.49 & 0.98 & 152 \\ 19.09 & 0.57 & 24\end{array}$

$\begin{array}{lll}2.496 & 0.57 & 24 \\ 2.94 & 0.73 & 36\end{array}$

6.68

$\begin{array}{ccc}6.15 & 2.85 & 111 \\ 0.86 & 1.66 & 38\end{array}$

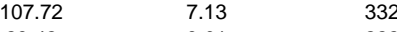

$\begin{array}{lll}23.42 & 3.01 & 222 \\ 4.35 & 3.67 & 179 \\ 2.47 & 1.97 & 60\end{array}$

$\begin{array}{lll}2.47 & 1.97 & 60 \\ 10.11 & 0.83 & 47 \\ 3.42 & 0.58 & 57\end{array}$

$\begin{array}{lll}10.11 & 0.83 & 47 \\ 3.42 & 0.58 & 57\end{array}$

$\begin{array}{lll}3.88 & 1.35 & 203\end{array}$

$\begin{array}{ccc}5.62 & 2.12 & 255 \\ 1.70 & 0.43 & 61\end{array}$

$\begin{array}{lll}1.45 & 0.33 & 27 \\ 1.86 & 1.48\end{array}$

$\begin{array}{lll}1.86 & 1.48 & 77 \\ 3.91 & 0.76 & 48\end{array}$

$\begin{array}{lll}11.16 & 1.81 & 67 \\ 7.29 & 0.74 & 6\end{array}$

$\begin{array}{lll}7.29 & 0.74 & 61 \\ 3.57 & 0.75 & 53\end{array}$

$\begin{array}{lll}1.18 & 0.42 & 87 \\ 2.80 & 0.51 & 42\end{array}$

$\begin{array}{llc}2.80 & 0.51 & 42 \\ 1.75 & 1.99 & 128 \\ 1.39 & 0.83 & 89\end{array}$

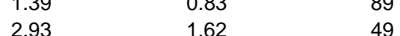

$\begin{array}{ccc}1.39 & 1.62 & 49 \\ 0.02 & 0.02 & 1\end{array}$

$\begin{array}{lll}16.86 & 2.07 & 84 \\ 4.61 & 2.65 & 211\end{array}$

$\begin{array}{lll}4.61 & 2.65 & 211 \\ 8.28 & 0.46 & 23 \\ .23 & 1.21\end{array}$

$\begin{array}{lll}9.23 & 1.21 & 95 \\ 0.33 & 0.52 & 70\end{array}$

$\begin{array}{lll}6.75 & 0.60 & 58\end{array}$

$\begin{array}{lll}5.23 & 1.56 & 235 \\ 5.65 & 1.71 & 73\end{array}$

$\begin{array}{lll}39.79 & 3.32 & 306\end{array}$

$\begin{array}{lll}1.90 & 1.92 & 74 \\ 6.43 & 1.06\end{array}$

$\begin{array}{lll}6.43 & 1.66 & 145 \\ 0.72 & 0.98 & 136\end{array}$

$\begin{array}{lll}12.95 & 2.36 & 287\end{array}$

$\begin{array}{lll}28.36 & 1.42 & 127 \\ 2.53 & 1.9 & 237\end{array}$

$\begin{array}{lll}1.05 & 1.19 & 237 \\ 20.14 & 2.93 & 70\end{array}$

$\begin{array}{lll}20.14 & 2.93 & 182 \\ 15.38 & 2.67 & 122\end{array}$

$\begin{array}{lll}1.14 & 0.63 & 120\end{array}$

$\begin{array}{ll}2.25 & 0.43 \\ 0.15 & 0.32\end{array}$

1.62

100 
Selective Sales--Tobacco

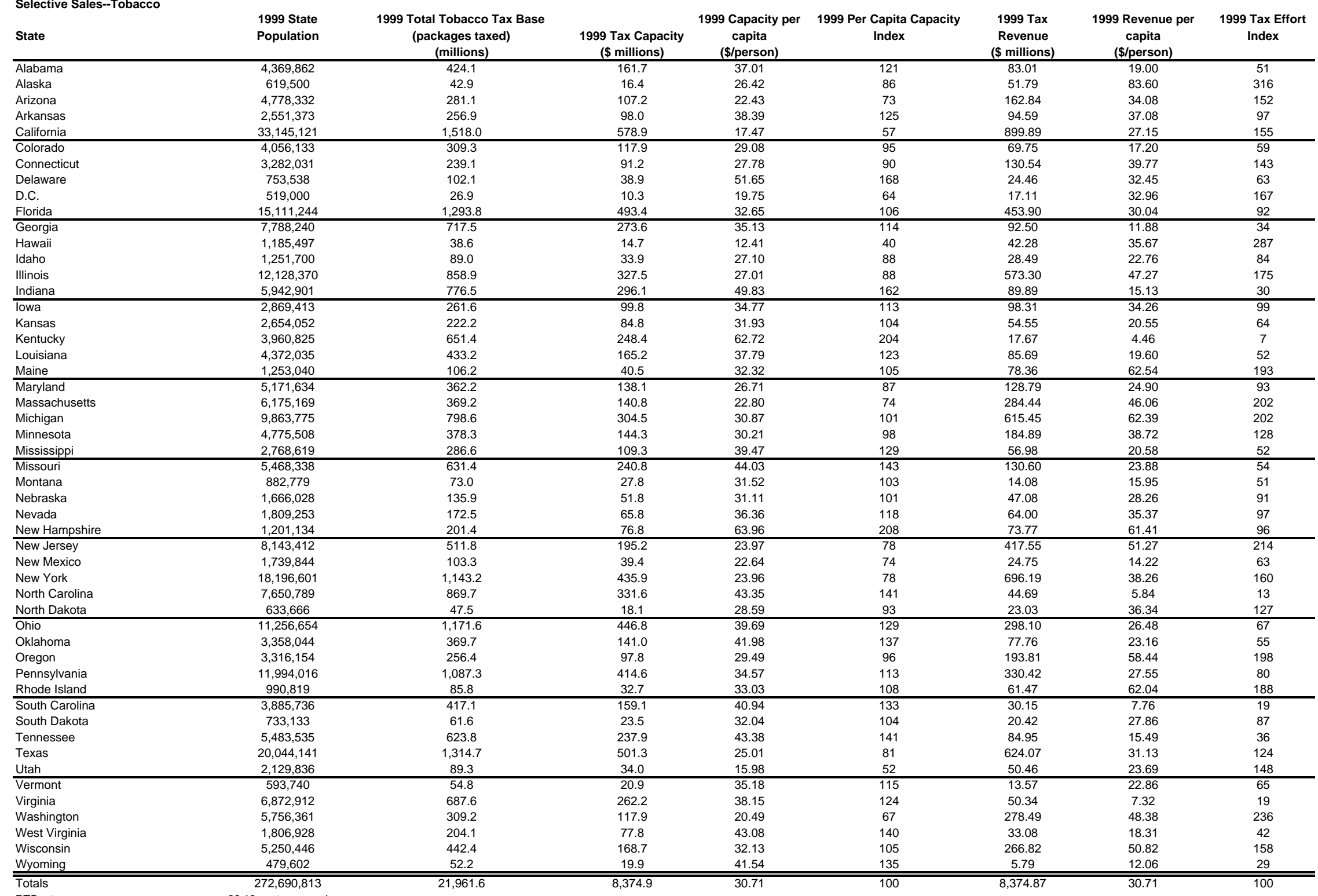

RTS rate

38.13 cents per pack 
Selective Sales--Insurance

\begin{tabular}{|c|c|c|c|c|c|c|c|c|c|c|}
\hline State & $\begin{array}{l}1999 \text { State } \\
\text { Population }\end{array}$ & $\begin{array}{l}1999 \text { Casuality/Property } \\
\text { Insurance } \\
(\$ \text { millions }) \\
\end{array}$ & $\begin{array}{l}1999 \text { Life } \\
\text { Insurance } \\
\text { (\$ millions) } \\
\end{array}$ & $\begin{array}{c}1999 \text { Total } \\
\text { Insurance Base } \\
\text { (\$ millions) }\end{array}$ & $\begin{array}{c}1999 \text { Tax } \\
\text { Capacity } \\
\text { (\$ millions) }\end{array}$ & $\begin{array}{c}1999 \text { Capacity per } \\
\text { capita } \\
\text { (\$/person) }\end{array}$ & $\begin{array}{l}1999 \text { Per Capita } \\
\text { Capacity Index }\end{array}$ & $\begin{array}{c}1999 \text { Tax } \\
\text { Revenue } \\
\text { (\$ millions) }\end{array}$ & $\begin{array}{c}1999 \text { Revenue } \\
\text { per capita } \\
\text { (\$/person) }\end{array}$ & $\begin{array}{l}1999 \text { Tax Effort } \\
\text { Index }\end{array}$ \\
\hline$\overline{\text { Alabama }}$ & $4,369,862$ & $4,004.0$ & $4,734.5$ & $8,738.5$ & 113.68 & 26.01 & 74 & 188.43 & 43.12 & 166 \\
\hline Alaska & 619,500 & 757.8 & 656.0 & $1,413.8$ & 18.39 & 29.69 & 84 & 28.39 & 45.83 & 154 \\
\hline Arizona & $4,778,332$ & $4,747.2$ & $7,011.5$ & $11,758.7$ & 152.97 & 32.01 & 91 & 151.88 & 31.79 & 99 \\
\hline Arkansas & $2,551,373$ & $2,493.4$ & $2,798.0$ & $5,291.4$ & 68.84 & 26.98 & 76 & 68.16 & 26.71 & 99 \\
\hline California & $33,145,121$ & $36,127.8$ & $43,108.0$ & $79,235.8$ & $1,030.78$ & 31.10 & 88 & $1,263.89$ & 38.13 & 123 \\
\hline Colorado & $4,056,133$ & $4,993.6$ & $7,376.0$ & $12,369.6$ & 160.92 & 39.67 & 112 & 120.61 & 29.73 & 75 \\
\hline Connecticut & $3,282,031$ & $4,619.5$ & $11,529.5$ & $16,149.0$ & 210.08 & 64.01 & 181 & 177.63 & 54.12 & 85 \\
\hline Delaware & 753,538 & $1,214.7$ & $4,003.0$ & $5,217.7$ & 67.88 & 90.08 & 255 & 51.13 & 67.86 & 75 \\
\hline D.C. & 519,000 & 895.6 & $2,467.5$ & $3,363.1$ & 43.75 & 84.30 & 239 & 25.67 & 49.46 & 59 \\
\hline Florida & $15,111,244$ & $19,212.5$ & $24,310.0$ & $43,522.5$ & 566.18 & 37.47 & 106 & 336.34 & 22.26 & 59 \\
\hline Georgia & $7,788,240$ & $7,920.4$ & $9,568.5$ & $17,488.9$ & 227.51 & 29.21 & 83 & 230.46 & 29.59 & 101 \\
\hline Hawaii & $1,185,497$ & $1,248.1$ & $1,680.0$ & $2,928.1$ & 38.09 & 32.13 & 91 & 54.04 & 45.58 & 142 \\
\hline Idaho & $1,251,700$ & $1,172.0$ & $1,803.0$ & $2,975.0$ & 38.70 & 30.92 & 88 & 52.32 & 41.80 & 135 \\
\hline Illinois & $12,128,370$ & $13,782.7$ & $26,388.0$ & $40,170.7$ & 522.58 & 43.09 & 122 & 218.19 & 17.99 & 42 \\
\hline Indiana & $5,942,901$ & $6,406.2$ & $8,424.5$ & $14,830.7$ & 192.93 & 32.46 & 92 & 157.14 & 26.44 & 81 \\
\hline lowa & $2,869,413$ & $2,849.9$ & $6,073.0$ & $8,922.9$ & 116.08 & 40.45 & 115 & 114.35 & 39.85 & 99 \\
\hline Kansas & $2,654,052$ & $2,832.9$ & $4,635.5$ & $7,468.4$ & 97.16 & 36.61 & 104 & 78.26 & 29.49 & 81 \\
\hline Kentucky & $\begin{array}{l}3,960,825 \\
\end{array}$ & $3,650.9$ & $3,715.5$ & $7,366.4$ & 95.83 & 24.19 & 69 & 212.76 & 53.72 & 222 \\
\hline Louisiana & $4,372,035$ & $4,987.4$ & $5,369.0$ & $10,356.4$ & 134.73 & 30.82 & 87 & 240.05 & 54.90 & 178 \\
\hline Maine & $1,253,040$ & $1,146.8$ & $1,484.0$ & $2,630.8$ & 34.22 & 27.31 & 77 & 41.87 & 33.41 & 122 \\
\hline Maryland & $5,171,634$ & $5,172.5$ & $8,943.5$ & $14,116.0$ & 183.63 & 35.51 & 101 & 178.08 & 34.43 & 97 \\
\hline Massachusetts & $6,175,169$ & $7,457.4$ & $17,806.0$ & $25,263.4$ & 328.65 & 53.22 & 151 & $\begin{array}{l}170.00 \\
336.37\end{array}$ & $\begin{array}{l}34.43 \\
54.47\end{array}$ & 102 \\
\hline Michigan & $9,863,775$ & $10,975.2$ & $15,251.5$ & $26,226.7$ & 341.18 & 34.59 & 98 & 199.46 & 20.22 & 58 \\
\hline Minnesota & $\begin{array}{l}4,775,508 \\
\end{array}$ & $5,681.3$ & $7,821.5$ & $13,502.8$ & 175.66 & 36.78 & 104 & 179.44 & 37.57 & 102 \\
\hline Mississippi & $2,768,619$ & $2,459.7$ & $3,085.0$ & $5,544.7$ & 72.13 & 26.05 & 74 & 111.67 & 40.33 & 155 \\
\hline Missouri & $5,468,338$ & $5,540.6$ & $8,732.0$ & $14,272.6$ & 185.67 & 33.95 & 96 & 216.58 & 39.61 & 117 \\
\hline Montana & 882,779 & 912.0 & 852.0 & $1,764.0$ & 22.95 & 25.99 & 74 & 38.85 & 44.01 & 169 \\
\hline Nebraska & $1,666,028$ & $1,972.8$ & $3,812.5$ & $5,785.3$ & 75.26 & 45.17 & 128 & 34.50 & 20.70 & 46 \\
\hline Nevada & $1,809,253$ & $1,973.8$ & $2,074.5$ & $4,048.3$ & 52.66 & 29.11 & 83 & 116.92 & 64.62 & 222 \\
\hline New Hampshire & $\begin{array}{l}1,201,134 \\
1,201\end{array}$ & $1,302.4$ & $2,394.5$ & $\begin{array}{l}4,040.0 \\
3,696.9\end{array}$ & 48.09 & 40.04 & 113 & 57.32 & 47.72 & 119 \\
\hline New Jersey & $8,143,412$ & $11,356.8$ & $19,117.5$ & $30,474.3$ & 396.44 & 48.68 & $\frac{1+5}{138}$ & 292.64 & 35.94 & 74 \\
\hline New Mexico & $1,739,844$ & $1,559.5$ & $1,675.5$ & $3,235.0$ & 42.08 & 24.19 & 69 & 81.39 & 46.78 & 193 \\
\hline New York & $18,196,601$ & $22,087.5$ & $36,529.0$ & $58,616.5$ & 762.54 & 41.91 & 119 & 716.58 & 39.38 & 94 \\
\hline North Carolina & $7,650,789$ & $7,310.6$ & $12,231.0$ & $19,541.6$ & 254.22 & 33.23 & 94 & 291.20 & 38.06 & 115 \\
\hline North Dakota & 633,666 & 760.2 & 853.5 & $1,613.7$ & 20.99 & 33.13 & 94 & 20.98 & 33.10 & 100 \\
\hline Ohio & $11,256,654$ & $11,136.7$ & $17,668.5$ & $28,805.2$ & 374.73 & 33.29 & 94 & 356.86 & 31.70 & 95 \\
\hline Oklahoma & $3,358,044$ & $3,233.3$ & $3,601.0$ & $6,834.3$ & 88.91 & 26.48 & 75 & 143.58 & 42.76 & 161 \\
\hline Oregon & $3,316,154$ & $3,599.5$ & $4,173.0$ & $7,772.5$ & 101.11 & 30.49 & 86 & 54.13 & 16.32 & 54 \\
\hline Pennsylvania & $11,994,016$ & $12,708.8$ & $22,559.5$ & $35,268.3$ & 458.80 & 38.25 & 108 & 432.18 & 36.03 & 94 \\
\hline Rhode Island & 990,819 & $1,168.1$ & $1,619.0$ & $\begin{array}{l}2,787.1 \\
\end{array}$ & 36.26 & 36.59 & 104 & 31.64 & 31.94 & 87 \\
\hline South Carolina & $3,885,736$ & $\frac{1,150.1}{4,159.4}$ & $4,367.5$ & $8,526.9$ & 110.93 & 28.55 & 81 & 79.72 & 20.52 & 72 \\
\hline South Dakota & 733,133 & 851.8 & $1,197.5$ & $2,049.3$ & 26.66 & 36.36 & 103 & 35.05 & 47.81 & 131 \\
\hline Tennessee & $5,483,535$ & $5,289.8$ & $7,416.5$ & $12,706.3$ & 165.30 & 30.14 & 85 & 257.22 & 46.91 & 156 \\
\hline Texas & $20,044,141$ & $20,285.6$ & $29,614.5$ & $49,900.1$ & 649.15 & 32.39 & 92 & 751.17 & 37.48 & 116 \\
\hline Utah & $2,129,836$ & $1,825.8$ & $2,512.0$ & $4,337.8$ & 56.43 & 26.49 & 75 & 77.66 & 36.46 & 138 \\
\hline Vermont & 593,740 & 647.7 & 878.0 & $1,525.7$ & 19.85 & 33.43 & 95 & 20.40 & 34.36 & 103 \\
\hline Virginia & $6,872,912$ & $6,065.0$ & $11,266.0$ & $17,331.0$ & 225.46 & 32.80 & 93 & 244.91 & 35.63 & 109 \\
\hline Washington & $5,756,361$ & $5,430.9$ & $7,368.0$ & $12,798.9$ & 166.50 & 28.92 & 82 & 238.99 & 41.52 & 144 \\
\hline West Virginia & $1,806,928$ & $1,546.8$ & $1,715.5$ & $3,262.3$ & 42.44 & 23.49 & 67 & 97.94 & 54.20 & 231 \\
\hline Wisconsin & $5,250,446$ & $5,503.9$ & $9,211.0$ & $14,714.9$ & 191.43 & 36.46 & 103 & 105.68 & 20.13 & 55 \\
\hline $\begin{array}{l}\text { Wyoming } \\
\text { Wyon }\end{array}$ & $\begin{array}{c}3,2000,440 \\
479,602\end{array}$ & $\begin{array}{l}360.4 \\
460.5\end{array}$ & 563.0 & $\begin{array}{c}1, / 14.9 \\
1,023.4 \\
\end{array}$ & 13.31 & 27.76 & 79 & $\begin{array}{l}10.04 \\
\end{array}$ & 20.94 & 75 \\
\hline$\overline{\text { Totals }}$ & $\overline{272,690,813}$ & 295,499.1 & 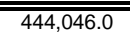 & $\overline{7739,545.1}$ & 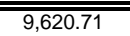 & 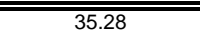 & $\overline{100}$ & 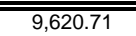 & 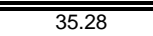 & 100 \\
\hline
\end{tabular}


Selective Sales-Public Utilities

\begin{tabular}{|c|c|c|c|c|c|c|c|c|c|c|c|}
\hline State & 1999 State Population & $\begin{array}{l}1999 \text { Gas } \\
\text { Revenue } \\
\text { (\$ millions) }\end{array}$ & $\begin{array}{l}1999 \text { Electric } \\
\text { Revenue } \\
\text { (\$ millions) }\end{array}$ & $\begin{array}{l}1999 \text { Telephone } \\
\text { Revenue } \\
\text { (\$ millions) }\end{array}$ & $\begin{array}{l}1999 \text { Base:Total } \\
\text { Revenue } \\
\text { ( } \$ \text { millions })\end{array}$ & $\begin{array}{l}1999 \text { Tax Capacity } \\
\text { (\$ millions) }\end{array}$ & $\begin{array}{l}1999 \text { Capacity per } \\
\text { capita } \\
\text { (\$/person) }\end{array}$ & $\begin{array}{l}1999 \text { Per Capita } \\
\text { Capacity Index }\end{array}$ & $\begin{array}{l}1999 \text { Tax } \\
\text { Revenue } \\
\text { (\$ millions) }\end{array}$ & $\begin{array}{l}1999 \text { Revenue } \\
\text { per capita } \\
\text { (\$/person) }\end{array}$ & $\begin{array}{l}1999 \text { Tax } \\
\text { Effort Index }\end{array}$ \\
\hline Alabama & $4,369,862$ & 612.1 & $4,430.1$ & $3,553.0$ & $8,595.3$ & 284.5 & 65.09 & 103 & 484.45 & 110.86 & 170 \\
\hline Alaska & 619,500 & 97.1 & 512.8 & 627.0 & $1,236.9$ & 40.9 & 66.08 & 105 & 3.69 & 5.96 & 9 \\
\hline Arizona & $4,778,332$ & 512.9 & $4,131.1$ & $4,158.5$ & $8,802.5$ & 291.3 & 60.97 & 97 & 149.22 & 31.23 & 51 \\
\hline Arkansas & $2,551,373$ & 427.1 & $2,266.5$ & $2,154.0$ & $4,847.7$ & 160.4 & 62.88 & 100 & 75.51 & 29.59 & 47 \\
\hline California & $33,145,121$ & $4,352.7$ & $20,115.3$ & $29,038.0$ & $53,506.0$ & $1,770.7$ & 53.42 & 85 & $1,995.14$ & 60.19 & 113 \\
\hline Colorado & $4,056,133$ & 806.1 & $2,385.6$ & $4,543.0$ & $7,734.6$ & 256.0 & 63.11 & 100 & 108.13 & 26.66 & 42 \\
\hline Connecticut & $3,282,031$ & 661.1 & $2,975.6$ & $3,289.0$ & $6,925.8$ & 229.2 & 69.84 & 111 & 167.70 & 51.10 & 73 \\
\hline Delaware & 753,538 & 141.2 & 731.3 & 736.5 & $1,609.0$ & 53.2 & 70.66 & 112 & 25.96 & 34.45 & 49 \\
\hline D.C. & 519,000 & 176.6 & 769.0 & $1,333.0$ & $2,278.7$ & 75.4 & 145.30 & 231 & 180.35 & 347.49 & 239 \\
\hline Florida & $15,111,244$ & 433.4 & $12,973.0$ & $16,132.5$ & $29,538.9$ & 977.6 & 64.69 & 103 & $1,998.99$ & 132.29 & 204 \\
\hline Georgia & $7,788,240$ & 525.2 & $7,056.0$ & $7,974.0$ & $15,555.2$ & 514.8 & 66.10 & 105 & 161.86 & 20.78 & 31 \\
\hline Hawaii & $1,185,497$ & 38.2 & $1,096.7$ & 989.0 & $2,123.9$ & 70.3 & 59.29 & 94 & 149.19 & 125.84 & 212 \\
\hline Idaho & $1,251,700$ & 189.8 & 862.4 & $1,051.0$ & $2,103.1$ & 69.6 & 55.61 & 88 & 12.61 & 10.08 & 18 \\
\hline Illinois & $12,128,370$ & $2,627.8$ & $9,508.7$ & $11,465.5$ & $23,602.0$ & 781.1 & 64.40 & 102 & $1,943.17$ & 160.22 & 249 \\
\hline Indiana & $5,942,901$ & $1,327.5$ & $5,015.2$ & $4,954.5$ & $11,297.2$ & 373.9 & 62.91 & 100 & 6.14 & 1.03 & 2 \\
\hline lowa & $2,869,413$ & 648.8 & $2,254.8$ & $2,354.5$ & $5,258.0$ & 174.0 & 60.64 & 96 & 14.92 & 5.20 & 9 \\
\hline Kansas & $2,654,052$ & $1,167.8$ & $2,123.4$ & $2,446.0$ & $5,737.2$ & 189.9 & 71.54 & 114 & 102.65 & 38.68 & 54 \\
\hline Kentucky & $3,960,825$ & 478.7 & $3,226.8$ & $3,243.0$ & $6,948.5$ & 230.0 & 58.06 & 92 & 147.58 & 37.26 & 64 \\
\hline Louisiana & $4,372,035$ & 762.9 & $4,519.9$ & $3,672.5$ & $8,955.3$ & 296.4 & 67.79 & 108 & 141.28 & 32.31 & 48 \\
\hline Maine & $1,253,040$ & 29.9 & $1,156.7$ & $1,150.0$ & $2,336.6$ & 77.3 & 61.71 & 98 & 0.62 & 0.49 & 1 \\
\hline Maryland & $5,171,634$ & 669.0 & $4,101.4$ & $5,043.5$ & $9,814.0$ & 324.8 & 62.80 & 100 & 293.29 & 56.71 & 90 \\
\hline Massachusetts & $6,175,169$ & $1,344.1$ & $4,520.8$ & $6,449.5$ & $12,314.4$ & 407.5 & 66.00 & 105 & 0.00 & 0.00 & 0 \\
\hline Michigan & $9,863,775$ & $2,469.1$ & $7,258.4$ & $9,026.5$ & $18,754.0$ & 620.7 & 62.92 & 100 & 50.92 & 5.16 & 8 \\
\hline Minnesota & $4,775,508$ & $1,097.4$ & $3,291.4$ & $4,366.0$ & $8,754.8$ & 289.7 & 60.67 & 96 & 52.30 & 10.95 & 18 \\
\hline Mississippi & $2,768,619$ & 319.4 & $2,514.2$ & $2,150.0$ & $4,983.6$ & 164.9 & 59.57 & 95 & 31.19 & 11.27 & 19 \\
\hline Missouri & $5,468,338$ & $1,054.6$ & $4,189.5$ & $5,027.5$ & $10,271.6$ & 339.9 & 62.16 & 99 & 331.53 & 60.63 & 98 \\
\hline Montana & 882,779 & 154.9 & 634.3 & 838.5 & $1,627.6$ & 53.9 & 61.02 & 97 & 16.55 & 18.74 & 31 \\
\hline Nebraska & $1,666,028$ & 350.4 & $1,219.5$ & $1,662.0$ & $3,231.9$ & 107.0 & 64.20 & 102 & 44.45 & 26.68 & 42 \\
\hline Nevada & $1,809,253$ & 291.3 & $1,498.8$ & $1,738.0$ & $3,528.1$ & 116.8 & 64.54 & 102 & 73.74 & 40.75 & 63 \\
\hline New Hampshire & $1,201,134$ & 116.3 & $1,123.0$ & $1,279.5$ & $2,518.8$ & 83.4 & 69.40 & 110 & 56.58 & 47.10 & 68 \\
\hline New Jersey & $8,143,412$ & $2,435.1$ & $6,993.1$ & $9,462.0$ & $18,890.2$ & 625.2 & 76.77 & 122 & $1,096.47$ & 134.65 & 175 \\
\hline New Mexico & $1,739,844$ & 265.8 & $1,208.6$ & $1,475.5$ & $2,949.9$ & 97.6 & 56.11 & 89 & 40.90 & 23.51 & 42 \\
\hline New York & $18,196,601$ & $4,648.3$ & $13,773.2$ & $18,817.5$ & $37,239.0$ & $1,232.4$ & 67.73 & 108 & $2,093.27$ & 115.04 & 170 \\
\hline North Carolina & $7,650,789$ & 862.6 & $7,372.1$ & $7,651.5$ & $15,886.1$ & 525.7 & 68.72 & 109 & 344.32 & 45.00 & 65 \\
\hline North Dakota & 633,666 & 100.0 & 484.7 & 629.5 & $1,214.2$ & 40.2 & 63.41 & 101 & 32.15 & 50.74 & 80 \\
\hline Ohio & $11,256,654$ & $2,388.9$ & $10,357.1$ & $9,674.0$ & $22,420.0$ & 742.0 & 65.91 & 105 & 696.46 & 61.87 & 94 \\
\hline Oklahoma & $3,358,044$ & 593.0 & $2,556.4$ & $2,639.5$ & $5,788.9$ & 191.6 & 57.05 & 91 & 91.29 & 27.19 & 48 \\
\hline Oregon & $3,316,154$ & 533.4 & $2,248.1$ & $3,014.0$ & $5,795.5$ & 191.8 & 57.84 & 92 & 118.21 & 35.65 & 62 \\
\hline Pennsylvania & $11,994,016$ & $2,300.6$ & $8,643.0$ & $10,539.5$ & $21,483.1$ & 711.0 & 59.28 & 94 & 762.44 & 63.57 & 107 \\
\hline Rhode Island & 990,819 & 227.1 & 628.9 & 902.5 & $1,758.5$ & 58.2 & 58.74 & 93 & 66.59 & 67.20 & 114 \\
\hline South Carolina & $3,885,736$ & 489.0 & $4,047.0$ & $3,591.5$ & $8,127.4$ & 269.0 & 69.22 & 110 & 92.94 & 23.92 & 35 \\
\hline South Dakota & 733,133 & 100.4 & 496.2 & 675.5 & $1,272.1$ & 42.1 & 57.42 & 91 & 2.87 & 3.91 & 7 \\
\hline Tennessee & $5,483,535$ & 877.6 & $5,198.6$ & $4,740.5$ & $10,816.7$ & 358.0 & 65.28 & 104 & 53.15 & 9.69 & 15 \\
\hline Texas & $20,044,141$ & $2,488.4$ & $18,364.8$ & $18,304.0$ & $39,157.2$ & $1,295.9$ & 64.65 & 103 & $1,174.81$ & 58.61 & 91 \\
\hline Utah & $2,129,836$ & 793.0 & $1,066.3$ & $1,673.5$ & $3,532.7$ & 116.9 & 54.89 & 87 & 68.78 & 32.29 & 59 \\
\hline Vermont & 593,740 & 39.9 & 547.7 & 643.0 & $1,230.6$ & 40.7 & 68.59 & 109 & 9.17 & 15.45 & 23 \\
\hline Virginia & $6,872,912$ & 882.6 & $5,389.1$ & $6,798.0$ & $13,069.7$ & 432.5 & 62.93 & 100 & 526.60 & 76.62 & 122 \\
\hline Washington & $5,756,361$ & 689.2 & $3,767.6$ & $5,391.5$ & $9,848.3$ & 325.9 & 56.62 & 90 & 553.66 & 96.18 & 170 \\
\hline West Virginia & $1,806,928$ & 331.3 & $1,363.8$ & $1,410.0$ & $3,105.2$ & 102.8 & 56.87 & 90 & 220.49 & 122.02 & 215 \\
\hline Wisconsin & $5,250,446$ & $1,298.4$ & $3,445.4$ & $4,476.5$ & $9,220.2$ & 305.1 & 58.12 & 92 & 294.83 & 56.15 & 97 \\
\hline Wyoming & 479,602 & 107.2 & 504.2 & 487.5 & $1,098.8$ & 36.4 & 75.82 & 120 & 11.26 & 23.47 & 31 \\
\hline Totals & $272,690,813$ & $446,335.2$ & $\overline{217,052.7}$ & $=255,441.0$ & $\overline{~ 518,828.9}$ & 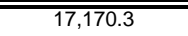 & 262.97 & 100 & $\bar{~} \overline{17,170.34}$ & 262.97 & 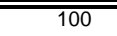 \\
\hline
\end{tabular}




\begin{tabular}{|c|c|c|c|c|c|c|c|c|c|c|c|}
\hline State & 1999 State Population & $\begin{array}{l}1999 \text { Horse } \\
\text { Handle } \\
\text { (\$ millions) }\end{array}$ & $\begin{array}{l}1999 \text { Dog Handle } \\
\text { (\$ millions) }\end{array}$ & $\begin{array}{l}1999 \text { Jai-Alai } \\
\text { Handle } \\
\text { (\$ millions) }\end{array}$ & $\begin{array}{l}1999 \text { Base:Total Pari- } \\
\text { Mutuel Handle } \\
\text { (\$ millions) }\end{array}$ & $\begin{array}{l}1999 \text { Tax } \\
\text { Capacity } \\
\text { (\$ millions) }\end{array}$ & $\begin{array}{c}1999 \\
\text { Capacity per } \\
\text { capita } \\
\text { (S/person) }\end{array}$ & $\begin{array}{l}1999 \text { Per Capita } \\
\text { Capacity Index }\end{array}$ & $\begin{array}{c}1999 \text { Tax } \\
\text { Revenue } \\
\text { (\$ millions) }\end{array}$ & $\begin{array}{c}1999 \text { Revenue per } \\
\text { capita } \\
\text { (\$/person) }\end{array}$ & $\begin{array}{l}1999 \text { Tax Effort } \\
\text { Index }\end{array}$ \\
\hline Alabama & $4,369,862$ & 45.9 & 224.1 & 0.0 & 270.0 & 5.68 & 1.30 & 93 & 3.82 & 0.87 & 67 \\
\hline Alaska & 619,500 & 0.0 & 0.0 & 0.0 & 0.0 & 0.00 & 0.00 & 0 & 0.00 & 0.00 & 0 \\
\hline Arizona & $4,778,332$ & 139.4 & 130.4 & 0.0 & 269.8 & 5.68 & 1.19 & 85 & 2.95 & 0.62 & 52 \\
\hline Arkansas & $2,551,373$ & 129.1 & 109.4 & 0.0 & 238.5 & 5.02 & 1.97 & 140 & 6.23 & 2.44 & 124 \\
\hline California & $33,145,121$ & $2,410.6$ & 0.0 & 0.0 & $2,410.6$ & 50.73 & 1.53 & 109 & 64.08 & 1.93 & 126 \\
\hline Colorado & $4,056,133$ & 75.6 & 124.7 & 0.0 & 200.3 & 4.22 & 1.04 & 74 & 6.11 & 1.51 & 145 \\
\hline Connecticut & $3,282,031$ & 205.7 & 58.8 & 65.9 & 330.3 & 6.95 & 2.12 & 151 & 11.03 & 3.36 & 159 \\
\hline Delaware & 753,538 & 150.2 & 0.0 & 0.0 & 150.2 & 3.16 & 4.19 & 299 & 0.21 & 0.27 & 7 \\
\hline D.C. & 519,000 & 0.0 & 0.0 & 0.0 & 0.0 & 0.00 & 0.00 & 0 & 0.00 & 0.00 & 0 \\
\hline Florida & $15,111,244$ & 870.5 & 693.1 & 135.1 & $1,698.7$ & 35.75 & 2.37 & 169 & 50.78 & 3.36 & 142 \\
\hline Georgia & $7,788,240$ & 0.0 & 0.0 & 0.0 & 0.0 & 0.00 & 0.00 & 0 & 0.00 & 0.00 & 0 \\
\hline Hawaii & $1,185,497$ & 0.0 & 0.0 & 0.0 & 0.0 & 0.00 & 0.00 & 0 & 0.00 & 0.00 & 0 \\
\hline Idaho & $1,251,700$ & 30.9 & 4.3 & 0.0 & 35.2 & 0.74 & 0.59 & 42 & 0.00 & 0.00 & 0 \\
\hline Illinois & $12,128,370$ & $1,103.8$ & 0.0 & 0.0 & $1,103.8$ & 23.23 & 1.92 & 137 & 37.05 & 3.05 & 160 \\
\hline Indiana & $5,942,901$ & 155.3 & 0.0 & 0.0 & 155.3 & 3.27 & 0.55 & 39 & 3.67 & 0.62 & 112 \\
\hline Iowa & $2,869,413$ & 39.1 & 27.8 & 0.0 & 66.9 & 1.41 & 0.49 & 35 & 3.35 & 1.17 & 238 \\
\hline Kansas & $2,654,052$ & 46.4 & 69.4 & 0.0 & 115.9 & 2.44 & 0.92 & 66 & 4.12 & 1.55 & 169 \\
\hline Kentucky & $3,960,825$ & 630.8 & 0.0 & 0.0 & 630.8 & 13.27 & 3.35 & 239 & 26.76 & 6.75 & 202 \\
\hline Louisiana & $4,372,035$ & 397.6 & 0.0 & 0.0 & 397.6 & 8.37 & 1.91 & 137 & 5.41 & 1.24 & 65 \\
\hline Maine & $1,253,040$ & 69.2 & 0.0 & 0.0 & 69.2 & 1.46 & 1.16 & 83 & 4.67 & 3.72 & 320 \\
\hline Maryland & $5,171,634$ & 567.2 & 0.0 & 0.0 & 567.2 & 11.94 & 2.31 & 165 & 0.72 & 0.14 & 6 \\
\hline Massachusetts & $6,175,169$ & 219.0 & 197.6 & 0.0 & 416.6 & 8.77 & 1.42 & 101 & 8.27 & 1.34 & 94 \\
\hline Michigan & $9,863,775$ & 416.6 & 0.0 & 0.0 & 416.6 & 8.77 & 0.89 & 63 & 13.29 & 1.35 & 152 \\
\hline Minnesota & $4,775,508$ & 72.2 & 0.0 & 0.0 & 72.2 & 1.52 & 0.32 & 23 & 0.88 & 0.18 & 58 \\
\hline Mississippi & $2,768,619$ & 0.0 & 0.0 & 0.0 & 0.0 & 0.00 & 0.00 & 0 & 0.00 & 0.00 & 0 \\
\hline Missouri & $5,468,338$ & 0.0 & 0.0 & 0.0 & 0.0 & 0.00 & 0.00 & 0 & 0.00 & 0.00 & 0 \\
\hline Montana & 882,779 & 10.9 & 0.0 & 0.0 & 10.9 & 0.23 & 0.26 & 19 & 0.13 & 0.15 & 57 \\
\hline Nebraska & $1,666,028$ & 112.0 & 0.0 & 0.0 & 112.0 & 2.36 & 1.41 & 101 & 0.70 & 0.42 & 30 \\
\hline Nevada & $1,809,253$ & 502.3 & 0.0 & 0.0 & 502.3 & 10.57 & 5.84 & 417 & 0.00 & 0.00 & 0 \\
\hline New Hampshire & $1,201,134$ & 173.9 & 69.9 & 0.0 & 243.7 & 5.13 & 4.27 & 305 & 3.64 & 3.03 & 71 \\
\hline New Jersey & $8,143,412$ & $1,228.1$ & 0.0 & 0.0 & $1,228.1$ & 25.84 & 3.17 & 226 & 0.00 & 0.00 & 0 \\
\hline New Mexico & $1,739,844$ & 98.8 & 0.0 & 0.0 & 98.8 & 2.08 & 1.20 & 85 & 0.70 & 0.40 & 34 \\
\hline New York & $18,196,601$ & $2,746.0$ & 0.0 & 0.0 & $2,746.0$ & 57.79 & 3.18 & 227 & 36.92 & 2.03 & 64 \\
\hline North Carolina & $7,650,789$ & 0.0 & 0.0 & 0.0 & 0.0 & 0.00 & 0.00 & 0 & 0.00 & 0.00 & 0 \\
\hline North Dakota & 633,666 & 88.6 & 0.0 & 0.0 & 88.6 & 1.86 & 2.94 & 210 & 0.00 & 0.00 & 0 \\
\hline Ohio & $11,256,654$ & 627.7 & 0.0 & 0.0 & 627.7 & 13.21 & 1.17 & 84 & 16.53 & 1.47 & 125 \\
\hline Oklahoma & $3,358,044$ & 217.5 & 0.0 & 0.0 & 217.5 & 4.58 & 1.36 & 97 & 4.37 & 1.30 & 95 \\
\hline Oregon & $3,316,154$ & 60.1 & 38.9 & 0.0 & 99.0 & 2.08 & 0.63 & 45 & 1.20 & 0.36 & 58 \\
\hline Pennsylvania & $11,994,016$ & $1,139.5$ & 0.0 & 0.0 & $1,139.5$ & 23.98 & 2.00 & 143 & 30.18 & 2.52 & 126 \\
\hline Rhode Island & $\begin{array}{c}1,20,819 \\
990,0\end{array}$ & 68.4 & 32.9 & 2.1 & 103.4 & 2.18 & 2.20 & 157 & 5.81 & 5.86 & 267 \\
\hline South Carolina & $3,885,736$ & 0.0 & 0.0 & 0.0 & 0.0 & 0.00 & 0.00 & 0 & 0.00 & 0.00 & 0 \\
\hline South Dakota & 733,133 & 25.6 & 12.3 & 0.0 & 37.9 & 0.80 & 1.09 & 78 & 1.76 & 2.40 & 221 \\
\hline Tennessee & $5,483,535$ & 0.0 & 0.0 & 0.0 & 0.0 & 0.00 & 0.00 & 0 & 0.00 & 0.00 & 0 \\
\hline Texas & $20,044,141$ & 478.9 & 145.9 & 0.0 & 624.9 & 13.15 & 0.66 & 47 & 13.34 & 0.67 & 101 \\
\hline Utah & $2,129,836$ & 0.0 & 0.0 & 0.0 & 0.0 & 0.00 & 0.00 & 0 & 0.00 & 0.00 & 0 \\
\hline Vermont & 593,740 & 0.0 & 0.0 & 0.0 & 0.0 & 0.00 & 0.00 & 0 & 0.00 & 0.00 & 0 \\
\hline Virginia & $6,872,912$ & 121.3 & 0.0 & 0.0 & 121.3 & 2.55 & 0.37 & 27 & 0.00 & 0.00 & 0 \\
\hline Washington & $5,756,361$ & 158.5 & 0.0 & 0.0 & 158.5 & 3.33 & 0.58 & 41 & 1.91 & 0.33 & 57 \\
\hline West Virginia & $1,806,928$ & 121.2 & 97.8 & 0.0 & 219.0 & 4.61 & 2.55 & 182 & 7.95 & 4.40 & 172 \\
\hline Wisconsin & $5,250,446$ & 61.3 & 92.8 & 0.0 & 154.1 & 3.24 & 0.62 & 44 & 3.39 & 0.65 & 104 \\
\hline Wyoming & $\begin{array}{l}479,602 \\
\end{array}$ & 12.0 & 0.0 & 0.0 & 12.0 & 0.25 & 0.52 & 37 & 0.29 & 0.61 & 117 \\
\hline Totals & $272,690,813$ & 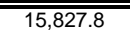 & $2,130.1$ & 203.0 & \begin{tabular}{ll|}
$18,160.9$ \\
\end{tabular} & "382.17 & $\begin{array}{l}1.40 \\
\end{array}$ & 100 & ב382.17 & $\overline{c 1.40}$ & $\overline{100}$ \\
\hline
\end{tabular}




\begin{tabular}{|c|c|c|c|c|c|c|c|c|}
\hline State & 1999 State Population & $\begin{array}{c}1999 \text { Total Amusements Base-- } \\
\text { Adjusted } \\
\text { (\$ millions) }\end{array}$ & $\begin{array}{c}1999 \text { Tax } \\
\text { Capacity } \\
\text { (\$ millions) }\end{array}$ & $\begin{array}{c}1999 \text { Capacity per } \\
\text { capita } \\
\text { (\$/person) }\end{array}$ & $\begin{array}{l}1999 \text { Per Capita Capacity } \\
\text { Index }\end{array}$ & $\begin{array}{c}1999 \text { Tax } \\
\text { Revenue } \\
\text { (\$ millions) }\end{array}$ & $\begin{array}{c}1999 \text { Revenue per } \\
\text { capita } \\
\text { (\$/person) }\end{array}$ & $\begin{array}{l}1999 \text { Tax Effort } \\
\text { Index }\end{array}$ \\
\hline$\overline{\text { Alabama }}$ & $4,369,862$ & 515.2 & 19.1 & 4.36 & 36 & 0.09 & 0.02 & 0 \\
\hline Alaska & 619,500 & 199.3 & 7.4 & 11.90 & 99 & 2.19 & 3.54 & 30 \\
\hline Arizona & $4,778,332$ & $2,685.6$ & 99.4 & 20.79 & 172 & 0.72 & 0.15 & 1 \\
\hline Arkansas & $2,551,373$ & 288.1 & 10.7 & 4.18 & 35 & 0.22 & 0.09 & 2 \\
\hline California & $33,145,121$ & $12,756.1$ & 472.0 & 14.24 & 118 & 0.00 & 0.00 & 0 \\
\hline Colorado & $4,056,133$ & $2,509.3$ & 92.8 & 22.89 & 190 & 72.99 & 17.99 & 79 \\
\hline Connecticut & $3,282,031$ & $2,743.4$ & 101.5 & 30.93 & 256 & 291.03 & 88.67 & 287 \\
\hline Delaware & 753,538 & 298.8 & 11.1 & 14.67 & 122 & 0.00 & 0.00 & 0 \\
\hline D.C. & 519,000 & 136.1 & 5.0 & 9.71 & 80 & 0.00 & 0.00 & 0 \\
\hline Florida & $15,111,244$ & $8,686.1$ & 321.4 & 21.27 & 176 & 0.00 & 0.00 & 0 \\
\hline Georgia & $7,788,240$ & $1,641.8$ & 60.7 & 7.80 & 65 & 0.00 & 0.00 & 0 \\
\hline Hawaii & $1,185,497$ & 410.6 & 15.2 & 12.82 & 106 & 0.00 & 0.00 & 0 \\
\hline Idaho & $1,251,700$ & 183.8 & 6.8 & 5.43 & 45 & 0.00 & 0.00 & 0 \\
\hline Illinois & $12,128,370$ & $3,748.6$ & 138.7 & 11.44 & 95 & 375.33 & 30.95 & 271 \\
\hline Indiana & $5,942,901$ & $2,507.5$ & 92.8 & 15.61 & 129 & 405.93 & 68.30 & 438 \\
\hline lowa & $2,869,413$ & $\begin{array}{l}2,011.4 \\
1,01\end{array}$ & 37.4 & 13.04 & 108 & 153.76 & 53.59 & 411 \\
\hline Kansas & $2,654,052$ & $\begin{array}{c}470.9 \\
470.9\end{array}$ & 17.4 & 6.56 & 54 & $\begin{array}{c}153.10 \\
0.98\end{array}$ & 0.37 & $\begin{array}{c}411 \\
6\end{array}$ \\
\hline Kentucky & $3,960,825$ & 685.2 & 25.4 & 6.40 & 53 & 0.24 & 0.06 & 1 \\
\hline Louisiana & $4,372,035$ & $2,252.7$ & 83.3 & 19.06 & 158 & 462.03 & 105.68 & 554 \\
\hline Maine & $1,253,040$ & 322.0 & 11.9 & 9.51 & 79 & 0.00 & 0.00 & 0 \\
\hline Maryland & $5,171,634$ & $1,384.2$ & 51.2 & 9.90 & 82 & 10.10 & 1.95 & 20 \\
\hline Massachusetts & $6,175,169$ & $1,755.9$ & 65.0 & 10.52 & 87 & 6.52 & 1.06 & 10 \\
\hline Michigan & $9,863,775$ & $2,649.3$ & 98.0 & 9.94 & 82 & 5.99 & 0.61 & 6 \\
\hline Minnesota & $\begin{array}{l}4,775,508 \\
\end{array}$ & $1,530.2$ & 56.6 & 11.86 & 98 & 62.76 & 13.14 & 111 \\
\hline Mississippi & $2,768,619$ & $1,453.2$ & 53.8 & 19.42 & 161 & 187.34 & 67.67 & 348 \\
\hline Missouri & $5,468,338$ & $1,818.4$ & 67.3 & 12.30 & 102 & 164.66 & 30.11 & 245 \\
\hline Montana & $\begin{array}{l}882,779 \\
\end{array}$ & $\begin{array}{c}59.9 \\
59.4\end{array}$ & 2.2 & 2.51 & 21 & 37.85 & 42.87 & 1,709 \\
\hline Nebraska & $1,666,028$ & 296.9 & 11.0 & 6.59 & 55 & 6.32 & 3.79 & 58 \\
\hline Nevada & $1,809,253$ & $1,617.7$ & 59.9 & 33.08 & 274 & 633.80 & 350.31 & 1,059 \\
\hline New Hampshire & $1,201,134$ & 413.1 & 15.3 & 12.73 & 105 & 1.83 & 1.52 & 12 \\
\hline New Jersey & $\frac{x, 143,412}{8,14}$ & $1,987.4$ & 73.5 & 9.03 & 75 & 326.46 & 40.09 & 444 \\
\hline New Mexico & $1,739,844$ & 704.8 & 26.1 & 14.99 & 124 & 2.89 & 1.66 & 11 \\
\hline New York & $18,196,601$ & $6,534.7$ & 241.8 & 13.29 & 110 & 0.69 & 0.04 & 0 \\
\hline North Carolina & $7,650,789$ & $2,018.5$ & 74.7 & 9.76 & 81 & 0.00 & 0.00 & 0 \\
\hline North Dakota & 633,666 & 186.6 & 6.9 & 10.89 & 90 & 11.15 & 17.59 & 161 \\
\hline Ohio & $11,256,654$ & $2,717.7$ & 100.6 & 8.93 & 74 & 0.00 & 0.00 & 0 \\
\hline Oklahoma & $3,358,044$ & 694.4 & 25.7 & 7.65 & 63 & 8.43 & 2.51 & 33 \\
\hline Oregon & $3,316,154$ & $1,013.2$ & 37.5 & 11.30 & 94 & 0.11 & 0.03 & 0 \\
\hline Pennsylvania & $11,994,016$ & $2,729.5$ & 101.0 & 8.42 & 70 & 0.69 & 0.06 & 1 \\
\hline Rhode Island & $\begin{array}{c}990,819 \\
\end{array}$ & 261.1 & 9.7 & 9.75 & 81 & 0.00 & 0.00 & 0 \\
\hline South Carolina & $3,885,736$ & $1,252.0$ & 46.3 & 11.92 & 99 & 34.76 & 8.95 & 75 \\
\hline South Dakota & 733,133 & 328.7 & 12.2 & 16.59 & 137 & 0.02 & 0.03 & 0 \\
\hline Tennessee & $5,483,535$ & $1,312.1$ & 48.5 & 8.85 & 73 & 0.00 & 0.00 & 0 \\
\hline Texas & $20,044,141$ & $4,409.7$ & 163.2 & 8.14 & 67 & 22.96 & 1.15 & 14 \\
\hline Utah & $2,129,836$ & 473.5 & 17.5 & 8.22 & 68 & 0.00 & 0.00 & 0 \\
\hline Vermont & 593,740 & 271.9 & 10.1 & 16.95 & 140 & 0.00 & 0.00 & 0 \\
\hline Virginia & $6,872,912$ & $1,578.0$ & 58.4 & 8.49 & 70 & 0.11 & 0.02 & 0 \\
\hline Washington & $5,756,361$ & $1,923.1$ & 71.2 & 12.36 & 102 & 0.00 & 0.00 & 0 \\
\hline West Virginia & $1,806,928$ & 344.8 & 12.8 & 7.06 & 58 & 0.00 & 0.00 & 0 \\
\hline Wisconsin & $5,250,446$ & $1,635.7$ & 60.5 & 11.53 & 95 & 0.57 & 0.11 & 1 \\
\hline Wyoming & 479,602 & 44.4 & 1.6 & 3.42 & 28 & 0.00 & 0.00 & 0 \\
\hline $\begin{array}{ll}\text { Totals } \\
\end{array}$ & $\overline{272,690,813}$ & $\overline{88,962.4}$ & 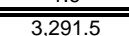 & 12.07 & 100 & $\overline{3,291.50}$ & 12.07 & 100 \\
\hline
\end{tabular}

RTS rate $\quad 3.70$ cents per dollar 
Selective Sales--Adjustments to Amusements, Sales--(receipts \$1000s)

\begin{tabular}{|c|c|c|c|c|c|c|c|}
\hline$\frac{\text { NAICS Code: }}{\text { Alabama }}$ & $\begin{array}{c}\mathbf{7 1} \\
435,740\end{array}$ & $\begin{array}{c}7113 \\
28,648\end{array}$ & $\begin{array}{l}7114 \\
5,051\end{array}$ & $\begin{array}{l}7115 \\
4,420\end{array}$ & $\begin{array}{c}7139905 \\
15,887\end{array}$ & $\begin{array}{r}51213 \\
57,409\end{array}$ & 439,143 \\
\hline Alaska & 168,275 & 4,357 & 0 & 1,624 & 1,327 & 25,065 & 186,032 \\
\hline Arizona & $2,033,290$ & 125,879 & 9,175 & 35,230 & 15,898 & 169,564 & $2,016,672$ \\
\hline Arkansas & 228,733 & 5,004 & 1,406 & 3,135 & 10,544 & 44,785 & 253,429 \\
\hline California & $15,913,820$ & 813,429 & 974,826 & $3,853,117$ & 110,047 & $1,297,474$ & $11,459,875$ \\
\hline Colorado & $1,909,633$ & 124,570 & 12,893 & 36,120 & 22,930 & 140,991 & $1,854,111$ \\
\hline Connecticut & $2,526,777$ & 127,459 & 20,430 & 51,205 & 9,634 & 98,494 & $2,416,543$ \\
\hline Delaware* & 240,140 & 0 & 0 & 0 & 2,965 & 17,131 & 254,306 \\
\hline D.C. & 161,944 & 59,298 & 22,881 & 20,273 & 0 & 23,186 & 82,678 \\
\hline Florida & $7,871,475$ & 414,764 & 132,176 & 130,623 & 46,736 & 438,363 & $7,585,539$ \\
\hline Georgia & $1,533,747$ & 236,642 & 16,097 & 41,073 & 45,334 & 170,100 & $1,364,701$ \\
\hline Hawaii & 409,640 & 14,804 & 6,342 & 5,721 & 7,497 & 62,133 & 437,409 \\
\hline Idaho* & 174,062 & 7,681 & 0 & 5,358 & 8,078 & 29,524 & 182,469 \\
\hline Illinois & $3,640,282$ & 248,477 & 43,279 & 84,224 & 113,659 & 363,784 & $3,514,427$ \\
\hline Indiana & $1,918,337$ & 61,860 & 6,173 & 15,133 & 38,661 & 140,019 & $1,936,529$ \\
\hline lowa* & 919,753 & 16,952 & 0 & 8,243 & 40,243 & 55,139 & 909,454 \\
\hline Kansas & 374,529 & 18,418 & 2,513 & 13,414 & 12,686 & 59,661 & 387,159 \\
\hline Kentucky & 550,206 & 17,923 & 2,414 & 2,166 & 13,362 & 69,298 & 583,639 \\
\hline Louisiana & $1,958,063$ & 58,455 & 2,704 & 18,434 & 58,298 & 82,836 & $1,903,008$ \\
\hline Maine* & 254,412 & 0 & 0 & 0 & 3,406 & 22,420 & 273,426 \\
\hline Maryland & $1,412,354$ & 139,203 & 13,061 & 43,565 & 24,395 & 134,132 & $1,326,262$ \\
\hline Massachusetts & $1,578,529$ & 174,926 & 37,227 & 64,940 & 33,763 & 197,911 & $1,465,584$ \\
\hline Michigan & $2,202,782$ & 175,885 & 26,757 & 88,584 & 32,918 & 266,476 & $2,145,114$ \\
\hline Minnesota & $1,469,670$ & 76,253 & 9,862 & 64,643 & 32,389 & 130,020 & $1,416,543$ \\
\hline Mississippi* & $1,394,046$ & 0 & 0 & 6,349 & 9,833 & 33,616 & $1,411,480$ \\
\hline Missouri & $1,803,947$ & 167,428 & 10,972 & 33,660 & 34,685 & 140,864 & $1,698,066$ \\
\hline Montana & 39,485 & 989 & 0 & 0 & 0 & 21,890 & 60,386 \\
\hline Nebraska* ${ }^{*}$ & 258,637 & 0 & 0 & 5,296 & 12,895 & 36,434 & 276,880 \\
\hline Nevada & $1,667,501$ & 17,156 & 15,407 & 112,333 & 17,964 & 56,156 & $1,560,797$ \\
\hline New Hampshire & 365,047 & 4,278 & 732 & 11,794 & 13,148 & 25,343 & 360,438 \\
\hline New Jersey & $1,981,156$ & 158,214 & 61,360 & 114,385 & 36,621 & 248,995 & $1,859,571$ \\
\hline New Mexico & 520,434 & 7,390 & 3,480 & 24,835 & 4,461 & 55,521 & 535,789 \\
\hline New York & $7,028,973$ & 550,098 & 624,138 & 931,438 & 71,516 & 634,590 & $5,486,373$ \\
\hline North Carolina & $1,632,553$ & 171,739 & 16,597 & 26,264 & 37,340 & 150,820 & $1,531,433$ \\
\hline North Dakota* & 164,293 & 0 & 0 & 0 & 3,916 & 14,222 & 174,599 \\
\hline Ohio & $2,308,609$ & 115,562 & 111,885 & 36,880 & 73,541 & 247,631 & $2,218,372$ \\
\hline Oklahoma & 531,352 & 12,280 & 3,077 & 5,770 & 13,616 & 64,315 & 560,924 \\
\hline Oregon & 834,870 & 41,318 & 9,843 & 19,460 & 19,216 & 108,408 & 853,441 \\
\hline Pennsylvania & $2,439,343$ & 152,554 & 17,388 & 47,998 & 94,699 & 248,397 & $2,375,101$ \\
\hline Rhode Island* & 234,849 & 0 & 0 & 6,457 & 8,299 & 19,769 & 239,862 \\
\hline South Carolina & $1,107,083$ & 20,293 & 2,107 & 10,094 & 181,216 & 65,870 & 959,243 \\
\hline South Dakota* & 299,166 & 2,711 & 0 & 1,443 & 19,934 & 13,544 & 288,622 \\
\hline Tennessee & $1,228,665$ & 96,010 & 59,006 & 40,429 & 26,356 & 110,836 & $1,117,700$ \\
\hline Texas & $3,743,828$ & 319,033 & 62,745 & 127,671 & 127,336 & 609,928 & $3,716,971$ \\
\hline Utah" & 412,404 & 50,745 & 0 & 19,581 & 10,355 & 72,073 & 403,796 \\
\hline Vermont & 226,937 & 4,126 & 2,114 & 7,004 & 2,647 & 16,111 & 227,157 \\
\hline Virginia & $1,397,864$ & 71,123 & 19,756 & 62,613 & 38,248 & 175,456 & $1,381,580$ \\
\hline Washington & $1,620,069$ & 26,325 & 20,628 & 55,076 & 37,348 & 183,552 & $1,664,244$ \\
\hline West Virginia & 273,339 & 4,038 & 0 & 485 & 18,655 & 22,991 & 273,152 \\
\hline Wisconsin & $1,327,454$ & 47,281 & 4,990 & 16,953 & 60,857 & 112,930 & $1,310,303$ \\
\hline Wyoming* & 26,261 & 0 & 0 & 0 & 0 & 11,142 & 37,403 \\
\hline$\overline{~ U . S . ~ T o t a l ~}$ & $\overline{855,088,464}$ & (5,045,093 & 2,409,918 & "6,361,000 & 1,705,888 & 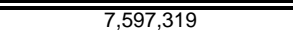 & $\overline{777,163,884}$ \\
\hline
\end{tabular}


Selective Sales--Adjustments to Amusements continued

\begin{tabular}{|c|c|c|c|c|}
\hline State & $\begin{array}{l}\text { Employmnet } \\
\text { Change } \\
\text { (percent) }\end{array}$ & $\begin{array}{c}\text { Adjusted Tax Base } \\
\text { (millions) }\end{array}$ & $\begin{array}{l}\text { Amusements State and Local Revenue } \\
\text { Collected } 1999 \text { total } \\
\text { (millions) }\end{array}$ & $\begin{array}{c}\text { RTS Revenue } \\
\text { (millions) }\end{array}$ \\
\hline Alabama & 17 & 515 & 0 & 19 \\
\hline Alaska & 7 & 199 & 2 & 7 \\
\hline Arizona & 33 & 2,686 & 1 & 99 \\
\hline Arkansas & 14 & 288 & 0 & 11 \\
\hline California & 11 & 12,756 & 0 & 472 \\
\hline Colorado & 35 & 2,509 & 73 & 93 \\
\hline Connecticut & 14 & 2,743 & 291 & 102 \\
\hline Delaware* & 18 & 299 & 0 & 11 \\
\hline D.C. & 65 & 136 & 0 & 5 \\
\hline Florida & 15 & 8,686 & 0 & 321 \\
\hline Georgia & 20 & 1,642 & 0 & 61 \\
\hline Hawaii & -6 & 411 & 0 & 15 \\
\hline Idaho* & 1 & 184 & 0 & 7 \\
\hline Illinois & 7 & 3,749 & 375 & 139 \\
\hline Indiana & 29 & 2,507 & 406 & 93 \\
\hline lowa* & 11 & 1,011 & 154 & 37 \\
\hline Kansas & 22 & 471 & 1 & 17 \\
\hline Kentucky & 17 & 685 & 0 & 25 \\
\hline Louisiana & 18 & 2,253 & 462 & 83 \\
\hline Maine ${ }^{*}$ & 18 & 322 & 0 & 12 \\
\hline Maryland & 4 & 1,384 & 10 & 51 \\
\hline Massachusetts & 20 & 1,756 & 7 & 65 \\
\hline Michigan & 24 & 2,649 & 6 & 98 \\
\hline Minnesota & 8 & 1,530 & 63 & 57 \\
\hline Mississippi* & 3 & 1,453 & 187 & 54 \\
\hline Missouri & 7 & 1,818 & 165 & 67 \\
\hline Montana & -1 & 60 & 38 & $\frac{1}{2}$ \\
\hline Nebraska* & 7 & 297 & 6 & 11 \\
\hline Nevada & 4 & 1,618 & 634 & 60 \\
\hline New Hampshire & 15 & 413 & 2 & 15 \\
\hline New Jersey & 7 & 1,987 & 326 & 74 \\
\hline New Mexico & 32 & 705 & 3 & 26 \\
\hline New York & 19 & 6,535 & 1 & 242 \\
\hline North Carolina & 32 & 2,018 & 0 & 75 \\
\hline North Dakota* & 7 & 187 & 11 & 7 \\
\hline Ohio & 23 & 2,718 & 0 & 101 \\
\hline Oklahoma & 24 & 694 & 8 & 26 \\
\hline Oregon & 19 & 1,013 & 0 & 37 \\
\hline Pennsylvania & 15 & 2,730 & 1 & 101 \\
\hline Rhode Island* & 9 & 261 & 0 & 10 \\
\hline South Carolina & 31 & 1,252 & 35 & 46 \\
\hline South Dakota* & 14 & 329 & 0 & 12 \\
\hline Tennessee & 17 & 1,312 & 0 & 49 \\
\hline Texas & 19 & 4,410 & 23 & 163 \\
\hline $\mathrm{Utah}^{\star}$ & 17 & 473 & 0 & 18 \\
\hline Vermont & 20 & 272 & 0 & 10 \\
\hline Virginia & 14 & 1,578 & 0 & 58 \\
\hline Washington & 16 & 1,923 & 0 & 71 \\
\hline West Virginia & 26 & 345 & 0 & 13 \\
\hline Wisconsin & 25 & 1,636 & 1 & 61 \\
\hline Wyoming* & 19 & 44 & 0 & 2 \\
\hline U.S. Total & 15 & 88,962 & 3,292 & 3,292 \\
\hline
\end{tabular}

*\$0 Receipts are actually undisclosed information due to the small number of firms participating in business. (Except for Wyoming, where all $\$ 0$ amounts

are accurate except for Promoters of Performing Arts, Sports and Similar Events, which is undisclosed) 
License Taxes--Total

\begin{tabular}{|c|c|c|c|c|c|c|c|c|}
\hline State & 1999 State Population & $\begin{array}{c}1999 \text { Total License Tax } \\
\text { Base } \\
\text { (\$ millions) }\end{array}$ & $\begin{array}{c}1999 \text { Tax } \\
\text { Capacity } \\
\text { (\$ millions) }\end{array}$ & $\begin{array}{c}1999 \text { Capacity per } \\
\text { capita } \\
\text { (\$/person) }\end{array}$ & $\begin{array}{l}1999 \text { Per Capita Capacity } \\
\text { Index }\end{array}$ & $\begin{array}{c}1999 \text { Tax Revenue } \\
\text { (\$ millions) }\end{array}$ & $\begin{array}{c}1999 \text { Revenue per } \\
\text { capita } \\
\text { (\$/person) }\end{array}$ & $\begin{array}{l}1999 \text { Tax Effort } \\
\text { Index }\end{array}$ \\
\hline Alabama & $4,369,862$ & $\mathrm{n} / \mathrm{a}$ & 415.4 & 95.06 & 107 & 357.44 & 81.80 & 86 \\
\hline Alaska & 619,500 & $\mathrm{n} / \mathrm{a}$ & 95.5 & 154.14 & 174 & 67.01 & 108.16 & 70 \\
\hline Arizona & $4,778,332$ & $\mathrm{n} / \mathrm{a}$ & 389.7 & 81.56 & 92 & 179.40 & 37.55 & 46 \\
\hline Arkansas & $2,551,373$ & n/a & 255.7 & 100.23 & 113 & 145.33 & 56.96 & 57 \\
\hline California & $33,145,121$ & $\mathrm{n} / \mathrm{a}$ & $2,580.5$ & 77.85 & 88 & $1,896.69$ & 57.22 & 74 \\
\hline Colorado & $4,056,133$ & $\mathrm{n} / \mathrm{a}$ & 420.5 & 103.66 & 117 & 267.82 & 66.03 & 64 \\
\hline Connecticut & $3,282,031$ & $\mathrm{n} / \mathrm{a}$ & 278.5 & 84.87 & 96 & 271.48 & 82.72 & 97 \\
\hline Delaware & 753,538 & n/a & 63.5 & 84.20 & 95 & 491.05 & 651.66 & 774 \\
\hline D.C. & 519,000 & $n / a$ & 29.7 & 57.14 & 64 & 23.12 & 44.54 & 78 \\
\hline Florida & $15,111,244$ & $\mathrm{n} / \mathrm{a}$ & $1,337.2$ & 88.49 & 100 & $1,176.66$ & 77.87 & 88 \\
\hline Georgia & $7,788,240$ & $\mathrm{n} / \mathrm{a}$ & 718.0 & 92.19 & 104 & 311.67 & 40.02 & 43 \\
\hline Hawaii & $1,185,497$ & $\mathrm{n} / \mathrm{a}$ & 76.4 & 64.48 & 73 & 109.46 & 92.34 & 143 \\
\hline Idaho & $1,251,700$ & $\mathrm{n} / \mathrm{a}$ & 175.5 & 140.20 & 158 & 138.16 & 110.38 & 79 \\
\hline Illinois & $12,128,370$ & n/a & 975.9 & 80.47 & 91 & $1,094.64$ & 90.25 & 112 \\
\hline Indiana & $5,942,901$ & n/a & 547.3 & 92.09 & 104 & 160.52 & 27.01 & 29 \\
\hline lowa & $2,869,413$ & $n / a$ & 325.7 & 113.49 & 128 & 386.76 & 134.79 & 119 \\
\hline Kansas & $2,654,052$ & n/a & 247.8 & 93.36 & 105 & 198.80 & 74.90 & 80 \\
\hline Kentucky & $3,960,825$ & $\mathrm{n} / \mathrm{a}$ & 344.9 & 87.07 & 98 & 408.49 & 103.13 & 118 \\
\hline Louisiana & $4,372,035$ & $\mathrm{n} / \mathrm{a}$ & 398.9 & 91.25 & 103 & 430.79 & 98.53 & 108 \\
\hline Maine & $1,253,040$ & $\mathrm{n} / \mathrm{a}$ & 119.2 & 95.17 & 107 & 102.78 & 82.03 & 86 \\
\hline Maryland & $5,171,634$ & $n / a$ & 406.2 & 78.55 & 89 & 220.06 & 42.55 & 54 \\
\hline Massachusetts & $6,175,169$ & $n / a$ & 528.7 & 85.61 & 97 & 322.04 & 52.15 & 61 \\
\hline Michigan & $9,863,775$ & $n / a$ & 970.5 & 98.39 & 111 & 874.92 & 88.70 & 90 \\
\hline Minnesota & $4,775,508$ & $\mathrm{n} / \mathrm{a}$ & 493.3 & 103.31 & 117 & 673.05 & 140.94 & 136 \\
\hline Mississippi & $2,768,619$ & $n / a$ & 246.7 & 89.10 & 101 & 215.28 & 77.76 & 87 \\
\hline Missouri & $5,468,338$ & $n / a$ & 554.8 & 101.45 & 114 & 417.27 & 76.31 & 75 \\
\hline Montana & 882,779 & $\mathrm{n} / \mathrm{a}$ & 150.7 & 170.71 & 193 & 99.13 & 112.29 & 66 \\
\hline Nebraska & $1,666,028$ & $n / a$ & 183.3 & 110.04 & 124 & 120.32 & 72.22 & 66 \\
\hline Nevada & $1,809,253$ & $n / a$ & 147.0 & 81.22 & 92 & 148.60 & 82.13 & 101 \\
\hline New Hampshire & $1,201,134$ & n/a & 124.0 & 103.25 & 117 & 80.66 & 67.15 & 65 \\
\hline New Jersey & $8,143,412$ & $\mathrm{n} / \mathrm{a}$ & 621.6 & 76.33 & 86 & 555.04 & 68.16 & 89 \\
\hline New Mexico & $1,739,844$ & $\mathrm{n} / \mathrm{a}$ & 169.1 & 97.20 & 110 & 157.17 & 90.34 & 93 \\
\hline New York & $18,196,601$ & $n / a$ & $1,234.1$ & 67.82 & 77 & 892.53 & 49.05 & 72 \\
\hline North Carolina & $7,650,789$ & $\mathrm{n} / \mathrm{a}$ & 639.3 & 83.56 & 94 & 755.30 & 98.72 & 118 \\
\hline North Dakota & 633,666 & $\mathrm{n} / \mathrm{a}$ & 89.7 & 141.57 & 160 & 49.76 & 78.53 & 55 \\
\hline Ohio & $11,256,654$ & $n / a$ & $1,053.8$ & 93.61 & 106 & $1,150.90$ & 102.24 & 109 \\
\hline Oklahoma & $3,358,044$ & $n / a$ & 310.9 & 92.59 & 104 & 639.92 & 190.56 & 206 \\
\hline Oregon & $3,316,154$ & $\mathrm{n} / \mathrm{a}$ & 403.1 & 121.57 & 137 & 397.95 & 120.00 & 99 \\
\hline Pennsylvania & $11,994,016$ & $\mathrm{n} / \mathrm{a}$ & $1,116.7$ & 93.10 & 105 & $1,949.83$ & 162.57 & 175 \\
\hline Rhode Island & 990,819 & $\mathrm{n} / \mathrm{a}$ & 78.2 & 78.91 & 89 & 65.93 & 66.55 & 84 \\
\hline South Carolina & $3,885,736$ & $\mathrm{n} / \mathrm{a}$ & 336.7 & 86.66 & 98 & 177.55 & 45.69 & 53 \\
\hline South Dakota & 733,133 & $\mathrm{n} / \mathrm{a}$ & 106.7 & 145.57 & 164 & 66.42 & 90.60 & 62 \\
\hline Tennessee & $5,483,535$ & $n / a$ & 541.1 & 98.67 & 111 & 793.10 & 144.63 & 147 \\
\hline Texas & $20,044,141$ & $n / a$ & $1,536.7$ & 76.66 & 87 & $3,419.71$ & 170.61 & 223 \\
\hline Utah & $2,129,836$ & $\mathrm{n} / \mathrm{a}$ & 215.2 & 101.02 & 114 & 104.83 & 49.22 & 49 \\
\hline Vermont & 593,740 & $n / a$ & 69.3 & 116.70 & 132 & 44.94 & 75.69 & 65 \\
\hline Virginia & $6,872,912$ & $\mathrm{n} / \mathrm{a}$ & 623.1 & 90.65 & 102 & 475.00 & 69.11 & 76 \\
\hline Washington & $5,756,361$ & $n / a$ & 553.4 & 96.14 & 108 & 459.82 & 79.88 & 83 \\
\hline West Virginia & $1,806,928$ & $\mathrm{n} / \mathrm{a}$ & 162.9 & 90.18 & 102 & 109.40 & 60.55 & 67 \\
\hline Wisconsin & $5,250,446$ & $n / a$ & 624.4 & 118.92 & 134 & 422.77 & 80.52 & 68 \\
\hline Wyoming & 479,602 & $n / a$ & 75.7 & 157.91 & 178 & 86.91 & 181.22 & 115 \\
\hline $\begin{array}{l}\text { Totals } \\
\end{array}$ & $272,690,813$ & $\overline{n-a}$ & $24,164.2$ & 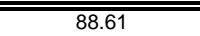 & 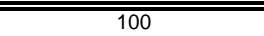 & 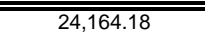 & ב88.61 & 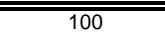 \\
\hline
\end{tabular}




\begin{tabular}{|c|c|c|c|c|c|c|c|c|}
\hline State & 1999 State Population & $\begin{array}{c}1999 \text { Total Motor Vehicles } \\
\text { Registration Tax Base } \\
\text { (millions) }\end{array}$ & $\begin{array}{l}1999 \text { Tax } \\
\text { Capacity } \\
\text { (\$ millions) }\end{array}$ & $\begin{array}{c}1999 \text { Capacity per } \\
\text { capita } \\
\text { (\$/person) }\end{array}$ & $\begin{array}{l}1999 \text { Per Capita Capacity } \\
\text { Index }\end{array}$ & $\begin{array}{c}1999 \text { Tax } \\
\text { Revenue } \\
\text { (\$ millions) }\end{array}$ & $\begin{array}{c}1999 \text { Revenue per } \\
\text { capita } \\
\text { (\$/person) }\end{array}$ & $\begin{array}{l}1999 \text { Tax Effort } \\
\text { Index }\end{array}$ \\
\hline Alabama & $4,369,862$ & 3.91 & 279.88 & 64.05 & 114 & 204.72 & 46.85 & 73 \\
\hline Alaska & 619,500 & 0.56 & 40.26 & 64.99 & 116 & 43.95 & 70.94 & 109 \\
\hline Arizona & $4,778,332$ & 3.34 & 238.99 & 50.02 & 89 & 144.06 & 30.15 & 60 \\
\hline Arkansas & $2,551,373$ & 1.78 & 127.66 & 50.03 & 89 & 106.72 & 41.83 & 84 \\
\hline California & $33,145,121$ & 25.92 & $1,857.19$ & 56.03 & 100 & $1,662.75$ & 50.17 & 90 \\
\hline Colorado & $4,056,133$ & 3.72 & 266.40 & 65.68 & 117 & 197.42 & 48.67 & 74 \\
\hline Connecticut & $3,282,031$ & 2.75 & 196.79 & 59.96 & 107 & 225.33 & 68.66 & 115 \\
\hline Delaware & 753,538 & 0.62 & 44.08 & 58.50 & 104 & 30.29 & 40.19 & 69 \\
\hline D.C. & 519,000 & 0.22 & 15.90 & 30.63 & 54 & 17.93 & 34.55 & 113 \\
\hline Florida & $15,111,244$ & 11.28 & 807.94 & 53.47 & 95 & 910.36 & 60.24 & 113 \\
\hline Georgia & $7,788,240$ & 6.92 & 495.78 & 63.66 & 113 & 217.90 & 27.98 & 44 \\
\hline Hawaii & $1,185,497$ & 0.72 & 51.35 & 43.32 & 77 & 106.35 & 89.71 & 207 \\
\hline Idaho & $1,251,700$ & 1.14 & 81.38 & 65.01 & 116 & 107.81 & 86.13 & 132 \\
\hline Illinois & $12,128,370$ & 9.46 & 677.85 & 55.89 & 99 & 893.31 & 73.65 & 132 \\
\hline Indiana & $5,942,901$ & 5.46 & 391.13 & 65.81 & 117 & 141.54 & 23.82 & 36 \\
\hline lowa & $2,869,413$ & 3.13 & 224.41 & 78.21 & 139 & 325.23 & 113.34 & 145 \\
\hline Kansas & $2,654,052$ & 2.19 & 157.12 & 59.20 & 105 & 146.96 & 55.37 & 94 \\
\hline Kentucky & $3,960,825$ & 2.75 & 197.30 & 49.81 & 89 & 211.59 & 53.42 & 107 \\
\hline Louisiana & $4,372,035$ & 3.44 & 246.74 & 56.44 & 100 & 107.32 & 24.55 & 43 \\
\hline Maine & $1,253,040$ & 0.93 & 66.89 & 53.38 & 95 & 82.27 & 65.65 & 123 \\
\hline Maryland & $5,171,634$ & 3.83 & 274.16 & 53.01 & 94 & 177.56 & 34.33 & 65 \\
\hline Massachusetts & $6,175,169$ & 5.29 & 379.26 & 61.42 & 109 & 234.12 & 37.91 & 62 \\
\hline Michigan & $9,863,775$ & 8.23 & 589.76 & 59.79 & 106 & 771.70 & 78.24 & 131 \\
\hline Minnesota & $4,775,508$ & 4.18 & 299.52 & 62.72 & 111 & 596.70 & 124.95 & 199 \\
\hline Mississippi & $2,768,619$ & 2.28 & 163.43 & 59.03 & 105 & 109.47 & 39.54 & 67 \\
\hline Missouri & $5,468,338$ & 4.41 & 316.13 & 57.81 & 103 & 264.22 & 48.32 & 84 \\
\hline Montana & 882,779 & 0.99 & 70.98 & 80.40 & 143 & 63.92 & 72.40 & 90 \\
\hline Nebraska & $1,666,028$ & 1.53 & 109.79 & 65.90 & 117 & 94.35 & 56.63 & 86 \\
\hline Nevada & $1,809,253$ & 1.19 & 85.29 & 47.14 & 84 & 111.19 & 61.46 & 130 \\
\hline New Hampshire & $1,201,134$ & 1.08 & 77.08 & 64.17 & 114 & 61.38 & 51.10 & 80 \\
\hline New Jersey & $8,143,412$ & 5.90 & 422.45 & 51.88 & 92 & 363.60 & 44.65 & 86 \\
\hline New Mexico & $1,739,844$ & 1.58 & 113.24 & 65.09 & 116 & 132.61 & 76.22 & 117 \\
\hline New York & $18,196,601$ & 10.53 & 754.54 & 41.47 & 74 & 702.41 & 38.60 & 93 \\
\hline North Carolina & $7,650,789$ & 5.76 & 412.43 & 53.91 & 96 & 425.46 & 55.61 & 103 \\
\hline North Dakota & 633,666 & 0.69 & 49.46 & 78.05 & 139 & 39.83 & 62.86 & 81 \\
\hline Ohio & $11,256,654$ & 10.23 & 733.22 & 65.14 & 116 & 685.40 & 60.89 & 93 \\
\hline Oklahoma & $3,358,044$ & 2.91 & 208.35 & 62.04 & 110 & 579.53 & 172.58 & 278 \\
\hline Oregon & $3,316,154$ & 3.00 & 214.68 & 64.74 & 115 & 340.83 & 102.78 & 159 \\
\hline Pennsylvania & $11,994,016$ & 9.07 & 650.17 & 54.21 & 96 & 735.84 & 61.35 & 113 \\
\hline Rhode Island & 990,819 & 0.74 & 53.09 & 53.59 & 95 & 52.58 & 53.06 & 99 \\
\hline South Carolina & $3,885,736$ & 2.96 & 211.86 & 54.52 & 97 & 113.04 & 29.09 & 53 \\
\hline South Dakota & 733,133 & 0.78 & 55.97 & 76.34 & 136 & 48.93 & 66.74 & 87 \\
\hline Tennessee & $5,483,535$ & 4.42 & 316.44 & 57.71 & 103 & 297.35 & 54.23 & 94 \\
\hline Texas & $20,044,141$ & 13.31 & 953.72 & 47.58 & 85 & $1,136.96$ & 56.72 & 119 \\
\hline Utah & $2,129,836$ & 1.55 & 111.29 & 52.25 & 93 & 78.15 & 36.69 & 70 \\
\hline Vermont & 593,740 & 0.51 & 36.73 & 61.86 & 110 & 33.65 & 56.67 & 92 \\
\hline Virginia & $6,872,912$ & 5.82 & 416.71 & 60.63 & 108 & 399.60 & 58.14 & 96 \\
\hline Washington & $5,756,361$ & 4.89 & 350.00 & 60.80 & 108 & 393.19 & 68.30 & 112 \\
\hline West Virginia & $1,806,928$ & 1.35 & 96.65 & 53.49 & 95 & 81.68 & 45.20 & 85 \\
\hline Wisconsin & $5,250,446$ & 4.35 & 311.84 & 59.39 & 106 & 276.68 & 52.70 & 89 \\
\hline Wyoming & 479,602 & 0.54 & 38.76 & 80.81 & 144 & 56.33 & 117.46 & 145 \\
\hline $\begin{array}{l}\text { Totals } \\
\end{array}$ & $\begin{array}{l}272,690,813 \\
\end{array}$ & $\overline{214.13}$ & $15,342.02$ & 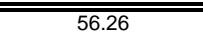 & 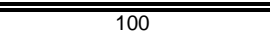 & 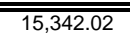 & $\bar{~} 56.26$ & 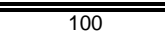 \\
\hline
\end{tabular}


1999 Total Motor Vehicles Operators Tax

\section{Base (equal to tota number of licensed}

1999 State Population

drivers)

\begin{tabular}{lccc} 
& 1999 State Population & $\begin{array}{c}\text { drivers) } \\
\text { (millions) }\end{array}$ & $\begin{array}{c}\text { 1999 } \\
\text { (\$ }\end{array}$ \\
\hline Alabama & $4,369,862$ & 3.44 & \\
Alaska & 619,500 & 0.46 & \\
Arizona & $4,778,332$ & 3.25 & \\
Arkansas & $2,551,373$ & 1.92 & \\
Can & $33,145,121$ & 20.66 &
\end{tabular}

$\begin{array}{ll}\text { Colorado } & 4,056,133 \\ \text { Connecticut } & 3,282,03 \\ & \end{array}$

$\begin{array}{ll}\text { Delaware } & 3,282,031 \\ \text { DC } & 753,538\end{array}$

D.C.

(53,538

Florid

Georgia

daho

Illinois

Indiana

Kansas

Kentucky

Louisiana

Maryland

Massachusetts

Michigan

Minnesota

Mississipp

Montana

Nevada

New Hampshire

New Jersey

New Mexico

North Carolina

North Dakota

Ohio
Oklahoma

Oregon

Pennsylvania

Rhode Island

South Carolina
South Dakota

Tennessee

Texas
Utah
Vermont
Virginia

Virginia

Washington

West Virginia

Wisconsin

Totals

RTS rate

519,000
$15,111,244$

$7,788,240$

$1,185,497$

2, 128,370

$5,942,901$

$2,654,052$

$2,654,052$
$3,960,825$

$4,372,035$

$\begin{array}{r}4,253,040 \\ \hline 5,171,634\end{array}$

$6,175,169$

$9,863,775$

$4,775,508$

$2,768,619$

882,779

$1,809,253$

$1,201,134$

$8,143,412$

(19,196,601

$7,650,789$

$11,256,654$
$3,358,044$

$3,358,044$

$1,994,01$

990,819

$3,885,736$

733,133
$5,483,535$

$20,044,141$

$2,129,836$

593,740
$6,872,912$

$5,756,361$

$1,806,928$

$5,250,446$

$272,690,813$

$\$ 6.94 /$ license

0.35
12.21

0.21
5.39

0.87
7.81
3.92

7.82
3.92
1.87

$1.87-13.47$

$\begin{array}{ll}1.65 & 12.98 \\ 2.75 & 18.38 \\ 0.91 & 6.33\end{array}$

2.75
0.91

0.91

$3.19 \quad 22.1$

$1.77 \quad 12.30$

$\begin{array}{ll}0.54 & 3.74 \\ 4.12 & 28.60\end{array}$

13.34

$\begin{array}{ll}4.70 & 32.59 \\ 4.10 & 28.46\end{array}$

$\begin{array}{cc}4.70 & 28.46 \\ 1.28 & 8.86 \\ .72 & 25.81\end{array}$

0.36

1999 Capacity per 1999 Per Capita Capacity Index

Revenue (\$ millions)

\begin{tabular}{ccc}
2.86 & 5.46 & \\
3.18 & 5.13 & 108 \\
22.52 & 4.71 & \\
13.33 & 5.22 & 100 \\
143.32 & 4.32 & 110 \\
\hline 2.59 & 5.08
\end{tabular}
$\begin{array}{ll}13.33 & 5.22 \\ 143.32 & 4.32 \\ 20.59 & 5.08\end{array}$

$\begin{array}{ll}20.59 & 5.08 \\ 16.38 & 4.99\end{array}$

\begin{tabular}{ll}
16.38 & 5.90 \\
3.81 & 5.05 \\
2.42 & 4.67 \\
\hline
\end{tabular}

2.42

84.71

4.61
5.41
6.02

5.02
54.19

$\begin{array}{lll}4.41 & 30.57 & 4.27 \\ 6.83 & 47.39 & 4.80\end{array}$

$\begin{array}{lll}6.83 & 47.39 & 4.80 \\ 2.89 & 20.03 & 4.19\end{array}$

\begin{tabular}{lll}
4.41 & 4.80 \\
2.89 & 20.03 & 4.19 \\
\hline
\end{tabular}

$\begin{array}{lll}3.82 & 26.49 & 4.84 \\ 0.65 & 4.53 & 5.13\end{array}$

$\begin{array}{llll}1.77 & 4.53 & 5.13 & 102 \\ 0.65 & 8.28 & 4.97 & 108 \\ 1.19 & 8.90 & 4.92 & 105\end{array}$

\begin{tabular}{lll}
0.15 & 4.28 & 4.97 \\
1.28 & 8.90 & 4.92 \\
0.91 & 0.33 & 5.27 \\
\hline
\end{tabular}

$\begin{array}{lll}0.33 & 5.27 \\ 0.91 & 6.93\end{array}$

$\begin{array}{cccc}1.28 & 4.73 & 100 \\ 1.21 & 38.54 & 4.83 & 102\end{array}$

$\begin{array}{lll}1.21 & 8.41 & 4.83 \\ 10.59 & 73.45 & 4.04\end{array}$

$\begin{array}{rrr}5.51 & 38.23 & 5.00 \\ 0.46 & 3.17 & 5.00\end{array}$

$\begin{array}{lll}0.46 & 3.17 & 5.00 \\ 7.99 & 5.00\end{array}$

$\begin{array}{lll} & 3.99 & 5.00 \\ 2.31 & 55.44 & 4.93\end{array}$

$\begin{array}{lll}2.31 & 16.01 & 4.77 \\ 2.44 & 16.92 & 5.10 \\ 8.44 & 5.55 & 4.88\end{array}$

\begin{tabular}{lll}
2.44 & 16.92 & 5.10 \\
8.44 & 58.55 & 4.88 \\
\hline
\end{tabular}

\begin{tabular}{rrr}
2.44 & 58.55 & 4.88 \\
8.44 & 4.75 & 4.80 \\
0.69 & 19.04 & 4.90 \\
\hline
\end{tabular}

\begin{tabular}{lcc}
8.64 & 4.75 & 4.80 \\
\hline 2.74 & 19.04 & 4.90 \\
0.54 & 3.74 & 5.10
\end{tabular}

$\begin{array}{lll}0.54 & 3.74 & 5.10 \\ 1.12 & 28.60 & 5.22 \\ 13.34 & 92.53 & 4.62\end{array}$

\begin{tabular}{ccc}
4.3 .34 & 92.53 & 4.62 \\
1.42 & 9.82 & 4.61 \\
\hline 0.50 & 3.44 & 5.80 \\
4.70 & 32.59 & 4.74
\end{tabular}

25.81

2.51

\begin{tabular}{lc}
4.62 & 98 \\
4.61 & 97 \\
\hline 5.80 & 123 \\
4.74 & 100 \\
4.94 & 105 \\
4.90 & 104
\end{tabular}

5.22

\begin{tabular}{|c|c|c|c|}
\hline & (\$ mintons) & (\$iperson) & \\
\hline 115 & 16.62 & 3.80 & 70 \\
\hline 108 & 0.00 & 0.00 & 0 \\
\hline 100 & 12.96 & 2.71 & 58 \\
\hline 110 & 7.46 & 2.92 & 56 \\
\hline 91 & 141.11 & 4.26 & 98 \\
\hline 107 & 11.94 & 2.94 & 58 \\
\hline 105 & 29.65 & 9.03 & 181 \\
\hline 107 & 0.14 & 0.19 & 4 \\
\hline 99 & 1.51 & 2.91 & 62 \\
\hline 118 & 113.04 & 7.48 & 133 \\
\hline 102 & 40.31 & 5.18 & 108 \\
\hline 93 & 0.33 & 0.28 & 6 \\
\hline 102 & 5.67 & 4.53 & 94 \\
\hline 94 & 59.34 & 4.89 & 110 \\
\hline 97 & 0.00 & 0.00 & 0 \\
\hline 99 & 10.62 & 3.70 & 79 \\
\hline 103 & 12.03 & 4.53 & 93 \\
\hline 98 & 6.63 & 1.67 & 36 \\
\hline 92 & 8.74 & 2.00 & 46 \\
\hline 107 & 5.54 & 4.42 & 88 \\
\hline 90 & 21.22 & 4.10 & 96 \\
\hline 105 & 59.31 & 9.60 & 194 \\
\hline 102 & 43.57 & 4.42 & 92 \\
\hline 89 & 27.62 & 5.78 & 138 \\
\hline 94 & 21.40 & 7.73 & 174 \\
\hline 102 & 21.49 & 3.93 & 81 \\
\hline 108 & 5.49 & 6.22 & 121 \\
\hline 105 & 8.32 & 4.99 & 100 \\
\hline 104 & 11.77 & 6.50 & 132 \\
\hline 111 & 8.27 & 6.88 & 131 \\
\hline 100 & 31.55 & 3.87 & 82 \\
\hline 102 & 7.40 & 4.25 & 88 \\
\hline 85 & 84.60 & 4.65 & 115 \\
\hline 106 & 81.80 & 10.69 & 214 \\
\hline 106 & 3.31 & 5.22 & 105 \\
\hline 104 & 39.22 & 3.48 & 71 \\
\hline 101 & 6.84 & 2.04 & 43 \\
\hline 108 & 20.44 & 6.16 & 121 \\
\hline 103 & 51.86 & 4.32 & 89 \\
\hline 101 & 0.41 & 0.42 & 9 \\
\hline 104 & 15.03 & 3.87 & 79 \\
\hline 108 & 1.35 & 1.84 & 36 \\
\hline 110 & 36.72 & 6.70 & 128 \\
\hline 98 & 98.55 & 4.92 & 107 \\
\hline 97 & 5.79 & 2.72 & 59 \\
\hline 123 & 3.75 & 6.32 & 109 \\
\hline 100 & 27.58 & 4.01 & 85 \\
\hline 105 & 27.65 & 4.80 & 97 \\
\hline 104 & 4.47 & 2.47 & 50 \\
\hline 104 & 27.33 & 5.21 & 106 \\
\hline 110 & 2.38 & 4.97 & 95 \\
\hline$\overline{100}$ & $\overline{1,290.11}$ & 4.73 & $\overline{100}$ \\
\hline
\end{tabular}

\begin{tabular}{l}
104 \\
110 \\
\hline 100
\end{tabular} 
License Taxes--Hunting and Fishing

\begin{tabular}{|c|c|c|c|c|c|c|c|c|c|c|}
\hline State & $\begin{array}{l}1999 \text { State } \\
\text { Population }\end{array}$ & $\begin{array}{c}1999 \text { Total Hunting } \\
\text { Licenses, Tags, Permits } \\
\text { and Stamps } \\
\text { (millions) }\end{array}$ & $\begin{array}{l}1999 \text { Total Fishing } \\
\text { Licenses, Tags, Permits } \\
\text { and Stamps } \\
\text { (millions) }\end{array}$ & $\begin{array}{c}1999 \text { Total Hunting and Fishing Tax Base } \\
\text { (equal to total number of licenses) } \\
\text { (millions) }\end{array}$ & $\begin{array}{l}1999 \text { Tax } \\
\text { Capacity } \\
\text { (\$ millions) }\end{array}$ & $\begin{array}{c}1999 \text { Capacity per } \\
\text { capita } \\
\text { (\$/person) }\end{array}$ & $\begin{array}{l}1999 \text { Per Capita } \\
\text { Capacity Index }\end{array}$ & $\begin{array}{l}1999 \text { Tax } \\
\text { Revenue } \\
\text { (\$ millions) }\end{array}$ & $\begin{array}{l}1999 \text { Revenue } \\
\text { per capita } \\
\text { (\$/person) }\end{array}$ & $\begin{array}{l}1999 \text { Tax Effort } \\
\text { Index }\end{array}$ \\
\hline Alabama & $4,369,862$ & 0.49 & 0.30 & 0.79 & 12.94 & 2.96 & 69 & 13.48 & 3.08 & 104 \\
\hline Alaska & 619,500 & 0.64 & 0.22 & 0.85 & 14.04 & 22.66 & 528 & 21.64 & 34.93 & 154 \\
\hline Arizona & $4,778,332$ & 0.66 & 0.36 & 1.02 & 16.77 & 3.51 & 82 & 15.28 & 3.20 & 91 \\
\hline Arkansas & $2,551,373$ & 0.75 & 0.54 & 1.30 & 21.32 & 8.36 & 195 & 19.72 & 7.73 & 92 \\
\hline California & $33,145,121$ & 3.04 & 0.84 & 3.88 & 63.76 & 1.92 & 45 & 60.01 & 1.81 & 94 \\
\hline Colorado & $4,056,133$ & 0.94 & 0.56 & 1.50 & 24.61 & 6.07 & 142 & 53.51 & 13.19 & 217 \\
\hline Connecticut & $3,282,031$ & 0.17 & 0.14 & 0.32 & 5.24 & 1.60 & 37 & 4.00 & 1.22 & 76 \\
\hline Delaware & 753,538 & 0.03 & 0.04 & 0.07 & 1.08 & 1.43 & 33 & 0.76 & 1.01 & 71 \\
\hline D.C. & 519,000 & 0.00 & 0.00 & 0.00 & 0.00 & 0.00 & 0 & 0.00 & 0.00 & 0 \\
\hline Florida & $15,111,244$ & 2.02 & 0.33 & 2.35 & 38.59 & 2.55 & 60 & 26.81 & 1.77 & 69 \\
\hline Georgia & $7,788,240$ & 0.80 & 0.89 & 1.68 & 27.68 & 3.55 & 83 & 18.88 & 2.42 & 68 \\
\hline Hawaii & $1,185,497$ & 0.01 & 0.01 & 0.02 & 0.26 & 0.22 & 5 & 0.18 & 0.15 & 68 \\
\hline Idaho & $1,251,700$ & 0.55 & 0.88 & 1.43 & 23.59 & 18.84 & 439 & 23.54 & 18.80 & 100 \\
\hline Illinois & $12,128,370$ & 0.84 & 0.88 & 1.71 & 28.19 & 2.32 & 54 & 20.64 & 1.70 & 73 \\
\hline Indiana & $5,942,901$ & 0.67 & 0.65 & 1.32 & 21.67 & 3.65 & 85 & 14.15 & 2.38 & 65 \\
\hline lowa & $2,869,413$ & 0.43 & 0.86 & 1.30 & 21.31 & 7.43 & 173 & 18.03 & 6.28 & 85 \\
\hline Kansas & $2,654,052$ & 0.33 & 0.45 & 0.78 & 12.78 & 4.81 & 112 & 14.99 & 5.65 & 117 \\
\hline Kentucky & $3,960,825$ & 0.60 & 0.74 & 1.34 & 21.97 & 5.55 & 129 & 16.79 & 4.24 & 76 \\
\hline Louisiana & $4,372,035$ & 0.97 & 0.62 & 1.59 & 26.15 & 5.98 & 139 & 14.11 & 3.23 & 54 \\
\hline Maine & $1,253,040$ & 0.27 & 0.21 & 0.48 & 7.89 & 6.29 & 147 & 11.86 & 9.46 & 150 \\
\hline Maryland & $5,171,634$ & 0.67 & 0.22 & 0.89 & 14.61 & 2.82 & 66 & 9.91 & 1.92 & 68 \\
\hline Massachusetts & $6,175,169$ & 0.35 & 0.24 & 0.59 & 9.76 & 1.58 & 37 & 6.92 & 1.12 & 71 \\
\hline Michigan & $9,863,775$ & 1.46 & 2.43 & 3.89 & 63.99 & 6.49 & 151 & 47.67 & 4.83 & 74 \\
\hline Minnesota & $4,775,508$ & 1.39 & 1.17 & 2.56 & 42.09 & 8.81 & 206 & 44.61 & 9.34 & 106 \\
\hline Mississippi & $2,768,619$ & 0.49 & 0.29 & 0.77 & 12.74 & 4.60 & 107 & 12.71 & 4.59 & 100 \\
\hline Missouri & $5,468,338$ & 1.49 & 1.12 & 2.62 & 43.02 & 7.87 & 183 & 29.46 & 5.39 & 68 \\
\hline Montana & 882,779 & 0.58 & 0.72 & 1.31 & 21.48 & 24.33 & 567 & 28.64 & 32.44 & 133 \\
\hline Nebraska & $1,666,028$ & 0.43 & 0.42 & 0.85 & 14.04 & 8.43 & 197 & 11.72 & 7.03 & 83 \\
\hline Nevada & $1,809,253$ & 0.28 & 0.10 & 0.38 & 6.22 & 3.44 & 80 & 6.18 & 3.42 & 99 \\
\hline New Hampshire & $1,201,134$ & 0.17 & 0.26 & 0.43 & 7.10 & 5.91 & 138 & 6.87 & 5.72 & 97 \\
\hline New Jersey & $8,143,412$ & 0.29 & 0.28 & 0.57 & 9.35 & 1.15 & 27 & 10.46 & 1.28 & 112 \\
\hline New Mexico & $1,739,844$ & 0.28 & 0.24 & 0.52 & 8.50 & 4.88 & 114 & 14.88 & 8.55 & 175 \\
\hline New York & $18,196,601$ & 1.05 & 1.67 & 2.72 & 44.78 & 2.46 & 57 & 32.40 & 1.78 & 72 \\
\hline North Carolina & $7,650,789$ & 0.70 & 0.50 & 1.21 & 19.82 & 2.59 & 60 & 21.04 & 2.75 & 106 \\
\hline North Dakota & 633,666 & 0.16 & 0.45 & 0.61 & 10.06 & 15.87 & 370 & 6.62 & 10.45 & 66 \\
\hline Ohio & $11,256,654$ & 1.17 & 1.21 & 2.37 & 39.04 & 3.47 & 81 & 31.83 & 2.83 & 82 \\
\hline Oklahoma & $3,358,044$ & 0.53 & 0.32 & 0.85 & 14.05 & 4.18 & 98 & 15.02 & 4.47 & 107 \\
\hline Oregon & $3,316,154$ & 0.99 & 1.46 & 2.45 & 40.28 & 12.15 & 283 & 30.71 & 9.26 & 76 \\
\hline Pennsylvania & $11,994,016$ & 1.80 & 2.52 & 4.32 & 71.10 & 5.93 & 138 & 45.80 & 3.82 & 64 \\
\hline Rhode Island & 990,819 & 0.06 & 0.03 & 0.09 & 1.50 & 1.51 & 35 & 0.72 & 0.73 & 48 \\
\hline South Carolina & $3,885,736$ & 0.60 & 0.27 & 0.87 & 14.32 & 3.69 & 86 & 12.32 & 3.17 & 86 \\
\hline South Dakota & 733,133 & 0.36 & 0.41 & 0.77 & 12.68 & 17.29 & 403 & 14.25 & 19.44 & 112 \\
\hline Tennessee & $5,483,535$ & 1.22 & 0.84 & 2.07 & 34.01 & 6.20 & 145 & 135.66 & 24.74 & 399 \\
\hline Texas & $20,044,141$ & 2.10 & 1.24 & 3.34 & 54.92 & 2.74 & 64 & 56.44 & 2.82 & 103 \\
\hline Utah & $2,129,836$ & 0.84 & 0.39 & 1.23 & 20.27 & 9.52 & 222 & 19.37 & 9.09 & 96 \\
\hline Vermont & 593,740 & 0.13 & 0.21 & 0.34 & 5.62 & 9.46 & 221 & 6.26 & 10.54 & 111 \\
\hline Virginia & $6,872,912$ & 0.81 & 0.83 & 1.64 & 27.03 & 3.93 & 92 & 20.29 & 2.95 & 75 \\
\hline Washington & $5,756,361$ & 1.08 & 0.74 & 1.82 & 29.93 & 5.20 & 121 & 25.81 & 4.48 & 86 \\
\hline West Virginia & $1,806,928$ & 0.28 & 0.28 & 0.57 & 9.30 & 5.15 & 120 & 15.36 & 8.50 & 165 \\
\hline Wisconsin & $5,250,446$ & 1.52 & 2.63 & 4.15 & 68.26 & 13.00 & 303 & 58.84 & 11.21 & 86 \\
\hline Wyoming & 479,602 & 0.34 & 0.24 & 0.58 & 9.53 & 19.88 & 464 & 22.09 & 46.07 & 232 \\
\hline $\begin{array}{l}\text { Totals } \\
\end{array}$ & $272,690,813$ & 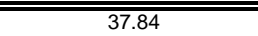 & $\overline{333.26}$ & $\overline{771.10}$ & 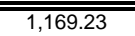 & 4.29 & 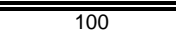 & 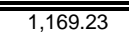 & 4.29 & 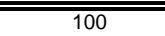 \\
\hline
\end{tabular}


License Taxes--Corporate Licenses

\begin{tabular}{|c|c|c|c|c|c|c|c|c|}
\hline State & 1999 State Population & $\begin{array}{l}1999 \text { Total Corporate } \\
\text { Licenses } \\
\text { (thousands) }\end{array}$ & $\begin{array}{l}1999 \text { Tax } \\
\text { Capacity } \\
\text { (\$ millions) }\end{array}$ & $\begin{array}{l}1999 \text { Capacity } \\
\text { per capita } \\
\text { (\$/person) }\end{array}$ & $\begin{array}{l}1999 \text { Per Capita } \\
\text { Capacity Index }\end{array}$ & $\begin{array}{c}1999 \text { Tax } \\
\text { Revenue } \\
\text { (\$ millions) }\end{array}$ & $\begin{array}{l}1999 \text { Revenue } \\
\text { per capita } \\
\text { (\$/person) }\end{array}$ & $\begin{array}{l}1999 \text { Tax Effort } \\
\text { Index }\end{array}$ \\
\hline Alabama & $4,369,862$ & 60.52 & 71.27 & 16.31 & 70 & 122.62 & 28.06 & 172 \\
\hline Alaska & 619,500 & 10.11 & 11.90 & 19.21 & 82 & 1.42 & 2.30 & 12 \\
\hline Arizona & $4,778,332$ & 83.98 & 98.90 & 20.70 & 89 & 7.11 & 1.49 & 7 \\
\hline Arkansas & $2,551,373$ & 44.02 & 51.84 & 20.32 & 87 & 11.44 & 4.48 & 22 \\
\hline California & $33,145,121$ & 438.38 & 516.26 & 15.58 & 67 & 32.82 & 0.99 & 6 \\
\hline Colorado & $4,056,133$ & 110.44 & 130.06 & 32.06 & 137 & 4.94 & 1.22 & 4 \\
\hline Connecticut & $3,282,031$ & 67.93 & 80.00 & 24.38 & 104 & 12.50 & 3.81 & 16 \\
\hline Delaware & 753,538 & 21.86 & 25.74 & 34.16 & 146 & 459.86 & 610.27 & 1,787 \\
\hline D.C. & 519,000 & 13.75 & 16.20 & 31.21 & 134 & 3.68 & 7.08 & 23 \\
\hline Florida & $15,111,244$ & 539.80 & 635.71 & 42.07 & 180 & 126.46 & 8.37 & 20 \\
\hline Georgia & $7,788,240$ & 166.56 & 196.15 & 25.19 & 108 & 34.59 & 4.44 & 18 \\
\hline Hawaii & $1,185,497$ & 25.64 & 30.20 & 25.47 & 109 & 2.61 & 2.20 & 9 \\
\hline Idaho & $1,251,700$ & 23.84 & 28.08 & 22.43 & 96 & 1.16 & 0.92 & 4 \\
\hline Illinois & $12,128,370$ & 262.92 & 309.64 & 25.53 & 109 & 121.35 & 10.01 & 39 \\
\hline Indiana & $5,942,901$ & 102.11 & 120.25 & 20.23 & 87 & 4.83 & 0.81 & 4 \\
\hline lowa & $2,869,413$ & 53.03 & 62.45 & 21.76 & 93 & 32.87 & 11.46 & 53 \\
\hline Kansas & $2,654,052$ & 45.68 & 53.79 & 20.27 & 87 & 24.82 & 9.35 & 46 \\
\hline Kentucky & $3,960,825$ & 62.77 & 73.93 & 18.66 & 80 & 173.47 & 43.80 & 235 \\
\hline Louisiana & $4,372,035$ & 85.86 & 101.12 & 23.13 & 99 & 300.62 & 68.76 & 297 \\
\hline Maine & $1,253,040$ & 26.98 & 31.77 & 25.35 & 109 & 3.11 & 2.49 & 10 \\
\hline Maryland & $5,171,634$ & 112.20 & 132.13 & 25.55 & 109 & 11.38 & 2.20 & $\frac{x}{9}$ \\
\hline Massachusetts & $6,175,169$ & 133.83 & 157.61 & 25.52 & 109 & 21.69 & 3.51 & 14 \\
\hline Michigan & $9,863,775$ & 187.85 & 221.23 & 22.43 & 96 & 11.99 & 1.22 & 5 \\
\hline Minnesota & $4,775,508$ & 104.18 & 122.69 & 25.69 & 110 & 4.11 & 0.86 & 3 \\
\hline Mississippi & $2,768,619$ & 36.83 & 43.37 & 15.67 & 67 & 71.70 & 25.90 & 165 \\
\hline Missouri & $5,468,338$ & 95.96 & 113.01 & 20.67 & 89 & 102.11 & 18.67 & 90 \\
\hline Montana & 882,779 & 22.55 & 26.56 & 30.08 & 129 & 1.08 & 1.22 & 4 \\
\hline Nebraska & $1,666,028$ & 36.03 & 42.44 & 25.47 & 109 & 5.94 & 3.56 & 14 \\
\hline Nevada & $1,809,253$ & 46.07 & 54.25 & 29.99 & 129 & 19.46 & 10.76 & 36 \\
\hline New Hampshire & $1,201,134$ & 25.80 & 30.39 & 25.30 & 108 & 4.14 & 3.45 & 14 \\
\hline New Jersey & $8,143,412$ & 238.85 & 281.28 & 34.54 & 148 & 149.43 & 18.35 & 53 \\
\hline New Mexico & $1,739,844$ & 25.77 & 30.34 & 17.44 & 75 & 2.29 & 1.31 & 8 \\
\hline New York & $18,196,601$ & 498.42 & 586.98 & 32.26 & 138 & 73.12 & 4.02 & 12 \\
\hline North Carolina & $7,650,789$ & 135.18 & 159.20 & 20.81 & 89 & 227.00 & 29.67 & 143 \\
\hline North Dakota & 633,666 & 11.31 & 13.31 & 21.01 & 90 & 0.00 & 0.00 & 0 \\
\hline Ohio & $11,256,654$ & 185.03 & 217.91 & 19.36 & 83 & 394.45 & 35.04 & 181 \\
\hline Oklahoma & $3,358,044$ & 61.84 & 72.83 & 21.69 & 93 & 38.53 & 11.47 & 53 \\
\hline Oregon & $3,316,154$ & 67.32 & 79.28 & 23.91 & 102 & 5.98 & 1.80 & 8 \\
\hline Pennsylvania & $11,994,016$ & 187.08 & 220.32 & 18.37 & 79 & $1,116.33$ & 93.07 & 507 \\
\hline Rhode Island & $\begin{array}{r}990,819 \\
\end{array}$ & 25.09 & 29.55 & 29.82 & 128 & $\begin{array}{l}12.22 \\
12.02\end{array}$ & 12.34 & 41 \\
\hline South Carolina & $3,885,736$ & 69.59 & 81.96 & 21.09 & 90 & 37.16 & 9.56 & 45 \\
\hline South Dakota & 733,133 & 13.67 & 16.10 & 21.97 & 94 & 1.89 & 2.58 & 12 \\
\hline Tennessee & $5,483,535$ & 63.93 & 75.29 & 13.73 & 59 & 323.38 & 58.97 & 429 \\
\hline Texas & $20,044,141$ & 319.24 & 375.96 & 18.76 & 80 & $2,127.76$ & 106.15 & 566 \\
\hline Utah & $2,129,836$ & 41.62 & 49.02 & 23.02 & 99 & 1.52 & 0.72 & 3 \\
\hline Vermont & 593,740 & 16.03 & 18.87 & 31.79 & 136 & 1.27 & 2.15 & $\frac{5}{7}$ \\
\hline Virginia & $6,872,912$ & 131.34 & 154.67 & 22.50 & 96 & 27.53 & 4.01 & 18 \\
\hline Washington & $5,756,361$ & 107.42 & 126.50 & 21.98 & 94 & 13.17 & 2.29 & 10 \\
\hline West Virginia & $1,806,928$ & 22.25 & 26.20 & 14.50 & 62 & 7.90 & 4.37 & 30 \\
\hline Wisconsin & $5,250,446$ & 92.28 & 108.67 & 20.70 & 89 & 59.92 & 11.41 & 55 \\
\hline Wyoming & 479,602 & 12.00 & 14.13 & 29.45 & 126 & 6.10 & 12.73 & 43 \\
\hline $\begin{array}{l}\text { Totals } \\
\end{array}$ & $\overline{272,690,813}$ & $\overline{5,402.86}$ & $\bar{~} 6,362.83$ & 23.33 & 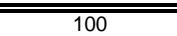 & $\bar{~} 6,362.83$ & 23.33 & $\overline{100}$ \\
\hline
\end{tabular}

RTS rate

$\$ 1177.68 /$ license 
Personal Income Tax

\begin{tabular}{|c|c|c|c|c|c|c|c|c|c|c|c|c|}
\hline State & $\begin{array}{l}1999 \text { State } \\
\text { Population }\end{array}$ & $\begin{array}{l}1999 \text { Adjusted } \\
\text { Gross Income } \\
\text { AGI Amount } \\
\text { (\$ millions) } \\
\end{array}$ & $\begin{array}{l}1999 \text { Total } \\
\text { Exemptions } \\
\text { (\$ millions) }\end{array}$ & $\begin{array}{l}\text { Adjustment for } \\
\text { Residence } \\
\text { (\$ millions) } \\
\end{array}$ & $\begin{array}{l}\text { AGI+Adjustments- } \\
\text { Adjustment for } \\
\text { Residence } \\
\text { (\$ millions) } \\
\end{array}$ & $\begin{array}{c}1999 \text { Base: Individual Income } \\
\text { (AGI-exemptions+l-residency) } \\
\text { (\$ millions) }\end{array}$ & $\begin{array}{l}1999 \text { Tax } \\
\text { Capacity } \\
\text { (\$ millions) }\end{array}$ & $\begin{array}{c}1999 \text { Capacity per } \\
\text { capita } \\
\text { (\$/person) }\end{array}$ & $\begin{array}{l}1999 \text { Per Capita } \\
\text { Capacity Index }\end{array}$ & $\begin{array}{c}1999 \text { Tax } \\
\text { Revenue } \\
\text { (\$ millions) }\end{array}$ & $\begin{array}{l}1999 \text { Revenue } \\
\text { per capita } \\
\text { (\$/person) } \\
\end{array}$ & $\begin{array}{l}1999 \text { Tax } \\
\text { Effort Index }\end{array}$ \\
\hline Alabama & $4,369,862$ & $68,354.9$ & 558.5 & 814.2 & $68,099.2$ & $68,099.2$ & $2,280.7$ & 521.92 & 75 & $2,044.78$ & 467.93 & 90 \\
\hline Alaska & 619,500 & $12,816.3$ & 143.0 & -808.4 & $13,767.7$ & $13,767.7$ & 461.1 & 744.30 & 107 & 0.00 & 0.00 & 0 \\
\hline Arizona & $4,778,332$ & $87,588.7$ & 825.5 & 386.5 & $88,027.7$ & $88,027.7$ & $2,948.1$ & 616.98 & 89 & $2,098.35$ & 439.14 & 71 \\
\hline Arkansas & $2,551,373$ & $36,759.6$ & 329.2 & -279.5 & $37,368.3$ & $37,368.3$ & $1,251.5$ & 490.52 & 71 & $1,433.85$ & 561.99 & 115 \\
\hline California & $33,145,121$ & $707,433.7$ & $8,359.5$ & 151.1 & $715,642.1$ & $715,642.1$ & $23,967.6$ & 723.11 & 104 & $30,732.36$ & 927.21 & 128 \\
\hline Colorado & $4,056,133$ & $94,994.0$ & $1,063.9$ & 110.2 & $95,947.8$ & $95,947.8$ & $3,213.4$ & 792.23 & 114 & $3,354.87$ & 827.11 & 104 \\
\hline Connecticut & $3,282,031$ & $102,922.3$ & $1,108.1$ & $6,107.7$ & $97,922.7$ & $97,922.7$ & $3,279.5$ & 999.24 & 144 & $3,609.99$ & $1,099.93$ & 110 \\
\hline Delaware & 753,538 & $16,778.1$ & 120.7 & $-1,225.1$ & $18,123.9$ & $18,123.9$ & 607.0 & 805.52 & 116 & 813.72 & $1,079.86$ & 134 \\
\hline D.C. & 519,000 & $14,096.2$ & 168.9 & $-26,699.2$ & $14,265.2$ & $14,265.2$ & 477.8 & 920.53 & 133 & 952.16 & $1,834.60$ & 199 \\
\hline Florida & $15,111,244$ & $309,909.2$ & $2,761.2$ & 927.8 & $311,742.6$ & $311,742.6$ & $10,440.6$ & 690.92 & 100 & 0.00 & 0.00 & 0 \\
\hline Georgia & $7,788,240$ & $150,590.1$ & $1,358.0$ & -402.3 & $152,350.4$ & $152,350.4$ & $5,102.4$ & 655.14 & 94 & $5,696.76$ & 731.46 & 112 \\
\hline Hawaii & $1,185,497$ & $21,600.7$ & 220.0 & 0.0 & $21,820.7$ & $21,820.7$ & 730.8 & 616.45 & 89 & $1,068.97$ & 901.71 & 146 \\
\hline Idaho & $1,251,700$ & $19,564.0$ & 225.0 & 347.8 & $19,441.2$ & $19,441.2$ & 651.1 & 520.18 & 75 & 847.02 & 676.70 & 130 \\
\hline Illinois & $12,128,370$ & $274,786.9$ & $2,364.0$ & -796.7 & $277,947.5$ & $277,947.5$ & $9,308.8$ & 767.52 & 111 & $7,247.52$ & 597.57 & 78 \\
\hline Indiana & $5,942,901$ & $112,383.6$ & 904.6 & $2,639.9$ & $110,648.3$ & $110,648.3$ & $3,705.7$ & 623.56 & 90 & $4,214.57$ & 709.18 & 114 \\
\hline lowa & $2,869,413$ & $50,739.8$ & 580.1 & 511.4 & $50,808.5$ & $50,808.5$ & $1,701.6$ & 593.02 & 85 & $1,754.70$ & 611.52 & 103 \\
\hline Kansas & $2,654,052$ & $49,666.0$ & 530.0 & 839.9 & $49,356.1$ & $49,356.1$ & $1,653.0$ & 622.82 & 90 & $1,696.28$ & 639.13 & 103 \\
\hline Kentucky & $3,960,825$ & $61,666.5$ & 546.2 & -746.1 & $62,958.8$ & $62,958.8$ & $2,108.6$ & 532.35 & 77 & $3,221.02$ & 813.22 & 153 \\
\hline Louisiana & $4,372,035$ & $65,130.3$ & 594.5 & -124.2 & $65,849.1$ & $65,849.1$ & $2,205.4$ & 504.42 & 73 & $1,535.65$ & 351.24 & 70 \\
\hline Maine & $1,253,040$ & $21,483.6$ & 273.7 & 283.3 & $21,474.0$ & $21,474.0$ & 719.2 & 573.95 & 83 & $1,020.01$ & 814.03 & 142 \\
\hline Maryland & $5,171,634$ & $123,010.8$ & $1,062.4$ & $17,015.4$ & $124,073.3$ & $124,073.3$ & $4,155.3$ & 803.49 & 116 & $6,563.47$ & $1,269.13$ & 158 \\
\hline Massachusetts & $6,175,169$ & $164,239.7$ & $1,660.6$ & $-3,611.5$ & $169,511.8$ & $169,511.8$ & $5,677.1$ & 919.35 & 132 & $8,036.59$ & $1,301.44$ & 142 \\
\hline Michigan & $9,863,775$ & $201,719.8$ & $1,444.5$ & 849.1 & $202,315.3$ & $202,315.3$ & $6,775.8$ & 686.93 & 99 & $7,475.91$ & 757.92 & 110 \\
\hline Minnesota & $4,775,508$ & $106,979.0$ & $1,075.1$ & -893.3 & $108,947.5$ & $108,947.5$ & $3,648.8$ & 764.06 & 110 & $5,306.24$ & $1,111.14$ & 145 \\
\hline Mississippi & $2,768,619$ & $36,900.1$ & 337.7 & $1,214.3$ & $36,023.4$ & $36,023.4$ & $1,206.5$ & 435.76 & 63 & 983.05 & 355.07 & 81 \\
\hline Missouri & $5,468,338$ & $99,331.2$ & 893.0 & $-3,210.7$ & $103,435.0$ & $103,435.0$ & $3,464.2$ & 633.49 & 91 & $3,855.77$ & 705.11 & 111 \\
\hline Montana & 882,779 & $13,055.0$ & 201.5 & -2.6 & $13,259.0$ & $13,259.0$ & 444.1 & 503.02 & 72 & 483.03 & 547.17 & 109 \\
\hline Nebraska & $1,666,028$ & $31,096.8$ & 346.3 & -602.2 & $32,045.4$ & $32,045.4$ & $1,073.2$ & 644.19 & 93 & $1,071.88$ & 643.38 & 100 \\
\hline Nevada & $1,809,253$ & $42,171.1$ & 348.1 & -745.0 & $43,264.3$ & $43,264.3$ & $1,449.0$ & 800.87 & 115 & 0.00 & 0.00 & 0 \\
\hline New Hampshire & $1,201,134$ & $28,993.5$ & 309.8 & $2,928.9$ & $26,374.5$ & $26,374.5$ & 883.3 & 735.40 & 106 & 63.13 & 52.56 & 7 \\
\hline New Jersey & $8,143,412$ & $222,004.8$ & $1,999.5$ & $17,236.3$ & $206,768.0$ & $206,768.0$ & $6,924.9$ & 850.37 & 122 & $6,353.98$ & 780.26 & 92 \\
\hline New Mexico & $1,739,844$ & $25,833.2$ & 245.6 & 98.3 & $25,980.6$ & $25,980.6$ & 870.1 & 500.11 & 72 & 809.57 & 465.31 & 93 \\
\hline New York & $18,196,601$ & $425,240.4$ & $4,135.7$ & $-24,509.9$ & $453,886.0$ & $453,886.0$ & $15,201.1$ & 835.38 & 120 & $26,045.07$ & $1,431.32$ & 171 \\
\hline North Carolina & $7,650,789$ & $141,801.7$ & $1,259.1$ & -888.5 & $143,949.2$ & $143,949.2$ & $4,821.0$ & 630.13 & 91 & $6,586.15$ & 860.85 & 137 \\
\hline North Dakota & 633,666 & $9,887.7$ & 142.9 & -356.4 & $10,387.0$ & $10,387.0$ & 347.9 & 548.98 & 79 & 181.98 & 287.18 & 52 \\
\hline Ohio & $11,256,654$ & $216,174.6$ & $1,766.2$ & $-1,417.3$ & $219,358.1$ & $219,358.1$ & $7,346.5$ & 652.64 & 94 & $10,288.39$ & 913.98 & 140 \\
\hline Oklahoma & $3,358,044$ & $50,023.8$ & 460.6 & 854.9 & $49,629.4$ & $49,629.4$ & $1,662.1$ & 494.97 & 71 & $2,070.45$ & 616.56 & 125 \\
\hline Oregon & $3,316,154$ & $62,902.2$ & 756.5 & $-1,693.4$ & $65,352.1$ & $65,352.1$ & $2,188.7$ & 660.02 & 95 & $3,709.59$ & $1,118.64$ & 169 \\
\hline Pennsylvania & $11,994,016$ & $240,243.1$ & $2,300.2$ & $1,960.4$ & $240,582.8$ & $240,582.8$ & $8,057.4$ & 671.78 & 97 & $8,846.07$ & 737.54 & 110 \\
\hline Rhode Island & 990,819 & $20,131.7$ & 196.7 & $1,094.4$ & $19,234.0$ & $19,234.0$ & 644.2 & 650.14 & 94 & 762.78 & 769.85 & 118 \\
\hline South Carolina & $3,885,736$ & $64,105.6$ & 545.6 & $1,092.8$ & $63,558.5$ & $63,558.5$ & $2,128.6$ & 547.81 & 79 & $2,298.23$ & 591.45 & 108 \\
\hline South Dakota & 733,133 & $12,060.2$ & 175.0 & -213.1 & $12,448.3$ & $12,448.3$ & 416.9 & 568.67 & 82 & 0.00 & 0.00 & 0 \\
\hline Tennessee & $5,483,535$ & $96,338.3$ & 951.7 & -967.7 & $98,257.8$ & $98,257.8$ & $3,290.8$ & 600.12 & 86 & 160.18 & 29.21 & 5 \\
\hline Texas & $20,044,141$ & $368,614.8$ & $3,182.9$ & -957.6 & $372,755.3$ & $372,755.3$ & $12,484.0$ & 622.82 & 90 & 0.00 & 0.00 & 0 \\
\hline Utah & $2,129,836$ & $36,256.1$ & 294.0 & 23.5 & $36,526.6$ & $36,526.6$ & $1,223.3$ & 574.37 & 83 & $1,461.30$ & 686.11 & 119 \\
\hline Vermont & 593,740 & $11,159.2$ & 155.6 & 103.3 & $11,211.6$ & $\begin{array}{l}11,211.6 \\
\end{array}$ & 375.5 & 632.41 & 91 & 383.45 & 645.82 & 102 \\
\hline Virginia & $6,872,912$ & $152,518.4$ & $1,399.2$ & $7,890.2$ & $153,917.7$ & $153,917.7$ & $5,154.9$ & 750.03 & 108 & $6,087.86$ & 885.78 & 118 \\
\hline Washington & $5,756,361$ & $134,870.5$ & $1,217.0$ & $1,884.1$ & $134,203.5$ & $134,203.5$ & $4,494.6$ & 780.81 & 112 & 0.00 & 0.00 & 0 \\
\hline West Virginia & $1,806,928$ & $24,015.9$ & 224.8 & 398.6 & $23,842.1$ & $23,842.1$ & 798.5 & 441.91 & 64 & 919.95 & 509.12 & 115 \\
\hline Wisconsin & $5,250,446$ & $106,337.9$ & 957.9 & $2,381.6$ & $104,914.2$ & $104,914.2$ & $3,513.7$ & 669.22 & 96 & $5,162.24$ & 983.20 & 147 \\
\hline Wyoming & 479,602 & $9,673.7$ & 95.6 & -21.1 & $9,790.5$ & $9,790.5$ & 327.9 & 683.68 & 98 & 0.00 & 0.00 & 0 \\
\hline $\begin{array}{l}\text { Totals } \\
\end{array}$ & $272,690,813$ & $5,597,681.7$ & 53,806.4 & $-1,026.0$ & $5,652,514.1$ & $5,652,514.1$ & $\begin{array}{l}189,308.9 \\
\end{array}$ & $\begin{array}{c}694.23 \\
\end{array}$ & 100 & $189,308.85$ & 6994.23 & 100 \\
\hline
\end{tabular}


Corporate Income Tax

\begin{tabular}{|c|c|c|c|c|c|c|c|c|}
\hline State & $\begin{array}{l}1999 \text { State } \\
\text { Population }\end{array}$ & $\begin{array}{c}1999 \text { Total Corporate Income Tax } \\
\text { Base (equal to allocated profits) } \\
\text { (\$ millions) }\end{array}$ & $\begin{array}{l}1999 \text { Tax Capacity } \\
\text { (\$ millions) }\end{array}$ & $\begin{array}{l}1999 \text { Capacity per } \\
\text { capita } \\
\text { (\$/person) } \\
\end{array}$ & $\begin{array}{l}1999 \text { Per Capita Capacity } \\
\text { Index }\end{array}$ & $\begin{array}{c}1999 \text { Tax } \\
\text { Revenue } \\
\text { (\$ millions) } \\
\end{array}$ & $\begin{array}{c}1999 \text { Revenue per } \\
\text { capita } \\
\text { (\$/person) } \\
\end{array}$ & $\begin{array}{l}1999 \text { Tax Effort } \\
\text { Index }\end{array}$ \\
\hline Alabama & $4,369,862$ & $7,755.0$ & 417.3 & 95.50 & 77 & 233.02 & 53.33 & 56 \\
\hline Alaska & 619,500 & $1,072.0$ & 57.7 & 93.12 & 75 & 211.81 & 341.91 & 367 \\
\hline Arizona & $4,778,332$ & $9,319.4$ & 501.5 & 104.96 & 84 & 545.39 & 114.14 & 109 \\
\hline Arkansas & $2,551,373$ & $4,595.3$ & 247.3 & 96.92 & 78 & 212.21 & 83.17 & 86 \\
\hline California & $33,145,121$ & $71,259.8$ & $3,834.8$ & 115.70 & 93 & $5,459.20$ & 164.71 & 142 \\
\hline Colorado & $4,056,133$ & $10,673.5$ & 574.4 & 141.61 & 114 & 301.04 & 74.22 & 52 \\
\hline Connecticut & $3,282,031$ & $10,527.6$ & 566.5 & 172.62 & 139 & 474.51 & 144.58 & 84 \\
\hline Delaware & 753,538 & $4,112.3$ & 221.3 & 293.68 & 236 & 232.53 & 308.58 & 105 \\
\hline D.C. & 519,000 & $3,779.9$ & 203.4 & 391.93 & 315 & 217.60 & 419.26 & 107 \\
\hline Florida & $15,111,244$ & $27,540.4$ & $1,482.1$ & 98.08 & 79 & $1,266.96$ & 83.84 & 85 \\
\hline Georgia & $7,788,240$ & $19,190.1$ & $1,032.7$ & 132.60 & 107 & 793.17 & 101.84 & 77 \\
\hline Hawaii & $1,185,497$ & $1,995.3$ & 107.4 & 90.57 & 73 & 52.41 & 44.21 & 49 \\
\hline Idaho & $1,251,700$ & $1,974.1$ & 106.2 & 84.87 & 68 & 96.15 & 76.81 & 91 \\
\hline Illinois & $12,128,370$ & $33,754.8$ & $1,816.5$ & 149.77 & 120 & $2,103.93$ & 173.47 & 116 \\
\hline Indiana & $5,942,901$ & $13,670.1$ & 735.6 & 123.79 & 100 & 989.63 & 166.52 & 135 \\
\hline $\begin{array}{l}\text { lowa } \\
\end{array}$ & $2,869,413$ & $6,460.0$ & 347.6 & 121.15 & 97 & 234.54 & 81.74 & 67 \\
\hline Kansas & $2,654,052$ & $5,747.0$ & 309.3 & 116.53 & 94 & 253.84 & 95.64 & 82 \\
\hline Kentucky & $3,960,825$ & $7,677.3$ & 413.1 & 104.31 & 84 & 312.07 & 78.79 & 76 \\
\hline Louisiana & $4,372,035$ & $7,681.6$ & 413.4 & 94.55 & 76 & 286.32 & 65.49 & 69 \\
\hline Maine & $1,253,040$ & $2,149.7$ & 115.7 & 92.32 & 74 & 147.49 & 117.70 & 127 \\
\hline Maryland & $5,171,634$ & $9,929.7$ & 534.4 & 103.33 & 83 & 404.97 & 78.31 & 76 \\
\hline Massachusetts & $6,175,169$ & $18,061.0$ & 971.9 & 157.40 & 127 & $1,249.67$ & 202.37 & 129 \\
\hline Michigan & $9,863,775$ & $23,564.7$ & $1,268.1$ & 128.56 & 103 & $2,413.62$ & 244.69 & 190 \\
\hline Minnesota & $4,775,508$ & $12,931.9$ & 695.9 & 145.73 & 117 & 779.18 & 163.16 & 112 \\
\hline Mississippi & $2,768,619$ & $4,119.8$ & 221.7 & 80.08 & 64 & 229.50 & 82.89 & 104 \\
\hline Missouri & $5,468,338$ & $12,684.5$ & 682.6 & 124.83 & 100 & 276.52 & 50.57 & 41 \\
\hline Montana & 882,779 & $1,174.6$ & 63.2 & 71.60 & 58 & 89.62 & 101.52 & 142 \\
\hline Nebraska & $1,666,028$ & $3,708.2$ & 199.6 & 119.78 & 96 & 135.03 & 81.05 & 68 \\
\hline Nevada & $1,809,253$ & $3,994.7$ & 215.0 & 118.82 & 96 & 0.00 & 0.00 & 0 \\
\hline New Hampshire & $1,201,134$ & $2,473.6$ & 133.1 & 110.82 & 89 & 255.82 & 212.98 & 192 \\
\hline New Jersey & $8,143,412$ & $22,274.0$ & $1,198.7$ & 147.19 & 118 & $1,333.96$ & 163.81 & 111 \\
\hline New Mexico & $1,739,844$ & $2,170.2$ & 116.8 & 67.13 & 54 & 163.96 & 94.24 & 140 \\
\hline New York & $18,196,601$ & $59,226.6$ & $3,187.2$ & 175.16 & 141 & $5,827.19$ & 320.24 & 183 \\
\hline North Carolina & $7,650,789$ & $18,589.2$ & $1,000.4$ & 130.75 & 105 & 920.58 & 120.33 & 92 \\
\hline North Dakota & 633,666 & $1,116.2$ & 60.1 & 94.80 & 76 & 93.60 & 147.70 & 156 \\
\hline Ohio & $11,256,654$ & $26,608.9$ & $1,431.9$ & 127.21 & 102 & 751.57 & 66.77 & 52 \\
\hline Oklahoma & $3,358,044$ & $5,184.1$ & 279.0 & 83.08 & 67 & 187.31 & 55.78 & 67 \\
\hline Oregon & $3,316,154$ & $6,716.0$ & 361.4 & 108.99 & 88 & 324.39 & 97.82 & 90 \\
\hline Pennsylvania & $11,994,016$ & $27,909.0$ & $1,501.9$ & 125.22 & 101 & $1,537.72$ & 128.21 & 102 \\
\hline Rhode Island & 990,819 & $2,062.3$ & 111.0 & 112.01 & 90 & 66.32 & 66.93 & 60 \\
\hline South Carolina & $3,885,736$ & $7,442.7$ & 400.5 & 103.07 & 83 & 257.49 & 66.26 & 64 \\
\hline South Dakota & 733,133 & $1,677.8$ & 90.3 & 123.15 & 99 & 50.82 & 69.32 & 56 \\
\hline Tennessee & $5,483,535$ & $11,751.8$ & 632.4 & 115.33 & 93 & 571.43 & 104.21 & 90 \\
\hline Texas & $20,044,141$ & $46,628.2$ & $2,509.3$ & 125.19 & 101 & 0.00 & 0.00 & 0 \\
\hline Utah & $2,129,836$ & $4,330.9$ & 233.1 & 109.43 & 88 & 180.14 & 84.58 & 77 \\
\hline Vermont & 593,740 & $1,059.3$ & 57.0 & 96.01 & 77 & 49.70 & 83.70 & 87 \\
\hline Virginia & $6,872,912$ & $16,156.9$ & 869.5 & 126.51 & 102 & 414.39 & 60.29 & 48 \\
\hline Washington & $5,756,361$ & $12,586.4$ & 677.3 & 117.67 & 95 & 0.00 & 0.00 & 0 \\
\hline West Virginia & $1,806,928$ & $2,613.4$ & 140.6 & 77.83 & 63 & 263.12 & 145.62 & 187 \\
\hline Wisconsin & $5,250,446$ & $12,021.9$ & 646.9 & 123.22 & 99 & 670.96 & 127.79 & 104 \\
\hline Wyoming & 479,602 & 742.5 & 40.0 & 83.31 & 67 & 0.00 & 0.00 & 0 \\
\hline Totals & $\begin{array}{l}272,690,813 \\
\end{array}$ & (630,359.0 & $\begin{array}{l}33,922.4 \\
\end{array}$ & $\begin{array}{l}124.40 \\
\end{array}$ & 100 & $\begin{array}{l}33,922.37 \\
\end{array}$ & $\begin{array}{l}124.40 \\
\end{array}$ & 100 \\
\hline
\end{tabular}


Property Taxes--Total

\begin{tabular}{|c|c|c|c|c|c|c|c|c|c|c|c|c|}
\hline State & $\begin{array}{l}1999 \text { State } \\
\text { Population }\end{array}$ & $\begin{array}{c}1999 \text { Residential } \\
\text { Property Tax Base } \\
\text { (\$ millions) }\end{array}$ & $\begin{array}{l}1999 \text { Utility } \\
\text { Property Tax } \\
\text { Base } \\
\text { (\$ millions) } \\
\end{array}$ & $\begin{array}{c}1999 \text { Corporate } \\
\text { Property Tax Base } \\
(\$ \text { millions })\end{array}$ & $\begin{array}{l}1999 \text { Farm } \\
\text { Property Tax } \\
\text { Base } \\
\text { (\$ millions) }\end{array}$ & $\begin{array}{l}1999 \text { Total } \\
\text { Property Tax } \\
\text { Base } \\
\text { (\$ millions) } \\
\end{array}$ & $\begin{array}{l}1999 \text { Tax } \\
\text { Capacity } \\
\text { (\$ millions) }\end{array}$ & $\begin{array}{c}1999 \text { Capacity per } \\
\text { capita } \\
\text { (\$/person) }\end{array}$ & $\begin{array}{l}1999 \text { Per Capita } \\
\text { Capacity Index }\end{array}$ & $\begin{array}{l}1999 \text { Tax Revenue } \\
\text { (\$ millions) }\end{array}$ & $\begin{array}{l}1999 \text { Revenue } \\
\text { per capita } \\
\text { (\$/person) } \\
\end{array}$ & $\begin{array}{l}1999 \text { Tax Effort } \\
\text { Index }\end{array}$ \\
\hline$\overline{\text { Alabama }}$ & $4,369,862$ & $140,704.8$ & $4,587.1$ & $87,340.1$ & $13,984.0$ & $246,616.00$ & $2,927.77$ & 669.99 & 76 & $1,191.79$ & 272.73 & 41 \\
\hline Alaska & 619,500 & $24,579.7$ & 5.8 & $26,607.1$ & 301.2 & $51,493.77$ & 611.32 & 986.80 & 112 & 727.81 & $1,174.84$ & 119 \\
\hline Arizona & $4,778,332$ & $207,811.8$ & $5,831.9$ & $123,988.7$ & $29,425.0$ & $367,057.37$ & $4,357.62$ & 911.95 & 104 & $3,584.16$ & 750.08 & 82 \\
\hline Arkansas & $2,551,373$ & $72,065.5$ & $2,304.4$ & $55,458.8$ & $17,873.0$ & $147,701.66$ & $1,753.48$ & 687.27 & 78 & 966.75 & 378.91 & 55 \\
\hline California & $33,145,121$ & $2,044,321.5$ & $46,791.8$ & $1,141,741.6$ & $79,230.0$ & $3,312,084.96$ & $39,320.31$ & $1,186.31$ & 135 & $25,424.96$ & 767.08 & 65 \\
\hline Colorado & $4,056,133$ & $155,668.6$ & $3,614.1$ & $75,249.5$ & $12,656.9$ & $247,189.13$ & $2,934.57$ & 723.49 & 82 & $3,413.61$ & 841.59 & 116 \\
\hline Connecticut & $3,282,031$ & $214,306.2$ & $4,881.9$ & $106,426.7$ & $2,331.0$ & $327,945.91$ & $3,893.30$ & $1,186.25$ & 135 & $5,174.84$ & $1,576.72$ & 133 \\
\hline Delaware & 753,538 & $15,001.6$ & 513.1 & $50,778.8$ & 692.5 & $66,986.00$ & 795.24 & $1,055.34$ & 120 & 348.52 & 462.51 & 44 \\
\hline D.C. & 519,000 & $31,024.3$ & $2,096.8$ & $13,177.4$ & 0.0 & $46,298.48$ & 549.64 & $1,059.05$ & 120 & 679.55 & $1,309.34$ & 124 \\
\hline Florida & $15,111,244$ & $650,837.0$ & $22,368.4$ & $381,276.1$ & $23,504.0$ & $1,077,985.41$ & $12,797.60$ & 846.89 & 96 & $13,900.95$ & 919.91 & 109 \\
\hline Georgia & $7,788,240$ & $313,082.5$ & $9,104.0$ & $187,465.0$ & $18,256.0$ & $527,907.54$ & $6,267.20$ & 804.70 & 92 & $5,422.82$ & 696.28 & 87 \\
\hline Hawaii & $1,185,497$ & $98,282.9$ & $1,953.8$ & $28,309.7$ & $4,989.9$ & $133,536.22$ & $1,585.31$ & $1,337.25$ & 152 & 594.56 & 501.53 & 38 \\
\hline Idaho & $1,251,700$ & $33,296.6$ & 986.1 & $26,309.1$ & $8,963.1$ & $69,554.93$ & 825.74 & 659.69 & 75 & 815.66 & 651.64 & 99 \\
\hline Illinois & $12,128,370$ & $562,965.8$ & $16,069.8$ & $305,198.1$ & $62,325.0$ & $946,558.66$ & $11,237.33$ & 926.53 & 105 & $14,099.97$ & $1,162.56$ & 125 \\
\hline Indiana & $5,942,901$ & $212,309.0$ & $7,153.0$ & $132,384.0$ & $34,410.0$ & $386,256.00$ & $4,585.54$ & 771.60 & 88 & $5,177.13$ & 871.15 & 113 \\
\hline lowa & $2,869,413$ & $93,064.7$ & $2,917.8$ & $68,195.5$ & $58,410.0$ & $222,588.00$ & $2,642.51$ & 920.92 & 105 & $2,532.74$ & 882.67 & 96 \\
\hline Kansas & $2,654,052$ & $84,146.0$ & $3,009.8$ & $64,022.5$ & $27,550.0$ & $178,728.26$ & $2,121.82$ & 799.46 & 91 & $2,115.02$ & 796.90 & 100 \\
\hline Kentucky & $3,960,825$ & $124,151.4$ & $4,094.5$ & $85,306.8$ & $20,808.0$ & $234,360.72$ & $2,782.28$ & 702.45 & 80 & $1,666.33$ & 420.70 & 60 \\
\hline Louisiana & $4,372,035$ & $126,625.4$ & $4,841.9$ & $85,524.6$ & $9,861.5$ & $226,853.33$ & $2,693.15$ & 615.99 & 70 & $1,620.13$ & 370.57 & 60 \\
\hline Maine & $1,253,040$ & $48,700.8$ & $1,473.0$ & $39,288.5$ & $1,524.0$ & $90,986.31$ & $1,080.17$ & 862.04 & 98 & $1,546.86$ & $1,234.48$ & 143 \\
\hline Maryland & $5,171,634$ & $268,867.0$ & $7,944.3$ & $110,508.1$ & $6,930.0$ & $394,249.30$ & $4,680.44$ & 905.02 & 103 & $4,144.06$ & 801.31 & 89 \\
\hline Massachusetts & $6,175,169$ & $333,470.9$ & $7,958.9$ & $153,213.1$ & $2,658.5$ & $497,301.35$ & $5,903.85$ & 956.06 & 109 & $7,300.56$ & $1,182.24$ & 124 \\
\hline Michigan & $9,863,775$ & $420,532.7$ & $12,589.3$ & $247,236.4$ & $19,240.0$ & $699,598.42$ & $8,305.47$ & 842.02 & 96 & $8,810.59$ & 893.23 & 106 \\
\hline Minnesota & $4,775,508$ & $215,400.8$ & $4,782.9$ & $126,389.2$ & $35,424.0$ & $381,996.90$ & $4,534.98$ & 949.63 & 108 & $4,458.85$ & 933.69 & 98 \\
\hline Mississippi & $2,768,619$ & $72,076.1$ & $2,667.2$ & $54,088.2$ & $12,540.0$ & $141,371.49$ & $1,678.33$ & 606.20 & 69 & $1,389.92$ & 502.03 & 83 \\
\hline Missouri & $5,468,338$ & $193,542.6$ & $6,853.4$ & $114,965.0$ & $34,013.0$ & $349,374.01$ & $4,147.69$ & 758.49 & 86 & $3,305.36$ & 604.45 & 80 \\
\hline Montana & 882,779 & $34,302.9$ & 788.2 & $19,326.8$ & $16,872.0$ & $71,289.86$ & 846.34 & 958.72 & 109 & 891.13 & $1,009.46$ & 105 \\
\hline Nebraska & $1,666,028$ & $53,962.6$ & $1,786.5$ & $39,673.1$ & $31,088.0$ & $126,510.26$ & $1,501.90$ & 901.49 & 103 & $1,567.01$ & 940.57 & 104 \\
\hline Nevada & $1,809,253$ & $86,428.5$ & $2,596.1$ & $44,494.0$ & $2,856.0$ & $136,374.49$ & $1,619.01$ & 894.85 & 102 & $1,261.14$ & 697.05 & 78 \\
\hline New Hampshire & $1,201,134$ & $55,915.5$ & $1,669.9$ & $32,006.4$ & 945.0 & $90,536.86$ & $1,074.83$ & 894.85 & 102 & $2,014.40$ & $1,677.08$ & 187 \\
\hline New Jersey & $8,143,412$ & $477,158.6$ & $14,110.0$ & $207,139.1$ & $5,810.0$ & $704,217.75$ & $8,360.31$ & $1,026.63$ & 117 & $14,336.03$ & $1,760.44$ & 171 \\
\hline New Mexico & $1,739,844$ & $64,589.9$ & $1,918.3$ & $29,287.3$ & $9,699.9$ & $105,495.42$ & $1,252.42$ & 719.84 & 82 & 587.85 & 337.87 & 47 \\
\hline New York & $18,196,601$ & $896,092.3$ & $26,026.8$ & $462,505.1$ & $10,452.0$ & $1,395,076.19$ & $16,562.02$ & 910.17 & 104 & $24,758.69$ & $1,360.62$ & 149 \\
\hline North Carolina & $7,650,789$ & $305,715.6$ & $9,206.9$ & $186,216.8$ & $20,925.0$ & $522,064.36$ & $6,197.83$ & 810.09 & 92 & $4,350.64$ & 568.65 & 70 \\
\hline North Dakota & 633,666 & $15,690.4$ & 540.2 & $14,018.8$ & $15,996.4$ & $46,245.77$ & 549.02 & 866.42 & 99 & 497.22 & 784.67 & 91 \\
\hline Ohio & $11,256,654$ & $429,863.7$ & $13,505.1$ & $246,959.8$ & $33,078.0$ & $723,406.62$ & $8,588.12$ & 762.94 & 87 & $9,334.35$ & 829.23 & 109 \\
\hline Oklahoma & $3,358,044$ & $90,969.2$ & $3,715.2$ & $61,007.1$ & $21,250.0$ & $176,941.43$ & $2,100.61$ & 625.55 & 71 & $1,237.65$ & 368.56 & 59 \\
\hline Oregon & $3,316,154$ & $169,586.3$ & $4,084.9$ & $121,143.0$ & $17,200.0$ & $312,014.18$ & $3,704.16$ & $1,117.01$ & 127 & $2,558.19$ & 771.43 & 69 \\
\hline Pennsylvania & $11,994,016$ & $458,634.6$ & $16,626.3$ & $267,005.3$ & $19,250.0$ & $761,516.19$ & $9,040.55$ & 753.75 & 86 & $9,659.06$ & 805.32 & 107 \\
\hline Rhode Island & 990,819 & $45,192.6$ & $1,377.4$ & $22,471.9$ & 390.0 & $69,431.94$ & 824.28 & 831.92 & 95 & $1,285.11$ & $1,297.02$ & 156 \\
\hline South Carolina & $3,885,736$ & $138,146.2$ & $3,467.9$ & $78,236.5$ & $7,372.0$ & $227,222.54$ & $2,697.53$ & 694.21 & 79 & $2,475.95$ & 637.19 & 92 \\
\hline South Dakota & 733,133 & $20,335.7$ & 594.5 & $16,801.5$ & $15,840.0$ & $53,571.74$ & 635.99 & 867.50 & 99 & 617.29 & 841.99 & 97 \\
\hline Tennessee & $5,483,535$ & $202,616.6$ & $5,982.9$ & $116,173.6$ & $23,205.0$ & $347,978.10$ & $4,131.12$ & 753.37 & 86 & $2,684.03$ & 489.47 & 65 \\
\hline Texas & $20,044,141$ & $601,468.8$ & $24,678.7$ & $508,468.7$ & $79,605.0$ & $1,214,221.24$ & $14,414.96$ & 719.16 & 82 & $18,804.96$ & 938.18 & 130 \\
\hline Utah & $2,129,836$ & $93,721.8$ & $2,381.4$ & $35,575.1$ & $9,918.0$ & $141,596.28$ & $1,681.00$ & 789.26 & 90 & $1,191.69$ & 559.52 & 71 \\
\hline Vermont & 593,740 & $25,674.1$ & 719.4 & $15,075.4$ & $2,103.8$ & $43,572.72$ & 517.29 & 871.23 & 99 & 765.69 & $1,289.60$ & 148 \\
\hline Virginia & $6,872,912$ & $322,171.1$ & $9,770.0$ & $149,567.9$ & $17,544.0$ & $499,053.07$ & $5,924.64$ & 862.03 & 98 & $5,757.55$ & 837.72 & 97 \\
\hline Washington & $5,756,361$ & $326,582.2$ & $7,306.9$ & $206,911.7$ & $18,683.0$ & $559,483.82$ & $6,642.06$ & $1,153.86$ & 131 & $5,763.41$ & $1,001.22$ & 87 \\
\hline West Virginia & $1,806,928$ & $49,050.3$ & $1,794.2$ & $25,092.0$ & $3,852.0$ & $79,788.52$ & 947.23 & 524.22 & 60 & 811.77 & 449.25 & 86 \\
\hline Wisconsin & $5,250,446$ & $210,952.0$ & $5,497.4$ & $130,288.2$ & $22,331.0$ & $369,068.65$ & $4,381.50$ & 834.50 & 95 & $5,524.61$ & $1,052.22$ & 126 \\
\hline Wyoming & 479,602 & $18,549.8$ & 539.7 & $12,403.2$ & $7,612.0$ & $39,104.73$ & 464.24 & 967.97 & 110 & 522.70 & $1,089.86$ & 113 \\
\hline $\begin{array}{l}\text { Totals } \\
\end{array}$ & $\overline{\overline{272,690,813}}$ & 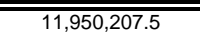 & (348,079.6 & 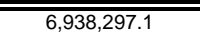 & 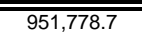 & 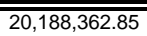 & 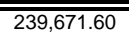 & (878.91 & 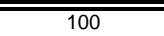 & 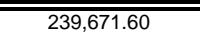 & "878.91 & 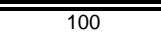 \\
\hline
\end{tabular}


Estate and Gift Taxes

1999 State and Local

\begin{tabular}{|c|c|c|c|c|c|c|c|c|c|c|}
\hline State & 1999 State Population & $\begin{array}{l}1999 \text { Total Base (total } \\
\text { Federal collections) } \\
\text { (\$ millions) }\end{array}$ & $\begin{array}{l}\text { Estate and Gift Tax } \\
\text { Collections } \\
\text { (\$ millions) }\end{array}$ & $\begin{array}{l}1999 \text { Tax } \\
\text { Capacity } \\
\text { (\$ millions) }\end{array}$ & $\begin{array}{l}1999 \text { Capacity } \\
\text { per capita } \\
\text { (\$/person) }\end{array}$ & $\begin{array}{l}1999 \text { Per Capita } \\
\text { Capacity Index }\end{array}$ & $\begin{array}{l}1999 \text { Tax Revenue } \\
\text { (\$ millions) }\end{array}$ & $\begin{array}{c}1999 \text { Revenue per } \\
\text { capita } \\
\text { (\$/person) }\end{array}$ & $\begin{array}{l}1999 \text { Tax Effort } \\
\text { Index }\end{array}$ & $\begin{array}{l}\text { Revenue divided } \\
\text { by base }\end{array}$ \\
\hline Alabama & $4,369,862$ & 281.67 & 62.78 & 74.62 & 17.07 & 62 & 62.78 & 14.37 & 84 & $22.3 \%$ \\
\hline Alaska & 619,500 & 14.92 & 1.73 & 3.95 & 6.38 & 23 & 1.73 & 2.79 & 44 & $11.6 \%$ \\
\hline Arizona & $4,778,332$ & 435.38 & 89.09 & 115.33 & 24.14 & 88 & 89.09 & 18.64 & 77 & $20.5 \%$ \\
\hline Arkansas & $2,551,373$ & 164.12 & 32.57 & 43.48 & 17.04 & 62 & 32.57 & 12.77 & 75 & $19.8 \%$ \\
\hline California & $33,145,121$ & $3,829.89$ & 877.90 & $1,014.54$ & 30.61 & 111 & 877.90 & 26.49 & 87 & $22.9 \%$ \\
\hline Colorado & $4,056,133$ & 327.79 & 65.39 & 86.83 & 21.41 & 78 & 65.39 & 16.12 & 75 & $19.9 \%$ \\
\hline Connecticut & $3,282,031$ & 597.77 & 250.17 & 158.35 & 48.25 & 175 & 250.17 & 76.22 & 158 & $41.9 \%$ \\
\hline Delaware & 753,538 & 116.47 & 27.06 & 30.85 & 40.95 & 148 & 27.06 & 35.91 & 88 & $23.2 \%$ \\
\hline D.C. & 519,000 & 83.21 & 26.25 & 22.04 & 42.47 & 154 & 26.25 & 50.57 & 119 & $31.5 \%$ \\
\hline Florida & $15,111,244$ & $2,454.87$ & 649.52 & 650.30 & 43.03 & 156 & 649.52 & 42.98 & 100 & $26.5 \%$ \\
\hline Georgia & $7,788,240$ & 589.81 & 111.19 & 156.24 & 20.06 & 73 & 111.19 & 14.28 & 71 & $18.9 \%$ \\
\hline Hawaii & $1,185,497$ & 109.58 & 28.74 & 29.03 & 24.49 & 89 & 28.74 & 24.24 & 99 & $26.2 \%$ \\
\hline Idaho & $1,251,700$ & 50.11 & 11.13 & 13.27 & 10.60 & 38 & 11.13 & 8.89 & 84 & $22.2 \%$ \\
\hline Illinois & $12,128,370$ & $1,505.42$ & 346.98 & 398.79 & 32.88 & 119 & 346.98 & 28.61 & 87 & $23.0 \%$ \\
\hline Indiana & $5,942,901$ & 396.82 & 148.71 & 105.12 & 17.69 & 64 & 148.71 & 25.02 & 141 & $37.5 \%$ \\
\hline lowa & $2,869,413$ & 144.15 & 72.84 & 38.19 & 13.31 & 48 & 72.84 & 25.38 & 191 & $50.5 \%$ \\
\hline Kansas & $2,654,052$ & 235.73 & 70.24 & 62.45 & 23.53 & 85 & 70.24 & 26.46 & 112 & $29.8 \%$ \\
\hline Kentucky & $3,960,825$ & 213.70 & 81.48 & 56.61 & 14.29 & 52 & 81.48 & 20.57 & 144 & $38.1 \%$ \\
\hline Louisiana & $4,372,035$ & 335.00 & 95.97 & 88.74 & 20.30 & 74 & 95.97 & 21.95 & 108 & $28.6 \%$ \\
\hline Maine & $1,253,040$ & 89.90 & 29.77 & 23.81 & 19.00 & 69 & 29.77 & 23.76 & 125 & $33.1 \%$ \\
\hline Maryland & $5,171,634$ & 678.82 & 126.17 & 179.82 & 34.77 & 126 & 126.17 & 24.40 & 70 & $18.6 \%$ \\
\hline Massachusetts & $6,175,169$ & 901.71 & 173.87 & 238.86 & 38.68 & 140 & 173.87 & 28.16 & 73 & $19.3 \%$ \\
\hline Michigan & $9,863,775$ & 789.47 & 174.89 & 209.13 & 21.20 & 77 & 174.89 & 17.73 & 84 & $22.2 \%$ \\
\hline Minnesota & $4,775,508$ & 319.80 & 58.13 & 84.72 & 17.74 & 64 & 58.13 & 12.17 & 69 & $18.2 \%$ \\
\hline Mississippi & $2,768,619$ & 142.61 & 30.77 & 37.78 & 13.64 & 49 & 30.77 & 11.11 & 81 & $21.6 \%$ \\
\hline Missouri & $5,468,338$ & 519.22 & 118.67 & 137.54 & 25.15 & 91 & 118.67 & 21.70 & 86 & $22.9 \%$ \\
\hline Montana & 882,779 & 40.41 & 18.30 & 10.71 & 12.13 & 44 & 18.30 & 20.73 & 171 & $45.3 \%$ \\
\hline Nebraska & $1,666,028$ & 125.89 & 17.45 & 33.35 & 20.02 & 73 & 17.45 & 10.47 & 52 & $13.9 \%$ \\
\hline Nevada & $1,809,253$ & 245.61 & 41.47 & 65.06 & 35.96 & 130 & 41.47 & 22.92 & 64 & $16.9 \%$ \\
\hline New Hampshire & $1,201,134$ & 99.64 & 49.37 & 26.39 & 21.97 & 80 & 49.37 & 41.10 & 187 & $49.5 \%$ \\
\hline New Jersey & $8,143,412$ & 938.27 & 423.02 & 248.55 & 30.52 & 111 & 423.02 & 51.95 & 170 & $45.1 \%$ \\
\hline New Mexico & $1,739,844$ & 119.44 & 21.91 & 31.64 & 18.19 & 66 & 21.91 & 12.59 & 69 & $18.3 \%$ \\
\hline New York & $18,196,601$ & $2,989.32$ & $1,071.46$ & 791.87 & 43.52 & 158 & $1,071.46$ & 58.88 & 135 & $35.8 \%$ \\
\hline North Carolina & $7,650,789$ & 633.47 & 182.85 & 167.81 & 21.93 & 80 & 182.85 & 23.90 & 109 & $28.9 \%$ \\
\hline North Dakota & 633,666 & 22.14 & 7.42 & 5.87 & 9.26 & 34 & 7.42 & 11.70 & 126 & $33.5 \%$ \\
\hline Ohio & $11,256,654$ & $1,469.89$ & 141.46 & 389.38 & 34.59 & 125 & 141.46 & 12.57 & 36 & $9.6 \%$ \\
\hline Oklahoma & $3,358,044$ & 259.17 & 88.80 & 68.65 & 20.44 & 74 & 88.80 & 26.44 & 129 & $34.3 \%$ \\
\hline Oregon & $3,316,154$ & 222.33 & 47.98 & 58.90 & 17.76 & 64 & 47.98 & 14.47 & 81 & $21.6 \%$ \\
\hline Pennsylvania & $11,994,016$ & $1,232.29$ & 760.70 & 326.43 & 27.22 & 99 & 760.70 & 63.42 & 233 & $61.7 \%$ \\
\hline Rhode Island & $\begin{array}{l}990,819 \\
\end{array}$ & 121.56 & 46.85 & 32.20 & 32.50 & 118 & 46.85 & 47.29 & 146 & $38.5 \%$ \\
\hline South Carolina & $3,885,736$ & 272.41 & 57.19 & 72.16 & 18.57 & 67 & 57.19 & 14.72 & 79 & $21.0 \%$ \\
\hline South Dakota & 733,133 & 29.91 & 26.43 & 7.92 & 10.81 & 39 & 26.43 & 36.05 & 334 & $88.4 \%$ \\
\hline Tennessee & $5,483,535$ & 348.83 & 89.13 & 92.40 & 16.85 & 61 & 89.13 & 16.25 & 96 & $25.6 \%$ \\
\hline Texas & $20,044,141$ & $1,414.90$ & 256.28 & 374.81 & 18.70 & 68 & 256.28 & 12.79 & 68 & $18.1 \%$ \\
\hline Utah & $2,129,836$ & 105.12 & 8.24 & 27.85 & 13.07 & 47 & 8.24 & 3.87 & 30 & $7.8 \%$ \\
\hline Vermont & 593,740 & 94.46 & 23.36 & 25.02 & 42.14 & 153 & 23.36 & 39.34 & 93 & $24.7 \%$ \\
\hline Virginia & $6,872,912$ & 762.56 & 154.08 & 202.00 & 29.39 & 107 & 154.08 & 22.42 & 76 & $20.2 \%$ \\
\hline Washington & $5,756,361$ & 370.63 & 69.70 & 98.18 & 17.06 & 62 & 69.70 & 12.11 & 71 & $18.8 \%$ \\
\hline West Virginia & $1,806,928$ & 87.64 & 27.33 & 23.22 & 12.85 & 47 & 27.33 & 15.12 & 118 & $31.2 \%$ \\
\hline Wisconsin & $5,250,446$ & 393.40 & 116.90 & 104.21 & 19.85 & 72 & 116.90 & 22.26 & 112 & $29.7 \%$ \\
\hline Wyoming & 479,602 & 65.78 & 9.73 & 17.43 & 36.33 & 132 & 9.73 & 20.29 & 56 & $14.8 \%$ \\
\hline Totals & $\overline{272,690,813}$ & $28,385.61$ & $7,519.38$ & $7,519.38$ & & & $7,7,519.38$ & & & $26.5 \%$ \\
\hline
\end{tabular}

RTS rate

26.49 cents per dollar 
Severance Taxes

\begin{tabular}{|c|c|c|c|c|c|c|c|c|c|c|c|c|}
\hline State & $\begin{array}{l}1999 \text { State } \\
\text { Population }\end{array}$ & $\begin{array}{l}\begin{array}{l}\text { Value of Oil } \\
\text { (\$ millions) }\end{array} \\
\end{array}$ & $\begin{array}{c}\text { Value of Coal } \\
\text { (\$ millions) }\end{array}$ & $\begin{array}{l}\text { Value of Gas } \\
\text { (\$ millions) }\end{array}$ & $\begin{array}{c}\text { Value of non-fuel } \\
\text { mineral } \\
(\$ \text { millions }) \\
\end{array}$ & $\begin{array}{c}1999 \text { Total Severance Tax } \\
\text { Base (sum of values) } \\
\text { (\$ millions) }\end{array}$ & $\begin{array}{l}1999 \text { Tax } \\
\text { Capacity } \\
\text { (\$ millions) }\end{array}$ & $\begin{array}{l}1999 \text { Capacity } \\
\text { per capita } \\
\text { (\$/person) } \\
\end{array}$ & $\begin{array}{l}1999 \text { Per Capita } \\
\text { Capacity Index }\end{array}$ & $\begin{array}{c}1999 \text { Tax } \\
\text { Revenue } \\
\text { (\$ millions) } \\
\end{array}$ & $\begin{array}{c}1999 \text { Revenue per } \\
\text { capita } \\
\text { (\$/person) }\end{array}$ & $\begin{array}{l}1999 \text { Tax Effort } \\
\text { Index }\end{array}$ \\
\hline Alabama & $4,369,862$ & 167.83 & 772.54 & $1,249.89$ & $1,001.50$ & $3,191.75$ & 78.27 & 17.91 & 156 & 50.38 & 11.53 & 64 \\
\hline Alaska & 619,500 & $4,248.87$ & 24.89 & 624.77 & $1,039.50$ & $5,938.03$ & 145.61 & 235.05 & 2,044 & 426.02 & 687.69 & 293 \\
\hline Arizona & $4,778,332$ & 0.94 & 197.98 & 0.92 & $2,625.00$ & $2,824.84$ & 69.27 & 14.50 & 126 & 0.00 & 0.00 & 0 \\
\hline Arkansas & $2,551,373$ & 101.23 & 0.39 & 717.37 & 490.50 & $1,309.49$ & 32.11 & 12.59 & 109 & 10.24 & 4.01 & 32 \\
\hline California & $33,145,121$ & $3,276.38$ & 0.00 & 762.99 & $3,150.00$ & $7,189.37$ & 176.30 & 5.32 & 46 & 33.74 & 1.02 & 19 \\
\hline Colorado & $4,056,133$ & 300.85 & 514.66 & $1,379.63$ & 612.00 & $2,807.14$ & 68.84 & 16.97 & 148 & 33.98 & 8.38 & 49 \\
\hline Connecticut & $3,282,031$ & 0.00 & 0.00 & 0.00 & 94.35 & 94.35 & 2.31 & 0.70 & 6 & 0.00 & 0.00 & 0 \\
\hline Delaware & 753,538 & 0.00 & 0.00 & 0.00 & 11.15 & 11.15 & 0.27 & 0.36 & 3 & 0.00 & 0.00 & 0 \\
\hline D.C. & 519,000 & 0.00 & 0.00 & 0.00 & 0.00 & 0.00 & 0.00 & 0.00 & 0 & 0.00 & 0.00 & 0 \\
\hline Florida & $15,111,244$ & 70.54 & 0.00 & 0.00 & $1,915.00$ & $1,985.54$ & 48.69 & 3.22 & 28 & 67.10 & 4.44 & 138 \\
\hline Georgia & $7,788,240$ & 0.00 & 0.00 & 0.00 & $1,680.00$ & $1,680.00$ & 41.20 & 5.29 & 46 & 0.00 & 0.00 & 0 \\
\hline Hawaii & $1,185,497$ & 0.00 & 0.00 & 0.00 & 85.45 & 85.45 & 2.10 & 1.77 & 15 & 0.00 & 0.00 & 0 \\
\hline Idaho & $1,251,700$ & 0.00 & 0.00 & 0.00 & 429.50 & 429.50 & 10.53 & 8.41 & 73 & 4.55 & 3.63 & 43 \\
\hline Illinois & $12,128,370$ & 192.53 & 916.91 & 0.00 & 885.50 & $1,994.94$ & 48.92 & 4.03 & 35 & 0.00 & 0.00 & 0 \\
\hline Indiana & $5,942,901$ & 30.80 & 702.01 & 1.58 & 705.00 & $1,439.39$ & 35.30 & 5.94 & 52 & 0.51 & 0.09 & 1 \\
\hline lowa & $2,869,413$ & 0.00 & 0.00 & 0.00 & 503.50 & 503.50 & 12.35 & 4.30 & 37 & 0.00 & 0.00 & 0 \\
\hline Kansas & $2,654,052$ & 464.68 & 6.41 & $1,012.20$ & 575.50 & $2,058.79$ & 50.49 & 19.02 & 165 & 49.28 & 18.57 & 98 \\
\hline Kentucky & $3,960,825$ & 40.19 & $3,430.62$ & 177.35 & 501.00 & $4,149.16$ & 101.75 & 25.69 & 223 & 174.78 & 44.13 & 172 \\
\hline Louisiana & $4,372,035$ & $1,908.78$ & 52.97 & $11,101.93$ & 380.50 & $13,444.18$ & 329.68 & 75.41 & 656 & 350.70 & 80.22 & 106 \\
\hline Maine & $1,253,040$ & 0.00 & 0.00 & 0.00 & 97.30 & 97.30 & 2.39 & 1.90 & 17 & 0.00 & 0.00 & 0 \\
\hline Maryland & $5,171,634$ & 0.00 & 94.07 & 0.11 & 334.00 & 428.18 & 10.50 & 2.03 & 18 & 0.00 & 0.00 & 0 \\
\hline Massachusetts & $6,175,169$ & 0.00 & 0.00 & 0.00 & 198.50 & 198.50 & 4.87 & 0.79 & 7 & 0.00 & 0.00 & 0 \\
\hline Michigan & $9,863,775$ & 120.87 & 0.00 & 491.22 & $1,625.00$ & $2,237.10$ & 54.86 & 5.56 & 48 & 24.21 & 2.45 & 44 \\
\hline Minnesota & $4,775,508$ & 0.00 & 0.00 & 0.00 & $1,580.00$ & $1,580.00$ & 38.75 & 8.11 & 71 & 2.16 & 0.45 & 6 \\
\hline Mississippi & $2,768,619$ & 252.18 & 0.30 & 167.21 & 154.50 & 574.18 & 14.08 & 5.09 & 44 & 19.26 & 6.96 & 137 \\
\hline Missouri & $5,468,338$ & 1.22 & 7.12 & 0.00 & $1,335.00$ & $1,343.35$ & 32.94 & 6.02 & 52 & 0.03 & 0.01 & 0 \\
\hline Montana & 882,779 & 216.51 & 357.97 & 95.51 & 499.50 & $1,169.49$ & 28.68 & 32.49 & 283 & 74.19 & 84.04 & 259 \\
\hline Nebraska & $1,666,028$ & 41.16 & 0.00 & 2.05 & 127.35 & 170.56 & 4.18 & 2.51 & 22 & 0.76 & 0.46 & 18 \\
\hline Nevada & $1,809,253$ & 9.84 & 0.00 & 0.00 & $3,025.00$ & $3,034.84$ & 74.42 & 41.13 & 358 & 33.19 & 18.35 & 45 \\
\hline New Hampshire & $1,201,134$ & 0.00 & 0.00 & 0.00 & 62.00 & 62.00 & 1.52 & 1.27 & 11 & 0.00 & 0.00 & 0 \\
\hline New Jersey & $8,143,412$ & 0.00 & 0.00 & 0.00 & 288.50 & 288.50 & 7.07 & 0.87 & 8 & 0.00 & 0.00 & 0 \\
\hline New Mexico & $1,739,844$ & $1,009.00$ & 601.39 & 2,919.84 & 801.50 & $5,331.73$ & 130.75 & 75.15 & 654 & 269.94 & 155.15 & 206 \\
\hline New York & $18,196,601$ & 3.39 & 0.00 & 38.84 & 984.50 & $1,026.74$ & 25.18 & 1.38 & 12 & 0.00 & 0.00 & 0 \\
\hline North Carolina & $7,650,789$ & 0.00 & 0.00 & 0.00 & 746.00 & 746.00 & 18.29 & 2.39 & 21 & 1.95 & 0.25 & 11 \\
\hline North Dakota & 633,666 & 477.10 & 244.49 & 118.11 & 39.45 & 879.15 & 21.56 & 34.02 & 296 & 100.11 & 157.99 & 464 \\
\hline Ohio & $11,256,654$ & 88.87 & 703.24 & 294.47 & $1,035.00$ & $2,121.58$ & 52.03 & 4.62 & 40 & 12.17 & 1.08 & 23 \\
\hline Oklahoma & $3,358,044$ & $1,120.68$ & 43.78 & $3,068.83$ & 450.50 & $4,683.79$ & 114.86 & 34.20 & 297 & 210.21 & 62.60 & 183 \\
\hline Oregon & $3,316,154$ & 0.00 & 0.00 & 2.89 & 310.00 & 312.89 & 7.67 & 2.31 & 20 & 49.56 & 14.94 & 646 \\
\hline Pennsylvania & $11,994,016$ & 27.11 & $1,970.34$ & 0.00 & $1,230.00$ & $3,227.45$ & 79.14 & 6.60 & 57 & 0.00 & 0.00 & 0 \\
\hline Rhode Island & 990,819 & 0.00 & 0.00 & 0.00 & 23.70 & 23.70 & 0.58 & 0.59 & 5 & 0.00 & 0.00 & 0 \\
\hline South Carolina & $3,885,736$ & 0.00 & 0.00 & 0.00 & 562.00 & 562.00 & 13.78 & 3.55 & 31 & 0.00 & 0.00 & 0 \\
\hline South Dakota & 733,133 & 16.68 & 0.00 & 3.67 & 256.00 & 276.35 & 6.78 & 9.24 & 80 & 3.18 & 4.33 & 47 \\
\hline Tennessee & $5,483,535$ & 4.24 & 83.08 & 2.93 & 737.00 & 827.25 & 20.29 & 3.70 & 32 & 0.80 & 0.15 & 4 \\
\hline Texas & $20,044,141$ & $6,982.24$ & 658.49 & $13,554.35$ & $1,840.00$ & $23,035.08$ & 564.87 & 28.18 & 245 & 705.25 & 35.18 & 125 \\
\hline Utah & $2,129,836$ & 263.94 & 469.32 & 492.42 & $1,305.00$ & $2,530.68$ & 62.06 & 29.14 & 253 & 14.51 & 6.81 & 23 \\
\hline Vermont & 593,740 & 0.00 & 0.00 & 0.00 & 70.70 & 70.70 & 1.73 & 2.92 & 25 & 0.00 & 0.00 & 0 \\
\hline Virginia & $6,872,912$ & 0.09 & 908.77 & 0.00 & 643.00 & $1,551.86$ & 38.06 & 5.54 & 48 & 1.82 & 0.26 & 5 \\
\hline Washington & $5,756,361$ & 0.00 & 75.08 & 0.00 & 635.50 & 710.58 & 17.42 & 3.03 & 26 & 62.32 & 10.83 & 358 \\
\hline West Virginia & $1,806,928$ & 20.88 & $4,336.20$ & 0.00 & 171.50 & $4,528.58$ & 111.05 & 61.46 & 535 & 153.36 & 84.87 & 138 \\
\hline Wisconsin & $5,250,446$ & 0.00 & 0.00 & 0.00 & 335.50 & 335.50 & 8.23 & 1.57 & 14 & 2.46 & 0.47 & 30 \\
\hline Wyoming & 479,602 & 849.96 & $1,757.33$ & $1,486.48$ & $1,035.00$ & $5,128.77$ & 125.77 & 262.24 & 2,281 & 192.44 & 401.25 & 153 \\
\hline Totals & $\overline{272,690,813}$ & $\overline{29,103.80}$ & $\overline{19,023.51}$ & "40,372.09 & $3 \overline{39,350.00}$ & $\overline{127,849.40}$ & $3,135.15$ & 111.50 & 100 & 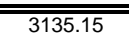 & 111.50 & 100 \\
\hline
\end{tabular}

RTS rate 


\begin{tabular}{|c|c|c|c|c|c|c|c|c|}
\hline State & 1999 State Population & $\begin{array}{c}1999 \text { Total Other Tax Base } \\
\text { (state personal income) } \\
\text { (millions) }\end{array}$ & $\begin{array}{l}1999 \text { Tax Capacity } \\
\text { (\$ millions) }\end{array}$ & $\begin{array}{l}1999 \text { Capacity per } \\
\text { capita } \\
\text { (\$/person) }\end{array}$ & $\begin{array}{l}1999 \text { Per Capita Capacity } \\
\text { Index }\end{array}$ & $\begin{array}{l}1999 \text { Tax } \\
\text { Revenue } \\
\text { (\$ millions) }\end{array}$ & $\begin{array}{c}1999 \text { Revenue per } \\
\text { capita } \\
\text { (\$/person) }\end{array}$ & $\begin{array}{l}1999 \text { Tax Effort } \\
\text { Index }\end{array}$ \\
\hline$\overline{\text { Alabama }}$ & $4,369,862$ & $98,451.58$ & 569.55 & 130.34 & 81 & 637.94 & 145.99 & 112 \\
\hline Alaska & 619,500 & $17,368.92$ & 100.48 & 162.20 & 101 & 67.71 & 109.30 & 67 \\
\hline Arizona & $4,778,332$ & $116,579.55$ & 674.43 & 141.14 & 88 & 347.26 & 72.67 & 51 \\
\hline California & $33,145,121$ & $963,445.14$ & $5,573.64$ & 168.16 & 104 & $5,271.22$ & 159.03 & 95 \\
\hline Colorado & $4,056,133$ & $123,399.65$ & 713.88 & 176.00 & 109 & 372.85 & 91.92 & 52 \\
\hline Connecticut & $3,282,031$ & $127,729.68$ & 738.93 & 225.14 & 140 & 522.67 & 159.25 & 71 \\
\hline Delaware & 753,538 & $22,297.55$ & 128.99 & 171.18 & 106 & 342.34 & 454.32 & 265 \\
\hline D.C. & 519,000 & $20,520.31$ & 118.71 & 228.73 & 142 & 223.29 & 430.23 & 188 \\
\hline Florida & $15,111,244$ & $415,151.37$ & $2,401.70$ & 158.93 & 99 & $3,222.24$ & 213.23 & 134 \\
\hline Hawaii & $1,185,497$ & $32,233.44$ & 186.47 & 157.30 & 98 & 193.87 & 163.53 & 104 \\
\hline Idaho & $1,251,700$ & $27,983.57$ & 161.89 & 129.33 & 80 & 112.84 & 90.15 & 70 \\
\hline Illinois & $12,128,370$ & $367,947.00$ & $2,128.62$ & 175.51 & 109 & $1,537.16$ & 126.74 & 72 \\
\hline Indiana & $5,942,901$ & $151,861.60$ & $\begin{array}{c}878.54 \\
\end{array}$ & 147.83 & 92 & 219.79 & 36.98 & 25 \\
\hline lowa & $2,869,413$ & $72,094.39$ & 417.07 & 145.35 & 90 & 180.66 & 62.96 & 43 \\
\hline Kansas & $2,654,052$ & $68,946.54$ & 398.86 & 150.28 & 93 & 118.71 & 44.73 & 30 \\
\hline Kentucky & $3,960,825$ & $89,683.08$ & 518.83 & 130.99 & 81 & 888.64 & 224.36 & 171 \\
\hline Louisiana & $4,372,035$ & $98,254.72$ & 568.42 & 130.01 & 81 & 602.00 & 137.69 & 106 \\
\hline Maine & $1,253,040$ & $30,074.15$ & 173.98 & 138.85 & 86 & 75.60 & 60.33 & 43 \\
\hline Maryland & $\frac{1,25,1,634}{5,171}$ & $162,930.76$ & 942.57 & 182.26 & 113 & $1,483.68$ & 286.89 & 157 \\
\hline Massachusetts & $6,175,169$ & $211,121.05$ & $1,221.36$ & 197.79 & 123 & 584.16 & 94.60 & 48 \\
\hline Michigan & $9,863,775$ & $270,353.38$ & $1,564.03$ & 158.56 & 98 & 816.04 & 82.73 & 52 \\
\hline Mississippi & $2,768,619$ & $55,935.42$ & 323.59 & 116.88 & 72 & 159.57 & 57.63 & 49 \\
\hline Missouri & $5,468,338$ & $141,400.38$ & 818.02 & 149.59 & 93 & 597.92 & 109.34 & 73 \\
\hline Montana & 882,779 & $19,173.67$ & 110.92 & 125.65 & 78 & 101.22 & 114.66 & 91 \\
\hline Nebraska & $1,666,028$ & $44,303.45$ & 256.30 & 153.84 & 95 & 266.49 & 159.96 & 104 \\
\hline Nevada & $1,809,253$ & $53,727.77$ & 310.82 & 171.80 & 107 & 661.55 & 365.65 & 213 \\
\hline New Hampshire & $1,201,134$ & $36,159.53$ & 209.19 & 174.16 & 108 & 322.61 & 268.59 & 154 \\
\hline New Jersey & $\frac{x, 143,412}{8,143}$ & $283,791.88$ & $1,641.77$ & 201.61 & 125 & 826.20 & 101.46 & 50 \\
\hline New Mexico & $1,739,844$ & $37,291.18$ & 215.73 & 124.00 & 77 & 213.99 & 122.99 & 99 \\
\hline New York & $18,196,601$ & $603,348.79$ & $3,490.44$ & 191.82 & 119 & $4,056.58$ & 222.93 & 116 \\
\hline North Carolina & $7,650,789$ & $197,516.21$ & $1,142.65$ & 149.35 & 93 & $1,050.48$ & 137.30 & 92 \\
\hline North Dakota & 633,666 & $14,778.44$ & 85.50 & 134.92 & 84 & 155.72 & 245.75 & 182 \\
\hline Ohio & $11,256,654$ & $298,775.10$ & $1,728.45$ & 153.55 & 95 & 786.29 & 69.85 & 45 \\
\hline Oklahoma & $3,358,044$ & $76,094.30$ & 440.21 & 131.09 & 81 & 252.43 & 75.17 & 57 \\
\hline Oregon & $3,316,154$ & $87,192.35$ & 504.42 & 152.11 & 94 & 664.91 & 200.51 & 132 \\
\hline Pennsylvania & $11,994,016$ & $336,545.11$ & $1,946.95$ & 162.33 & 101 & $3,155.49$ & 263.09 & 162 \\
\hline Rhode Island & 990,819 & $28,217.15$ & 163.24 & 164.75 & 102 & $\begin{array}{l}114.04 \\
\end{array}$ & 115.10 & 70 \\
\hline South Carolina & $3,885,736$ & $88,873.46$ & 514.14 & 132.32 & 82 & 662.51 & 170.50 & 129 \\
\hline South Dakota & 733,133 & $17,969.53$ & 103.96 & 141.80 & 88 & 145.41 & 198.33 & 140 \\
\hline Tennessee & $5,483,535$ & $137,643.76$ & 796.29 & 145.21 & 90 & 692.04 & 126.20 & 87 \\
\hline Texas & $20,044,141$ & $525,745.46$ & $3,041.50$ & 151.74 & 94 & $4,281.04$ & 213.58 & 141 \\
\hline Utah & $2,129,836$ & $47,847.41$ & 276.80 & 129.96 & 81 & 213.39 & 100.19 & 77 \\
\hline Vermont & 593,740 & $15,156.38$ & 87.68 & 147.68 & 92 & 198.76 & 334.75 & 227 \\
\hline Virginia & $6,872,912$ & $198,867.35$ & $1,150.47$ & 167.39 & 104 & $1,840.19$ & 267.75 & 160 \\
\hline Washington & $5,756,361$ & $168,670.06$ & 975.78 & 169.51 & 105 & $1,483.37$ & 257.69 & 152 \\
\hline
\end{tabular}




\begin{tabular}{|c|c|c|c|c|c|c|c|c|c|c|c|c|c|c|}
\hline & Population & $\begin{array}{c}\text { Primary and } \\
\text { Secondary } \\
\text { Education }\end{array}$ & $\begin{array}{c}\text { Higher } \\
\text { Education }\end{array}$ & $\begin{array}{c}\text { Public } \\
\text { Welfare }\end{array}$ & $\begin{array}{c}\text { Health and } \\
\text { Hospitals }\end{array}$ & Highways & $\begin{array}{l}\text { Police and } \\
\text { Corrections }\end{array}$ & $\begin{array}{c}\text { Environment and } \\
\text { Housing }\end{array}$ & $\begin{array}{c}\text { Interest on } \\
\text { General debt }\end{array}$ & $\begin{array}{c}\text { Governmental } \\
\text { Administration }\end{array}$ & All Other & Total & $\begin{array}{c}\text { Index of } \\
\text { Fiscal } \\
\text { Need } \\
\end{array}$ & Rank \\
\hline Alabama & $4,369,862$ & $1,135.6$ & 439.4 & 945.5 & 510.7 & 445.8 & 384.8 & 391.9 & 246.8 & 263.9 & 546.3 & $5,242.4$ & 103 & 12 \\
\hline Alaska & 619,500 & $1,768.3$ & 530.5 & 553.8 & 373.5 & 301.8 & 488.8 & 416.3 & 246.8 & 301.7 & 598.3 & $5,619.7$ & 110 & 3 \\
\hline Arizona & $4,778,332$ & $1,332.8$ & 430.0 & 916.7 & 437.8 & 324.4 & 394.6 & 396.2 & 246.8 & 270.5 & 555.4 & $5,249.3$ & 103 & 11 \\
\hline Arkansas & $2,551,373$ & $1,174.5$ & 402.2 & 931.5 & 522.5 & 460.2 & 312.5 & 382.0 & 246.8 & 248.4 & 525.0 & $5,124.8$ & 100 & 18 \\
\hline California & $33,145,121$ & $1,381.0$ & 503.0 & 950.8 & 429.6 & 289.7 & 409.7 & 415.8 & 246.8 & 301.0 & 597.3 & $5,482.8$ & 107 & 4 \\
\hline Colorado & $4,056,133$ & $1,312.4$ & 450.6 & 560.9 & 391.2 & 374.7 & 341.4 & 403.3 & 246.8 & 281.5 & 570.5 & $4,979.5$ & 97 & 32 \\
\hline Connecticut & $3,282,031$ & $1,322.9$ & 440.9 & 544.0 & 425.2 & 301.8 & 330.6 & 423.9 & 246.8 & 313.6 & 614.6 & $5,031.9$ & 98 & 26 \\
\hline Delaware & 753,538 & $1,108.6$ & 460.0 & 667.1 & 442.7 & 362.6 & 317.7 & 408.9 & 246.8 & 290.3 & 582.6 & $4,893.5$ & 96 & 39 \\
\hline D.C. & 519,000 & 786.5 & 491.2 & $1,214.1$ & 507.7 & 212.8 & $1,350.2$ & 418.7 & 246.8 & 305.4 & 603.4 & $6,018.9$ & 118 & 1 \\
\hline Florida & $15,111,244$ & $1,056.5$ & 376.0 & $\begin{array}{l}1,216.9 \\
816.9\end{array}$ & 440.8 & 293.7 & 316.4 & 390.7 & 246.8 & 261.9 & 543.7 & $4,702.5$ & 92 & 47 \\
\hline Georgia & $7,788,240$ & $1,287.6$ & 465.6 & 850.1 & 466.6 & 423.9 & 403.2 & 402.0 & 246.8 & 279.4 & 567.7 & $5,345.4$ & 105 & 7 \\
\hline Hawaii & $1,185,497$ & $1,054.1$ & 448.8 & 699.8 & 355.6 & 209.1 & 316.8 & 398.0 & 246.8 & 273.3 & 559.2 & $4,577.5$ & 90 & 51 \\
\hline Idaho & $1,251,700$ & $1,368.2$ & 459.7 & 859.4 & 408.4 & 453.2 & 276.8 & 387.7 & 246.8 & 257.2 & 537.2 & $5,203.9$ & 102 & 15 \\
\hline Illinois & $12,128,370$ & $1,260.0$ & 464.3 & 648.3 & 409.9 & 294.0 & 424.7 & 410.2 & 246.8 & 292.2 & 585.3 & $5,046.8$ & 99 & 25 \\
\hline Indiana & $5,942,901$ & $1,227.5$ & 452.2 & 515.2 & 423.8 & 401.6 & 381.0 & 401.9 & 246.8 & 279.4 & 567.6 & $4,914.2$ & 96 & 38 \\
\hline lowa & $2,869,413$ & $1,144.7$ & 416.8 & 528.6 & 389.1 & 436.8 & 249.5 & 388.2 & 246.8 & 258.1 & 538.4 & $4,594.8$ & 90 & 50 \\
\hline Kansas & $2,654,052$ & $1,234.6$ & 433.7 & 695.4 & 396.6 & 489.7 & 345.8 & 390.3 & 246.8 & 261.3 & 542.8 & $5,014.3$ & 98 & 27 \\
\hline Kentucky & $3,960,825$ & $1,113.9$ & 443.7 & 818.0 & 543.8 & 413.0 & 337.7 & 392.3 & 246.8 & 264.5 & 547.1 & $5,062.8$ & 99 & 24 \\
\hline Louisiana & $4,372,035$ & $1,178.5$ & 449.9 & $1,216.2$ & 482.4 & 317.0 & 437.7 & 388.0 & 246.8 & 257.7 & 537.8 & $5,398.8$ & 106 & 5 \\
\hline Maine & $1,253,040$ & $1,102.0$ & 386.5 & 667.1 & 471.5 & 381.1 & 245.0 & 385.3 & 246.8 & 253.5 & 532.1 & $4,657.8$ & 91 & 48 \\
\hline Maryland & $5,171,634$ & $1,229.6$ & 457.9 & 469.7 & 381.3 & 307.8 & 463.3 & 417.8 & 246.8 & 304.2 & 601.6 & $4,964.0$ & 97 & 35 \\
\hline Massachusetts & $6,175,169$ & $1,194.0$ & 442.8 & 662.9 & 462.4 & 270.5 & 287.1 & 413.2 & 246.8 & 296.9 & 591.7 & $4,880.7$ & 96 & 41 \\
\hline Michigan & $9,863,775$ & $1,370.6$ & 473.7 & 670.0 & 444.8 & 338.0 & 420.5 & 414.4 & 246.8 & 298.7 & 594.2 & $5,289.6$ & 104 & 10 \\
\hline Minnesota & $4,775,508$ & $1,322.1$ & 450.0 & 568.0 & 372.8 & 423.2 & 304.8 & 404.7 & 246.8 & 283.7 & 573.6 & $5,013.3$ & 98 & 28 \\
\hline Mississippi & $2,768,619$ & $1,191.6$ & 431.7 & $1,070.3$ & 527.2 & 446.7 & 360.1 & 381.3 & 246.8 & 247.3 & 523.5 & $5,335.3$ & 104 & 8 \\
\hline Missouri & $5,468,338$ & $1,162.1$ & 417.5 & 683.1 & 436.1 & 429.3 & 350.4 & 391.2 & 246.8 & 262.7 & 544.7 & $4,923.4$ & 96 & 37 \\
\hline Montana & 882,779 & $1,177.6$ & 385.4 & $1,014.5$ & 431.2 & 600.4 & 246.7 & 375.2 & 246.8 & 237.8 & 510.6 & $5,142.4$ & 101 & 17 \\
\hline Nebraska & $1,666,028$ & $1,144.0$ & 419.1 & 737.2 & 377.8 & 514.1 & 287.9 & 385.5 & 246.8 & 253.9 & 532.6 & $4,886.8$ & 96 & 40 \\
\hline Nevada & $1,809,253$ & $1,332.6$ & 411.8 & 700.9 & 415.7 & 355.8 & 415.5 & 400.1 & 246.8 & 276.5 & 563.7 & $5,109.0$ & 100 & 20 \\
\hline New Hampshire & $1,201,134$ & $1,237.9$ & 409.0 & 566.5 & 412.3 & 334.4 & 253.8 & 401.5 & 246.8 & 278.7 & 566.7 & $4,750.5$ & 93 & 46 \\
\hline New Jersey & $8,143,412$ & $1,262.7$ & 462.7 & 538.5 & 423.0 & 262.7 & 348.6 & 426.3 & 246.8 & 317.2 & 619.6 & $4,969.0$ & 97 & 34 \\
\hline New Mexico & $1,739,844$ & $1,343.8$ & 411.7 & $1,301.8$ & 451.6 & 490.1 & 399.6 & 384.7 & 246.8 & 252.5 & 530.7 & $5,707.1$ & 112 & 2 \\
\hline New York & $18,196,601$ & $1,168.0$ & 451.4 & 998.7 & 472.7 & 229.2 & 357.0 & 410.6 & 246.8 & 292.9 & 586.1 & $5,168.0$ & 101 & 16 \\
\hline North Carolina & $7,650,789$ & $1,185.8$ & 418.2 & 881.8 & 488.9 & 373.5 & 363.2 & 392.3 & 246.8 & 264.4 & 547.1 & $5,111.0$ & 100 & 19 \\
\hline North Dakota & 633,666 & $1,152.1$ & 412.2 & 889.3 & 383.7 & 827.8 & 238.4 & 376.3 & 246.8 & 239.6 & 513.0 & $5,216.6$ & 102 & 14 \\
\hline Ohio & $11,256,654$ & $1,231.6$ & 450.8 & 748.6 & 436.5 & 312.7 & 319.2 & 404.5 & 246.8 & 283.4 & 573.1 & $4,994.7$ & 98 & 31 \\
\hline Oklahoma & $3,358,044$ & $1,212.3$ & 411.2 & 849.3 & 428.6 & 477.7 & 340.5 & 382.3 & 246.8 & 248.9 & 525.8 & $5,064.8$ & 99 & 22 \\
\hline Oregon & $3,316,154$ & $1,230.9$ & 431.1 & 886.4 & 421.8 & 378.4 & 287.6 & 398.6 & 246.8 & 274.2 & 560.5 & $5,086.0$ & 100 & 21 \\
\hline Pennsylvania & $11,994,016$ & $1,120.2$ & 424.9 & 665.5 & 426.6 & 286.9 & 341.1 & 406.0 & 246.8 & 285.7 & 576.3 & $4,785.5$ & 94 & 45 \\
\hline Rhode Island & 990,819 & $1,141.4$ & 422.5 & 690.1 & 461.8 & 265.6 & 307.0 & 403.7 & 246.8 & 282.1 & 571.3 & $4,814.2$ & 94 & 44 \\
\hline South Carolina & $3,885,736$ & $1,138.8$ & 435.7 & 809.8 & 479.9 & 383.2 & 354.4 & 389.6 & 246.8 & 260.2 & 541.3 & $5,005.5$ & 98 & 29 \\
\hline South Dakota & 733,133 & $1,163.6$ & 400.4 & 585.8 & 369.1 & 732.2 & 248.6 & 374.7 & 246.8 & 237.1 & 509.5 & $4,860.9$ & 95 & 42 \\
\hline Tennessee & $5,483,535$ & $1,115.2$ & 423.3 & 811.4 & 500.6 & 394.9 & 363.5 & 392.1 & 246.8 & 264.2 & 546.7 & $4,997.9$ & 98 & 30 \\
\hline Texas & $20,044,141$ & $1,397.4$ & 463.3 & 963.1 & 412.5 & 360.9 & 370.3 & 398.2 & 246.8 & 273.6 & 559.7 & $5,380.2$ & 105 & 6 \\
\hline Utah & $2,129,836$ & $1,654.0$ & 584.6 & 470.6 & 352.6 & 377.7 & 344.2 & 403.0 & 246.8 & 281.1 & 570.0 & $5,312.8$ & 104 & 9 \\
\hline Vermont & 593,740 & $1,081.4$ & 398.5 & 623.9 & 428.9 & 415.0 & 264.5 & 387.7 & 246.8 & 257.3 & 537.2 & $4,625.6$ & 91 & 49 \\
\hline Virginia & $6,872,912$ & $1,208.9$ & 476.9 & 543.4 & 425.1 & 355.8 & 378.0 & 407.6 & 246.8 & 288.2 & 579.7 & $4,941.2$ & 97 & 36 \\
\hline Washington & $5,756,361$ & $1,327.3$ & 465.3 & 595.1 & 404.5 & 323.6 & 316.4 & 407.4 & 246.8 & 287.9 & 579.3 & $4,976.6$ & 97 & 33 \\
\hline West Virginia & $1,806,928$ & $1,066.2$ & 408.3 & $1,065.3$ & 556.2 & 366.7 & 292.8 & 382.8 & 246.8 & 249.7 & 526.8 & $5,063.0$ & 99 & 23 \\
\hline Wisconsin & $5,250,446$ & $1,203.4$ & 444.0 & 559.4 & 395.4 & 395.5 & 309.5 & 399.9 & 246.8 & 276.2 & 563.3 & $4,818.1$ & 94 & 43 \\
\hline Wyoming & 479,602 & $1,342.5$ & 445.2 & 706.0 & 402.5 & 671.5 & 276.8 & 386.6 & 246.8 & 255.5 & 534.8 & $5,234.4$ & 102 & 13 \\
\hline $\begin{array}{l}\text { Totals } \\
\end{array}$ & $\overline{272,690,813}$ & $1,1246.4$ & 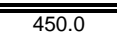 & $\overline{789.1}$ & 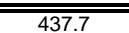 & 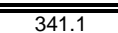 & 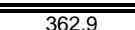 & 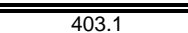 & 246.8 & 281.3 & $\overline{570.2}$ & $\overline{5,108,6}$ & 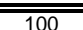 & \\
\hline
\end{tabular}


Direct General Expenditures of State and Local Governments, by function, 1999 (millions)

\begin{tabular}{|c|c|c|c|c|c|c|c|c|c|c|c|}
\hline & $\begin{array}{l}\text { Secondary } \\
\text { Education }\end{array}$ & Higher Education & Public Welfare & $\begin{array}{c}\text { Health and } \\
\text { Hospitals }\end{array}$ & Highways & $\begin{array}{l}\text { Police and } \\
\text { Corrections }\end{array}$ & $\begin{array}{l}\text { Environment and } \\
\text { Housing }\end{array}$ & $\begin{array}{c}\text { Interest on General } \\
\text { debt }\end{array}$ & $\begin{array}{l}\text { Governmental } \\
\text { Administration }\end{array}$ & All Other & Total \\
\hline$\overline{\text { Alabama }}$ & $4,560.3$ & $2,197.5$ & $3,230.9$ & $3,234.2$ & $1,248.5$ & $1,054.3$ & $1,419.5$ & 857.2 & 640.4 & $1,676.4$ & $20,119.1$ \\
\hline Alaska & $1,348.4$ & 341.2 & 689.2 & 250.4 & 748.2 & 332.2 & 594.7 & 482.9 & 395.2 & $1,949.3$ & $7,131.5$ \\
\hline Arizona & $4,874.4$ & $2,265.3$ & $1,884.9$ & 987.5 & $1,737.9$ & $1,885.1$ & $1,773.1$ & $1,423.5$ & 908.2 & $2,562.3$ & $20,302.0$ \\
\hline Arkansas & $2,549.7$ & $1,124.0$ & $1,904.2$ & $1,080.0$ & 859.8 & 618.3 & 664.9 & 508.7 & 346.8 & $1,005.4$ & $10,661.7$ \\
\hline California & $38,850.9$ & $15,526.7$ & $27,133.3$ & $16,763.8$ & $7,383.0$ & $14,661.2$ & $17,106.2$ & $12,292.0$ & $7,654.1$ & $23,503.5$ & $180,874.8$ \\
\hline Colorado & $4,769.0$ & $2,280.7$ & $2,576.4$ & 998.2 & $1,764.9$ & $1,565.5$ & $1,591.9$ & $1,075.4$ & $1,100.6$ & $2,345.2$ & $20,067.9$ \\
\hline Connecticut & $4,813.9$ & $1,112.6$ & $2,930.2$ & $1,546.6$ & $1,106.6$ & $1,182.7$ & $1,265.7$ & $1,274.6$ & $1,290.7$ & $3,164.7$ & $19,688.2$ \\
\hline Delaware & 931.3 & 538.9 & 502.9 & 260.4 & 388.0 & 353.9 & 385.6 & 370.8 & 302.4 & 449.2 & $4,483.3$ \\
\hline D.C. & 694.3 & 82.4 & $1,246.6$ & 509.7 & 101.0 & 605.9 & 381.0 & 388.1 & 253.9 & 366.7 & $4,629.7$ \\
\hline Florida & $15,770.7$ & $4,768.0$ & $8,847.6$ & $6,599.9$ & $5,055.4$ & $6,698.7$ & $7,698.2$ & $4,287.9$ & $3,929.2$ & $7,975.0$ & $71,630.5$ \\
\hline Georgia & $9,796.4$ & $3,136.9$ & $4,920.8$ & $3,668.7$ & $2,476.9$ & $2,469.9$ & $2,638.2$ & $1,985.0$ & $1,089.8$ & $3,648.9$ & $35,831.4$ \\
\hline Hawaii & $1,069.6$ & 686.3 & 956.3 & 548.1 & 403.1 & 355.5 & 627.0 & 439.6 & $\begin{array}{c}1,005.8 \\
595.8\end{array}$ & $1,207.5$ & $\begin{array}{l}6,888.7 \\
6,808\end{array}$ \\
\hline Idaho & $1,377.0$ & 582.5 & 721.2 & 545.4 & 524.5 & 399.1 & 465.6 & 379.0 & 172.6 & 444.7 & $5,611.6$ \\
\hline Illinois & $15,410.1$ & $4,623.5$ & $8,657.1$ & $4,175.7$ & $3,813.7$ & $4,317.6$ & $5,238.3$ & $3,152.4$ & $3,496.1$ & $6,842.3$ & $59,726.8$ \\
\hline Indiana & $6,956.9$ & $3,281.4$ & $3,893.6$ & $2,482.5$ & $1,826.7$ & $1,418.0$ & $1,861.5$ & $1,311.2$ & 900.2 & $2,709.5$ & $26,641.6$ \\
\hline lowa & $3,453.6$ & $\begin{array}{l}0,1,939.8 \\
\end{array}$ & $\frac{0,050}{2,077.6}$ & $\begin{array}{l}2,602.9 \\
1,619.3\end{array}$ & $\frac{1,020.1}{1,607.4}$ & $\frac{1,410.0}{678.9}$ & $\frac{x, 001.1}{980.1}$ & $\frac{1,11.7}{701.7}$ & 313.3 & $\frac{2,0.35}{1,315.1}$ & $\begin{array}{l}14,686.6 \\
\end{array}$ \\
\hline Kansas & $2,961.1$ & $1,636.9$ & $1,296.3$ & $1,005.1$ & $1,339.0$ & 743.1 & 730.6 & 703.6 & 436.9 & $1,213.1$ & $12,065.5$ \\
\hline Kentucky & $3,766.7$ & $1,845.6$ & $3,594.6$ & $1,095.6$ & $1,580.9$ & 944.4 & $1,103.2$ & 865.4 & $1,375.2$ & $1,890.4$ & $18,061.9$ \\
\hline $\begin{array}{l}\text { Louisiana } \\
\text { Lena }\end{array}$ & $4,611.9$ & $1,804.7$ & $2,982.1$ & $\begin{array}{l}1,0077.9 \\
3,077.9\end{array}$ & $1,548.2$ & $1,495.0$ & $1,645.5$ & $1,117.8$ & 932.8 & $1,901.9$ & $21,117.7$ \\
\hline Maine & $1,528.6$ & 446.5 & $1,422.4$ & 393.4 & 512.3 & 263.3 & $\begin{array}{l}465.4 \\
\end{array}$ & 339.3 & 262.8 & 754.1 & $6,388.1$ \\
\hline Maryland & $6,256.6$ & $2,509.9$ & $3,674.6$ & $1,206.1$ & $1,471.1$ & $1,923.7$ & $2,524.4$ & $1,465.2$ & $1,121.2$ & $2,723.0$ & $24,875.8$ \\
\hline Massachusetts & $7,968.7$ & $1,947.0$ & $5,843.0$ & $\begin{array}{l}1,100.1 \\
2,186.4\end{array}$ & $\begin{array}{l}1,711.1 \\
2,720.0\end{array}$ & $\begin{array}{l}1,196.7 \\
2,196\end{array}$ & $\begin{array}{l}2,024.4 \\
3,091.9\end{array}$ & $\begin{array}{l}1,403.2 \\
1,647.4\end{array}$ & $2,283.7$ & $5,114.8$ & $\begin{array}{l}24,099.6 \\
34,996\end{array}$ \\
\hline Michigan & $14,434.2$ & $6,231.8$ & $6,452.0$ & $4,234.6$ & $3,043.0$ & $3,538.5$ & $3,291.3$ & $2,447.9$ & $2,229.7$ & $4,654.5$ & $50,557.6$ \\
\hline Minnesota & $6,835.7$ & $2,444.8$ & $5,501.3$ & $1,725.2$ & $2,222.5$ & $1,369.6$ & $2,462.8$ & $1,586.3$ & $1,469.4$ & $2,542.4$ & $28,160.1$ \\
\hline Mississippi & $\begin{array}{l}0,003.1 \\
2,745.9\end{array}$ & $\begin{array}{l}2,444.0 \\
1,403.0\end{array}$ & $2,040.7$ & $1,928.5$ & $\begin{array}{l}2,142.1 \\
1,142.1\end{array}$ & $\begin{array}{c}1,003.0 \\
678.6\end{array}$ & $\begin{array}{c}2,40<.0 \\
665.1\end{array}$ & $\begin{array}{c}1,000.3 \\
609.2\end{array}$ & $\begin{array}{c}1,4003.4 \\
450.0\end{array}$ & $\begin{array}{l}2,242.4 \\
1,276.8\end{array}$ & $12,939.8$ \\
\hline Missouri & $\frac{2,145.4}{6,017.4}$ & $\frac{1,405.9}{2,150.9}$ & $\frac{2,0409.0}{3,809.0}$ & $\begin{array}{l}1,1,101.8 \\
\end{array}$ & $\begin{array}{l}1,142 . .1 \\
1,922.6\end{array}$ & $1,501.1$ & $1,391.6$ & $1,283.1$ & 934.3 & $\frac{1,70.0}{2,768.8}$ & $23,880.6$ \\
\hline Montana & $1,016.5$ & 474.8 & 511.0 & 298.8 & 481.1 & 242.2 & 368.5 & 292.4 & 226.3 & 381.0 & $4,292.6$ \\
\hline Nebraska & $1,968.3$ & $1,052.2$ & $1,129.8$ & 542.6 & 801.6 & 393.0 & 577.4 & 358.0 & 211.9 & 681.4 & $7,716.0$ \\
\hline Nevada & $2,194.9$ & 683.1 & 852.1 & 674.7 & 959.1 & 869.0 & 793.8 & 781.9 & 623.2 & 864.1 & $9,296.0$ \\
\hline New Hampshire & $1,370.7$ & 418.3 & 986.4 & 156.2 & 458.0 & 283.4 & 338.5 & 303.1 & 399.6 & 614.8 & $5,328.9$ \\
\hline New Jersey & $13,378.1$ & $3,088.8$ & $5,701.2$ & $2,071.8$ & $2,390.7$ & $3,397.5$ & $3,460.2$ & $2,565.1$ & $2,297.2$ & $5,974.8$ & $44,325.5$ \\
\hline New Mexico & $2,049.4$ & $1,144.4$ & $1,381.7$ & 846.0 & $1,132.3$ & 646.5 & 663.3 & 522.6 & 366.8 & 712.6 & $9,465.6$ \\
\hline New York & $30,699.9$ & $5,735.7$ & $28,052.7$ & $11,017.9$ & $6,555.6$ & $9,803.3$ & $8,756.6$ & $6,215.2$ & $8,536.6$ & $18,395.8$ & $133,769.3$ \\
\hline North Carolina & $8,313.3$ & $4,122.2$ & $5,691.1$ & $5,298.4$ & $2,512.1$ & $2,357.4$ & $2,771.7$ & $1,478.9$ & $1,122.5$ & $3,101.9$ & $36,769.3$ \\
\hline North Dakota & 702.5 & 436.3 & 622.0 & 51.9 & 412.7 & 113.7 & 385.7 & 160.3 & 127.1 & $\begin{array}{c}0,101.7 \\
474.7\end{array}$ & $3,486.8$ \\
\hline Ohio & $13,555.5$ & $4,672.1$ & $8,848.1$ & $4,471.8$ & $3,555.2$ & $\frac{1.1 .1}{3,731.0}$ & $4,117.8$ & $3,290.8$ & $1,909.7$ & $4,998.0$ & $53,150.1$ \\
\hline Oklahoma & $3,675.4$ & $1,610.2$ & $1,738.9$ & $1,260.6$ & $1,122.9$ & 859.2 & 928.2 & 692.1 & 465.1 & $1,202.4$ & $13,555.0$ \\
\hline Oregon & $4,045.0$ & $1,937.3$ & $2,771.0$ & $1,452.5$ & $1,239.7$ & $1,330.6$ & $1,696.4$ & $1,282.8$ & 616.0 & $1,929.3$ & $18,300.8$ \\
\hline Pennsylvania & $15,983.6$ & $4,666.8$ & $11,310.5$ & $\begin{array}{l}1,4011.1 \\
3,611\end{array}$ & $3,781.7$ & $4,235.1$ & $4,236.3$ & $\begin{array}{l}1,941.0 \\
2,940\end{array}$ & $4,062.2$ & $6,397.3$ & $61,225.5$ \\
\hline Rhode Island & $1,273.8$ & 368.9 & $1,042.3$ & 242.1 & 298.2 & 328.5 & 383.2 & 305.7 & 318.0 & 720.2 & $5,280.8$ \\
\hline South Carolina & $4,477.2$ & $1,737.5$ & $3,131.4$ & $2,826.1$ & $1,034.1$ & $1,151.5$ & $1,048.6$ & 815.6 & 641.6 & $1,368.7$ & $18,232.2$ \\
\hline South Dakota & 808.3 & 289.3 & 446.1 & 151.6 & 472.6 & 165.2 & 268.2 & 188.7 & 155.3 & 300.2 & $3,245.4$ \\
\hline Tennessee & $5,531.9$ & $2,204.6$ & $4,324.3$ & $2,953.2$ & $1,689.3$ & $\begin{array}{l}1,439.9 \\
\end{array}$ & $1,894.8$ & $1,116.4$ & 827.0 & $2,190.7$ & $24,172.1$ \\
\hline Texas & $25,404.8$ & $8,978.4$ & $11,259.5$ & $8,198.4$ & $5,854.6$ & $6,831.4$ & $5,597.6$ & $3,870.1$ & $4,063.7$ & $7,316.0$ & $87,374.5$ \\
\hline Utah & $\begin{array}{l}2,4084.0 \\
2,384.0\end{array}$ & $\begin{array}{l}0,510.4 \\
1,527.6\end{array}$ & $\begin{array}{l}1,25.5 \\
1,348.6\end{array}$ & $\begin{array}{l}0,190.4 \\
721.7\end{array}$ & $\begin{array}{l}8,034.0 \\
875.2\end{array}$ & $\begin{array}{l}0,001.4 \\
714.1\end{array}$ & $\begin{array}{l}785.4 \\
\text {. }\end{array}$ & $\begin{array}{l}3,009.1 \\
80.1\end{array}$ & $\begin{array}{c}4,003.1 \\
383.3\end{array}$ & $\begin{array}{l}7,010.8 \\
890.8\end{array}$ & $\begin{array}{l}10,439.7 \\
10.74\end{array}$ \\
\hline Vermont & 821.9 & 340.4 & $\frac{1,040.0}{549.8}$ & 61.2 & 316.2 & 101.5 & 215.0 & 193.9 & $\begin{array}{l}146.9 \\
14.9\end{array}$ & 282.9 & $3,029.7$ \\
\hline Virginia & $8,161.8$ & $3,140.0$ & $4,046.0$ & $2,432.5$ & $2,710.0$ & $2,267.8$ & $2,544.2$ & $1,941.7$ & $1,734.9$ & $3,319.9$ & $32,298.7$ \\
\hline Washington & $7,336.4$ & $3,165.8$ & $4,608.8$ & $3,221.1$ & $2,197.1$ & $1,971.1$ & $3,117.5$ & $1,480.3$ & $1,356.6$ & $3,879.1$ & $32,333.8$ \\
\hline West Virginia & $2,074.0$ & 769.4 & $1,668.8$ & 517.2 & 837.3 & 311.5 & 458.9 & $\begin{array}{c}1,4002.4 \\
492.4\end{array}$ & 407.9 & 689.5 & $8,226.9$ \\
\hline Wisconsin & $7,566.6$ & $2,949.4$ & $4,128.2$ & $1,608.8$ & $2,386.6$ & $1,981.2$ & $2,209.2$ & $1,397.9$ & $1,282.9$ & $2,532.3$ & $28,043.3$ \\
\hline Wyoming & 724.3 & $\begin{array}{c}294.3 \\
2.34\end{array}$ & 250.7 & 448.1 & 397.1 & 188.5 & 249.2 & 208.2 & $\begin{array}{l}126.4 \\
126\end{array}$ & 265.2 & $3,152.1$ \\
\hline United States & $339,871.2$ & $122,716.4$ & $215,189.7$ & $119,361.2$ & $93,018.0$ & 98.964 .6 & $109,929.6$ & $7 \overline{76.699 .2}$ & $67,293.7$ & 155.489 .4 & 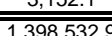 \\
\hline
\end{tabular}




\begin{tabular}{|c|c|c|c|c|c|c|c|c|c|c|c|c|}
\hline & Population & $\begin{array}{l}\text { Primary and } \\
\text { Secondary } \\
\text { Education }\end{array}$ & $\begin{array}{c}\text { Higher } \\
\text { Education }\end{array}$ & Public Welfare & $\begin{array}{c}\text { Health and } \\
\text { Hospitals }\end{array}$ & Highways & $\begin{array}{l}\text { Police and } \\
\text { Corrections }\end{array}$ & $\begin{array}{l}\text { Environment and } \\
\text { Housing }\end{array}$ & $\begin{array}{l}\text { Interest on } \\
\text { General debt }\end{array}$ & $\begin{array}{l}\text { Governmental } \\
\text { Administration }\end{array}$ & All Other & Total \\
\hline Alabama & $4,369,862$ & $1,043.6$ & 502.9 & 739.4 & 740.1 & 285.7 & 241.3 & 324.8 & 146.5 & 196.2 & 383.6 & $4,604.1$ \\
\hline Alaska & 619,500 & $2,176.5$ & 550.7 & $1,112.5$ & 404.2 & $1,207.7$ & 536.3 & 959.9 & 637.9 & 779.5 & 3146.5 & $11,511.7$ \\
\hline Arizona & $4,778,332$ & $1,020.1$ & 474.1 & 394.5 & 206.7 & 363.7 & 394.5 & 371.1 & 190.1 & 297.9 & 536.2 & $4,248.8$ \\
\hline Arkansas & $2,551,373$ & 999.3 & 440.5 & 746.3 & 423.3 & 337.0 & 242.3 & 260.6 & 135.9 & 199.4 & 394.1 & $4,178.8$ \\
\hline California & $33,145,121$ & $1,172.1$ & 468.4 & 818.6 & 505.8 & 222.7 & 442.3 & 516.1 & 230.9 & 370.9 & 709.1 & $5,457.1$ \\
\hline Colorado & $4,056,133$ & $1,175.7$ & 562.3 & 635.2 & 246.1 & 435.1 & 386.0 & 392.5 & 271.4 & 265.1 & 578.2 & $4,947.5$ \\
\hline Connecticut & $3,282,031$ & $1,466.7$ & 339.0 & 892.8 & 471.2 & 337.2 & 360.3 & 385.7 & 393.3 & 388.4 & 964.2 & $5,998.8$ \\
\hline Delaware & 753,538 & $1,235.9$ & 715.2 & 667.5 & 345.5 & 514.9 & 469.6 & 511.7 & 401.3 & 492.0 & 596.2 & $5,949.7$ \\
\hline D.C. & 519,000 & $1,337.9$ & 158.8 & $2,401.9$ & 982.2 & 194.5 & $1,167.5$ & 734.1 & 489.2 & 747.7 & 706.6 & $8,920.4$ \\
\hline Florida & $15,111,244$ & $1,043.6$ & 315.5 & 585.5 & 436.8 & 334.5 & 443.3 & 509.4 & 260.0 & 283.8 & 527.8 & $4,740.2$ \\
\hline Georgia & $7,788,240$ & $1,257.8$ & 402.8 & 631.8 & 471.1 & 318.0 & 317.1 & 338.7 & 139.9 & 254.9 & 468.5 & $4,600.7$ \\
\hline Hawaii & $1,185,497$ & 902.2 & 578.9 & 806.7 & 462.3 & 340.0 & 299.8 & 528.9 & 502.5 & 370.8 & 1018.6 & $5,810.8$ \\
\hline Idaho & $1,251,700$ & $1,100.1$ & 465.4 & 576.2 & 435.7 & 419.1 & 318.9 & 372.0 & 137.9 & 302.8 & 355.3 & $4,483.2$ \\
\hline Illinois & $12,128,370$ & $1,270.6$ & 381.2 & 713.8 & 344.3 & 314.4 & 356.0 & 431.9 & 288.3 & 259.9 & 564.2 & $4,924.6$ \\
\hline Indiana & $5,942,901$ & $1,170.6$ & 552.2 & 655.2 & 417.7 & 307.4 & 238.6 & 313.2 & 151.5 & 220.6 & 455.9 & $4,482.9$ \\
\hline lowa & $2,869,413$ & $\frac{1,1303.6}{1,203.6}$ & 676.0 & 724.1 & 564.3 & 560.2 & 236.6 & 341.6 & 109.2 & 244.5 & 458.3 & $5,118.3$ \\
\hline Kansas & $2,654,052$ & $1,115.7$ & 616.7 & 488.4 & 378.7 & 504.5 & 280.0 & 275.3 & 164.6 & 265.1 & 457.1 & $4,546.1$ \\
\hline Kentucky & $3,960,825$ & 951.0 & 466.0 & 907.5 & 276.6 & 399.1 & 238.4 & 278.5 & 347.2 & 218.5 & 477.3 & $4,560.1$ \\
\hline Louisiana & $4,372,035$ & $1,054.9$ & 412.8 & 682.1 & 704.0 & 354.1 & 342.0 & 376.4 & 213.4 & 255.7 & 435.0 & $4,830.2$ \\
\hline Maine & $1,253,040$ & $1,219.9$ & 356.3 & $1,135.1$ & 314.0 & 408.9 & 210.1 & 371.4 & 209.7 & 270.8 & 601.8 & $5,098.1$ \\
\hline Maryland & $5,171,634$ & $1,209.8$ & 485.3 & 710.5 & 233.2 & 284.5 & 372.0 & 488.1 & 216.8 & 283.3 & 526.5 & $4,810.1$ \\
\hline Massachusetts & $6,175,169$ & $1,290.4$ & 315.3 & 946.2 & 354.1 & 440.5 & 355.7 & 500.7 & 369.8 & 266.8 & 828.3 & $5,667.8$ \\
\hline Michigan & $9,863,775$ & $1,463.4$ & 631.8 & 654.1 & 429.3 & 308.5 & 358.7 & 333.7 & 226.0 & 248.2 & 471.9 & $5,125.6$ \\
\hline Minnesota & $4,775,508$ & $1,431.4$ & 511.9 & $1,152.0$ & 361.3 & 465.4 & 286.8 & 515.7 & 307.7 & 332.2 & 532.4 & $5,896.8$ \\
\hline Mississippi & $2,768,619$ & 991.8 & 506.8 & 737.1 & 696.6 & 412.5 & 245.1 & 240.2 & 162.5 & 220.0 & 461.2 & $4,673.7$ \\
\hline Missouri & $5,468,338$ & $1,100.4$ & 393.3 & 696.6 & 384.4 & 351.6 & 274.5 & 254.5 & 170.8 & 234.6 & 506.3 & $4,367.1$ \\
\hline Montana & 882,779 & $1,151.5$ & 537.8 & 578.9 & 338.5 & 545.0 & 274.4 & 417.4 & 256.4 & 331.2 & 431.6 & $4,862.6$ \\
\hline Nebraska & $1,666,028$ & $1,181.5$ & 631.6 & 678.1 & 325.7 & 481.1 & 235.9 & 346.5 & 127.2 & 214.9 & 409.0 & $4,631.4$ \\
\hline Nevada & $1,809,253$ & $1,213.2$ & 377.6 & 471.0 & 372.9 & 530.1 & 480.3 & 438.7 & 344.5 & 432.2 & 477.6 & $5,138.0$ \\
\hline New Hampshire & $1,201,134$ & $1,141.2$ & 348.2 & 821.3 & 130.0 & 381.3 & 235.9 & 281.8 & 332.7 & 252.3 & 511.9 & $4,436.6$ \\
\hline New Jersey & $8,143,412$ & $1,642.8$ & 379.3 & 700.1 & 254.4 & 293.6 & 417.2 & 424.9 & 282.1 & 315.0 & 733.7 & $5,443.1$ \\
\hline New Mexico & $1,739,844$ & $1,177.9$ & 657.8 & 794.2 & 486.3 & 650.8 & 371.6 & 381.2 & 210.8 & 300.4 & 409.6 & $5,440.5$ \\
\hline New York & $18,196,601$ & $1,687.1$ & 315.2 & $1,541.6$ & 605.5 & 360.3 & 538.7 & 481.2 & 469.1 & 341.6 & 1010.9 & $7,351.3$ \\
\hline North Carolina & $7,650,789$ & $1,086.6$ & 538.8 & 743.9 & 692.5 & 328.3 & 308.1 & 362.3 & 146.7 & 193.3 & 405.4 & $4,806.0$ \\
\hline North Dakota & 633,666 & $1,108.7$ & 688.6 & 981.5 & 81.9 & 651.3 & 179.5 & 608.7 & 200.6 & 252.9 & 749.1 & $5,502.7$ \\
\hline Ohio & $11,256,654$ & $\frac{1,100.1}{1,204.2}$ & 415.1 & 786.0 & 397.3 & 315.8 & 331.4 & 365.8 & 169.6 & 292.3 & 444.0 & $\frac{0,00<.1}{4,721.7}$ \\
\hline Oklahoma & $3,358,044$ & $1,094.5$ & 479.5 & 517.8 & 375.4 & 334.4 & 255.9 & 276.4 & 138.5 & 206.1 & 358.1 & $4,036.6$ \\
\hline Oregon & $3,316,154$ & $1,219.8$ & 584.2 & 835.6 & 438.0 & 373.8 & 401.2 & 511.6 & 185.8 & 386.8 & 581.8 & $5,518.7$ \\
\hline Pennsylvania & $11,994,016$ & $1,332.6$ & 389.1 & 943.0 & 301.1 & 315.3 & 353.1 & 353.2 & 338.7 & 245.2 & 533.4 & $5,104.7$ \\
\hline Rhode Island & $\begin{array}{c}990,819 \\
\end{array}$ & $1,285.6$ & 372.3 & $1,051.9$ & 244.3 & 301.0 & 331.5 & 386.7 & 320.9 & 308.5 & 726.9 & $5,329.7$ \\
\hline South Carolina & $3,885,736$ & $1,152.2$ & 447.1 & 805.9 & 727.3 & 266.1 & 296.3 & 269.8 & 165.1 & 209.9 & 352.2 & $4,692.1$ \\
\hline South Dakota & 733,133 & $1,102.6$ & 394.7 & 608.5 & 206.8 & 644.6 & 225.3 & 365.8 & 211.8 & 257.3 & 409.4 & $4,426.8$ \\
\hline Tennessee & $5,483,535$ & $1,008.8$ & 402.0 & 788.6 & 538.6 & 308.1 & 262.6 & 345.6 & 150.8 & 203.6 & 399.5 & $4,408.1$ \\
\hline Texas & $20,044,141$ & $1,267.4$ & 447.9 & 561.7 & 409.0 & 292.1 & 340.8 & 279.3 & 202.7 & 193.1 & 365.0 & $4,359.1$ \\
\hline Utah & $2,129,836$ & $1,119.3$ & 717.2 & 633.2 & 338.9 & 410.9 & 335.3 & 368.8 & 180.0 & 379.9 & 418.3 & $4,901.7$ \\
\hline Vermont & $\frac{2,120,740}{593,740}$ & $\frac{1,1.4 .0}{1,384.3}$ & 573.3 & 925.9 & $\frac{03.0}{103.0}$ & 532.6 & 170.9 & 362.2 & 247.4 & 326.5 & 476.5 & $\frac{4,102.7}{5,102.7}$ \\
\hline $\begin{array}{l}\text { Virginia } \\
\text {. }\end{array}$ & $6,872,912$ & $1,187.5$ & 456.9 & 588.7 & 353.9 & 394.3 & 330.0 & 370.2 & 252.4 & 282.5 & 483.0 & $4,699.4$ \\
\hline Washington & $5,756,361$ & $1,274.5$ & 550.0 & 800.6 & 559.6 & 381.7 & 342.4 & 541.6 & 235.7 & 257.2 & 673.9 & $5,617.1$ \\
\hline West Virginia & $1,806,928$ & $1,147.8$ & 425.8 & 923.5 & 286.2 & 463.4 & 172.4 & 254.0 & 225.7 & 272.5 & 381.6 & $4,553.0$ \\
\hline Wisconsin & $\begin{array}{l}5,250,446 \\
\end{array}$ & $1,441.1$ & 561.7 & 786.3 & 306.4 & 454.5 & 377.3 & 420.8 & 244.3 & 266.3 & 482.3 & $5,341.1$ \\
\hline Wyoming & 479,602 & $1,510.2$ & 613.6 & 522.8 & 934.4 & 828.1 & 393.1 & 519.5 & 263.5 & 434.0 & 553.1 & $6,572.2$ \\
\hline Totals & $\overline{272,690,813}$ & 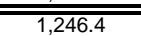 & $4 \quad 450.0$ & $\bar{~} 789.1$ & "437.7 & 2341.1 & 362.9 & 403.1 & 246.8 & 281.3 & "570.2 & $\overline{5,128.6}$ \\
\hline
\end{tabular}


Representative State-Local Expenditures by Function, 1999, Adjusted for Input Cost Differences

\begin{tabular}{|c|c|c|c|c|c|c|c|c|c|c|c|}
\hline & $\begin{array}{l}\text { Primary and } \\
\text { Secondary } \\
\text { Education }\end{array}$ & $\begin{array}{c}\text { Higher } \\
\text { Education }\end{array}$ & Public Welfare & $\begin{array}{c}\text { Health and } \\
\text { Hospitals }\end{array}$ & Highways & $\begin{array}{l}\text { Police and } \\
\text { Corrections }\end{array}$ & $\begin{array}{l}\text { Environment and } \\
\text { Housing }\end{array}$ & $\begin{array}{c}\text { Interest on General } \\
\text { debt }\end{array}$ & $\begin{array}{l}\text { Governmental } \\
\text { Administration }\end{array}$ & All Other & Total \\
\hline Alabama & $4,934.6$ & $1,911.1$ & $4,109.5$ & $2,218.6$ & $1,936.2$ & $1,671.2$ & $1,709.7$ & $1,078.4$ & $1,148.6$ & $2,380.9$ & $23,098.7$ \\
\hline Alaska & $1,089.3$ & 327.1 & 341.2 & 230.0 & 185.8 & 301.0 & 257.4 & 152.9 & 186.2 & 369.6 & $3,440.5$ \\
\hline Arizona & $6,332.6$ & $2,045.2$ & $4,356.9$ & $2,079.7$ & $1,540.8$ & $1,873.8$ & $1,889.9$ & $1,179.2$ & $1,287.6$ & $2,647.0$ & $25,232.7$ \\
\hline Arkansas & $2,979.9$ & $1,021.5$ & $2,363.8$ & $1,325.2$ & $1,167.1$ & 792.4 & 972.8 & 629.6 & 631.2 & $1,335.9$ & $13,219.5$ \\
\hline California & $45,514.7$ & $16,593.5$ & $31,347.2$ & $14,153.9$ & $9,542.5$ & $13,497.3$ & $13,758.7$ & $8,179.4$ & $9,938.5$ & $19,746.6$ & $182,272.3$ \\
\hline Colorado & $5,293.2$ & $1,819.0$ & $2,262.9$ & $1,577.6$ & $1,510.6$ & $1,376.1$ & $1,632.8$ & $1,001.0$ & $1,137.2$ & $2,307.8$ & $19,918.3$ \\
\hline Connecticut & $4,317.4$ & $1,440.1$ & $1,776.0$ & $1,387.3$ & 984.6 & $1,078.5$ & $1,388.9$ & 809.9 & $1,025.3$ & $2,011.9$ & $16,219.9$ \\
\hline Delaware & 830.7 & 345.0 & 500.0 & 331.6 & 271.5 & 237.9 & 307.6 & 186.0 & 217.9 & 437.8 & $3,666.0$ \\
\hline D.C. & 405.9 & 253.8 & 626.7 & 261.9 & 109.8 & 696.5 & 216.9 & 128.1 & 157.9 & 312.3 & $3,169.8$ \\
\hline Florida & $15,875.7$ & $5,654.7$ & $12,278.1$ & $6,622.0$ & $4,410.7$ & $4,752.2$ & $5,893.6$ & $3,729.1$ & $3,942.8$ & $8,193.6$ & $71,352.5$ \\
\hline Georgia & $9,971.9$ & $3,609.4$ & $6,585.7$ & $3,612.6$ & $3,281.1$ & $3,121.3$ & $3,125.0$ & $1,922.0$ & $2,167.9$ & $4,409.7$ & $41,806.5$ \\
\hline Hawaii & $1,242.7$ & 529.6 & 825.1 & 419.1 & 246.4 & 373.2 & 471.0 & 292.6 & 322.7 & 661.2 & $5,383.6$ \\
\hline Idaho & $1,702.9$ & 572.7 & $1,070.0$ & 508.2 & 563.8 & 344.4 & 484.4 & 308.9 & 320.7 & 670.6 & $6,546.6$ \\
\hline Illinois & $15,195.7$ & $5,604.5$ & $7,821.0$ & $4,942.3$ & $3,544.3$ & $5,119.3$ & $4,966.1$ & $2,993.0$ & $3,530.5$ & $7,079.6$ & $60,796.4$ \\
\hline Indiana & $7,253.8$ & $2,674.9$ & $3,045.5$ & $2,504.1$ & $2,372.0$ & $2,250.2$ & $2,384.3$ & $1,466.6$ & $1,653.8$ & $3,364.2$ & $28,969.4$ \\
\hline lowa & $3,266.2$ & $1,190.5$ & $1,508.7$ & $1,110.0$ & $1,245.7$ & 711.5 & $1,112.0$ & 708.1 & 737.7 & $1,540.7$ & $13,131.1$ \\
\hline Kansas & $3,258.2$ & $1,145.6$ & $1,835.7$ & $1,046.4$ & $1,291.7$ & 912.2 & $1,034.0$ & 655.0 & 690.8 & $1,436.8$ & $13,306.4$ \\
\hline Kentucky & $4,387.3$ & $1,749.0$ & $3,222.5$ & $2,141.3$ & $1,625.9$ & $1,329.3$ & $1,551.2$ & 977.4 & $1,043.4$ & $2,161.3$ & $20,188.6$ \\
\hline Louisiana & $5,123.2$ & $1,957.9$ & $5,289.0$ & $2,096.7$ & $1,377.7$ & $1,902.0$ & $1,693.2$ & $1,078.9$ & $1,122.3$ & $2,345.1$ & $23,986.0$ \\
\hline Maine & $1,373.0$ & 482.0 & 831.5 & 587.3 & 474.6 & 305.1 & 481.9 & 309.2 & 316.4 & 665.0 & $5,826.1$ \\
\hline Maryland & $6,323.3$ & $2,357.1$ & $2,415.9$ & $1,960.5$ & $1,582.2$ & $2,381.3$ & $2,157.2$ & $1,276.2$ & $1,566.8$ & $3,103.2$ & $25,123.7$ \\
\hline Massachusetts & $7,331.6$ & $2,721.6$ & $4,071.6$ & $2,838.5$ & $1,660.3$ & $1,762.1$ & $2,547.1$ & $1,523.9$ & $1,826.4$ & $3,644.2$ & $29,927.2$ \\
\hline Michigan & $13,443.4$ & $4,650.9$ & $6,573.5$ & $4,361.5$ & $3,313.3$ & $4,122.0$ & $4,080.0$ & $2,434.1$ & $2,935.1$ & $5,845.5$ & $51,759.2$ \\
\hline Minnesota & $6,278.3$ & $2,138.8$ & $2,698.1$ & $1,769.8$ & $2,008.8$ & $1,446.6$ & $1,929.3$ & $1,178.5$ & $1,349.6$ & $2,731.9$ & $23,529.6$ \\
\hline Mississippi & $3,280.5$ & $1,189.6$ & $2,947.3$ & $1,450.9$ & $1,229.3$ & 990.8 & $1,053.8$ & 683.2 & 682.0 & $1,445.7$ & $14,953.1$ \\
\hline Missouri & $6,318.9$ & $2,272.6$ & $3,715.6$ & $2,370.5$ & $2,333.1$ & $1,904.3$ & $2,135.4$ & $1,349.5$ & $1,431.0$ & $2,970.8$ & $26,801.6$ \\
\hline Montana & $1,033.7$ & 338.6 & 890.8 & 378.4 & 526.8 & 216.5 & 330.6 & 217.8 & 209.1 & 449.5 & $4,591.9$ \\
\hline Nebraska & $1,895.3$ & 695.0 & $1,221.6$ & 625.8 & 851.3 & 476.8 & 641.2 & 411.1 & 421.4 & 885.0 & $8,124.5$ \\
\hline Nevada & $2,397.5$ & 741.5 & $1,261.4$ & 747.7 & 639.7 & 747.2 & 722.6 & 446.5 & 498.4 & $1,017.2$ & $9,219.6$ \\
\hline New Hampshire & $1,478.6$ & 488.9 & 676.8 & 492.3 & 399.2 & 303.0 & 481.4 & 296.4 & 333.4 & $\begin{array}{c}678.8 \\
678.8\end{array}$ & $5,628.9$ \\
\hline New Jersey & $10,224.7$ & $3,750.1$ & $4,361.6$ & $3,424.1$ & $2,126.6$ & $2,821.7$ & $3,465.1$ & $2,009.6$ & $2,573.2$ & $5,032.4$ & $39,789.2$ \\
\hline New Mexico & $2,324.8$ & 712.9 & $2,252.9$ & 781.1 & 847.4 & 691.0 & 668.1 & 429.4 & 437.7 & 921.0 & $10,066.2$ \\
\hline New York & $21,134.6$ & $8,176.0$ & $18,076.4$ & $8,550.5$ & $4,144.5$ & $6,455.9$ & $7,458.1$ & $4,490.5$ & $5,308.2$ & $10,637.3$ & $94,432.2$ \\
\hline North Carolina & $9,021.5$ & $3,184.2$ & $6,710.7$ & $3,718.9$ & $2,840.1$ & $2,761.7$ & $2,996.1$ & $1,888.0$ & $2,015.1$ & $4,174.4$ & $39,310.7$ \\
\hline North Dakota & 725.9 & 260.0 & 560.5 & 241.7 & 521.4 & 150.1 & 238.1 & 156.4 & 151.3 & 324.2 & $3,329.6$ \\
\hline Ohio & $13,785.8$ & $5,050.9$ & $8,381.7$ & $4,884.4$ & $3,498.4$ & $3,571.2$ & $4,545.0$ & $2,777.9$ & $3,177.1$ & $6,433.7$ & $56,106.0$ \\
\hline Oklahoma & $4,047.9$ & $1,374.4$ & $2,836.9$ & $1,430.7$ & $1,594.3$ & $1,136.4$ & $1,281.6$ & 828.7 & 832.6 & $1,760.9$ & $17,124.3$ \\
\hline Oregon & $4,058.9$ & $1,422.9$ & $2,923.6$ & $1,390.5$ & $1,247.3$ & 948.0 & $1,319.5$ & 818.3 & 905.8 & $1,853.8$ & $16,888.6$ \\
\hline Pennsylvania & $13,360.2$ & $5,072.4$ & $7,939.5$ & $5,086.2$ & $3,420.3$ & $4,066.6$ & $4,860.6$ & $2,959.8$ & $3,413.1$ & $6,893.5$ & $57,072.1$ \\
\hline Rhode Island & $1,124.6$ & 416.7 & 680.1 & 454.8 & 261.6 & 302.4 & 399.2 & 244.5 & 278.4 & 564.6 & $4,726.8$ \\
\hline South Carolina & $4,400.0$ & $1,685.2$ & $3,129.7$ & $1,853.8$ & $1,480.0$ & $1,368.6$ & $1,511.2$ & 958.9 & $1,007.2$ & $2,097.8$ & $19,492.5$ \\
\hline South Dakota & 848.3 & 292.1 & 427.2 & 269.0 & 533.5 & 181.2 & 274.2 & 180.9 & 173.1 & 372.6 & $3,552.1$ \\
\hline Tennessee & $6,081.0$ & $2,310.2$ & $4,425.8$ & $2,729.1$ & $2,152.3$ & $1,980.9$ & $2,146.4$ & $1,353.2$ & $1,442.8$ & $2,989.8$ & $27,611.6$ \\
\hline Texas & $27,852.0$ & $9,243.3$ & $19,201.9$ & $8,220.1$ & $7,189.0$ & $7,377.8$ & $7,967.5$ & 4,946.4 & $5,462.7$ & $11,188.4$ & $108,649.0$ \\
\hline Utah & $3,502.9$ & $1,239.2$ & 996.8 & 746.6 & 799.5 & 728.7 & 856.8 & 525.6 & 596.3 & $1,210.7$ & $11,203.2$ \\
\hline Vermont & 638.4 & 235.5 & 368.4 & 253.1 & 244.9 & 156.1 & 229.8 & 146.5 & 152.1 & 318.1 & $\frac{1,200.2}{2,743.0}$ \\
\hline Virginia & $8,261.7$ & $3,262.1$ & $3,715.1$ & $2,904.6$ & $2,430.7$ & $2,581.7$ & $2,796.2$ & $1,696.1$ & $1,972.8$ & $3,973.5$ & $33,594.4$ \\
\hline Washington & $7,597.4$ & $2,665.7$ & $3,407.5$ & $2,315.0$ & $1,851.4$ & $1,810.1$ & $2,341.1$ & $1,420.5$ & $1,650.9$ & $3,326.1$ & $28,385.7$ \\
\hline West Virginia & $1,915.8$ & 734.3 & $1,914.6$ & 999.0 & 658.5 & 525.9 & 690.5 & 445.9 & 449.4 & 949.4 & $9,283.1$ \\
\hline Wisconsin & $6,282.8$ & $2,320.3$ & $2,921.3$ & $2,063.8$ & $2,064.1$ & $1,615.3$ & $2,095.9$ & $1,295.7$ & $1,444.7$ & $2,949.7$ & $25,053.4$ \\
\hline Wyoming & 640.2 & 212.5 & 336.8 & 191.9 & 320.1 & 132.0 & 185.1 & 118.4 & 122.1 & 255.8 & $2,514.8$ \\
\hline
\end{tabular}


Representative State-Local Expenditures by Function, 1999, per capita, Adjusted for Input Cost Differences

\begin{tabular}{|c|c|c|c|c|c|c|c|c|c|c|c|c|}
\hline & Population & $\begin{array}{c}\text { Primary and } \\
\text { Secondary } \\
\text { Education }\end{array}$ & $\begin{array}{c}\text { Higher } \\
\text { Education }\end{array}$ & Public Welfare & $\begin{array}{c}\text { Health and } \\
\text { Hospitals }\end{array}$ & Highways & $\begin{array}{l}\text { Police and } \\
\text { Corrections }\end{array}$ & $\begin{array}{l}\text { Environment and } \\
\text { Housing }\end{array}$ & $\begin{array}{c}\text { Interest on } \\
\text { General debt }\end{array}$ & $\begin{array}{r}\text { Governmental } \\
\text { Administration }\end{array}$ & All Other & Total \\
\hline Alabama & $4,369,862$ & $1,129.2$ & 437.3 & 940.4 & 507.7 & 443.1 & 382.4 & 391.2 & 246.8 & 262.8 & 544.9 & $5,285.9$ \\
\hline Alaska & 619,500 & $1,758.4$ & 528.0 & 550.8 & 371.3 & 299.9 & 485.8 & 415.5 & 246.8 & 300.5 & 596.7 & $5,553.7$ \\
\hline Arizona & $4,778,332$ & $1,325.3$ & 428.0 & 911.8 & 435.2 & 322.5 & 392.1 & 395.5 & 246.8 & 269.5 & 554.0 & $5,280.6$ \\
\hline Arkansas & $2,551,373$ & $1,167.9$ & 400.4 & 926.5 & 519.4 & 457.4 & 310.6 & 381.3 & 246.8 & 247.4 & 523.6 & $5,181.3$ \\
\hline California & $33,145,121$ & $1,373.2$ & 500.6 & 945.8 & 427.0 & 287.9 & 407.2 & 415.1 & 246.8 & 299.8 & 595.8 & $5,499.2$ \\
\hline Colorado & $4,056,133$ & $1,305.0$ & 448.5 & 557.9 & 388.9 & 372.4 & 339.3 & 402.5 & 246.8 & 280.4 & 569.0 & $4,910.7$ \\
\hline Connecticut & $3,282,031$ & $1,315.5$ & 438.8 & 541.1 & 422.7 & 300.0 & 328.6 & 423.2 & 246.8 & 312.4 & 613.0 & $4,942.0$ \\
\hline Delaware & 753,538 & $1,102.4$ & 457.8 & 663.5 & 440.1 & 360.3 & 315.7 & 408.2 & 246.8 & 289.1 & 581.0 & $4,865.0$ \\
\hline D.C. & 519,000 & 782.1 & 488.9 & $1,207.6$ & 504.7 & 211.6 & $1,341.9$ & 417.9 & 246.8 & 304.2 & 601.8 & $6,107.5$ \\
\hline Florida & $15,111,244$ & $1,050.6$ & 374.2 & 812.5 & 438.2 & 291.9 & 314.5 & 390.0 & 246.8 & 260.9 & 542.2 & $4,721.8$ \\
\hline Georgia & $7,788,240$ & $1,280.4$ & 463.4 & 845.6 & 463.9 & 421.3 & 400.8 & 401.3 & 246.8 & 278.4 & 566.2 & $5,367.9$ \\
\hline Hawaii & $1,185,497$ & $1,048.2$ & 446.7 & 696.0 & 353.5 & 207.9 & 314.8 & 397.3 & 246.8 & 272.2 & 557.8 & $4,541.2$ \\
\hline Idaho & $1,251,700$ & $1,360.5$ & 457.6 & 854.8 & 406.0 & 450.4 & 275.1 & 387.0 & 246.8 & 256.2 & 535.7 & $5,230.2$ \\
\hline Illinois & $12,128,370$ & $1,252.9$ & 462.1 & 644.9 & 407.5 & 292.2 & 422.1 & 409.5 & 246.8 & 291.1 & 583.7 & $5,012.7$ \\
\hline Indiana & $5,942,901$ & $1,220.6$ & 450.1 & 512.5 & 421.4 & 399.1 & 378.6 & 401.2 & 246.8 & 278.3 & 566.1 & $4,874.6$ \\
\hline lowa & $2,869,413$ & $1,138.3$ & 414.9 & 525.8 & 386.8 & 434.1 & 248.0 & 387.5 & 246.8 & 257.1 & 536.9 & $4,576.2$ \\
\hline Kansas & $2,654,052$ & $1,227.6$ & 431.6 & 691.7 & 394.3 & 486.7 & 343.7 & 389.6 & 246.8 & 260.3 & 541.3 & $5,013.6$ \\
\hline Kentucky & $3,960,825$ & $1,107.7$ & 441.6 & 813.6 & 540.6 & 410.5 & 335.6 & 391.6 & 246.8 & 263.4 & 545.7 & $5,097.1$ \\
\hline Louisiana & $4,372,035$ & $1,171.8$ & 447.8 & $1,209.7$ & 479.6 & 315.1 & 435.0 & 387.3 & 246.8 & 256.7 & 536.4 & $5,486.2$ \\
\hline Maine & $1,253,040$ & $1,095.8$ & 384.7 & 663.6 & 468.7 & 378.8 & 243.5 & 384.6 & 246.8 & 252.5 & 530.7 & $4,649.6$ \\
\hline Maryland & $5,171,634$ & $1,222.7$ & 455.8 & 467.1 & 379.1 & 305.9 & 460.5 & 417.1 & 246.8 & 303.0 & 600.0 & $4,858.0$ \\
\hline Massachusetts & $6,175,169$ & $1,187.3$ & 440.7 & 659.3 & 459.7 & 268.9 & 285.4 & 412.5 & 246.8 & 295.8 & 590.1 & $4,846.4$ \\
\hline Michigan & $9,863,775$ & $1,362.9$ & 471.5 & 666.4 & 442.2 & 335.9 & 417.9 & 413.6 & 246.8 & 297.6 & 592.6 & $5,247.4$ \\
\hline Minnesota & $4,775,508$ & $1,314.7$ & 447.9 & 565.0 & 370.6 & 420.6 & 302.9 & 404.0 & 246.8 & 282.6 & 572.1 & $4,927.1$ \\
\hline Mississippi & $2,768,619$ & $1,184.9$ & 429.7 & $1,064.5$ & 524.1 & 444.0 & 357.9 & 380.6 & 246.8 & 246.3 & 522.2 & $5,400.9$ \\
\hline Missouri & $5,468,338$ & $1,155.5$ & 415.6 & 679.5 & 433.5 & 426.6 & 348.2 & 390.5 & 246.8 & 261.7 & 543.3 & $4,901.2$ \\
\hline Montana & 882,779 & $1,171.0$ & 383.6 & $1,009.1$ & 428.6 & 596.7 & 245.2 & 374.5 & 246.8 & 236.9 & 509.2 & $5,201.6$ \\
\hline Nebraska & $1,666,028$ & $1,137.6$ & 417.2 & 733.2 & 375.6 & 511.0 & 286.2 & 384.9 & 246.8 & 252.9 & 531.2 & $4,876.5$ \\
\hline Nevada & $1,809,253$ & $1,325.1$ & 409.8 & 697.2 & 413.3 & 353.6 & 413.0 & 399.4 & 246.8 & 275.5 & 562.2 & $5,095.8$ \\
\hline New Hampshire & $1,201,134$ & $1,231.0$ & 407.0 & 563.5 & 409.8 & 332.4 & 252.3 & 400.8 & 246.8 & 277.6 & 565.2 & $4,686.3$ \\
\hline New Jersey & $8,143,412$ & $1,255.6$ & 460.5 & 535.6 & 420.5 & 261.1 & 346.5 & 425.5 & 246.8 & 316.0 & 618.0 & $4,886.1$ \\
\hline New Mexico & $1,739,844$ & $1,336.2$ & 409.8 & $1,294.9$ & 448.9 & 487.1 & 397.2 & 384.0 & 246.8 & 251.6 & 529.3 & $5,785.7$ \\
\hline New York & $18,196,601$ & $1,161.5$ & 449.3 & 993.4 & 469.9 & 227.8 & 354.8 & 409.9 & 246.8 & 291.7 & 584.6 & $5,189.5$ \\
\hline North Carolina & $7,650,789$ & $1,179.2$ & 416.2 & 877.1 & 486.1 & 371.2 & 361.0 & 391.6 & 246.8 & 263.4 & 545.6 & $5,138.1$ \\
\hline North Dakota & 633,666 & $1,145.6$ & 410.3 & 884.6 & 381.4 & 822.8 & 236.9 & 375.7 & 246.8 & 238.7 & 511.6 & $5,254.4$ \\
\hline Ohio & $11,256,654$ & $1,224.7$ & 448.7 & 744.6 & 433.9 & 310.8 & 317.2 & 403.8 & 246.8 & 282.2 & 571.5 & $4,984.3$ \\
\hline Oklahoma & $3,358,044$ & $1,205.4$ & 409.3 & 844.8 & 426.0 & 474.8 & 338.4 & 381.7 & 246.8 & 247.9 & 524.4 & $5,099.5$ \\
\hline Oregon & $3,316,154$ & $1,224.0$ & 429.1 & 881.6 & 419.3 & 376.1 & 285.9 & 397.9 & 246.8 & 273.1 & 559.0 & $5,092.8$ \\
\hline Pennsylvania & $11,994,016$ & $1,113.9$ & 422.9 & 662.0 & 424.1 & 285.2 & 339.1 & 405.3 & 246.8 & 284.6 & 574.7 & $4,758.4$ \\
\hline Rhode Island & 990,819 & $1,135.0$ & 420.5 & 686.4 & 459.0 & 264.0 & 305.2 & 402.9 & 246.8 & 281.0 & 569.8 & $4,770.6$ \\
\hline South Carolina & $3,885,736$ & $1,132.4$ & 433.7 & 805.4 & 477.1 & 380.9 & 352.2 & 388.9 & 246.8 & 259.2 & 539.9 & $5,016.4$ \\
\hline South Dakota & 733,133 & $1,157.0$ & 398.5 & 582.7 & 367.0 & 727.8 & 247.1 & 374.1 & 246.8 & 236.2 & 508.2 & $4,845.2$ \\
\hline Tennessee & $5,483,535$ & $1,109.0$ & 421.3 & 807.1 & 497.7 & 392.5 & 361.2 & 391.4 & 246.8 & 263.1 & 545.2 & $5,035.4$ \\
\hline Texas & $20,044,141$ & $1,389.5$ & 461.1 & 958.0 & 410.1 & 358.7 & 368.1 & 397.5 & 246.8 & 272.5 & 558.2 & $5,420.5$ \\
\hline Utah & $2,129,836$ & $1,644.7$ & 581.8 & 468.0 & 350.5 & 375.4 & 342.1 & 402.3 & 246.8 & 280.0 & 568.5 & $5,260.1$ \\
\hline Vermont & 593,740 & $1,075.3$ & 396.6 & 620.6 & 426.4 & 412.4 & 262.9 & 387.0 & 246.8 & 256.3 & 535.8 & $4,619.9$ \\
\hline Virginia & $6,872,912$ & $1,202.1$ & 474.6 & 540.5 & 422.6 & 353.7 & 375.6 & 406.8 & 246.8 & 287.0 & 578.1 & $4,887.9$ \\
\hline Washington & $5,756,361$ & $1,319.8$ & 463.1 & 591.9 & 402.2 & 321.6 & 314.5 & 406.7 & 246.8 & 286.8 & 577.8 & $4,931.2$ \\
\hline West Virginia & $1,806,928$ & $1,060.2$ & 406.4 & $1,059.6$ & 552.9 & 364.4 & 291.0 & 382.1 & 246.8 & 248.7 & 525.4 & $5,137.5$ \\
\hline Wisconsin & $5,250,446$ & $1,196.6$ & 441.9 & 556.4 & 393.1 & 393.1 & 307.6 & 399.2 & 246.8 & 275.2 & 561.8 & $4,771.7$ \\
\hline Wyoming & 479,602 & $1,335.0$ & 443.1 & 702.2 & 400.1 & 667.4 & 275.2 & 385.9 & 246.8 & 254.5 & 533.4 & $5,243.5$ \\
\hline $\begin{array}{l}\text { Totals } \\
\end{array}$ & $\overline{272,690,813}$ & $\overline{~ 1,239.3}$ & $\bar{~} 447.9$ & 784.9 & $\bar{~} 438.7$ & 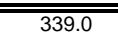 & 360.7 & 402.4 & 246.8 & $\overline{280.2}$ & 568.7 & $5,108.6$ \\
\hline
\end{tabular}




\begin{tabular}{|c|c|c|c|c|c|c|c|c|c|c|c|c|}
\hline & Population & $\begin{array}{l}\text { Primary and } \\
\text { Secondary } \\
\text { Education }\end{array}$ & $\begin{array}{c}\text { Higher } \\
\text { Education }\end{array}$ & $\begin{array}{c}\text { Public } \\
\text { Welfare }\end{array}$ & $\begin{array}{c}\text { Health and } \\
\text { Hospitals }\end{array}$ & Highways & $\begin{array}{l}\text { Police and } \\
\text { Corrections }\end{array}$ & $\begin{array}{l}\text { Environment } \\
\text { and Housing }\end{array}$ & $\begin{array}{c}\text { Interest on General } \\
\text { debt }\end{array}$ & $\begin{array}{r}\text { Governmental } \\
\text { Administration }\end{array}$ & All Other & Total \\
\hline Alabama & $4,369,862$ & $1,129.2$ & 437.3 & 940.4 & 464.2 & 443.1 & 382.4 & 391.2 & 246.8 & 262.8 & 544.9 & $5,242.4$ \\
\hline Alaska & 619,500 & $1,758.4$ & 528.0 & 550.8 & 437.3 & 299.9 & 485.8 & 415.5 & 246.8 & 300.5 & 596.7 & $5,619.7$ \\
\hline Arizona & $4,778,332$ & $1,325.3$ & 428.0 & 911.8 & 403.9 & 322.5 & 392.1 & 395.5 & 246.8 & 269.5 & 554.0 & $5,249.3$ \\
\hline Arkansas & $2,551,373$ & $1,167.9$ & 400.4 & 926.5 & 462.9 & 457.4 & 310.6 & 381.3 & 246.8 & 247.4 & 523.6 & $5,124.8$ \\
\hline California & $33,145,121$ & $1,373.2$ & 500.6 & 945.8 & 410.6 & 287.9 & 407.2 & 415.1 & 246.8 & 299.8 & 595.8 & $5,482.8$ \\
\hline Colorado & $4,056,133$ & $1,305.0$ & 448.5 & 557.9 & 457.8 & 372.4 & 339.3 & 402.5 & 246.8 & 280.4 & 569.0 & $4,979.5$ \\
\hline Connecticut & $3,282,031$ & $1,315.5$ & 438.8 & 541.1 & 512.5 & 300.0 & 328.6 & 423.2 & 246.8 & 312.4 & 613.0 & $5,031.9$ \\
\hline Delaware & 753,538 & $1,102.4$ & 457.8 & 663.5 & 468.6 & 360.3 & 315.7 & 408.2 & 246.8 & 289.1 & 581.0 & $4,893.5$ \\
\hline D.C. & 519,000 & 782.1 & 488.9 & $1,207.6$ & 416.1 & 211.6 & $1,341.9$ & 417.9 & 246.8 & 304.2 & 601.8 & $6,018.9$ \\
\hline Florida & $15,111,244$ & $1,050.6$ & 374.2 & 812.5 & 418.9 & 291.9 & 314.5 & 390.0 & 246.8 & 260.9 & 542.2 & $4,702.5$ \\
\hline Georgia & $7,788,240$ & $1,280.4$ & 463.4 & 845.6 & 441.4 & 421.3 & 400.8 & 401.3 & 246.8 & 278.4 & 566.2 & $5,345.4$ \\
\hline Hawaii & $1,185,497$ & $1,048.2$ & 446.7 & 696.0 & 389.8 & 207.9 & 314.8 & 397.3 & 246.8 & 272.2 & 557.8 & $\begin{array}{l}4,577.5 \\
4,5\end{array}$ \\
\hline Idaho & $1,251,700$ & $1,360.5$ & 457.6 & 854.8 & 379.8 & 450.4 & 275.1 & 387.0 & 246.8 & 256.2 & 535.7 & $5,203.9$ \\
\hline Illinois & $12,128,370$ & $1,252.9$ & 462.1 & 644.9 & 441.5 & 292.2 & 422.1 & 409.5 & 246.8 & 291.1 & 583.7 & $5,046.8$ \\
\hline Indiana & $5,942,901$ & $1,220.6$ & 450.1 & 512.5 & 461.0 & 399.1 & 378.6 & 401.2 & 246.8 & 278.3 & 566.1 & $4,914.2$ \\
\hline lowa & $2,869,413$ & $1,138.3$ & 414.9 & 525.8 & 405.4 & 434.1 & 248.0 & 387.5 & 246.8 & 257.1 & 536.9 & $4,594.8$ \\
\hline Kansas & $2,654,052$ & $1,227.6$ & 431.6 & 691.7 & 394.9 & 486.7 & 343.7 & 389.6 & 246.8 & 260.3 & 541.3 & $5,014.3$ \\
\hline Kentucky & $3,960,825$ & $1,107.7$ & 441.6 & 813.6 & 506.4 & 410.5 & 335.6 & 391.6 & 246.8 & 263.4 & 545.7 & $5,062.8$ \\
\hline Louisiana & $4,372,035$ & $1,171.8$ & 447.8 & $1,209.7$ & 392.1 & 315.1 & 435.0 & 387.3 & 246.8 & 256.7 & 536.4 & $5,398.8$ \\
\hline Maine & $1,253,040$ & $1,095.8$ & 384.7 & 663.6 & 476.9 & 378.8 & 243.5 & 384.6 & 246.8 & 252.5 & 530.7 & $4,657.8$ \\
\hline Maryland & $5,171,634$ & $1,222.7$ & 455.8 & 467.1 & 485.1 & 305.9 & 460.5 & 417.1 & 246.8 & 303.0 & 600.0 & $4,964.0$ \\
\hline Massachusetts & $6,175,169$ & $1,187.3$ & 440.7 & 659.3 & 494.0 & 268.9 & 285.4 & 412.5 & 246.8 & 295.8 & 590.1 & $4,880.7$ \\
\hline Michigan & $9,863,775$ & $1,362.9$ & 471.5 & 666.4 & 484.3 & 335.9 & 417.9 & 413.6 & 246.8 & 297.6 & 592.6 & $5,289.6$ \\
\hline Minnesota & $4,775,508$ & $1,314.7$ & 447.9 & 565.0 & 456.8 & 420.6 & 302.9 & 404.0 & 246.8 & 282.6 & 572.1 & $5,013.3$ \\
\hline Mississippi & $2,768,619$ & $1,184.9$ & 429.7 & $1,064.5$ & 458.4 & 444.0 & 357.9 & 380.6 & 246.8 & 246.3 & 522.2 & $5,335.3$ \\
\hline Missouri & $5,468,338$ & $1,155.5$ & 415.6 & 679.5 & 455.7 & 426.6 & 348.2 & 390.5 & 246.8 & 261.7 & 543.3 & $4,923.4$ \\
\hline Montana & 882,779 & $1,171.0$ & 383.6 & $1,009.1$ & 369.4 & 596.7 & 245.2 & 374.5 & 246.8 & 236.9 & 509.2 & $5,142.4$ \\
\hline Nebraska & $1,666,028$ & $1,137.6$ & 417.2 & 733.2 & 385.9 & 511.0 & 286.2 & 384.9 & 246.8 & 252.9 & 531.2 & $4,886.8$ \\
\hline Nevada & $1,809,253$ & $1,325.1$ & 409.8 & 697.2 & 426.5 & 353.6 & 413.0 & 399.4 & 246.8 & 275.5 & 562.2 & $5,109.0$ \\
\hline New Hampshire & $1,201,134$ & $1,231.0$ & 407.0 & 563.5 & 474.0 & 332.4 & 252.3 & 400.8 & 246.8 & 277.6 & 565.2 & $4,750.5$ \\
\hline New Jersey & $8,143,412$ & $1,255.6$ & 460.5 & 535.6 & 503.4 & 261.1 & 346.5 & 425.5 & 246.8 & 316.0 & 618.0 & $4,969.0$ \\
\hline New Mexico & $1,739,844$ & $1,336.2$ & 409.8 & $1,294.9$ & 370.3 & 487.1 & 397.2 & 384.0 & 246.8 & 251.6 & 529.3 & $5,707.1$ \\
\hline New York & $18,196,601$ & $1,161.5$ & 449.3 & 993.4 & 448.4 & 227.8 & 354.8 & 409.9 & 246.8 & 291.7 & 584.6 & $5,168.0$ \\
\hline North Carolina & $7,650,789$ & $1,179.2$ & 416.2 & 877.1 & 458.9 & 371.2 & 361.0 & 391.6 & 246.8 & 263.4 & 545.6 & $5,111.0$ \\
\hline North Dakota & 633,666 & $1,145.6$ & 410.3 & 884.6 & 343.6 & 822.8 & 236.9 & 375.7 & 246.8 & 238.7 & 511.6 & $5,216.6$ \\
\hline Ohio & $11,256,654$ & $1,224.7$ & 448.7 & 744.6 & 444.3 & 310.8 & 317.2 & 403.8 & 246.8 & 282.2 & 571.5 & $4,994.7$ \\
\hline Oklahoma & $3,358,044$ & $1,205.4$ & 409.3 & 844.8 & 391.4 & 474.8 & 338.4 & 381.7 & 246.8 & 247.9 & 524.4 & $5,064.8$ \\
\hline Oregon & $3,316,154$ & $1,224.0$ & 429.1 & 881.6 & 412.5 & 376.1 & 285.9 & 397.9 & 246.8 & 273.1 & 559.0 & $5,086.0$ \\
\hline Pennsylvania & $11,994,016$ & $1,113.9$ & 422.9 & 662.0 & 451.2 & 285.2 & 339.1 & 405.3 & 246.8 & 284.6 & 574.7 & $4,785.5$ \\
\hline Rhode Island & 990,819 & $1,135.0$ & 420.5 & 686.4 & 502.6 & 264.0 & 305.2 & 402.9 & 246.8 & 281.0 & 569.8 & $4,814.2$ \\
\hline South Carolina & $3,885,736$ & $1,132.4$ & 433.7 & 805.4 & 466.1 & 380.9 & 352.2 & 388.9 & 246.8 & 259.2 & 539.9 & $5,005.5$ \\
\hline South Dakota & 733,133 & $1,157.0$ & 398.5 & 582.7 & 382.7 & 727.8 & 247.1 & 374.1 & 246.8 & 236.2 & 508.2 & $4,860.9$ \\
\hline Tennessee & $5,483,535$ & $1,109.0$ & 421.3 & 807.1 & 460.2 & 392.5 & 361.2 & 391.4 & 246.8 & 263.1 & 545.2 & $4,997.9$ \\
\hline Texas & $20,044,141$ & $1,389.5$ & 461.1 & 958.0 & 369.8 & 358.7 & 368.1 & 397.5 & 246.8 & 272.5 & 558.2 & $5,380.2$ \\
\hline Utah & $2,129,836$ & $1,644.7$ & 581.8 & 468.0 & 403.2 & 375.4 & 342.1 & 402.3 & 246.8 & 280.0 & 568.5 & $5,312.8$ \\
\hline Vermont & 593,740 & $1,075.3$ & 396.6 & 620.6 & 432.0 & 412.4 & 262.9 & 387.0 & 246.8 & 256.3 & 535.8 & $4,625.6$ \\
\hline Virginia & $6,872,912$ & $1,202.1$ & 474.6 & 540.5 & 475.8 & 353.7 & 375.6 & 406.8 & 246.8 & 287.0 & 578.1 & $4,941.2$ \\
\hline Washington & $5,756,361$ & $1,319.8$ & 463.1 & 591.9 & 447.6 & 321.6 & 314.5 & 406.7 & 246.8 & 286.8 & 577.8 & $4,976.6$ \\
\hline West Virginia & $1,806,928$ & $1,060.2$ & 406.4 & $1,059.6$ & 478.4 & 364.4 & 291.0 & 382.1 & 246.8 & 248.7 & 525.4 & $5,063.0$ \\
\hline Wisconsin & $5,250,446$ & $1,196.6$ & 441.9 & 556.4 & 439.5 & 393.1 & 307.6 & 399.2 & 246.8 & 275.2 & 561.8 & $4,818.1$ \\
\hline Wyoming & 479,602 & $1,335.0$ & 443.1 & 702.2 & 391.0 & 667.4 & 275.2 & 385.9 & 246.8 & 254.5 & 533.4 & $5,234.4$ \\
\hline $\begin{array}{ll}\text { Totals } \\
\end{array}$ & $\overline{27272,690,813}$ & $\bar{~} 1,239.3$ & $\overline{4447.9}$ & $\overline{7784.9}$ & $\bar{~} 438.7$ & $\bar{~} 339.0$ & 360.7 & 402.4 & 246.8 & 280.2 & "568.7 & $5,108.6$ \\
\hline
\end{tabular}


Ratio of Actual Direct General Expenditures of State and Local Governments to Representative State-Local Expenditures Adjusted for Input Cost Differences, by Function, 1999

\begin{tabular}{|c|c|c|c|c|c|c|c|c|c|c|c|}
\hline & $\begin{array}{l}\text { Primary and } \\
\text { Secondary } \\
\text { Education }\end{array}$ & $\begin{array}{c}\text { Higher } \\
\text { Education }\end{array}$ & $\begin{array}{c}\text { Public } \\
\text { Welfare }\end{array}$ & $\begin{array}{c}\text { Health and } \\
\text { Hospitals }\end{array}$ & Highways & $\begin{array}{l}\text { Police and } \\
\text { Corrections }\end{array}$ & $\begin{array}{c}\text { Environment and } \\
\text { Housing }\end{array}$ & $\begin{array}{c}\text { Interest on } \\
\text { General debt }\end{array}$ & $\begin{array}{c}\text { Governmental } \\
\text { Administration }\end{array}$ & All Other & Total \\
\hline Alabama & 92.4 & 115.0 & 78.6 & 159.4 & 64.5 & 63.1 & 83.0 & 79.5 & 55.8 & 70.4 & 87.8 \\
\hline Alaska & 123.8 & 104.3 & 202.0 & 92.4 & 402.7 & 110.4 & 231.0 & 315.9 & 212.3 & 527.3 & 204.8 \\
\hline Arizona & 77.0 & 110.8 & 43.3 & 51.2 & 112.8 & 100.6 & 93.8 & 120.7 & 70.5 & 96.8 & 80.9 \\
\hline Arkansas & 85.6 & 110.0 & 80.6 & 91.4 & 73.7 & 78.0 & 68.3 & 80.8 & 54.9 & 75.3 & 81.5 \\
\hline California & 85.4 & 93.6 & 86.6 & 123.2 & 77.4 & 108.6 & 124.3 & 150.3 & 77.0 & 119.0 & 99.5 \\
\hline Colorado & 90.1 & 125.4 & 113.9 & 53.8 & 116.8 & 113.8 & 97.5 & 107.4 & 96.8 & 101.6 & 99.4 \\
\hline Connecticut & 111.5 & 77.3 & 165.0 & 91.9 & 112.4 & 109.7 & 91.1 & 157.4 & 125.9 & 157.3 & 119.2 \\
\hline Delaware & 112.1 & 156.2 & 100.6 & 73.7 & 142.9 & 148.7 & 125.4 & 199.4 & 138.8 & 102.6 & 121.6 \\
\hline D.C. & 171.1 & 32.5 & 198.9 & 236.0 & 92.0 & 87.0 & 175.7 & 303.0 & 160.8 & 117.4 & 148.2 \\
\hline Florida & 99.3 & 84.3 & 72.1 & 104.3 & 114.6 & 141.0 & 130.6 & 115.0 & 99.7 & 97.3 & 100.8 \\
\hline Georgia & 98.2 & 86.9 & 74.7 & 106.7 & 75.5 & 79.1 & 84.4 & 103.3 & 50.3 & 82.7 & 86.1 \\
\hline Hawaii & 86.1 & 129.6 & 115.9 & 118.6 & 163.6 & 95.2 & 133.1 & 150.3 & 184.6 & 182.6 & 126.9 \\
\hline Idaho & 80.9 & 101.7 & 67.4 & 114.7 & 93.0 & 115.9 & 96.1 & 122.7 & 53.8 & 66.3 & 86.2 \\
\hline Illinois & 101.4 & 82.5 & 110.7 & 78.0 & 107.6 & 84.3 & 105.5 & 105.3 & 99.0 & 96.6 & 97.6 \\
\hline Indiana & 95.9 & 122.7 & 127.8 & 90.6 & 77.0 & 63.0 & 78.1 & $\begin{array}{l}89.4 \\
\end{array}$ & 54.4 & 80.5 & 91.2 \\
\hline lowa & 105.7 & 162.9 & 137.7 & 139.2 & 129.0 & 95.4 & 88.1 & 99.1 & 42.5 & 85.4 & 111.4 \\
\hline Kansas & 90.9 & 142.9 & 70.6 & 95.9 & 103.7 & 81.5 & 70.7 & 107.4 & 63.2 & 84.4 & 90.7 \\
\hline Kentucky & 85.9 & 105.5 & 111.5 & 54.6 & 97.2 & 71.0 & 71.1 & 88.5 & 131.8 & 87.5 & 90.1 \\
\hline Louisiana & 90.0 & 92.2 & 56.4 & 179.5 & 112.4 & 78.6 & 97.2 & 103.6 & 83.1 & 81.1 & 89.5 \\
\hline Maine & 111.3 & 92.6 & 171.1 & 65.8 & 107.9 & 86.3 & 96.6 & 109.7 & 83.1 & 113.4 & 109.5 \\
\hline Maryland & 98.9 & 106.5 & 152.1 & 48.1 & 93.0 & 80.8 & 117.0 & 114.8 & 71.6 & 87.7 & 96.9 \\
\hline Massachusetts & 108.7 & 71.5 & 143.5 & 71.7 & 163.8 & 124.7 & 121.4 & 108.1 & 125.0 & 140.4 & 116.1 \\
\hline Michigan & 107.4 & 134.0 & 98.2 & 88.6 & 91.8 & 85.8 & 80.7 & 100.6 & 76.0 & 79.6 & 96.9 \\
\hline Minnesota & 108.9 & 114.3 & 203.9 & 79.1 & 110.6 & 94.7 & 127.7 & 134.6 & 108.9 & 93.1 & 117.6 \\
\hline Mississippi & 83.7 & 117.9 & 69.2 & 151.9 & 92.9 & 68.5 & 63.1 & 89.2 & 66.0 & 88.3 & 87.6 \\
\hline Missouri & 95.2 & 94.6 & 102.5 & 84.3 & 82.4 & 78.8 & 65.2 & 95.1 & 65.3 & 93.2 & 88.7 \\
\hline Montana & 98.3 & 140.2 & 57.4 & 91.6 & 91.3 & 111.9 & 111.4 & 134.2 & 108.2 & 84.8 & 94.6 \\
\hline Nebraska & 103.9 & 151.4 & 92.5 & 84.4 & 94.2 & 82.4 & 90.0 & 87.1 & 50.3 & 77.0 & 94.8 \\
\hline Nevada & 91.6 & 92.1 & 67.6 & 87.4 & 149.9 & 116.3 & 109.9 & 175.1 & 125.1 & 85.0 & 100.6 \\
\hline New Hampshire & 92.7 & 85.6 & 145.7 & 27.4 & 114.7 & 93.5 & 70.3 & 102.2 & 119.8 & 90.6 & 93.4 \\
\hline New Jersey & 130.8 & 82.4 & 130.7 & 50.5 & 112.4 & 120.4 & 99.9 & 127.6 & 89.3 & 118.7 & 109.5 \\
\hline New Mexico & 88.2 & 160.5 & 61.3 & 131.3 & 133.6 & 93.6 & 99.3 & 121.7 & 83.8 & 77.4 & 95.3 \\
\hline New York & 145.3 & 70.2 & 155.2 & 135.0 & 158.2 & 151.9 & 117.4 & 138.4 & 160.8 & 172.9 & 142.2 \\
\hline North Carolina & 92.1 & 129.5 & 84.8 & 150.9 & 88.5 & 85.4 & 92.5 & 78.3 & 55.7 & 74.3 & 94.0 \\
\hline North Dakota & 96.8 & 167.8 & 111.0 & 23.8 & 79.2 & 75.8 & 162.0 & 102.5 & 84.0 & 146.4 & 105.5 \\
\hline Ohio & 98.3 & 92.5 & 105.6 & 89.4 & 101.6 & 104.5 & 90.6 & 118.5 & 60.1 & 77.7 & 94.5 \\
\hline Oklahoma & 90.8 & 117.2 & 61.3 & 95.9 & 70.4 & 75.6 & 72.4 & 83.5 & 55.9 & 68.3 & 79.7 \\
\hline Oregon & 99.7 & 136.2 & 94.8 & 106.2 & 99.4 & 140.4 & 128.6 & 156.8 & 68.0 & 104.1 & 108.5 \\
\hline Pennsylvania & 119.6 & 92.0 & 142.5 & 66.7 & 110.6 & 104.1 & 87.2 & 99.4 & 119.0 & 92.8 & 106.7 \\
\hline Rhode Island & 113.3 & 88.5 & 153.3 & 48.6 & 114.0 & 108.6 & 96.0 & 125.0 & 114.2 & 127.6 & 110.7 \\
\hline South Carolina & 101.8 & 103.1 & 100.1 & 156.0 & 69.9 & 84.1 & 69.4 & 85.1 & 63.7 & 65.2 & 93.7 \\
\hline South Dakota & 95.3 & 99.0 & 104.4 & 54.0 & 88.6 & 91.2 & 97.8 & 104.3 & 89.7 & 80.6 & 91.1 \\
\hline Tennessee & 91.0 & 95.4 & 97.7 & 117.0 & 78.5 & 72.7 & 88.3 & 82.5 & 57.3 & 73.3 & 88.2 \\
\hline Texas & 91.2 & 97.1 & 58.6 & 110.6 & 81.4 & 92.6 & 70.3 & 78.2 & 74.4 & 65.4 & 81.0 \\
\hline Utah & 68.1 & 123.3 & 135.3 & 84.0 & 109.5 & 98.0 & 91.7 & 153.9 & 64.3 & 73.6 & 92.3 \\
\hline Vermont & 128.7 & 144.5 & 149.2 & 23.9 & 129.1 & 65.0 & 93.6 & 132.3 & 96.5 & 88.9 & 110.3 \\
\hline Virginia & 98.8 & 96.3 & 108.9 & 74.4 & 111.5 & 87.8 & 91.0 & 114.5 & 87.9 & 83.6 & 95.1 \\
\hline Washington & 96.6 & 118.8 & 135.3 & 125.0 & 118.7 & 108.9 & 133.2 & 104.2 & 82.2 & 116.6 & 112.9 \\
\hline West Virginia & 108.3 & 104.8 & 87.2 & 59.8 & 127.2 & 59.2 & 66.5 & 110.4 & 90.8 & 72.6 & 89.9 \\
\hline Wisconsin & 120.4 & 127.1 & 141.3 & $\begin{array}{l}69.7 \\
69.7\end{array}$ & 115.6 & 122.7 & 105.4 & $\begin{array}{l}107.9 \\
10.9\end{array}$ & $\begin{array}{l}88.8 \\
88.8\end{array}$ & 85.9 & 110.9 \\
\hline $\begin{array}{l}\text { Wyoming } \\
\text { wyon }\end{array}$ & $\begin{array}{l}113.4 \\
113.1\end{array}$ & $\begin{array}{l}138.5 \\
138.5\end{array}$ & $\begin{array}{l}74.4 \\
74.4\end{array}$ & $\begin{array}{l}239.0 \\
239.0\end{array}$ & $\begin{array}{l}124.1 \\
12.1\end{array}$ & 142.9 & $\begin{array}{l}134.6 \\
134.6\end{array}$ & $\begin{array}{l}175.9 \\
17.9\end{array}$ & $\begin{array}{l}0.0 \\
103.5\end{array}$ & $\begin{array}{l}03.5 \\
103.7\end{array}$ & 125.6 \\
\hline United States & 100.0 & 100.0 & 100.0 & 100.0 & 100.0 & 100.0 & 100.0 & & 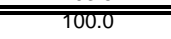 & 100.0 & \\
\hline
\end{tabular}


Workload Summary, 1999.

\begin{tabular}{|c|c|c|c|c|c|c|c|c|c|c|}
\hline & $\begin{array}{c}\text { Primary and Secondary } \\
\text { Education }\end{array}$ & $\begin{array}{c}\text { Higher } \\
\text { Education }\end{array}$ & Public Welfare & $\begin{array}{c}\text { Health and } \\
\text { Hospitals }\end{array}$ & Highways & $\begin{array}{l}\text { Police and } \\
\text { Corrections } \\
\end{array}$ & $\begin{array}{c}\text { Environment and } \\
\text { Housing }\end{array}$ & $\begin{array}{c}\text { Interest on General } \\
\text { debt }\end{array}$ & $\begin{array}{c}\text { Governmental } \\
\text { Administration } \\
\end{array}$ & All Other \\
\hline$\overline{\text { Alabama }}$ & $1.56 \%$ & $1.67 \%$ & $1.93 \%$ & $1.96 \%$ & $2.14 \%$ & $1.84 \%$ & $1.60 \%$ & $1.60 \%$ & $1.60 \%$ & $1.60 \%$ \\
\hline Alaska & $0.30 \%$ & $0.25 \%$ & $0.16 \%$ & $0.18 \%$ & $0.19 \%$ & $0.28 \%$ & $0.23 \%$ & $0.23 \%$ & $0.23 \%$ & $0.23 \%$ \\
\hline Arizona & $1.95 \%$ & $1.74 \%$ & $2.04 \%$ & $1.80 \%$ & $1.69 \%$ & $2.00 \%$ & $1.75 \%$ & $1.75 \%$ & $1.75 \%$ & $1.75 \%$ \\
\hline Arkansas & $1.00 \%$ & $0.95 \%$ & $1.12 \%$ & $1.23 \%$ & $1.32 \%$ & $0.94 \%$ & $0.94 \%$ & $0.94 \%$ & $0.94 \%$ & $0.94 \%$ \\
\hline California & $12.54 \%$ & $12.68 \%$ & $14.43 \%$ & $11.28 \%$ & $9.99 \%$ & $12.61 \%$ & $12.15 \%$ & $12.15 \%$ & $12.15 \%$ & $12.15 \%$ \\
\hline Colorado & $1.56 \%$ & $1.49 \%$ & $1.05 \%$ & $1.33 \%$ & $1.63 \%$ & $1.40 \%$ & $1.49 \%$ & $1.49 \%$ & $1.49 \%$ & $1.49 \%$ \\
\hline Connecticut & $1.14 \%$ & $1.06 \%$ & $0.81 \%$ & $1.07 \%$ & $1.01 \%$ & $0.96 \%$ & $1.20 \%$ & $1.20 \%$ & $1.20 \%$ & $1.20 \%$ \\
\hline Delaware & $0.24 \%$ & $0.27 \%$ & $0.23 \%$ & $0.27 \%$ & $0.29 \%$ & $0.23 \%$ & $0.28 \%$ & $0.28 \%$ & $0.28 \%$ & $0.28 \%$ \\
\hline D.C. & $0.11 \%$ & $0.19 \%$ & $0.29 \%$ & $0.21 \%$ & $0.11 \%$ & $0.64 \%$ & $0.19 \%$ & $0.19 \%$ & $0.19 \%$ & $0.19 \%$ \\
\hline Florida & $5.05 \%$ & $4.97 \%$ & $5.77 \%$ & $5.88 \%$ & $4.89 \%$ & $5.27 \%$ & $5.54 \%$ & $5.54 \%$ & $5.54 \%$ & $5.54 \%$ \\
\hline Georgia & $2.97 \%$ & $2.97 \%$ & $3.07 \%$ & $3.05 \%$ & $3.54 \%$ & $3.19 \%$ & $2.86 \%$ & $2.86 \%$ & $2.86 \%$ & $2.86 \%$ \\
\hline Hawaii & $0.38 \%$ & $0.45 \%$ & $0.39 \%$ & $0.36 \%$ & $0.27 \%$ & $0.39 \%$ & $0.43 \%$ & $0.43 \%$ & $0.43 \%$ & $0.43 \%$ \\
\hline Idaho & $0.55 \%$ & $0.51 \%$ & $0.50 \%$ & $0.46 \%$ & $0.63 \%$ & $0.39 \%$ & $0.46 \%$ & $0.46 \%$ & $0.46 \%$ & $0.46 \%$ \\
\hline Illinois & $4.32 \%$ & $4.41 \%$ & $3.62 \%$ & $4.03 \%$ & $3.76 \%$ & $4.96 \%$ & $4.45 \%$ & $4.45 \%$ & $4.45 \%$ & $4.45 \%$ \\
\hline Indiana & $2.16 \%$ & $2.20 \%$ & $1.42 \%$ & $2.12 \%$ & $2.56 \%$ & $2.30 \%$ & $2.18 \%$ & $2.18 \%$ & $2.18 \%$ & $2.18 \%$ \\
\hline lowa & $1.05 \%$ & $1.06 \%$ & $0.71 \%$ & $1.00 \%$ & $1.39 \%$ & $0.80 \%$ & $1.05 \%$ & $1.05 \%$ & $1.05 \%$ & $1.05 \%$ \\
\hline Kansas & $1.04 \%$ & $1.01 \%$ & $0.86 \%$ & $0.93 \%$ & $1.43 \%$ & $1.02 \%$ & $0.97 \%$ & $0.97 \%$ & $0.97 \%$ & $0.97 \%$ \\
\hline Kentucky & $1.38 \%$ & $1.52 \%$ & $1.51 \%$ & $1.89 \%$ & $1.79 \%$ & $1.46 \%$ & $1.45 \%$ & $1.45 \%$ & $1.45 \%$ & $1.45 \%$ \\
\hline Louisiana & $1.66 \%$ & $1.75 \%$ & $2.49 \%$ & $1.88 \%$ & $1.54 \%$ & $2.16 \%$ & $1.60 \%$ & $1.60 \%$ & $1.60 \%$ & $1.60 \%$ \\
\hline Maine & $0.45 \%$ & $0.44 \%$ & $0.39 \%$ & $0.53 \%$ & $0.53 \%$ & $0.35 \%$ & $0.46 \%$ & $0.46 \%$ & $0.46 \%$ & $0.46 \%$ \\
\hline Maryland & $1.72 \%$ & $1.78 \%$ & $1.11 \%$ & $1.55 \%$ & $1.65 \%$ & $2.20 \%$ & $1.90 \%$ & $1.90 \%$ & $1.90 \%$ & $1.90 \%$ \\
\hline Massachusetts & $2.05 \%$ & $2.11 \%$ & $1.88 \%$ & $2.29 \%$ & $1.75 \%$ & $1.67 \%$ & $2.26 \%$ & $2.26 \%$ & $2.26 \%$ & $2.26 \%$ \\
\hline Michigan & $3.73 \%$ & $3.58 \%$ & $3.03 \%$ & $3.50 \%$ & $3.48 \%$ & $3.89 \%$ & $3.62 \%$ & $3.62 \%$ & $3.62 \%$ & $3.62 \%$ \\
\hline Minnesota & $1.84 \%$ & $1.73 \%$ & $1.25 \%$ & $1.48 \%$ & $2.16 \%$ & $1.45 \%$ & $1.75 \%$ & $1.75 \%$ & $1.75 \%$ & $1.75 \%$ \\
\hline Mississippi & $1.11 \%$ & $1.11 \%$ & $1.40 \%$ & $1.35 \%$ & $1.39 \%$ & $1.18 \%$ & $1.02 \%$ & $1.02 \%$ & $1.02 \%$ & $1.02 \%$ \\
\hline Missouri & $2.00 \%$ & $1.99 \%$ & $1.74 \%$ & $2.10 \%$ & $2.58 \%$ & $2.11 \%$ & $2.01 \%$ & $2.01 \%$ & $2.01 \%$ & $2.01 \%$ \\
\hline Montana & $0.36 \%$ & $0.33 \%$ & $0.42 \%$ & $0.36 \%$ & $0.61 \%$ & $0.27 \%$ & $0.32 \%$ & $0.32 \%$ & $0.32 \%$ & $0.32 \%$ \\
\hline Nebraska & $0.62 \%$ & $0.63 \%$ & $0.58 \%$ & $0.57 \%$ & $0.95 \%$ & $0.55 \%$ & $0.61 \%$ & $0.61 \%$ & $0.61 \%$ & $0.61 \%$ \\
\hline Nevada & $0.72 \%$ & $0.62 \%$ & $0.59 \%$ & $0.64 \%$ & $0.69 \%$ & $0.77 \%$ & $0.66 \%$ & $0.66 \%$ & $0.66 \%$ & $0.66 \%$ \\
\hline New Hampshire & $0.44 \%$ & $0.40 \%$ & $0.32 \%$ & $0.42 \%$ & $0.43 \%$ & $0.31 \%$ & $0.44 \%$ & $0.44 \%$ & $0.44 \%$ & $0.44 \%$ \\
\hline New Jersey & $2.67 \%$ & $2.72 \%$ & $1.99 \%$ & $2.62 \%$ & $2.18 \%$ & $2.47 \%$ & $2.99 \%$ & $2.99 \%$ & $2.99 \%$ & $2.99 \%$ \\
\hline New Mexico & $0.77 \%$ & $0.65 \%$ & $1.06 \%$ & $0.71 \%$ & $0.95 \%$ & $0.80 \%$ & $0.64 \%$ & $0.64 \%$ & $0.64 \%$ & $0.64 \%$ \\
\hline New York & $5.99 \%$ & $6.42 \%$ & $8.35 \%$ & $6.96 \%$ & $4.39 \%$ & $6.24 \%$ & $6.67 \%$ & $6.67 \%$ & $6.67 \%$ & $6.67 \%$ \\
\hline North Carolina & $2.84 \%$ & $2.77 \%$ & $3.15 \%$ & $3.28 \%$ & $3.14 \%$ & $3.03 \%$ & $2.81 \%$ & $2.81 \%$ & $2.81 \%$ & $2.81 \%$ \\
\hline North Dakota & $0.25 \%$ & $0.25 \%$ & $0.27 \%$ & $0.23 \%$ & $0.60 \%$ & $0.19 \%$ & $0.23 \%$ & $0.23 \%$ & $0.23 \%$ & $0.23 \%$ \\
\hline Ohio & $4.04 \%$ & $4.10 \%$ & $3.89 \%$ & $4.08 \%$ & $3.76 \%$ & $3.59 \%$ & $4.13 \%$ & $4.13 \%$ & $4.13 \%$ & $4.13 \%$ \\
\hline Oklahoma & $1.36 \%$ & $1.27 \%$ & $1.34 \%$ & $1.32 \%$ & $1.80 \%$ & $1.35 \%$ & $1.23 \%$ & $1.23 \%$ & $1.23 \%$ & $1.23 \%$ \\
\hline Oregon & $1.23 \%$ & $1.19 \%$ & $1.36 \%$ & $1.19 \%$ & $1.36 \%$ & $0.99 \%$ & $1.22 \%$ & $1.22 \%$ & $1.22 \%$ & $1.22 \%$ \\
\hline Pennsylvania & $3.88 \%$ & $4.09 \%$ & $3.68 \%$ & $4.22 \%$ & $3.66 \%$ & $4.05 \%$ & $4.40 \%$ & $4.40 \%$ & $4.40 \%$ & $4.40 \%$ \\
\hline Rhode Island & $0.33 \%$ & $0.34 \%$ & $0.32 \%$ & $0.38 \%$ & $0.28 \%$ & $0.31 \%$ & $0.36 \%$ & $0.36 \%$ & $0.36 \%$ & $0.36 \%$ \\
\hline South Carolina & $1.41 \%$ & $1.49 \%$ & $1.47 \%$ & $1.65 \%$ & $1.64 \%$ & $1.53 \%$ & $1.42 \%$ & $1.42 \%$ & $1.42 \%$ & $1.42 \%$ \\
\hline South Dakota & $0.30 \%$ & $0.28 \%$ & $0.20 \%$ & $0.26 \%$ & $0.61 \%$ & $0.23 \%$ & $0.27 \%$ & $0.27 \%$ & $0.27 \%$ & $0.27 \%$ \\
\hline Tennessee & $1.92 \%$ & $2.01 \%$ & $2.08 \%$ & $2.41 \%$ & $2.38 \%$ & $2.18 \%$ & $2.01 \%$ & $2.01 \%$ & $2.01 \%$ & $2.01 \%$ \\
\hline Texas & $8.47 \%$ & $7.77 \%$ & $8.96 \%$ & $7.06 \%$ & $7.83 \%$ & $7.75 \%$ & $7.35 \%$ & $7.35 \%$ & $7.35 \%$ & $7.35 \%$ \\
\hline Utah & $1.04 \%$ & $1.01 \%$ & $0.46 \%$ & $0.63 \%$ & $0.86 \%$ & $0.74 \%$ & $0.78 \%$ & $0.78 \%$ & $0.78 \%$ & $0.78 \%$ \\
\hline Vermont & $0.21 \%$ & $0.21 \%$ & $0.17 \%$ & $0.23 \%$ & $0.27 \%$ & $0.18 \%$ & $0.22 \%$ & $0.22 \%$ & $0.22 \%$ & $0.22 \%$ \\
\hline Virginia & $2.38 \%$ & $2.60 \%$ & $1.72 \%$ & $2.40 \%$ & $2.59 \%$ & $2.54 \%$ & $2.52 \%$ & $2.52 \%$ & $2.52 \%$ & $2.52 \%$ \\
\hline Washington & $2.19 \%$ & $2.13 \%$ & $1.58 \%$ & $1.91 \%$ & $1.97 \%$ & $1.79 \%$ & $2.11 \%$ & $2.11 \%$ & $2.11 \%$ & $2.11 \%$ \\
\hline West Virginia & $0.64 \%$ & $0.68 \%$ & $0.91 \%$ & $0.92 \%$ & $0.74 \%$ & $0.62 \%$ & $0.66 \%$ & $0.66 \%$ & $0.66 \%$ & $0.66 \%$ \\
\hline Wisconsin & $1.89 \%$ & $1.93 \%$ & $1.36 \%$ & $1.76 \%$ & $2.24 \%$ & $1.68 \%$ & $1.93 \%$ & $1.93 \%$ & $1.93 \%$ & $1.93 \%$ \\
\hline Wyoming & $0.21 \%$ & $0.19 \%$ & $0.16 \%$ & $0.17 \%$ & $0.36 \%$ & $0.15 \%$ & $0.18 \%$ & $0.18 \%$ & $0.18 \%$ & $0.18 \%$ \\
\hline$\overline{~ T o t a l s ~}$ & $100.00 \%$ & $100.00 \%$ & $100.00 \%$ & 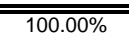 & 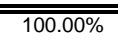 & 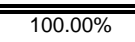 & 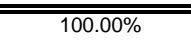 & 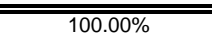 & $100.00 \%$ & 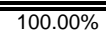 \\
\hline
\end{tabular}




\begin{tabular}{|c|c|c|c|c|c|c|c|c|c|c|c|}
\hline State & $\begin{array}{l}\text { Census } \\
\text { Region }\end{array}$ & $\begin{array}{c}1999 \\
\text { Elementary } \\
\text { Age Population } \\
(5-13) \\
\end{array}$ & $\begin{array}{c}1999 \\
\text { Secondary Age } \\
\text { Population (14- } \\
\text { 17) } \\
\end{array}$ & $\begin{array}{l}\text { Total Private } \\
\text { School } \\
\text { Enrollment (Fall } \\
\text { 1999) }\end{array}$ & $\begin{array}{c}\text { Elementary } \\
\text { Private School } \\
\text { Enrollment (calc) }\end{array}$ & $\begin{array}{c}\text { Secondary } \\
\text { Private School } \\
\text { Enrollment (calc) }\end{array}$ & $\begin{array}{l}\text { Percentage of } \\
\text { Persons under } \\
18 \text { Living in } \\
\text { Poverty }\end{array}$ & $\begin{array}{c}\text { Elementary Age } \\
\text { Population (5-13), } \\
\text { Net of Private } \\
\text { Enrollment }\end{array}$ & $\begin{array}{l}\text { Secondary Age } \\
\text { Population (14- } \\
\text { 17), Net of } \\
\text { Private } \\
\text { Enrollment } \\
\end{array}$ & $\begin{array}{c}\text { Education Cost } \\
\text { Index: }\left(1+25 \% \%^{*}(\% \text { in }\right. \\
\text { Poverty }))^{*} \\
(\text { Elementary * } 60 \%+ \\
\text { Secondary } 1)\end{array}$ & $\begin{array}{c}\text { Workload Measure: } \\
\text { Elementary and } \\
\text { Secondary Education: } \\
\text { Share of Education } \\
\text { Cost Index }\end{array}$ \\
\hline Alabama & South & 530,800 & 244,544 & 73,352 & 50,217 & 23,135 & $24 \%$ & 480,583 & 221,409 & 540,344 & $1.56 \%$ \\
\hline Alaska & West & 101,974 & 45,086 & 6,172 & 4,280 & 1,892 & $8 \%$ & 97,694 & 43,194 & 103,847 & $0.30 \%$ \\
\hline Arizona & West & 668,264 & 280,312 & 44,060 & 31,040 & 13,020 & $16 \%$ & 637,224 & 267,292 & 675,774 & $1.95 \%$ \\
\hline Arkansas & South & 328,109 & 154,466 & 26,424 & 17,966 & 8,458 & $19 \%$ & 310,143 & 146,008 & 347,453 & $1.00 \%$ \\
\hline California & West & $4,609,407$ & $1,814,758$ & 619,067 & 444,187 & 174,880 & $20 \%$ & $4,165,220$ & $1,639,878$ & $4,349,065$ & $12.54 \%$ \\
\hline Colorado & $\begin{array}{l}\text { West } \\
\end{array}$ & 531,393 & 245,902 & 52,142 & 35,647 & 16,495 & $12 \%$ & 495,746 & 229,407 & 542,133 & $1.56 \%$ \\
\hline Connecticut & Northeast & 432,344 & 177,751 & 70,058 & 49,647 & 20,411 & $9 \%$ & 382,697 & 157,340 & 395,471 & $1.14 \%$ \\
\hline Delaware & South & 92,296 & 39,989 & 22,779 & 15,893 & 6,886 & $18 \%$ & 76,403 & 33,103 & 82,418 & $0.24 \%$ \\
\hline D.C. & South & 50,686 & 17,304 & 16,690 & 12,442 & 4,248 & $25 \%$ & 38,244 & 13,056 & 38,208 & $0.11 \%$ \\
\hline Florida & South & $1,833,827$ & 783,677 & 290,872 & 203,785 & 87,087 & $18 \%$ & $1,630,042$ & 696,590 & $1,751,648$ & $5.05 \%$ \\
\hline Georgia & South & $1,029,126$ & 447,609 & 116,407 & 81,123 & 35,284 & $20 \%$ & 948,003 & 412,325 & $1,028,957$ & $2.97 \%$ \\
\hline Hawaii & West & 147,263 & 61,690 & 32,193 & 22,689 & 9,504 & $14 \%$ & 124,574 & 52,186 & 131,214 & $0.38 \%$ \\
\hline Idaho & West & 171,509 & 86,120 & 10,209 & 6,796 & 3,413 & $22 \%$ & 164,713 & 82,707 & 191,474 & $0.55 \%$ \\
\hline Illinois & Midwest & $1,611,853$ & 691,806 & 299,871 & 209,817 & 90,054 & $15 \%$ & $1,402,036$ & 601,752 & $1,497,085$ & $4.32 \%$ \\
\hline Indiana & Midwest & 763,919 & 351,397 & 105,533 & 72,283 & 33,250 & $9 \%$ & 691,636 & 318,147 & 748,708 & $2.16 \%$ \\
\hline Iowa & Midwest & 358,539 & 178,326 & 49,565 & 33,101 & 16,464 & $10 \%$ & 325,438 & 161,862 & 365,964 & $1.05 \%$ \\
\hline Kansas & Midwest & 346,666 & 167,958 & 43,113 & 29,042 & 14,071 & $19 \%$ & 317,624 & 153,887 & 360,393 & $1.04 \%$ \\
\hline Kentucky & South & 479,099 & 227,336 & 75,084 & 50,921 & 24,163 & $17 \%$ & 428,178 & 203,173 & 479,288 & $1.38 \%$ \\
\hline Louisiana & South & 589,547 & 286,304 & 138,135 & 92,981 & 45,154 & $27 \%$ & 496,566 & 241,150 & 574,939 & $1.66 \%$ \\
\hline Maine & Northeast & 149,198 & 74,023 & 18,287 & 12,223 & 6,064 & $18 \%$ & 136,975 & 67,959 & 156,713 & $0.45 \%$ \\
\hline Maryland & South & 677,286 & 285,288 & 144,131 & 101,413 & 42,718 & $7 \%$ & 575,873 & 242,570 & 597,798 & $1.72 \%$ \\
\hline Massachusetts & Northeast & 759,932 & 316,382 & 132,154 & 93,307 & 38,847 & $20 \%$ & 666,625 & 277,535 & 710,539 & $2.05 \%$ \\
\hline Michigan & Midwest & $1,313,980$ & 592,324 & 179,579 & 123,780 & 55,799 & $14 \%$ & $1,190,200$ & 536,525 & $1,294,730$ & $3.73 \%$ \\
\hline Minnesota & Midwest & 642,933 & 307,294 & 92,795 & 62,786 & 30,009 & $8 \%$ & 580,147 & 277,285 & 637,724 & $1.84 \%$ \\
\hline Mississippi & South & 372,618 & 177,813 & 51,369 & 34,775 & 16,594 & $22 \%$ & 337,843 & 161,219 & 384,213 & $1.11 \%$ \\
\hline Missouri & Midwest & 706,486 & 329,965 & 122,387 & 83,424 & 38,963 & $18 \%$ & 623,062 & 291,002 & 695,089 & $2.00 \%$ \\
\hline Montana & West & 111,919 & 58,840 & 8,711 & 5,709 & 3,002 & $22 \%$ & 106,210 & 55,838 & 126,080 & $0.36 \%$ \\
\hline Nebraska & Midwest & 220,531 & 108,467 & 42,141 & 28,248 & 13,893 & $12 \%$ & 192,283 & 94,574 & 215,980 & $0.62 \%$ \\
\hline Nevada & West & 246,987 & 101,505 & 13,926 & 9,870 & 4,056 & $17 \%$ & 237,117 & 97,449 & 250,087 & $0.72 \%$ \\
\hline New Hampshire & Northeast & 159,092 & 71,429 & 23,383 & 16,138 & 7,245 & $8 \%$ & 142,954 & 64,184 & 152,993 & $0.44 \%$ \\
\hline New Jersey & Northeast & $1,037,472$ & 422,469 & 198,631 & 141,152 & 57,479 & $10 \%$ & 896,320 & 364,990 & 925,577 & $2.67 \%$ \\
\hline New Mexico & West & 248,616 & 115,596 & 23,055 & 15,738 & 7,317 & $30 \%$ & 232,878 & 108,279 & 266,420 & $0.77 \%$ \\
\hline New York & Northeast & 2,288,991 & 937,738 & 475,942 & 337,626 & 138,316 & $22 \%$ & $1,951,365$ & 799,422 & $2,077,619$ & $5.99 \%$ \\
\hline North Carolina & South & 988,432 & 418,288 & 96,262 & 67,639 & 28,623 & $19 \%$ & 920,793 & 389,665 & 985,715 & $2.84 \%$ \\
\hline North Dakota & Midwest & 79,220 & 41,574 & 7,148 & 4,688 & 2,460 & $19 \%$ & 74,532 & 39,114 & 87,857 & $0.25 \%$ \\
\hline Ohio & Midwest & $1,439,754$ & 664,105 & 254,494 & 174,160 & 80,334 & $18 \%$ & $1,265,594$ & 583,771 & $1,402,225$ & $4.04 \%$ \\
\hline Oklahoma & South & 436,363 & 213,082 & 31,276 & 21,014 & 10,262 & $17 \%$ & 415,349 & 202,820 & 470,902 & $1.36 \%$ \\
\hline Oregon & West & 412,997 & 194,903 & 45,352 & 30,811 & 14,541 & $17 \%$ & 382,186 & 180,362 & 427,085 & $1.23 \%$ \\
\hline Pennsylvania & Northeast & $1,471,530$ & 668,898 & 339,484 & 233,393 & 106,091 & $13 \%$ & $1,238,137$ & 562,807 & $1,347,471$ & $3.88 \%$ \\
\hline Rhode Island & Northeast & 126,035 & 53,108 & 24,738 & 17,404 & 7,334 & $14 \%$ & 108,631 & 45,774 & 114,919 & $0.33 \%$ \\
\hline South Carolina & South & 484,743 & 217,725 & 55,612 & 38,375 & 17,237 & $18 \%$ & 446,368 & 200,488 & 488,797 & $1.41 \%$ \\
\hline South Dakota & Midwest & 98,587 & 49,664 & 9,364 & 6,227 & 3,137 & $7 \%$ & 92,360 & 46,527 & 103,803 & $0.30 \%$ \\
\hline Tennessee & South & 672,119 & 301,967 & 93,680 & 64,639 & 29,041 & $17 \%$ & 607,480 & 272,926 & 665,142 & $1.92 \%$ \\
\hline Texas & South & $2,828,626$ & $1,251,033$ & 227,645 & 157,837 & 69,808 & $22 \%$ & $2,670,789$ & $1,181,225$ & $2,937,498$ & $8.47 \%$ \\
\hline Utah & West & 336,843 & 160,082 & 12,614 & 8,550 & 4,064 & $7 \%$ & 328,293 & 156,018 & 359,260 & $1.04 \%$ \\
\hline Vermont & Northeast & 72,017 & 35,293 & 12,170 & $\begin{array}{ll}8,167 \\
\end{array}$ & 4,003 & $13 \%$ & 63,850 & 31,290 & 71,775 & $0.21 \%$ \\
\hline Virginia & South & 844,871 & 369,301 & 100,171 & 69,703 & 30,468 & $11 \%$ & 775,168 & 338,833 & 825,841 & $2.38 \%$ \\
\hline Washington & West & 750,722 & 345,682 & 76,885 & 52,644 & 24,241 & $11 \%$ & 698,078 & 321,441 & 760,091 & $2.19 \%$ \\
\hline West Virginia & South & 201,939 & 100,784 & 15,895 & 10,603 & 5,292 & $23 \%$ & 191,336 & 95,492 & 222,175 & $0.64 \%$ \\
\hline Wisconsin & Midwest & 683,234 & 333,212 & 139,455 & 93,739 & 45,716 & $9 \%$ & 589,495 & 287,496 & 656,101 & $1.89 \%$ \\
\hline Wyoming & West & 62,196 & 34,234 & 2,221 & 1,433 & 788 & $15 \%$ & 60,763 & 33,446 & 72,490 & $0.21 \%$ \\
\hline $\begin{array}{l}\text { Totals } \\
\end{array}$ & & 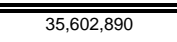 & $\overline{15,654,403}$ & $\overline{5,162,684}$ & 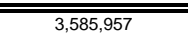 & $1,1,576,727$ & $\overline{17 \%}$ & $\begin{array}{l}32,016,933 \\
\end{array}$ & $\overline{c 14,077,676}$ & 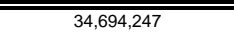 & $\overline{100.00 \%}$ \\
\hline
\end{tabular}


1999 Estimated College Population 1999 Workload Measure: Higher 1999 Population 1999 Population 1999 Population 1999 Population (Weighted by National \% Enrolled Education: Share of Estimated

\begin{tabular}{|c|c|c|c|c|c|c|c|}
\hline State & 1999 State Population & $\begin{array}{l}\text { Aged 14-17 } \\
\text { A }\end{array}$ & $\begin{array}{l}\text { Aged 18-24 } \\
\text { A }\end{array}$ & Aged 25-34 & Aged $35+$ & 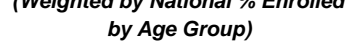 & College Population \\
\hline$\overline{\text { Alabama }}$ & $4,369,862$ & 244,544 & 439,534 & 614,609 & $2,249,542$ & 177,015 & $1.67 \%$ \\
\hline Alaska & 619,500 & 45,086 & 70,923 & 72,283 & 279,469 & 26,486 & $0.25 \%$ \\
\hline Arizona & $4,778,332$ & 280,312 & 459,678 & 628,940 & $2,355,150$ & 184,765 & $1.74 \%$ \\
\hline Arkansas & $2,551,373$ & 154,466 & 251,473 & 327,575 & $1,312,101$ & 100,545 & $0.95 \%$ \\
\hline California & $33,145,121$ & $1,814,758$ & $3,318,684$ & $5,114,990$ & $15,788,024$ & $1,346,593$ & $12.68 \%$ \\
\hline Colorado & $4,056,133$ & 245,902 & 392,703 & 523,975 & $2,073,945$ & 157,914 & $1.49 \%$ \\
\hline Connecticut & $3,282,031$ & 177,751 & 255,714 & 446,479 & $1,751,578$ & 112,163 & $1.06 \%$ \\
\hline Delaware & 753,538 & 39,989 & 69,255 & 113,221 & 388,612 & 29,040 & $0.27 \%$ \\
\hline D.C. & 519,000 & 17,304 & 45,671 & 95,007 & 283,032 & 20,296 & $0.19 \%$ \\
\hline Florida & $15,111,244$ & 783,677 & $1,235,957$ & $1,881,169$ & $8,424,240$ & 527,629 & $4.97 \%$ \\
\hline$\overline{\text { Georgia }}$ & $7,788,240$ & 447,609 & 773,918 & $1,205,249$ & $3,752,188$ & 315,613 & $2.97 \%$ \\
\hline Hawaii & $1,185,497$ & 61,690 & 119,733 & 146,817 & 629,607 & 47,353 & $0.45 \%$ \\
\hline Idaho & $1,251,700$ & 86,120 & 143,975 & 154,208 & 603,053 & 54,429 & $0.51 \%$ \\
\hline Illinois & $12,128,370$ & 691,806 & $1,143,197$ & $1,701,968$ & $6,101,867$ & 468,534 & $4.41 \%$ \\
\hline Indiana & $5,942,901$ & 351,397 & 576,310 & 823,952 & $3,013,648$ & 233,962 & $2.20 \%$ \\
\hline lowa & $2,869,413$ & 178,326 & 282,178 & 356,641 & $1,510,909$ & 112,751 & $1.06 \%$ \\
\hline Kansas & $2,654,052$ & 167,958 & 271,382 & 340,484 & $1,343,549$ & 107,156 & $1.01 \%$ \\
\hline Kentucky & $3,960,825$ & 227,336 & 404,621 & 542,574 & $2,048,102$ & 161,637 & $1.52 \%$ \\
\hline Louisiana & $4,372,035$ & 286,304 & 481,018 & 571,970 & $2,129,046$ & 185,713 & $1.75 \%$ \\
\hline Maine & $1,253,040$ & 74,023 & 110,630 & 166,472 & 685,499 & 46,478 & $0.44 \%$ \\
\hline Maryland & $5,171,634$ & 285,288 & 441,978 & 760,262 & $2,659,962$ & 189,308 & $1.78 \%$ \\
\hline Massachusetts & $6,175,169$ & 316,382 & 512,732 & 939,471 & $3,254,412$ & 223,925 & $2.11 \%$ \\
\hline Michigan & $9,863,775$ & 592,324 & 927,893 & $1,362,355$ & $5,012,388$ & 380,337 & $3.58 \%$ \\
\hline Minnesota & $4,775,508$ & 307,294 & 454,001 & 629,537 & $2,420,120$ & 184,186 & $1.73 \%$ \\
\hline Mississippi & $2,768,619$ & 177,813 & 302,471 & 378,055 & $1,335,227$ & 117,596 & $1.11 \%$ \\
\hline Missouri & $5,468,338$ & 329,965 & 520,487 & 721,307 & $2,827,052$ & 211,428 & $1.99 \%$ \\
\hline Montana & 882,779 & 58,840 & 89,389 & 94,112 & 475,459 & 34,810 & $0.33 \%$ \\
\hline Nebraska & $1,666,028$ & 108,467 & 170,141 & 206,359 & 845,728 & 66,911 & $0.63 \%$ \\
\hline Nevada & $1,809,253$ & 101,505 & 155,758 & 247,790 & 914,229 & 65,526 & $0.62 \%$ \\
\hline New Hampshire & $1,201,134$ & 71,429 & 98,125 & 178,006 & 620,567 & 42,867 & $0.40 \%$ \\
\hline New Jersey & $8,143,412$ & 422,469 & 672,511 & $1,106,507$ & $4,361,190$ & 288,732 & $2.72 \%$ \\
\hline New Mexico & $1,739,844$ & 115,596 & 176,216 & 210,923 & 857,093 & 69,010 & $0.65 \%$ \\
\hline New York & $18,196,601$ & 937,738 & $1,618,762$ & $2,622,029$ & $9,514,886$ & 682,066 & $6.42 \%$ \\
\hline North Carolina & $7,650,789$ & 418,288 & 709,470 & $1,111,053$ & $3,889,319$ & 294,318 & $2.77 \%$ \\
\hline North Dakota & 633,666 & 41,574 & 68,507 & $\begin{array}{l}77,782 \\
\end{array}$ & 327,285 & 26,529 & $0.25 \%$ \\
\hline Ohio & $11,256,654$ & 664,105 & $1,065,274$ & $1,531,688$ & $5,815,621$ & 435,550 & $4.10 \%$ \\
\hline Oklahoma & $3,358,044$ & 213,082 & 342,931 & 417,337 & $1,715,714$ & 134,983 & $1.27 \%$ \\
\hline Oregon & $3,316,154$ & 194,903 & 311,544 & 424,653 & $1,752,456$ & 126,802 & $1.19 \%$ \\
\hline Pennsylvania & $11,994,016$ & 668,898 & $1,025,209$ & $1,570,310$ & $6,545,977$ & 433,819 & $4.09 \%$ \\
\hline Rhode Island & 990,819 & 53,108 & 83,921 & 144,164 & 521,554 & 36,093 & $0.34 \%$ \\
\hline South Carolina & $3,885,736$ & 217,725 & 392,508 & 560,080 & $1,977,218$ & 158,281 & $1.49 \%$ \\
\hline South Dakota & 733,133 & 49,664 & 78,159 & 85,830 & 371,107 & 30,129 & $0.28 \%$ \\
\hline Tennessee & $5,483,535$ & 301,967 & 519,799 & 776,272 & $2,846,534$ & 213,750 & $2.01 \%$ \\
\hline Texas & $20,044,141$ & $1,251,033$ & $2,100,197$ & $2,771,877$ & $9,452,833$ & 825,586 & $7.77 \%$ \\
\hline Utah & $2,129,836$ & 160,082 & 300,984 & 292,796 & 828,690 & 107,725 & $1.01 \%$ \\
\hline Vermont & 593,740 & 35,293 & 53,195 & 83,144 & 318,055 & 22,374 & $0.21 \%$ \\
\hline Virginia & $6,872,912$ & 369,301 & 673,268 & $1,047,601$ & $3,487,233$ & 276,584 & $2.60 \%$ \\
\hline Washington & $5,756,361$ & 345,682 & 557,946 & 789,042 & $2,923,033$ & 226,210 & $2.13 \%$ \\
\hline West Virginia & $1,806,928$ & 100,784 & 179,418 & 226,085 & 997,944 & 71,900 & $0.68 \%$ \\
\hline Wisconsin & $5,250,446$ & 333,212 & 508,317 & 688,229 & $2,705,632$ & 205,265 & $1.93 \%$ \\
\hline Wyoming & 479,602 & 34,234 & 53,784 & 52,573 & 246,438 & 20,332 & $0.19 \%$ \\
\hline $\begin{array}{l}\text { Totals } \\
\end{array}$ & $272,690,813$ & $15,654,403$ & $26,011,449$ & $37,935,812$ & $138,544,117$ & $10,619,000$ & $100.00 \%$ \\
\hline
\end{tabular}


Public Welfare, 1999.

Percent of Persons in Poverty by Number of Persons in Poverty Workload Measure: Public Welfare: Share of Stat Census Region 1999 State Population State: 1998-1999 Average (calculated) Total Persons in Poverty

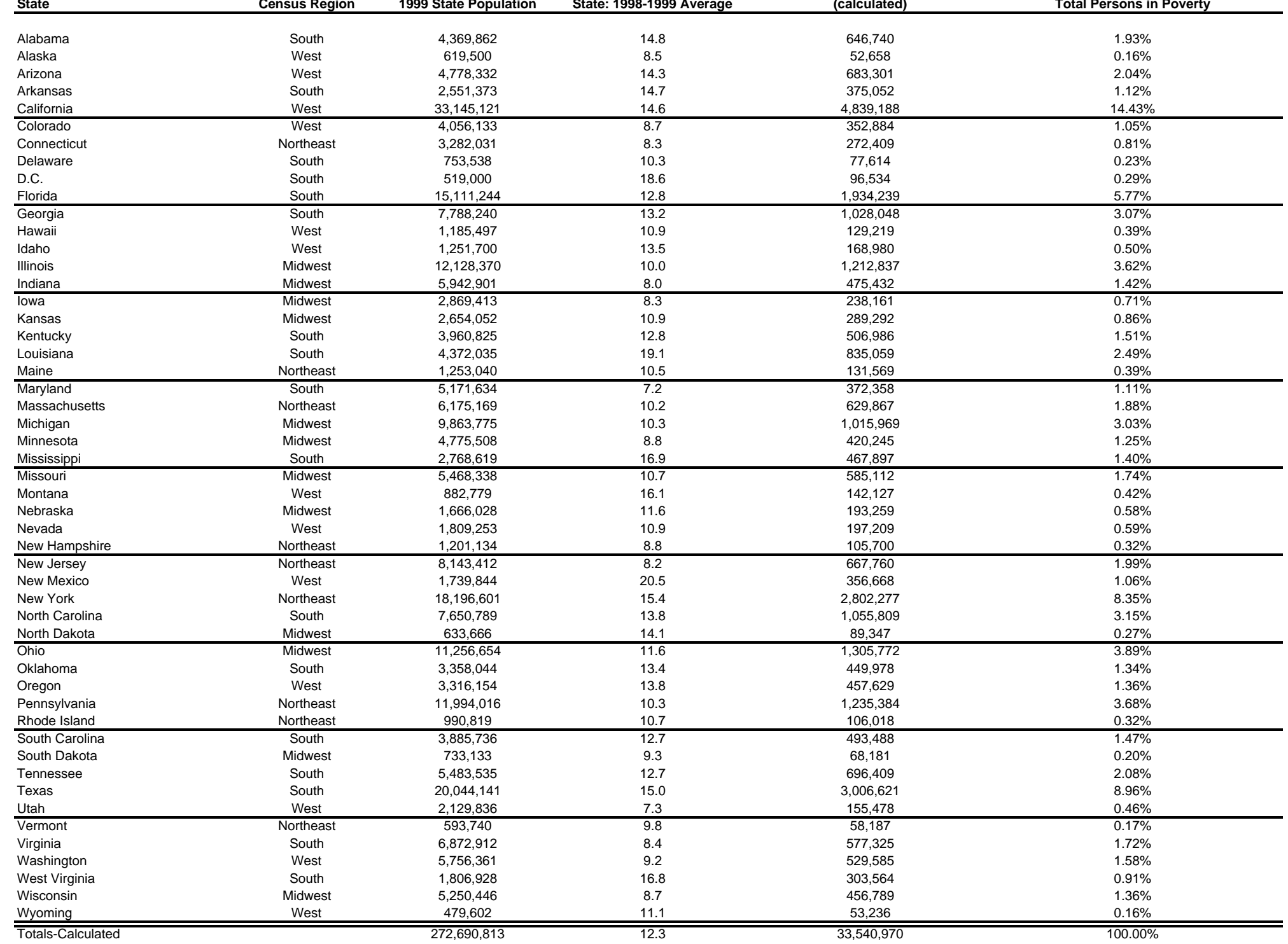


Health and Hospitals, 1999.

\begin{tabular}{|c|c|c|c|c|c|c|c|c|c|}
\hline State & $\begin{array}{l}\text { Census } \\
\text { Region } \\
\end{array}$ & $\begin{array}{c}\text { FY1999 Number } \\
\text { of Disabled } \\
\text { Workers }\end{array}$ & $\begin{array}{c}1999 \text { Share of Work - } \\
\text { Disabled Population } \\
\text { Aged 16-64 } \\
\end{array}$ & $\begin{array}{l}\text { Average Income } \\
\text { of Fourth and } \\
\text { Fifth: 1998-2000 }\end{array}$ & $\begin{array}{c}\text { Nation's 4th and 5th Income as a } \\
\text { Percent of the State 4th and 5th } \\
\text { Income }\end{array}$ & $\begin{array}{l}1999 \text { Share of 4th and 5th Income } \\
\text { Levels (share of population* national } \\
\text { income as a percent of state income) }\end{array}$ & $\begin{array}{l}1999 \text { State } \\
\text { Population }\end{array}$ & $\begin{array}{c}1999 \text { Share of } \\
\text { Total Population }\end{array}$ & $\begin{array}{l}1999 \text { Workload } \\
\text { Measure: Health } \\
\text { and Hospitals }\end{array}$ \\
\hline Alabama & South & 109,674 & $2.40 \%$ & 20,243 & $116.93 \%$ & $1.87 \%$ & $4,369,862$ & $1.60 \%$ & $1.96 \%$ \\
\hline Alaska & West & 6,311 & $0.14 \%$ & 29,289 & $80.81 \%$ & $0.18 \%$ & 619,500 & $0.23 \%$ & $0.18 \%$ \\
\hline Arizona & West & 77,424 & $1.69 \%$ & 21,189 & $111.71 \%$ & $1.96 \%$ & $4,778,332$ & $1.75 \%$ & $1.80 \%$ \\
\hline Arkansas & South & 72,365 & $1.58 \%$ & 19,148 & $123.61 \%$ & $1.16 \%$ & $2,551,373$ & $0.94 \%$ & $1.23 \%$ \\
\hline California & West & 404,574 & $8.85 \%$ & 22,434 & $105.51 \%$ & $12.82 \%$ & $33,145,121$ & $12.15 \%$ & $11.28 \%$ \\
\hline Colorado & West & 59,906 & $1.31 \%$ & 29,919 & $79.11 \%$ & $1.18 \%$ & $4,056,133$ & $1.49 \%$ & $1.33 \%$ \\
\hline Connecticut & Northeast & 50,108 & $1.10 \%$ & 31,309 & $75.60 \%$ & $0.91 \%$ & $3,282,031$ & $1.20 \%$ & $1.07 \%$ \\
\hline Delaware & South & 13,173 & $0.29 \%$ & 26,039 & $90.90 \%$ & $0.25 \%$ & 753,538 & $0.28 \%$ & $0.27 \%$ \\
\hline D.C. & South & 8,061 & $0.18 \%$ & 17,861 & $132.52 \%$ & $0.25 \%$ & 519,000 & $0.19 \%$ & $0.21 \%$ \\
\hline Florida & South & 281,198 & $6.15 \%$ & 22,071 & $107.24 \%$ & $5.94 \%$ & $15,111,244$ & $5.54 \%$ & $5.88 \%$ \\
\hline Georgia & South & 146,753 & $3.21 \%$ & 21,905 & $108.06 \%$ & $3.09 \%$ & $7,788,240$ & $2.86 \%$ & $3.05 \%$ \\
\hline Hawaii & West & 12,011 & $0.26 \%$ & 26,875 & $88.07 \%$ & $0.38 \%$ & $1,185,497$ & $0.43 \%$ & $0.36 \%$ \\
\hline Idaho & West & 18,649 & $0.41 \%$ & 21,491 & $110.14 \%$ & $0.51 \%$ & $1,251,700$ & $0.46 \%$ & $0.46 \%$ \\
\hline Illinois & Midwest & 167,881 & $3.67 \%$ & 26,510 & $89.29 \%$ & $3.97 \%$ & $12,128,370$ & $4.45 \%$ & $4.03 \%$ \\
\hline Indiana & Midwest & 103,530 & $2.27 \%$ & 27,129 & $87.25 \%$ & $1.90 \%$ & $5,942,901$ & $2.18 \%$ & $2.12 \%$ \\
\hline lowa & Midwest & 43,645 & $0.96 \%$ & 25,340 & $93.41 \%$ & $0.98 \%$ & $2,869,413$ & $1.05 \%$ & $1.00 \%$ \\
\hline Kansas & Midwest & 38,761 & $0.85 \%$ & 23,728 & $99.75 \%$ & $0.97 \%$ & $2,654,052$ & $0.97 \%$ & $0.93 \%$ \\
\hline Kentucky & South & 117,281 & $2.57 \%$ & 20,929 & $113.10 \%$ & $1.64 \%$ & $3,960,825$ & $1.45 \%$ & $1.89 \%$ \\
\hline Louisiana & South & 84,633 & $1.85 \%$ & 17,254 & $137.19 \%$ & $2.20 \%$ & $4,372,035$ & $1.60 \%$ & $1.88 \%$ \\
\hline Maine & Northeast & 31,925 & $0.70 \%$ & 24,408 & $96.97 \%$ & $0.45 \%$ & $1,253,040$ & $0.46 \%$ & $0.53 \%$ \\
\hline Maryland & South & 63,832 & $1.40 \%$ & 33,138 & $71.43 \%$ & $1.35 \%$ & $5,171,634$ & $1.90 \%$ & $1.55 \%$ \\
\hline Massachusetts & Northeast & 117,512 & $2.57 \%$ & 26,501 & $89.32 \%$ & $2.02 \%$ & $6,175,169$ & $2.26 \%$ & $2.29 \%$ \\
\hline Michigan & Midwest & 169,984 & $3.72 \%$ & 27,167 & $87.13 \%$ & $3.15 \%$ & $9,863,775$ & $3.62 \%$ & $3.50 \%$ \\
\hline Minnesota & Midwest & 62,606 & $1.37 \%$ & 31,636 & $74.82 \%$ & $1.31 \%$ & $4,775,508$ & $1.75 \%$ & $1.48 \%$ \\
\hline Mississippi & South & 78,742 & $1.72 \%$ & 18,499 & $127.95 \%$ & $1.30 \%$ & $2,768,619$ & $1.02 \%$ & $1.35 \%$ \\
\hline Missouri & Midwest & 111,681 & $2.44 \%$ & 25,649 & $92.28 \%$ & $1.85 \%$ & $5,468,338$ & $2.01 \%$ & $2.10 \%$ \\
\hline Montana & West & 16,147 & $0.35 \%$ & 18,818 & $125.78 \%$ & $0.41 \%$ & 882,779 & $0.32 \%$ & $0.36 \%$ \\
\hline Nebraska & Midwest & 23,206 & $0.51 \%$ & 24,591 & $96.25 \%$ & $0.59 \%$ & $1,666,028$ & $0.61 \%$ & $0.57 \%$ \\
\hline Nevada & West & 28,006 & $0.61 \%$ & 24,784 & $95.51 \%$ & $0.63 \%$ & $1,809,253$ & $0.66 \%$ & $0.64 \%$ \\
\hline New Hampshire & Northeast & 20,845 & $0.46 \%$ & 29,507 & $80.22 \%$ & $0.35 \%$ & $1,201,134$ & $0.44 \%$ & $0.42 \%$ \\
\hline New Jersey & Northeast & 116,683 & $2.55 \%$ & 30,588 & $77.38 \%$ & $2.31 \%$ & $8,143,412$ & $2.99 \%$ & $2.62 \%$ \\
\hline New Mexico & West & 29,656 & $0.65 \%$ & 17,720 & $133.58 \%$ & $0.85 \%$ & $1,739,844$ & $0.64 \%$ & $0.71 \%$ \\
\hline New York & Northeast & 321,930 & $7.04 \%$ & 22,034 & $107.42 \%$ & $7.17 \%$ & $18,196,601$ & $6.67 \%$ & $6.96 \%$ \\
\hline North Carolina & South & 179,808 & $3.93 \%$ & 21,466 & $110.27 \%$ & $3.09 \%$ & $7,650,789$ & $2.81 \%$ & $3.28 \%$ \\
\hline North Dakota & Midwest & 8,561 & $0.19 \%$ & 20,443 & $115.79 \%$ & $0.27 \%$ & 633,666 & $0.23 \%$ & $0.23 \%$ \\
\hline Ohio & Midwest & 188,806 & $4.13 \%$ & 24,528 & $96.50 \%$ & $3.98 \%$ & $11,256,654$ & $4.13 \%$ & $4.08 \%$ \\
\hline Oklahoma & South & 60,650 & $1.33 \%$ & 20,776 & $113.93 \%$ & $1.40 \%$ & $3,358,044$ & $1.23 \%$ & $1.32 \%$ \\
\hline Oregon & West & 50,895 & $1.11 \%$ & 23,110 & $102.42 \%$ & $1.25 \%$ & $3,316,154$ & $1.22 \%$ & $1.19 \%$ \\
\hline Pennsylvania & Northeast & 194,574 & $4.26 \%$ & 25,952 & $91.20 \%$ & $4.01 \%$ & $11,994,016$ & $4.40 \%$ & $4.22 \%$ \\
\hline Rhode Island & Northeast & 21,371 & $0.47 \%$ & 27,469 & $86.17 \%$ & $0.31 \%$ & 990,819 & $0.36 \%$ & $0.38 \%$ \\
\hline South Carolina & South & 93,889 & $2.05 \%$ & 22,741 & $104.08 \%$ & $1.48 \%$ & $3,885,736$ & $1.42 \%$ & $1.65 \%$ \\
\hline South Dakota & Midwest & 11,478 & $0.25 \%$ & 25,177 & $94.01 \%$ & $0.25 \%$ & 733,133 & $0.27 \%$ & $0.26 \%$ \\
\hline Tennessee & South & 133,005 & $2.91 \%$ & 20,686 & $114.43 \%$ & $2.30 \%$ & $5,483,535$ & $2.01 \%$ & $2.41 \%$ \\
\hline Texas & South & 244,911 & $5.36 \%$ & 20,559 & $115.13 \%$ & $8.46 \%$ & $20,044,141$ & $7.35 \%$ & $7.06 \%$ \\
\hline Utah & West & 20,549 & $0.45 \%$ & 28,339 & $83.52 \%$ & $0.65 \%$ & $2,129,836$ & $0.78 \%$ & $0.63 \%$ \\
\hline Vermont & Northeast & 11,547 & $0.25 \%$ & 24,170 & $97.93 \%$ & $0.21 \%$ & 593,740 & $0.22 \%$ & $0.23 \%$ \\
\hline Virginia & South & 116,839 & $2.56 \%$ & 28,296 & $83.65 \%$ & $2.11 \%$ & $6,872,912$ & $2.52 \%$ & $2.40 \%$ \\
\hline Washington & West & 82,608 & $1.81 \%$ & 27,576 & $85.83 \%$ & $1.81 \%$ & $5,756,361$ & $2.11 \%$ & $1.91 \%$ \\
\hline West Virginia & South & 55,900 & $1.22 \%$ & 17,941 & $131.93 \%$ & $0.87 \%$ & $1,806,928$ & $0.66 \%$ & $0.92 \%$ \\
\hline Wisconsin & Midwest & 78,249 & $1.71 \%$ & 27,810 & $85.11 \%$ & $1.64 \%$ & $5,250,446$ & $1.93 \%$ & $1.76 \%$ \\
\hline Wyoming & West & 7,456 & $0.16 \%$ & 22,882 & $103.44 \%$ & $0.18 \%$ & 479,602 & $0.18 \%$ & $0.17 \%$ \\
\hline United States Total & & $4, \overline{4,569,789}$ & $\overline{100.00 \%}$ & $\overline{23,670}$ & $\overline{100.00 \%}$ & $\overline{100.00 \%}$ & $\overline{272,690,813}$ & $\overline{100.00 \%}$ & $\overline{100.00 \%}$ \\
\hline
\end{tabular}


Police and Corrections, 1999

\begin{tabular}{|c|c|c|c|c|c|c|c|c|}
\hline State & $\begin{array}{l}\text { Census } \\
\text { Region } \\
\end{array}$ & $\begin{array}{c}\text { Murders and non- } \\
\text { negligent } \\
\text { manslaughter in } 1999\end{array}$ & $\begin{array}{c}1999 \text { Share of } \\
\text { Murders }\end{array}$ & $\begin{array}{c}1999 \text { State Population Aged } \\
18-24\end{array}$ & $\begin{array}{c}1999 \text { Share of Population } \\
\text { Aged 18-24 }\end{array}$ & $\begin{array}{l}1999 \text { Total } \\
\text { Population }\end{array}$ & $\begin{array}{c}1999 \text { Share of Total } \\
\text { Population }\end{array}$ & $\begin{array}{c}\text { Workload Measure: Police } \\
\text { and Corrections }\end{array}$ \\
\hline Alabama & South & 345 & $2.22 \%$ & 439,534 & $1.69 \%$ & $4,369,862$ & $1.60 \%$ & $1.84 \%$ \\
\hline Alaska & West & 53 & $0.34 \%$ & 70,923 & $0.27 \%$ & 619,500 & $0.23 \%$ & $0.28 \%$ \\
\hline Arizona & West & 384 & $2.47 \%$ & 459,678 & $1.77 \%$ & $4,778,332$ & $1.75 \%$ & $2.00 \%$ \\
\hline Arkansas & South & 143 & $0.92 \%$ & 251,473 & $0.97 \%$ & $2,551,373$ & $0.94 \%$ & $0.94 \%$ \\
\hline California & West & 2,005 & $12.91 \%$ & $3,318,684$ & $12.76 \%$ & $33,145,121$ & $12.15 \%$ & $12.61 \%$ \\
\hline Colorado & West & 185 & $1.19 \%$ & 392,703 & $1.51 \%$ & $4,056,133$ & $1.49 \%$ & $1.40 \%$ \\
\hline Connecticut & Northeast & 107 & $0.69 \%$ & 255,714 & $0.98 \%$ & $3,282,031$ & $1.20 \%$ & $0.96 \%$ \\
\hline Delaware & South & 24 & $0.15 \%$ & 69,255 & $0.27 \%$ & 753,538 & $0.28 \%$ & $0.23 \%$ \\
\hline D.C. & South & 241 & $1.55 \%$ & 45,671 & $0.18 \%$ & 519,000 & $0.19 \%$ & $0.64 \%$ \\
\hline Florida & South & 859 & $5.53 \%$ & $1,235,957$ & $4.75 \%$ & $15,111,244$ & $5.54 \%$ & $5.27 \%$ \\
\hline Georgia & South & 583 & $3.75 \%$ & 773,918 & $2.98 \%$ & $7,788,240$ & $2.86 \%$ & $3.19 \%$ \\
\hline Hawaii & West & 44 & $0.28 \%$ & 119,733 & $0.46 \%$ & $1,185,497$ & $0.43 \%$ & $0.39 \%$ \\
\hline Idaho & West & 25 & $0.16 \%$ & 143,975 & $0.55 \%$ & $1,251,700$ & $0.46 \%$ & $0.39 \%$ \\
\hline Illinois & Midwest & 937 & $6.03 \%$ & $1,143,197$ & $4.39 \%$ & $12,128,370$ & $4.45 \%$ & $4.96 \%$ \\
\hline Indiana & Midwest & 391 & $2.52 \%$ & 576,310 & $2.22 \%$ & $5,942,901$ & $2.18 \%$ & $2.30 \%$ \\
\hline lowa & Midwest & 43 & $0.28 \%$ & 282,178 & $1.08 \%$ & $2,869,413$ & $1.05 \%$ & $0.80 \%$ \\
\hline Kansas & Midwest & 160 & $1.03 \%$ & 271,382 & $1.04 \%$ & $2,654,052$ & $0.97 \%$ & $1.02 \%$ \\
\hline Kentucky & South & 212 & $1.36 \%$ & 404,621 & $1.56 \%$ & $3,960,825$ & $1.45 \%$ & $1.46 \%$ \\
\hline Louisiana & South & 468 & $3.01 \%$ & 481,018 & $1.85 \%$ & $4,372,035$ & $1.60 \%$ & $2.16 \%$ \\
\hline Maine & Northeast & 27 & $0.17 \%$ & 110,630 & $0.43 \%$ & $1,253,040$ & $0.46 \%$ & $0.35 \%$ \\
\hline Maryland & South & 465 & $2.99 \%$ & 441,978 & $1.70 \%$ & $5,171,634$ & $1.90 \%$ & $2.20 \%$ \\
\hline Massachusetts & Northeast & 122 & $0.79 \%$ & 512,732 & $1.97 \%$ & $6,175,169$ & $2.26 \%$ & $1.67 \%$ \\
\hline Michigan & Midwest & 695 & $4.47 \%$ & 927,893 & $3.57 \%$ & $9,863,775$ & $3.62 \%$ & $3.89 \%$ \\
\hline Minnesota & Midwest & 134 & $0.86 \%$ & 454,001 & $1.75 \%$ & $4,775,508$ & $1.75 \%$ & $1.45 \%$ \\
\hline Mississippi & South & 213 & $1.37 \%$ & 302,471 & $1.16 \%$ & $2,768,619$ & $1.02 \%$ & $1.18 \%$ \\
\hline Missouri & Midwest & 359 & $2.31 \%$ & 520,487 & $2.00 \%$ & $5,468,338$ & $2.01 \%$ & $2.11 \%$ \\
\hline Montana & West & 23 & $0.15 \%$ & 89,389 & $0.34 \%$ & 882,779 & $0.32 \%$ & $0.27 \%$ \\
\hline Nebraska & Midwest & 60 & $0.39 \%$ & 170,141 & $0.65 \%$ & $1,666,028$ & $0.61 \%$ & $0.55 \%$ \\
\hline Nevada & West & 165 & $1.06 \%$ & 155,758 & $0.60 \%$ & $1,809,253$ & $0.66 \%$ & $0.77 \%$ \\
\hline New Hampshire & Northeast & 18 & $0.12 \%$ & 98,125 & $0.38 \%$ & $1,201,134$ & $0.44 \%$ & $0.31 \%$ \\
\hline New Jersey & Northeast & 287 & $1.85 \%$ & 672,511 & $2.59 \%$ & $8,143,412$ & $2.99 \%$ & $2.47 \%$ \\
\hline New Mexico & West & 170 & $1.09 \%$ & 176,216 & $0.68 \%$ & $1,739,844$ & $0.64 \%$ & $0.80 \%$ \\
\hline New York & Northeast & 903 & $5.81 \%$ & $1,618,762$ & $6.22 \%$ & $18,196,601$ & $6.67 \%$ & $6.24 \%$ \\
\hline North Carolina & South & 552 & $3.55 \%$ & 709,470 & $2.73 \%$ & $7,650,789$ & $2.81 \%$ & $3.03 \%$ \\
\hline North Dakota & Midwest & 10 & $0.06 \%$ & 68,507 & $0.26 \%$ & 633,666 & $0.23 \%$ & $0.19 \%$ \\
\hline Ohio & Midwest & 397 & $2.56 \%$ & $1,065,274$ & $4.10 \%$ & $11,256,654$ & $4.13 \%$ & $3.59 \%$ \\
\hline Oklahoma & South & 231 & $1.49 \%$ & 342,931 & $1.32 \%$ & $3,358,044$ & $1.23 \%$ & $1.35 \%$ \\
\hline Oregon & West & 88 & $0.57 \%$ & 311,544 & $1.20 \%$ & $3,316,154$ & $1.22 \%$ & $0.99 \%$ \\
\hline Pennsylvania & Northeast & 592 & $3.81 \%$ & $1,025,209$ & $3.94 \%$ & $11,994,016$ & $4.40 \%$ & $4.05 \%$ \\
\hline Rhode Island & Northeast & 36 & $0.23 \%$ & 83,921 & $0.32 \%$ & $\begin{array}{c}990,819 \\
\end{array}$ & $0.36 \%$ & $0.31 \%$ \\
\hline South Carolina & South & 258 & $1.66 \%$ & 392,508 & $1.51 \%$ & $3,885,736$ & $1.42 \%$ & $1.53 \%$ \\
\hline South Dakota & Midwest & 18 & $0.12 \%$ & 78,159 & $0.30 \%$ & 733,133 & $0.27 \%$ & $0.23 \%$ \\
\hline Tennessee & South & 391 & $2.52 \%$ & 519,799 & $2.00 \%$ & $5,483,535$ & $2.01 \%$ & $2.18 \%$ \\
\hline Texas & South & 1,217 & $7.83 \%$ & $2,100,197$ & $8.07 \%$ & $20,044,141$ & $7.35 \%$ & $7.75 \%$ \\
\hline Utah & West & 44 & $0.28 \%$ & 300,984 & $1.16 \%$ & $2,129,836$ & $0.78 \%$ & $0.74 \%$ \\
\hline Vermont & Northeast & 17 & $0.11 \%$ & 53,195 & $0.20 \%$ & 593,740 & $0.22 \%$ & $0.18 \%$ \\
\hline Virginia & South & 392 & $2.52 \%$ & 673,268 & $2.59 \%$ & $6,872,912$ & $2.52 \%$ & $2.54 \%$ \\
\hline Washington & West & 171 & $1.10 \%$ & 557,946 & $2.15 \%$ & $5,756,361$ & $2.11 \%$ & $1.79 \%$ \\
\hline West Virginia & South & 79 & $0.51 \%$ & 179,418 & $0.69 \%$ & $1,806,928$ & $0.66 \%$ & $0.62 \%$ \\
\hline Wisconsin & Midwest & 179 & $1.15 \%$ & 508,317 & $1.95 \%$ & $5,250,446$ & $1.93 \%$ & $1.68 \%$ \\
\hline Wyoming & West & 11 & $0.07 \%$ & 53,784 & $0.21 \%$ & 479,602 & $0.18 \%$ & $0.15 \%$ \\
\hline $\begin{array}{l}\text { Totals } \\
\end{array}$ & & 15,533 & $\overline{100.00 \%}$ & $\overline{26,011,449}$ & $\overline{~ 100.00 \%}$ & $\overline{272,690,813}$ & 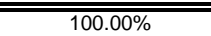 & $100.00 \%$ \\
\hline
\end{tabular}


Workload Measure: Highways: $\mathbf{8 2 . 5 \%}$ *

1999 Annual Vehicle- 1999 Share of 1999 Estimated 1999 Share of Lane Vehicle Miles Share $+(17.5 \%$ * Lane

\begin{tabular}{|c|c|c|c|c|c|c|c|}
\hline State & Census Region & 1999 State Population & Miles of Travel (millions) & Vehicle Miles & Lane Mileage & Miles & Miles Share) \\
\hline Alabama & South & $4,369,862$ & 56,165 & $2.087 \%$ & 195,170 & $2.387 \%$ & $2.14 \%$ \\
\hline Alaska & West & 619,500 & 4,545 & $0.169 \%$ & 25,674 & $0.314 \%$ & $0.19 \%$ \\
\hline Arizona & West & $4,778,332$ & 46,829 & $1.740 \%$ & 116,902 & $1.429 \%$ & $1.69 \%$ \\
\hline Arkansas & South & $2,551,373$ & 29,247 & $1.087 \%$ & 198,038 & $2.422 \%$ & $1.32 \%$ \\
\hline California & West & $33,145,121$ & 300,066 & $11.149 \%$ & 368,432 & $4.505 \%$ & $9.99 \%$ \\
\hline Colorado & West & $4,056,133$ & 40,732 & $1.513 \%$ & 176,435 & $2.157 \%$ & $1.63 \%$ \\
\hline Connecticut & Northeast & $3,282,031$ & 29,926 & $1.112 \%$ & 44,356 & $0.542 \%$ & $1.01 \%$ \\
\hline Delaware & South & 753,538 & 8,542 & $0.317 \%$ & 12,500 & $0.153 \%$ & $0.29 \%$ \\
\hline D.C. & South & 519,000 & 3,462 & $0.129 \%$ & 3,771 & $0.046 \%$ & $0.11 \%$ \\
\hline Florida & South & $15,111,244$ & 141,903 & $5.273 \%$ & 251,310 & $3.073 \%$ & $4.89 \%$ \\
\hline Georgia & South & $7,788,240$ & 98,859 & $3.673 \%$ & 239,290 & $2.926 \%$ & $3.54 \%$ \\
\hline Hawaii & West & $1,185,497$ & 8,116 & $0.302 \%$ & 9,202 & $0.113 \%$ & $0.27 \%$ \\
\hline Idaho & West & $1,251,700$ & 13,976 & $0.519 \%$ & 93,862 & $1.148 \%$ & $0.63 \%$ \\
\hline Illinois & Midwest & $12,128,370$ & 102,394 & $3.805 \%$ & 288,503 & $3.528 \%$ & $3.76 \%$ \\
\hline Indiana & Midwest & $5,942,901$ & 70,041 & $2.602 \%$ & 193,599 & $2.367 \%$ & $2.56 \%$ \\
\hline lowa & Midwest & $2,869,413$ & 29,138 & $1.083 \%$ & 231,448 & $2.830 \%$ & $1.39 \%$ \\
\hline Kansas & Midwest & $2,654,052$ & 27,699 & $1.029 \%$ & 272,743 & $3.335 \%$ & $1.43 \%$ \\
\hline Kentucky & South & $3,960,825$ & 47,816 & $1.777 \%$ & 153,819 & $1.881 \%$ & $1.79 \%$ \\
\hline Louisiana & South & $4,372,035$ & 41,205 & $1.531 \%$ & 127,763 & $1.562 \%$ & $1.54 \%$ \\
\hline Maine & Northeast & $1,253,040$ & 14,143 & $0.526 \%$ & 46,332 & $0.567 \%$ & $0.53 \%$ \\
\hline Maryland & South & $5,171,634$ & 49,126 & $1.825 \%$ & 66,630 & $0.815 \%$ & $1.65 \%$ \\
\hline Massachusetts & Northeast & $6,175,169$ & 51,820 & $1.925 \%$ & 74,424 & $0.910 \%$ & $1.75 \%$ \\
\hline Michigan & Midwest & $9,863,775$ & 95,644 & $3.554 \%$ & 255,589 & $3.125 \%$ & $3.48 \%$ \\
\hline Minnesota & Midwest & $4,775,508$ & 51,410 & $1.910 \%$ & 270,768 & $3.311 \%$ & $2.16 \%$ \\
\hline Mississippi & South & $2,768,619$ & 34,880 & $1.296 \%$ & 151,304 & $1.850 \%$ & $1.39 \%$ \\
\hline Missouri & Midwest & $5,468,338$ & 66,735 & $2.480 \%$ & 250,792 & $3.067 \%$ & $2.58 \%$ \\
\hline Montana & West & 882,779 & 9,835 & $0.365 \%$ & 142,177 & $1.739 \%$ & $0.61 \%$ \\
\hline Nebraska & Midwest & $1,666,028$ & 18,011 & $0.669 \%$ & 188,235 & $2.302 \%$ & $0.95 \%$ \\
\hline Nevada & West & $1,809,253$ & 17,391 & $0.646 \%$ & 75,035 & $0.918 \%$ & $0.69 \%$ \\
\hline New Hampshire & Northeast & $1,201,134$ & 11,894 & $0.442 \%$ & 31,264 & $0.382 \%$ & $0.43 \%$ \\
\hline New Jersey & Northeast & $8,143,412$ & 65,540 & $2.435 \%$ & 77,764 & $0.951 \%$ & $2.18 \%$ \\
\hline New Mexico & West & $1,739,844$ & 22,362 & $0.831 \%$ & 124,806 & $1.526 \%$ & $0.95 \%$ \\
\hline New York & Northeast & $18,196,601$ & 126,491 & $4.700 \%$ & 238,770 & $2.920 \%$ & $4.39 \%$ \\
\hline North Carolina & South & $7,650,789$ & 87,759 & $3.261 \%$ & 208,099 & $2.545 \%$ & $3.14 \%$ \\
\hline North Dakota & Midwest & 633,666 & 7,262 & $0.270 \%$ & 175,357 & $2.144 \%$ & $0.60 \%$ \\
\hline Ohio & Midwest & $11,256,654$ & 105,487 & $3.920 \%$ & 243,967 & $2.983 \%$ & $3.76 \%$ \\
\hline Oklahoma & South & $3,358,044$ & 42,569 & $1.582 \%$ & 232,310 & $2.841 \%$ & $1.80 \%$ \\
\hline Oregon & West & $3,316,154$ & 34,680 & $1.289 \%$ & 137,403 & $1.680 \%$ & $1.36 \%$ \\
\hline Pennsylvania & Northeast & $11,994,016$ & 102,014 & $3.790 \%$ & 248,692 & $3.041 \%$ & $3.66 \%$ \\
\hline Rhode Island & Northeast & $\begin{array}{c}990,819 \\
\end{array}$ & $\begin{array}{l}8,283 \\
8\end{array}$ & $0.308 \%$ & 12,813 & $0.157 \%$ & $0.28 \%$ \\
\hline South Carolina & South & $3,885,736$ & 44,146 & $1.640 \%$ & 136,006 & $1.663 \%$ & $1.64 \%$ \\
\hline South Dakota & Midwest & 733,133 & 8,244 & $0.306 \%$ & 168,940 & $2.066 \%$ & $0.61 \%$ \\
\hline Tennessee & South & $5,483,535$ & 64,755 & $2.406 \%$ & 183,273 & $2.241 \%$ & $2.38 \%$ \\
\hline Texas & South & $20,044,141$ & 210,874 & $7.835 \%$ & 637,812 & $7.799 \%$ & $7.83 \%$ \\
\hline Utah & West & $2,129,836$ & 22,044 & $0.819 \%$ & 86,637 & $1.059 \%$ & $0.86 \%$ \\
\hline Vermont & Northeast & 593,740 & 6,867 & $0.255 \%$ & 29,345 & $0.359 \%$ & $0.27 \%$ \\
\hline Virginia & South & $6,872,912$ & 73,904 & $2.746 \%$ & 152,249 & $1.862 \%$ & $2.59 \%$ \\
\hline Washington & West & $5,756,361$ & 52,714 & $1.959 \%$ & 167,513 & $2.048 \%$ & $1.97 \%$ \\
\hline West Virginia & South & $1,806,928$ & 19,033 & $0.707 \%$ & 74,772 & $0.914 \%$ & $0.74 \%$ \\
\hline Wisconsin & Midwest & $5,250,446$ & 56,960 & $2.116 \%$ & 230,397 & $2.817 \%$ & $2.24 \%$ \\
\hline Wyoming & West & 479,602 & 7,797 & $0.290 \%$ & 55,686 & $0.681 \%$ & $0.36 \%$ \\
\hline
\end{tabular}


Median Annual Earnings of All Workers

45-54 Years Old who were Year Round Full Time Workers in 1999,

Weighted by 1999 Weights of Educational Attainment

Actual State Median 45-54

Some College, No

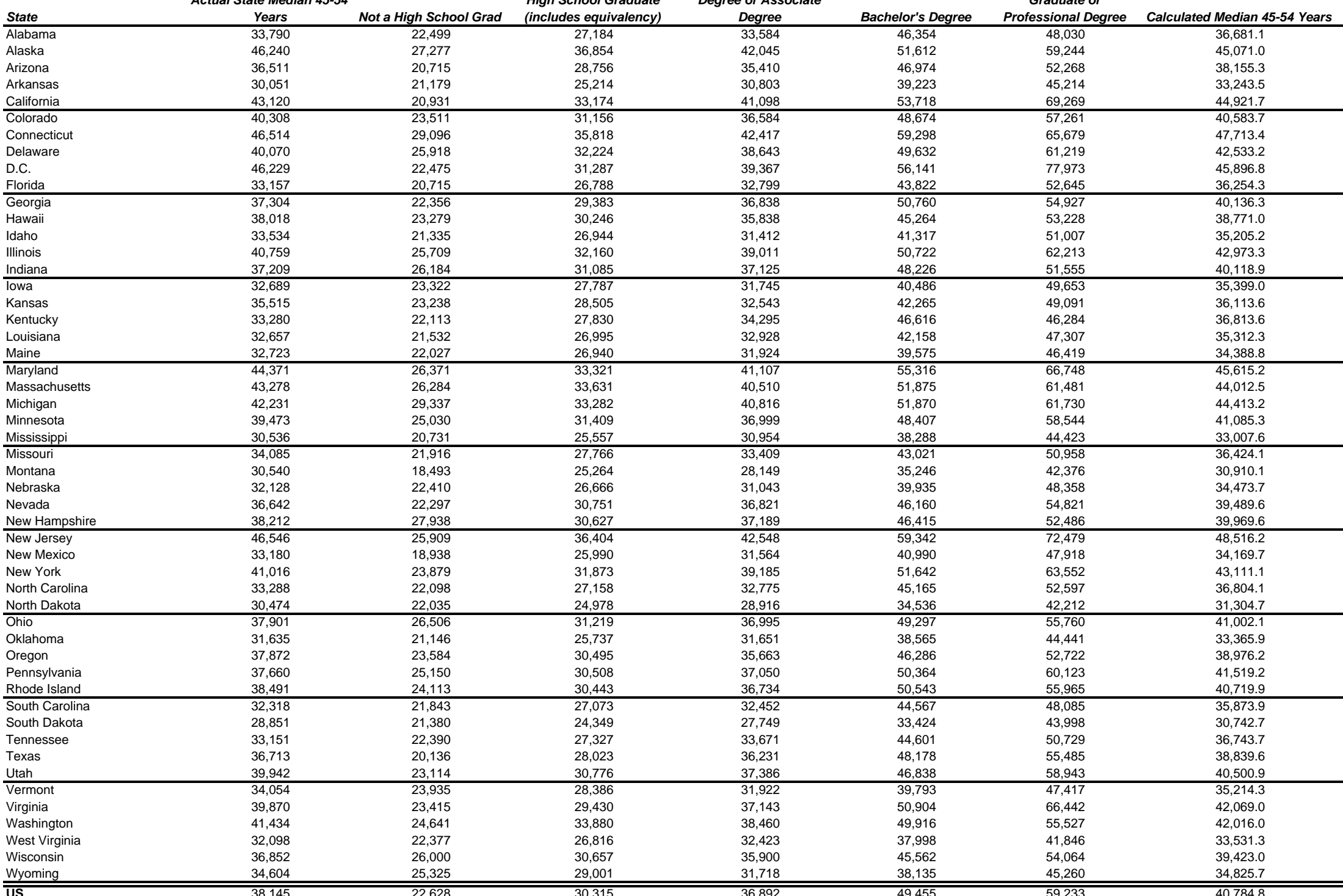


Indicies of Median Annual Earnings: Year round, full time workers, ager 45-54

\begin{tabular}{|c|c|c|c|c|c|c|c|}
\hline State & $\begin{array}{c}\text { Actual State } \\
\text { Median 45-54 } \\
\text { Years } \\
\end{array}$ & $\begin{array}{c}\text { Not a High } \\
\text { School Grad } \\
\end{array}$ & $\begin{array}{c}\text { High School } \\
\text { Graduate } \\
\text { (includes } \\
\text { equivalency) }\end{array}$ & $\begin{array}{c}\text { Some College, } \\
\text { No Degree or } \\
\text { Associate } \\
\text { Degree }\end{array}$ & $\begin{array}{c}\begin{array}{c}\text { Bachelor's } \\
\text { Degree }\end{array} \\
\end{array}$ & $\begin{array}{c}\text { Graduate or } \\
\text { Professional } \\
\text { Degree } \\
\end{array}$ & $\begin{array}{c}\text { Calculated } \\
\text { Median 45-54 } \\
\text { Years } \\
\end{array}$ \\
\hline Alabama & 89 & 99 & 90 & 91 & 94 & 81 & 90 \\
\hline Alaska & 121 & 121 & 122 & 114 & 104 & 100 & 111 \\
\hline Arizona & 96 & 92 & 95 & 96 & 95 & 88 & 94 \\
\hline Arkansas & 79 & 94 & 83 & 83 & 79 & 76 & 82 \\
\hline California & 113 & 93 & 109 & 111 & 109 & 117 & 110 \\
\hline Colorado & 106 & 104 & 103 & 99 & 98 & 97 & 100 \\
\hline Connecticut & 122 & 129 & 118 & 115 & 120 & 111 & 117 \\
\hline Delaware & 105 & 115 & 106 & 105 & 100 & 103 & 104 \\
\hline D.C. & 121 & 99 & 103 & 107 & 114 & 132 & 113 \\
\hline Florida & 87 & 92 & 88 & 89 & 89 & 89 & 89 \\
\hline Georgia & 98 & 99 & 97 & 100 & 103 & 93 & 98 \\
\hline Hawaii & 100 & 103 & 100 & 97 & 92 & 90 & 95 \\
\hline Idaho & 88 & 94 & 89 & 85 & 84 & 86 & 86 \\
\hline Illinois & 107 & 114 & 106 & 106 & 103 & 105 & 105 \\
\hline Indiana & 98 & 116 & 103 & 101 & 98 & 87 & 98 \\
\hline lowa & 86 & 103 & 92 & 86 & 82 & 84 & 87 \\
\hline Kansas & 93 & 103 & 94 & 88 & 85 & 83 & 89 \\
\hline Kentucky & 87 & 98 & 92 & 93 & 94 & 78 & 90 \\
\hline Louisiana & 86 & 95 & 89 & 89 & 85 & 80 & 87 \\
\hline Maine & 86 & 97 & 89 & 87 & 80 & 78 & 84 \\
\hline Maryland & 116 & 117 & 110 & 111 & 112 & 113 & 112 \\
\hline Massachusetts & 113 & 116 & 111 & 110 & 105 & 104 & 108 \\
\hline Michigan & 111 & 130 & 110 & 111 & 105 & 104 & 109 \\
\hline Minnesota & 103 & 111 & 104 & 100 & 98 & 99 & 101 \\
\hline Mississippi & 80 & 92 & 84 & 84 & 77 & 75 & 81 \\
\hline Missouri & 89 & 97 & 92 & 91 & 87 & 86 & 89 \\
\hline Montana & 80 & 82 & 83 & 76 & 71 & 72 & 76 \\
\hline Nebraska & 84 & 99 & 88 & 84 & 81 & 82 & 85 \\
\hline Nevada & 96 & 99 & 101 & 100 & 93 & 93 & 97 \\
\hline New Hampshire & 100 & 123 & 101 & 101 & 94 & 89 & 98 \\
\hline New Jersey & 122 & 114 & 120 & 115 & 120 & 122 & 119 \\
\hline New Mexico & 87 & 84 & 86 & 86 & 83 & 81 & 84 \\
\hline New York & 108 & 106 & 105 & 106 & 104 & 107 & 106 \\
\hline North Carolina & 87 & 98 & 90 & 89 & 91 & 89 & 90 \\
\hline North Dakota & 80 & 97 & 82 & 78 & 70 & 71 & 77 \\
\hline Ohio & 99 & 117 & 103 & 100 & 100 & 94 & 101 \\
\hline Oklahoma & 83 & 93 & 85 & 86 & 78 & 75 & 82 \\
\hline Oregon & 99 & 104 & 101 & 97 & 94 & 89 & 96 \\
\hline Pennsylvania & 99 & 111 & 101 & 100 & 102 & 102 & 102 \\
\hline Rhode Island & 101 & 107 & 100 & 100 & 102 & 94 & 100 \\
\hline South Carolina & 85 & 97 & 89 & 88 & 90 & 81 & 88 \\
\hline South Dakota & 76 & 94 & 80 & 75 & 68 & 74 & 75 \\
\hline Tennessee & 87 & 99 & 90 & 91 & 90 & 86 & 90 \\
\hline Texas & 96 & 89 & 92 & 98 & 97 & 94 & 95 \\
\hline Utah & 105 & 102 & 102 & 101 & 95 & 100 & 99 \\
\hline Vermont & 89 & 106 & 94 & 87 & 80 & 80 & 86 \\
\hline Virginia & 105 & 103 & 97 & 101 & 103 & 112 & 103 \\
\hline Washington & 109 & 109 & 112 & 104 & 101 & 94 & 103 \\
\hline West Virginia & 84 & 99 & 88 & 88 & 77 & 71 & 82 \\
\hline Wisconsin & 97 & 115 & 101 & 97 & 92 & 91 & 97 \\
\hline Wyoming & 91 & 112 & 96 & 86 & 77 & 76 & 85 \\
\hline US & $\overline{100}$ & 100 & $\overline{100}$ & 100 & 100 & $\overline{100}$ & 100 \\
\hline
\end{tabular}


Number of Workers in Each Category by Educational Attainment 1999

$\begin{array}{ccccccc} & \text { Totals } & \begin{array}{c}\text { Not a High } \\ \text { School Grad }\end{array} & \begin{array}{c}\text { High School } \\ \text { Graduate (includes } \\ \text { equivalency) }\end{array} & \begin{array}{c}\text { Some College, } \\ \text { No Degree or } \\ \text { Associate Degree }\end{array} & \begin{array}{c}\text { Bachelor's } \\ \text { Degree }\end{array} & \begin{array}{c}\text { Graduate or } \\ \text { Professional } \\ \text { Degree }\end{array} \\ \begin{array}{l}\text { Percentage } \\ \text { Distribution }\end{array} & 152,921,810 & 19,543,685 & 44,071,390 & 48,530,160 & 26,875,435 & 13,901,140 \\ & 1.0000 & 0.1278 & 0.2882 & 0.3174 & 0.1757 & 0.0909\end{array}$


Input-Cost Indices for Major Functions, 1999.

\begin{tabular}{|c|c|c|c|c|c|c|c|c|c|c|c|}
\hline $\begin{array}{l}\text { Index } \\
\text { Compensation Costs as \% of }\end{array}$ & $\begin{array}{l}\text { Unit Labor } \\
\text { Cost Index }\end{array}$ & $\begin{array}{l}\text { Primary and } \\
\text { Secondary } \\
\text { Education }\end{array}$ & $\begin{array}{c}\text { Higher } \\
\text { Education }\end{array}$ & $\begin{array}{l}\text { Public } \\
\text { Welfare }\end{array}$ & $\begin{array}{l}\text { Health and } \\
\text { Hospitals }\end{array}$ & Highways & $\begin{array}{l}\text { Police and } \\
\text { Corrections }\end{array}$ & $\begin{array}{l}\text { Environment and } \\
\text { Housing }\end{array}$ & $\begin{array}{l}\text { Interest on } \\
\text { General debt }\end{array}$ & $\begin{array}{l}\text { Governmental } \\
\text { Administration }\end{array}$ & All Other \\
\hline Total Costs & & $67.3 \%$ & $65.4 \%$ & $9.5 \%$ & $50.8 \%$ & $26.9 \%$ & $80.6 \%$ & $29.3 \%$ & $0.0 \%$ & $65.1 \%$ & $44.2 \%$ \\
\hline Alabama & 89.9 & 93.2 & 93.4 & 99.0 & 94.9 & 97.3 & 91.9 & 97.1 & 100.0 & 93.4 & 95.6 \\
\hline Alaska & 110.5 & 107.1 & 106.9 & 101.0 & 105.3 & 102.8 & 108.5 & 103.1 & 100.0 & 106.8 & 104.6 \\
\hline Arizona & 93.6 & 95.7 & 95.8 & 99.4 & 96.7 & 98.3 & 94.8 & 98.1 & 100.0 & 95.8 & 97.2 \\
\hline Arkansas & 81.5 & 87.5 & 87.9 & 98.2 & 90.6 & 95.0 & 85.1 & 94.6 & 100.0 & 88.0 & 91.8 \\
\hline California & 110.1 & 106.8 & 106.6 & 101.0 & 105.1 & 102.7 & 108.2 & 103.0 & 100.0 & 106.6 & 104.5 \\
\hline Colorado & 99.5 & 99.7 & 99.7 & 100.0 & 99.7 & 99.9 & 99.6 & 99.9 & 100.0 & 99.7 & 99.8 \\
\hline Connecticut & 117.0 & 111.4 & 111.1 & 101.6 & 108.6 & 104.6 & 113.7 & 105.0 & 100.0 & 111.1 & 107.5 \\
\hline Delaware & 104.3 & 102.9 & 102.8 & 100.4 & 102.2 & 101.2 & 103.5 & 101.3 & 100.0 & 102.8 & 101.9 \\
\hline District of Columbia & 112.5 & 108.4 & 108.2 & 101.2 & 106.4 & 103.4 & 110.1 & 103.7 & 100.0 & 108.2 & 105.5 \\
\hline $\begin{array}{l}\text { Florida } \\
\text { Finda }\end{array}$ & 88.9 & 92.5 & 92.7 & 98.9 & $\begin{array}{l}94.4 \\
94.4\end{array}$ & $\begin{array}{l}97.0 \\
97.0\end{array}$ & 91.0 & 96.7 & 100.0 & 92.8 & 95.1 \\
\hline Georgia & 98.4 & 98.9 & 99.0 & 99.8 & 99.2 & 99.6 & 98.7 & 99.5 & 100.0 & 99.0 & 99.3 \\
\hline Hawaii & 95.1 & 96.7 & 96.8 & 99.5 & 97.5 & 98.7 & 96.0 & 98.6 & 100.0 & 96.8 & 97.8 \\
\hline Idaho & 86.3 & 90.8 & 91.1 & 98.7 & 93.1 & 96.3 & 89.0 & 96.0 & 100.0 & 91.1 & 94.0 \\
\hline Illinois & 105.4 & 103.6 & 103.5 & 100.5 & 102.7 & 101.4 & 104.3 & 101.6 & 100.0 & 103.5 & 102.4 \\
\hline Indiana & 98.4 & 98.9 & 98.9 & 99.8 & 99.2 & 99.6 & 98.7 & 99.5 & 100.0 & 98.9 & 99.3 \\
\hline lowa & 86.8 & 91.1 & 91.4 & 98.7 & 93.3 & 96.5 & 89.4 & 96.1 & 100.0 & 91.4 & 94.2 \\
\hline Kansas & 88.5 & 92.3 & 92.5 & 98.9 & 94.2 & 96.9 & 90.8 & 96.6 & 100.0 & 92.5 & 94.9 \\
\hline Kentucky & 90.3 & 93.4 & 93.6 & 99.1 & 95.1 & 97.4 & 92.1 & 97.1 & 100.0 & 93.7 & 95.7 \\
\hline Louisiana & 86.6 & 91.0 & 91.2 & 98.7 & 93.2 & 96.4 & 89.2 & 96.1 & 100.0 & 91.3 & 94.1 \\
\hline Maine & 84.3 & 89.4 & 89.7 & 98.5 & 92.0 & 95.8 & 87.4 & 95.4 & 100.0 & 89.8 & 93.1 \\
\hline Maryland & 111.8 & 108.0 & 107.7 & 101.1 & 106.0 & 103.2 & 109.6 & 103.5 & 100.0 & 107.7 & 105.2 \\
\hline Massachusetts & 107.9 & 105.3 & 105.2 & 100.8 & 104.0 & 102.1 & 106.4 & 102.3 & 100.0 & 105.2 & 103.5 \\
\hline Michigan & 108.9 & 106.0 & 105.8 & 100.8 & 104.5 & 102.4 & 107.2 & 102.6 & 100.0 & 105.8 & 103.9 \\
\hline Minnesota & 100.7 & 100.5 & 100.5 & 100.1 & 100.4 & 100.2 & 100.6 & 100.2 & 100.0 & 100.5 & 100.3 \\
\hline Mississippi & 80.9 & 87.2 & 87.5 & 98.2 & 90.3 & 94.9 & 84.6 & 94.4 & 100.0 & 87.6 & 91.6 \\
\hline Missouri & 89.3 & 92.8 & 93.0 & 99.0 & 94.6 & 97.1 & 91.4 & 96.9 & 100.0 & 93.0 & 95.3 \\
\hline Montana & 75.8 & 83.7 & 84.2 & 97.7 & 87.7 & 93.5 & 80.5 & 92.9 & 100.0 & 84.2 & 89.3 \\
\hline Nebraska & 84.5 & 89.6 & 89.9 & 98.5 & 92.1 & 95.8 & 87.5 & 95.5 & 100.0 & 89.9 & 93.2 \\
\hline Nevada & 96.8 & 97.9 & 97.9 & 99.7 & 98.4 & 99.1 & 97.4 & 99.1 & 100.0 & 97.9 & 98.6 \\
\hline New Hampshire & 98.0 & 98.7 & 98.7 & 99.8 & 99.0 & 99.5 & 98.4 & 99.4 & 100.0 & 98.7 & 99.1 \\
\hline New Jersey & 119.0 & 112.8 & 112.4 & 101.8 & 109.6 & 105.1 & 115.3 & 105.6 & 100.0 & 112.3 & 108.4 \\
\hline New Mexico & 83.8 & 89.1 & 89.4 & 98.5 & 91.8 & 95.6 & 86.9 & 95.2 & 100.0 & 89.4 & 92.8 \\
\hline New York & 105.7 & 103.8 & 103.7 & 100.5 & 102.9 & 101.5 & 104.6 & 101.7 & 100.0 & 103.7 & 102.5 \\
\hline North Carolina & 90.2 & 93.4 & 93.6 & 99.1 & 95.0 & 97.4 & 92.1 & 97.1 & 100.0 & 93.6 & 95.7 \\
\hline North Dakota & 76.8 & 84.3 & 84.8 & 97.8 & 88.2 & 93.8 & 81.3 & 93.2 & 100.0 & 84.9 & 89.7 \\
\hline Ohio & 100.5 & 100.4 & 100.3 & 100.1 & 100.3 & 100.1 & 100.4 & 100.2 & 100.0 & 100.3 & 100.2 \\
\hline Oklahoma & 81.8 & 87.7 & 88.1 & 98.3 & 90.8 & 95.1 & 85.3 & 94.7 & 100.0 & 88.2 & 92.0 \\
\hline Oregon & 95.6 & 97.0 & 97.1 & 99.6 & 97.7 & 98.8 & 96.4 & 98.7 & 100.0 & 97.1 & 98.0 \\
\hline Pennsylvania & 101.8 & 101.2 & 101.2 & 100.2 & 100.9 & 100.5 & 101.5 & 100.5 & 100.0 & 101.2 & 100.8 \\
\hline Rhode Island & 99.8 & 99.9 & 99.9 & 100.0 & 99.9 & 100.0 & 99.9 & 100.0 & 100.0 & 99.9 & 99.9 \\
\hline South Carolina & 88.0 & 91.9 & 92.1 & 98.9 & 93.9 & 96.8 & 90.3 & 96.5 & 100.0 & 92.2 & 94.7 \\
\hline South Dakota & 75.4 & 83.4 & 83.9 & 97.7 & 87.5 & 93.4 & 80.1 & 92.8 & 100.0 & 84.0 & 89.1 \\
\hline Tennessee & 90.1 & 93.3 & 93.5 & 99.1 & 95.0 & 97.3 & 92.0 & 97.1 & 100.0 & 93.5 & 95.6 \\
\hline Texas & 95.2 & 96.8 & 96.9 & 99.5 & 97.6 & 98.7 & 96.2 & 98.6 & 100.0 & 96.9 & 97.9 \\
\hline Utah & 99.3 & 99.5 & 99.5 & 99.9 & 99.6 & 99.8 & 99.4 & 99.8 & 100.0 & 99.5 & 99.7 \\
\hline Vermont & 86.3 & 90.8 & 91.1 & 98.7 & 93.1 & 96.3 & 89.0 & 96.0 & 100.0 & 91.1 & 94.0 \\
\hline Virginia & 103.1 & 102.1 & 102.1 & 100.3 & 101.6 & 100.8 & 102.5 & 100.9 & 100.0 & 102.1 & 101.4 \\
\hline Washington & 103.0 & 102.0 & 102.0 & 100.3 & 101.5 & 100.8 & 102.4 & 100.9 & 100.0 & 102.0 & 101.3 \\
\hline West Virginia & 82.2 & 88.0 & 88.4 & 98.3 & 91.0 & 95.2 & 85.7 & 94.8 & 100.0 & 88.4 & 92.1 \\
\hline Wisconsin & 96.7 & 97.8 & 97.8 & 99.7 & 98.3 & 99.1 & 97.3 & 99.0 & 100.0 & 97.8 & 98.5 \\
\hline Wyoming & 85.4 & 90.2 & 90.4 & 98.6 & 92.6 & 96.1 & 88.2 & 95.7 & 100.0 & 90.5 & 93.5 \\
\hline $\begin{array}{l}\text { Totals } \\
\end{array}$ & "100.0 & 年100.0 & 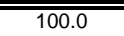 & 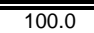 & 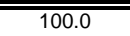 & 100.0 & "100.0 & 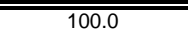 & 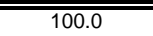 & 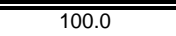 & 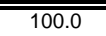 \\
\hline
\end{tabular}

